\title{
Signal transduction by the essential nucleotide second messenger cyclic di-AMP in Bacillus subtilis
}

\section{Dissertation}

for the award of the degree

"Doctor rerum naturalium"

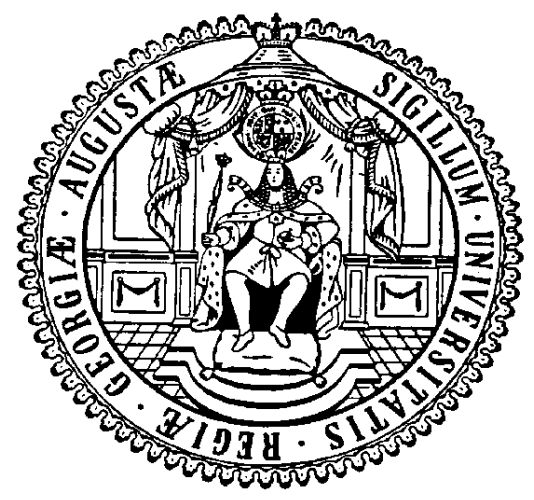

Division of Mathematics and Natural Sciences of the Georg-August-Universität Göttingen within the doctoral program "Microbiology \& Biochemistry" of the Georg-August-University School of Science (GAUSS)

submitted by

\section{Larissa Krüger}

from Kassel 



\section{Thesis Committee}

\section{Prof. Dr. Jörg Stülke (Supervisor and 1st Reviewer)}

Institute of Microbiology and Genetics, Department of General Microbiology, University of Göttingen

Prof. Dr. Ralf Ficner (2nd Reviewer)

Institute of Microbiology and Genetics, Department of Molecular Structural Biology, University of Göttingen

Prof Dr. Fabian M. Commichau

Institute for Biotechnology, Department of Synthetic Microbiology, Brandenburg University of Technology, Cottbus-Senftenberg

\section{Further Members of the Examination Board}

\section{Dr. Sarah Adio}

Department of Physical Biochemistry, Max Planck Institute for Biophysical Chemistry, Göttingen

\section{Prof. Dr. Stefanie Pöggeler}

Institute of Microbiology and Genetics, Department of Genetics of Eukaryotic Microorganisms, University of Göttingen

\section{Prof. Dr. Henning Urlaub}

Bioanalytical Mass Spectrometry, Max Planck Institute for Biophysical Chemistry, Göttingen

Date of oral examination: 11.12.2020 


\section{Statement of Authorship}

I hereby declare that the doctoral thesis entitled "Signal transduction by the essential nucleotide second messenger cyclic di-AMP in Bacillus subtilis" has been written independently and with no other sources and aids than quoted.

Larissa Krüger 
„Zwei Dinge sind zu unserer Arbeit nötig: Unermüdliche Ausdauer und die Bereitschaft, etwas, in das man viel Zeit und Arbeit gesteckt hat, wieder wegzuwerfen."

- Albert Einstein - 


\section{Danksagung}

Lieber Jörg, ich danke dir für die tolle Betreuung und für jegliche Unterstützung, die du mir in den letzten Jahren entgegengebracht hast. Danke, dass du mir die Möglichkeit gegegeben hast, meine Arbeit in deiner Abteilung anzufertigen und dass du mir dieses spannende Projekt anvertraut hast. Ich hatte immer das Gefühl, mit dir über alles reden zu können, sowohl wissenschaftlich als auch privat, und das schätze ich sehr. Deine Begeisterung für die Forschung ist wirklich toll und ich habe es immer genossen mit dir über neue Ideen zu diskutieren.

Liebe Christina, nur mit deiner Unterstützung ist diese Arbeit zu dem geworden, was sie ist. Du warst immer mit voller Energie dabei und deine positive Art hat mich oft vor dem Verzweifeln gerettet. Ich glaube es wäre mit niemanden anders möglich gewesen morgens Corona Proben anzulegen und anschließend im Labor gemeinsam nochmal so Gas zu geben, und das ist nur ein Beispiel von vielen. Ich glaube wir sind mit der Zeit zu einem super Team zusammengewachsen und es war für mich eine große Freude mit dir gemeinsam zu forschen. Ich bin dir sehr dankbar für die tolle Zusammenarbeit. An der tollen Atmosphäre im Labor hattest auch du Rica einen großen Anteil und ich bin sehr froh, dass du unser Labor mit deiner aufgeweckten und lieben Art bereichert hast. An dieser Stelle möchte ich auch Gabriele für ihre Unterstützung im Labor danken, ich hoffe du hast die Zeit genauso genossen wie wir. Mein Corona-bedingter Umzug in das "Nerd-Office“ von Tiago hat meine letzten Monate im Labor nicht nur musikalisch aufgewertet. Ich habe die Zeit wirklich genossen und ich danke dir sehr für deine endlose Hilfsbereitschaft und die viele Schokolade, die so manche Rückschläge erträglicher gemacht hat.

Des Weiteren danke ich meinen Studenten Mats Koschel, Iljana Gerlitzki, Simon Bolz, Robert Warneke, Lorenz Demann und Dennis Wicke für ihren Beitrag zu dieser Arbeit. Es hat mir immer Spaß gemacht mit euch zusammenzuarbeiten und zu sehen, dass die Begeisterung für die Wissenschaft eben doch ansteckend sein kann.

Prof. Dr. Fabian Commichau und Prof. Dr. Ralf Ficner danke ich, dass sie Teil meines Thesis Advisory Committee waren und für immer neue interessante Ideen. Dr. Sarah Adio, Prof. Dr. Stephanie Pöggeler, und Prof. Dr. Henning Urlaub danke ich für die Teilnahme an der Prüfungskommission.

Ein großer Dank geht auch an unsere vielen Kooperationspartner, die zu einem großen Teil zum Gelingen dieser Arbeit beigetragen haben. Allen voran Annette Garbe und Heike Bähre für die erstklassigen (und unzähligen) Nukleotidbestimmungen und dafür, dass ihr uns unsere Ungeduld nie übel genommen habt. Unseren Nachbarn aus der Strukturbiologie Jana Heidemann, Achim Dickmanns und Piotr Neumann danke ich für die tolle Zusammenarbeit, es hat immer Spaß gemacht gemeinsam mit Euch über DarB zu diskutieren. Ein großer Dank geht auch an Anja Poehlein und Rolf Daniel für die etlichen Genomsequenzierungen, die ihr für mich durchgeführt habt, und die wirklich sehr oft Licht ins 
Dunkel gebracht haben. Kerstin Schmitt und Oliver Valerius danke ich für die Durchführung der Proteomics und dafür, dass ihr immer ein offenes Ohr hattet. Inga Hähnelt gebührt Dank für die Kooperation zu KtrCD.

Nicht zu vergessen sind Anika und Cedric, von denen ich viel lernen konnte und mit denen ich seit meiner Bachelorarbeit die Zeit im Labor sehr genossen habe. Ich bin sehr froh, dass daraus so schöne Freundschaften entstanden sind. An dieser Stelle möchte ich auch Gregor danken, dafür dass du mir auch nachdem du unser Labor verlassen immer geholfen hast und ein echter Freund warst.

Ein großes Dankeschön geht an alle ehemaligen und derzeitigen Mitglieder der AG Stülke, AG Commichau und AG Rismondo. Ich hatte immer eine tolle Zeit im Labor und dazu hat die schöne Atmosphäre in der Arbeitsgruppe zu einem großen Teil beigetragen.

Mein allergrößter Dank geht aber an meine Eltern Bettina und Volker Krüger, meinen Bruder Marius und an Daniel. Ich danke euch für die Selbstverständlichkeit, mit der ihr mich zu jeder Zeit bedingungslos unterstützt habt. Nur durch euren Rückhalt konnte ich mich immer unbesorgt auf meine Forschung konzentrieren. Dafür bin ich euch unendlich dankbar und deswegen möchte ich Euch diese Arbeit widmen. 


\section{List of publications}

Krüger, L., Herzberg, C., Rath, H., Pedreira, T., Poehlein, A., Gundlach, J., Daniel, R., Mäder, U., Stülke, J. (2020) Essentiality of c-di-AMP in Bacillus subtilis: Bypassing mutations converge in potassium and glutamate homeostasis. bioRxiv.

Krüger, L., Herzberg, C., Wicke, D., Bähre, H., Heidemann, J. L., Dickmanns, A., Schmitt, K., Ficner, R., Stülke, J. (2020) A rendezvous of two second messengers: The c-di-AMP receptor protein DarB controls (p)ppGpp synthesis in Bacillus subtilis. bioRxiv.

Stülke. J. and Krüger, L. (2020) Cyclic di-AMP signaling in bacteria. Annual Review of Microbiology. 74:159-179.

Klewing, A., Koo, B., Krüger, L., Poehlein, A., Reuß, D., Daniel, R., Gross, C., Stülke, J. (2020) Resistance to serine in Bacillus subtilis: Identification of the serine transporter YbeC and of a metabolic network that links serine and threonine metabolism. Environmental Microbiology. 22: 3937-3949.

Krüger, L., Herzberg, C., Warneke, R., Poehlein, A., Stautz, J., Weiß, M., Daniel, R., Hähnelt, I., Stülke, J. (2020) Two ways to convert a low- to a high-affinity potassium channel: Control of Bacillus subtilis KtrCD. Journal of Bacteriology. 202:e00138-20.

Gundlach, J., Krüger, L., Herzberg, C., Turdiev, A., Poehlein, A., Tascón, I., Weiss, M., Hertel, D., Daniel, R., Hänelt, I., Lee, V. T., Stülke, J. (2019) Sustained sensing in potassium homeostasis: Cyclic di-AMP controls potassium uptake by KimA at the levels of expression and activity. Journal of Biological Chemistry. 294:9605-9614. 


\section{Table of content}

Chapter 1 - Abstract/ Zusammenfassung …...................................................... 1

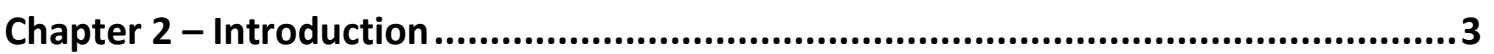

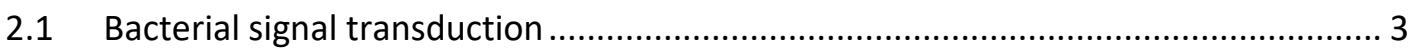

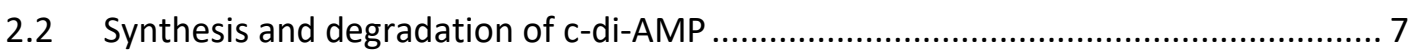

2.3 The importance of understanding the role of c-di-AMP targets............................... 10

2.4 Networking in the second messenger world....................................................... 13

2.5 Potassium and glutamate - the most abundant ions ............................................ 14

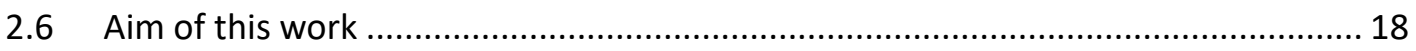

Chapter 3 - Regulation of potassium uptake by KimA........................................19

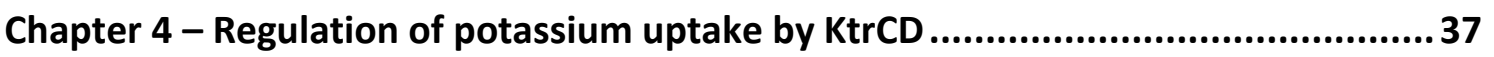

Chapter 5 - Bypassing cyclic di-AMP essentiality ............................................55

Chapter 6 - Nucleotide second messenger crosstalk ........................................ 83

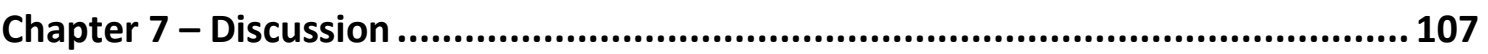

7.1 c-di-AMP and its role in maintaining potassium homeostasis ............................. 107

7.2 The connection between c-di-AMP, potassium and glutamate............................. 111

7.3 New c-di-AMP functions and targets ................................................................ 114

7.4 c-di-AMP and (p)ppGpp crosstalk is mediated by DarB ........................................ 118

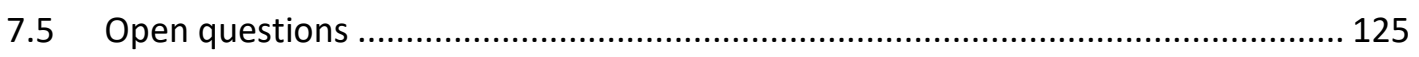

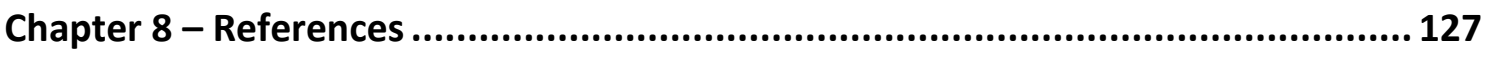

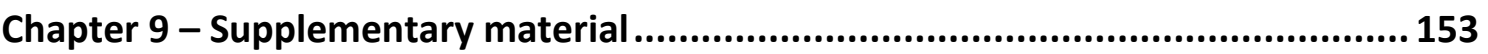

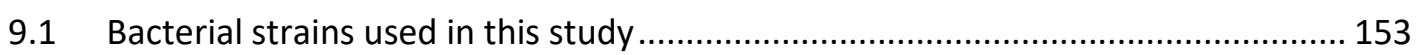

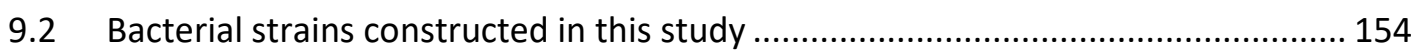

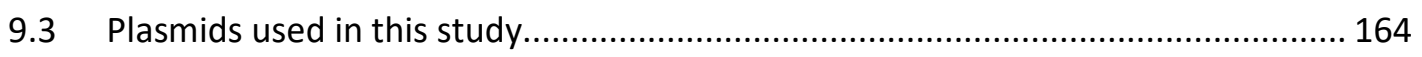

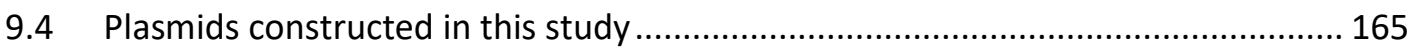




\section{Chapter 1 - Abstract/ Zusammenfassung}

Bacillus subtilis, as a soil inhabitant, has to adapt to rapidly changing osmotic conditions. The response to these osmotic changes represents one of the most critical issues in the lifecyle of bacteria, and the tight regulation of the responsive pathways is of particular importance to guarantee survival. The nucleotide second messenger cyclic di-AMP is involved in the response to changes in the external salinity as it plays a pivotal role in the control of the uptake of potassium ions. To guarantee tight regulation, c-di-AMP controls the two high-affinity potassium importers KtrAB and KimA on both transcriptional and protein level. The low-affinity potassium channel KtrCD, however, is controlled by the nucleotide on protein level only. In this study we revealed a second mode of regulation and showed that KtrCD is converted into a high-affinity potassium channel in the presence of glutamate. On the other hand, the affinity of the glutamate transporter GItT for glutamate depends on the availability of potassium ions. This mutual regulation highlights the importance of maintaining the balance of the two counterions and gives c-di-AMP a special role in monitoring the ionic strength of the cytoplasm. This is supported by our observation that not only potassium, as previously reported, but also glutamate is toxic for a strain lacking the nucleotide second messenger. In this study we isolated suppressor mutants that allow growth of a c-di-AMP-free strain exposed to glutamate. Characterization of these suppressors revealed that they contain pairs of mutations affecting glutamate and potassium homeostasis, phospholipid biosynthesis, motility, and respiration. Several independent mutations affected $y b e C$ and the protein was identified as a novel glutamate transporter and renamed to AimA (amino acid importer $\underline{\mathrm{A}}$ ). The diversity of suppressor mutations suggests that the second messenger likely impacts other cellular processes in addition to the homeostasis of potassium and glutamate. Moreover, we could show that the response to potassium limitation includes the small signal transduction protein DarB (c-di-AMP receptor $\underline{B}$ ). DarB is conserved among Firmicutes but its function has remained elusive until now. Low extracellular potassium levels lead to the presence of the apo-form of DarB. This ligand-free version of the protein binds the dual function (p)ppGpp synthetase/hydrolase Rel and activates ribosome-independent (p)ppGpp synthesis. Accumulation of (p)ppGpp activates the stringent response which induces a global cellular reprogramming, including the downregulation of ribosomal activities. This becomes important under extreme potassium limitation as potassium ions display crucial components of the active ribosome. The regulation of translational processes by a c-di-AMP-receptor through activation of the stringent response displays a novel event of second messenger crosstalk. Together with the suppressor analysis this work demonstrates a central role of c-di-AMP in the control of potassium and glutamate homeostasis, which is linked to the stringent response in $B$. subtilis. 
Bacillus subtilis muss sich als Bodenbewohner an sich rasch verändernde osmotische Bedingungen anpassen. Die Reaktion auf diese osmotischen Veränderungen stellt ein kritisches Problem im Lebenszyklus von Bakterien dar, und die strenge Regulierung der Reaktionswege ist von essentieller Bedeutung. Der Nukleotid-Sekundärbotenstoff zyklisches di-AMP ist an der Reaktion auf Veränderungen des externen Salzgehalts beteiligt, da er eine zentrale Rolle bei der Kontrolle der Kaliumionenaufnahme spielt. Um eine genaue Regulation zu gewährleisten, kontrolliert c-di-AMP die beiden hochaffinen Kaliumimporter KtrAB und KimA sowohl auf Transkriptions-, als auch auf Proteinebene. Der niedrigaffine Kaliumkanal KtrCD wird jedoch nur auf Proteinebene durch das Nukleotid kontrolliert. In dieser Arbeit deckten wir einen zweiten Modus der Regulation auf und zeigten, dass KtrCD in Gegenwart von Glutamat in einen hochaffinen Kaliumkanal umgewandelt wird. Außerdem hängt die Affinität des Glutamat-Transporters GltT für Glutamat von der Verfügbarkeit von Kaliumionen ab. Diese gegenseitige Regulation unterstreicht die Bedeutung der Aufrechterhaltung des Gleichgewichts der beiden Gegenionen und verleiht c-di-AMP eine besondere Rolle bei der Überwachung der lonenstärke des Zytoplasmas. Dies wird durch unsere Beobachtung unterstützt, dass nicht nur Kalium, wie bereits bekannt, sondern auch Glutamat für einen c-di-AMP-freien Stamm toxisch ist. Hier wurden Suppressormutationen charakterisiert, die das Wachstum eines solchen Stammes in Gegenwart von Glutamat ermöglichen. Diese Untersuchung ergab, dass sie Paare von Mutationen enthalten, die die Glutamat- und Kaliumhomöostase, die Phospholipidbiosynthese, die Motilität und die Atmung beeinflussen. Mehrere unabhängige Mutationen beeinflussten ybe $C$ und das Protein wurde als neuer Glutamat-Transporter identifiziert und in AimA ( umbenannt. Die Vielfalt der Suppressormutationen lässt vermuten, dass der Botenstoff neben der Homöostase von Kalium und Glutamat auch andere zelluläre Prozesse beeinflusst. Darüber hinaus konnten wir zeigen, dass die Reaktion auf die Kaliumlimitation das kleine Signaltransduktionsprotein

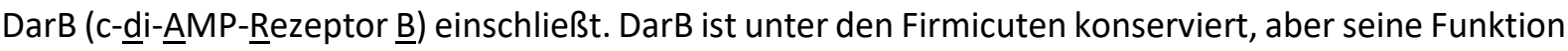
war bisher unbekannt. Niedrige extrazelluläre Kaliumspiegel führen zum Vorhandensein der Apo-Form von DarB. Diese Form des Proteins bindet die (p)ppGpp-Synthetase/Hydrolase Rel und aktiviert die ribosomenunabhängige (p)ppGpp-Synthese. Die Akkumulation von (p)ppGpp aktiviert die stringente Reaktion, die eine globale zelluläre Reprogrammierung einschließlich der Herunterregulierung der ribosomalen Aktivitäten auslöst. Dies wird bei extremer Kaliumlimitation wichtig, da Kaliumionen entscheidende Komponenten des aktiven Ribosoms darstellen. Die Regulation translationaler Prozesse durch c-di-AMP-Rezeptoren durch Aktivierung der stringenten Antwort ist ein neues Beispiel des Second Messenger-Crosstalks. Zusammen mit der Suppressoranalyse zeigt diese Arbeit eine zentrale Rolle von c-di-AMP bei der Kontrolle der Kalium- und Glutamathomöostase auf, die mit der stringenten Reaktion in B. subtilis verbunden ist. 


\section{Chapter 2 - Introduction}

\subsection{Bacterial signal transduction}

Signal transduction processes allow bacteria to sense environmental changes and transduce them into a cellular response. With this powerful ability at hand, they are able to adapt to and resist a broad range of harsh conditions. Responses to different stresses involve distinct signal transduction processes. Some of them are conserved among bacteria, others are more specific for certain prokaryotes. What all signal transduction processes have in common is that they sense an environmental signal and respond to it by altering gene expression or protein activity. Signal transduction can be achieved via sensor proteins, for example by chemoreceptors, alternative sigma factors, or via protein phosphorylation. Two-component systems display a common way to transduce external signals via protein phosphorylation. They consist of two proteins, a histidine kinase and a response regulator. The histidine kinase autophosphorylates in response to a certain signal and transfers the phosphate group to the response regulator, which activates a certain downstream effector (Goudreau and Stock, 1998). Another way to transduce signals is via second messengers. Second messengers are molecules used by the cell to transduce a specific signal from the site of recognition to the target in the cell that responds to the presence of the signaling molecule. This triggers an immediate response which initiates physiological reprogramming to create the most suitable adjustment. This regulatory control can be achieved on every imaginable level, ranging from control of gene expression, to regulation on protein level by direct binding to the effector protein, or via binding to so called signal transduction proteins (see Fig. 2.1). These signal transduction proteins bind the second messenger with a very high affinity and fulfil a specific function in response to the availability of this molecule. They are neither catalytically active on their own, nor does binding of the second messenger to them trigger a direct response. Instead, they simply sense the second messenger and regulate another enzyme or transporter. Nucleotide second messengers are synthesized by nucleotidyl cyclases, like adenylate, diadenylate and diguanylate cyclases in response to an environmental stimulus (Galperin, 2018). Phoshodiesterases degrade the nucleotides and by this allow the regulation of the intracellular nucleotide pool and accordingly the cellular response.

Second messengers that bacteria use for communication are often linear and cyclic nucleotides. Why nucleotides are used for such process and not any other molecule or metabolite, can be explained by the constant availability of nucleotides in the cytosol. From the economical point of view, it appears reasonable to use compounds that can be synthesized from available substrates, and that can be degraded easily by cleavage of the phosphodiester bond (Nelson and Breaker, 2017). Additionally, the degradation of these nucleotide-based second messengers leads to unaltered 
monomers that can be reused directly, also for their original purpose. The preference of ribonucleotides over the 2'-deoxy version of the compound suggests that these signaling molecules likely evolved before DNA emerged (Nelson and Breaker, 2017). The advantage of using cyclic over linear nucleotides is that they are not prone to degradation by exoribonucleases as they are chemically distinctive from RNA degradation products (Nelson and Breaker, 2017).

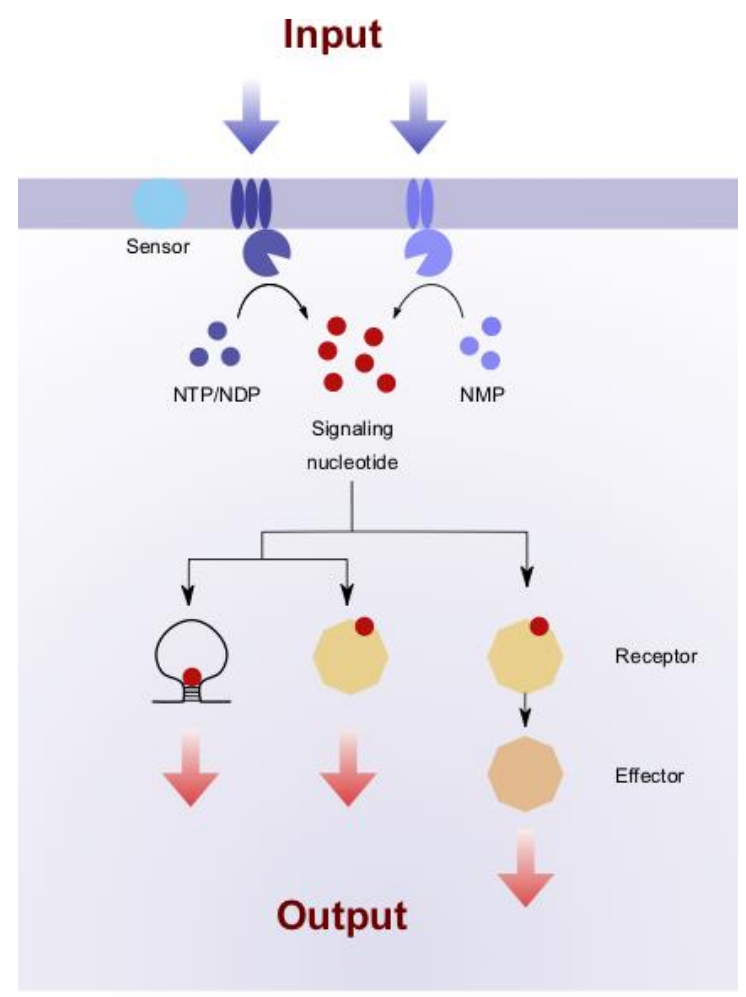

Fig. 2.1 Indirect signal transduction in bacteria. In response to an environmental stimulus, bacteria synthesize or degrade second messenger molecules. This leads to an altered cellular response because the receptors react depending on the availability of the nucleotide. The signal transduction process can happen in a two-step way by direct interaction of the second messenger with a receptor that induces a cellular response. On the other hand, the process can take place via a receptor that on its own is not able to induce a cellular response. This receptor rather interacts with the real effector to transduce the signal and induce the cellular response.

The first cyclic second messenger discovered in bacteria was cyclic AMP (cAMP) in 1965 (Makman and Sutherland, 1965) and its role in carbon catabolite repression is well established (Ullmann and Monod, 1968; Görke and Stülke, 2008). The ability of bacteria to produce cyclic GMP (cGMP) was unclear for some time, however, the production of the second messenger was finally reported in Rhodospirillum centenum and this nucleotide was shown to be involved in developmental processes in this organism (Marden et al., 2011). 


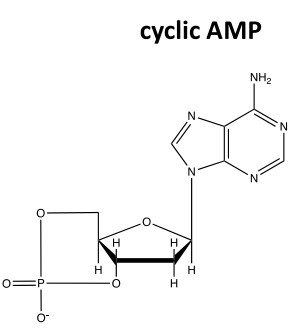

cyclic di-AMP

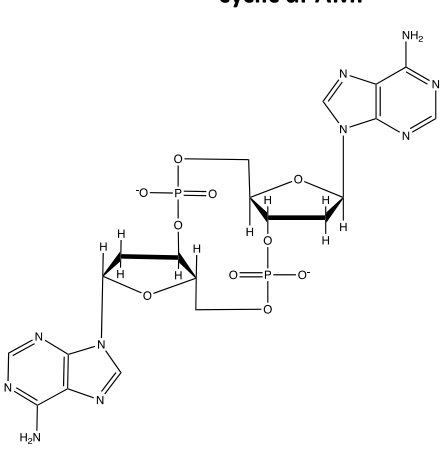

cyclic UMP-AMP

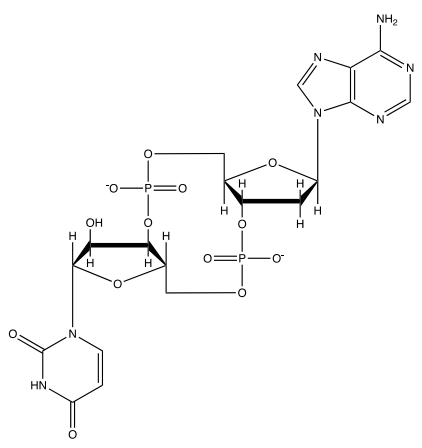

cyclic GMP

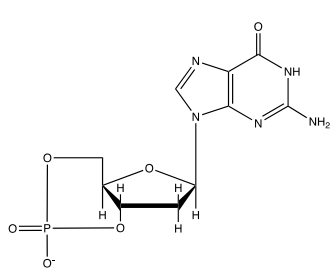

cyclic di-GMP

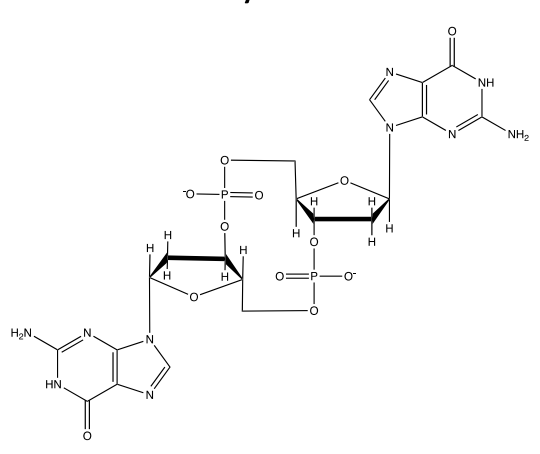

cyclic di-UMP

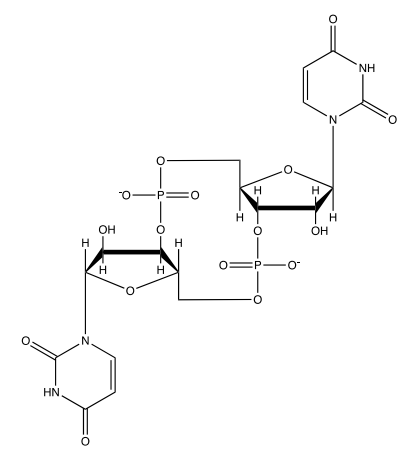

(p)ppGpp

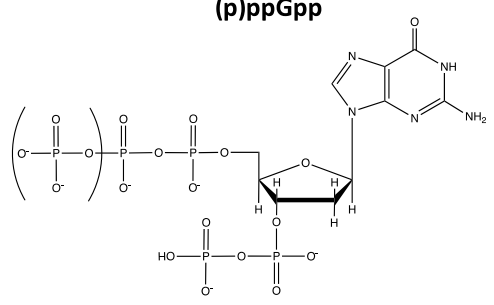

cyclic AMP-GMP

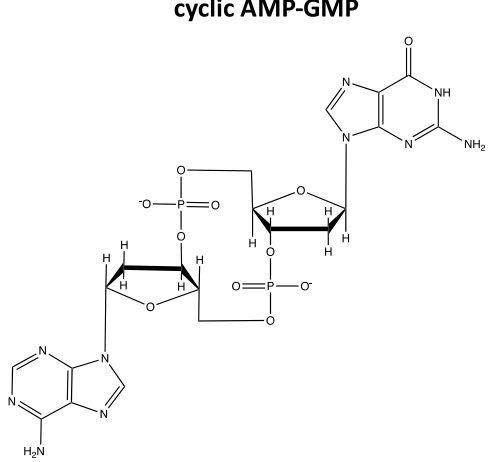

cyclic AMP-AMP-GMP

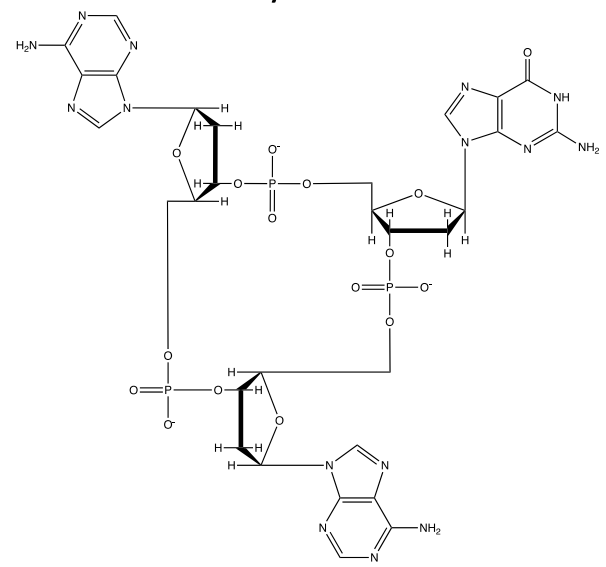

Fig. 2.2. Bacterial nucleotide second messengers. Bacteria use various nucleotide second messengers in distinct signal transduction processes. The first identified nucleotide second messengers are cAMP, cGMP, and the alarmone (p)ppGpp, that are synthesized from a single purine nucleotide. In addition to the purine based cyclic dinucleotides c-di-AMP, c-di-GMP, and c-AMP-GMP, pyrimidine based cyclic nucleotides, as c-UMP-AMP, c-di-UMP were discovered. Additionally, the conjugation state of cyclic nucleotides does not seem to be limited to dinucleotides, as it is shown by the detection of a cyclic trinucleotide molecule, c-AMP-AMP-GMP. In contrast to the classical purine based nucleotides, the physiological function of the novel pyrimidine based molecules and cyclic trinucleotides remains to be elucidated.

One type of second messenger that is used by virtually all bacteria is a hyperphosphorylated nucleoside, guanosine tetra- and penta-phosphate. These linear nucleosides were identified in 1968 and are commonly referred to as (p)ppGpp or alarmone (Cashel and Gallant, 1969). They are worthy to bear the name "alarmone", as the production of this nucleotide in response to starvation induces a 
global metabolic reprogramming in the cell (Cashel and Gallant, 1969; Potrykus and Cashel, 2008; Liu et al., 2015). This includes modulation of growth, transcription, translation and cell cycle (Germain et al., 2013; Mechold et al., 2013; Ross et al., 2013; Hauryliuk et al., 2015; Ronneau and Hallez, 2019). Even though starvation is the major trigger for ( $\mathrm{p}$ )ppGpp production, the nucleoside is also produced in response to a variety of other stresses including variation in the availability of oxygen (Glass et al., 1979), heat stress (Schäfer et al., 2020), and pH downshift (Wells and Gaynor, 2006).

Another class is represented by cyclic dinucleotides, which include the well-studied cyclic diGMP (c-di-GMP) and cyclic di-AMP (c-di-AMP). While c-di-GMP was identified in the late eighties (Ross et al., 1987),c-di-AMP remained hidden until 2008 where it appeared in the crystal structure of the synthesizing enzyme DisA (Witte et al., 2008). By now the role of c-di-GMP is well studied, and the molecule is known to be involved in the lifecycle switch between unicellular motile and multicellular sessile biofilm, thereby playing an important role in the establishment of pathogenicity in several bacteria (Tamayo et al., 2007). In Vibrio cholerae, the second messenger is even directly involved in the expression of virulence genes (Tamayo et al., 2007). Additionally, c-di-GMP plays a crucial role in cell cycle progression in Caulobacter crescentus (Duerig et al., 2009). The roles of c-di-GMP are wideranging and the second messenger itself is widespread in many bacteria. More importantly, the number of enzymes synthesizing and degrading this molecule is huge, and they are found in high numbers, even within the same organism. The roles of c-di-AMP on the other hand are not completely understood and the second messenger seems to be involved in more cellular processes, than initially expected. The number of enzymes synthesizing and degrading it is way lower as compared to c-di-GMP-related enzymes, suggesting that the presence or absence of c-di-AMP needs to be tightly regulated and this will be discussed later. In contrast to second messengers consisting solely of one type of purine moiety, the production of the composite dinucleotide cyclic GMP-AMP ( $3^{\prime}, 3^{\prime}$-cGAMP) was shown to play an important role in the virulence of $V$. cholerae (Dziejman et al., 2002; Davies et al., 2012). The synthesizing enzyme DncV is special in its property to not only produce $3^{\prime}, 3^{\prime}$-cGAMP, but also c-di-GMP and c-di-AMP (Davies et al., 2012). Interestingly, three years ago two independent groups provided evidence, that our knowledge of bacterial second messengers needs to be expanded further, as they revealed the existence of cyclic oligoadenylates (Kazlauskiene et al., 2017; Niewoehner et al., 2017).

It is stunning to note, that all second messengers described above are constructed from purine nucleotides (Fig. 2.2). That our understanding of nucleotide second messengers is still incomplete, was demonstrated by Whiteley et al., who identified a completely novel class of cyclic dinucleotide transferases (Whiteley et al., 2019). The E. coli enzyme CdnE (ㅁGAS/DnncV-like nucleotidyltransferase in E. coli) catalyzes the synthesis of cyclic UMP-AMP (cUMP-AMP), the first hybrid purine-pyrimidine 
cyclic-dinucleotide to be discovered (Whiteley et al., 2019). They expanded the list even further with a homology search and identified 16 new enzymes able to produce various novel cyclic nucleotides, among them c-di-UMP, cCMP-UMP, cUMP-AMP, cUMP-GMP, and even a cyclic tri-nucleotide cyclic AMP-AMP-GMP (CAAG). Even though not every single one of these nucleotides might be formed in vivo, it demonstrates that the bacterial world harbors the ability to offer quite a repertoire of cyclic nucleotides. As these enzymes are encoded on mobile genetic elements and are not part of the core genome, it appeared likely that they might have emerged recently as a result of genomic adaptation (Whiteley et al., 2019).

\subsection{Synthesis and degradation of c-di-AMP}

c-di-AMP is the first second messenger that was shown to be essential in Bacillus subtilis and other Firmicutes (Song et al., 2005; Glass et al., 2006; French et al., 2008; Mehne et al., 2013). However, not only the deletion of the synthesizing enzymes brings in the cell in life-threatening conditions, but also the presence of the degrading enzymes is required to prevent toxic accumulation of the second messenger (Mehne et al., 2013; Ye et al., 2014; Huynh et al., 2015; Gundlach et al., 2015b). This underlines the importance of always sustaining the right amount of c-di-AMP in the cell and explains why c-di-AMP is termed "essential poison" (Gundlach et al., 2015b).

c-di-AMP is synthesized from two molecules of ATP by diadenylate cyclases. These enzymes are characterized by the presence of the DAC (diadenylate çclase) domain (Römling, 2008; Witte et al., 2008). Moreover, they may contain additional domains, that control their enzymatic activity or provide them with secondary abilities, as for instance membrane-anchoring or DNA binding (Römling, 2008; Corrigan and Gründling, 2013; Commichau et al., 2015). Until now, five classes of DACs have been characterized and they are referred to as CdaA, DisA, CdaS, CdaM, and CdaZ (Table 2.1). CdaA and DisA are the most widespread types of diadenylate cyclases in bacteria (Table 2.1; Corrigan and Gründling, 2013). CdaA contains three transmembrane domains, and is regulated by the extracellular oriented protein $\mathrm{CdaR}$, that permanently binds to $\mathrm{CdaA}$ and regulates the activity of the cyclase upon osmotic alterations (Mehne et al., 2013; Commichau et al., 2015; Gibhardt et al., 2020). cdaA and cdaR are encoded in a conserved operon, while in some species they were even found to be translated into one protein. In some species, this operon is expanded by a third gene encoding the phosphoglucosamine mutase GImM (Mehne et al., 2013). GImM was reported to bind to and inhibit the activity of CdaA (Gundlach et al., 2015b; Gibhardt et al., 2020). 
Table 2.1. Distribution of the five classes of diadenylate cyclases. The occurrence of the respective gene in the genome of the species and the experimental verification of the function is indicated by an X. The absence is shown by a dash -

\begin{tabular}{lccccc}
\hline Species (class) & CdaA & DisA & CdaS & CdaM & Cdaz \\
\hline $\begin{array}{l}\text { Bacillus subtilis } \\
\text { (Spore-forming Bacilli) }\end{array}$ & $\mathrm{X}$ & $\mathrm{X}$ & $\mathrm{X}$ & - & - \\
$\begin{array}{l}\text { Listeria monocytogenes } \\
\text { (Non-spore-forming Bacilli) }\end{array}$ & $\mathrm{X}$ & - & - & - & - \\
$\begin{array}{l}\text { Streptococcus agalactiae } \\
\text { (Non-spore-forming Bacilli) }\end{array}$ & $\mathrm{X}$ & - & - & - & - \\
$\begin{array}{l}\text { Streptomyces venezuelae } \\
\text { (Actinobacteria) }\end{array}$ & - & $\mathrm{X}$ & - & - & - \\
$\begin{array}{l}\text { Clostridium difficile } \\
\text { (Clostridia) }\end{array}$ & $\mathrm{X}$ & $\mathrm{X}$ & - & - & - \\
$\begin{array}{l}\text { Mycoplasma pneumoniae } \\
\text { (Mollicutes) }\end{array}$ & - & - & - & $\mathrm{X}$ & - \\
$\begin{array}{l}\text { Synechocystis sp. } \\
\text { (Cyanobacteria) }\end{array}$ & $\mathrm{x}$ & - & - & - & - \\
$\begin{array}{l}\text { Methanocaldococcus jannaschii } \\
\text { (Methanogenic Archaea) }\end{array}$ & - & - & - & - & $\mathrm{X}$ \\
\hline
\end{tabular}

However, the stimulus for this regulation remains elusive. The crystal structure of DisA (ㅁNA integrity scanning protein $\underline{A}$ ) of Thermotoga maritima led initially to the identification of c-di-AMP (Witte et al., 2008). In addition to the DAC domain, DisA contains a DNA-binding helix- hairpin-helix domain that allows the enzyme to scan the DNA for holiday junctions (Witte et al., 2008). Production of the second messenger is triggered by these mismatches that inhibit c-di-AMP synthesis (Witte et al., 2008). In this case, c-di-AMP levels reflect the integrity of the DNA and are a direct checkpoint for entry into sporulation (Bejerano-Sagie et al., 2006; Oppenheimer-Shaanan et al., 2011). The third class of DAC enzymes, CdaS, is limited to sporulating Firmicutes and is only expressed during sporulation (Table 2.1; Rosenberg et al., 2015). The N-terminal coiled-coil domain of CdaS is thought to be involved in oligomerization (Mehne et al., 2014; Zheng et al., 2015), thereby inhibiting the activity of the enzyme. This regulation is, however, not proven for all CdaS enzymes. CdaM is a diadenylate cyclase solely identified in Mycoplasma pneumoniae (Table 2.1; Blötz et al., 2017). This enzyme contains one transmembrane domain that might allow the protein to be anchored to the membrane. The CdaZ enzyme represents the most prominent DAC in Euryarchaeota (Table 2.1). This enzyme is special as it contains a N-terminal pyruvate kinase domain (Römling, 2008). This domain is known to bind ligands like pyruvate and glucose 6-phosphate, but also osmolytes (proline and glycerol) (Fenton et al., 2010; 
Abdelhamid et al., 2019). Apart from the different types of additional functional groups, the complex domain architecture of the different DACs share the ability to be regulated by different factors, ranging from ligand binding to sensing the status of the membrane.

Most bacteria that produce c-di-AMP encode one type of diadenylate cyclases. In B. subtilis, and some other Bacilli, we observe a remarkable phenomenon, the presence of three distinct types of the synthesizing enzymes, CdaA, DisA, and CdaS (Römling, 2008; Mehne et al., 2013). This not only suggests that c-di-AMP signaling plays an indispensable role in these organisms, but also makes B. subtilis a perfect model to study the broad range of physiological effects that c-di-AMP has on cellular physiology.

The nucleotide is not only essential, the accumulation of it can result in growth impairments and, in pathogenic bacteria like Staphylococcus aureus, Listeria monocytogenes, or Streptococcus pneumoniae, virulence defects (Corrigan et al., 2011; Bai et al., 2013; Witte et al., 2013; Huynh et al., 2015). Thus, the degradation of c-di-AMP has to be tightly regulated as well. The nucleotide is degraded by phosphodiesterases. Five classes of PDEs have been described, namely PgpH, GdpP, DhhP/NrnA, $\mathrm{CdnP}$, and AtaC (Table 2.2). Some of them contain only a c-di-AMP-degrading domain, while others have additional domains like transmembrane domains. PgpH-like enzymes degrade c-di-AMP via the HD-type phosphohydrolase domain (Huynh et al., 2015). In contrast, GdpP and DhhP enzymes are characterized by the combination of one DHH and one DHHA1 domain (Rao et al., 2010; Bai et al., 2013; Ye et al., 2014). This already suggests that c-di-AMP metabolism may have evolved autonomously in parallel and that bacteria were able to make use of different types of phosphodiester bond degrading enzymes.

In contrast to the enzymes synthesizing c-di-AMP, the enzymes degrading it, are not necessarily specific for the dinucleotide. At least GdpP was reported to have minor degrading activity against c-di-GMP (Rao et al., 2010). PDEs of the DhhP class have evolved towards having completely different substrates, even though they share the same domain architecture. The minimal organism M. pneumoniae for instance encodes for two DhhP like proteins, but only one of them, PdeM, is able to degrade c-di-AMP (Table 2.2), while the other one, NrnA, has a preference for linear substrates (Blötz et al., 2017). The characterization of CdnP in Streptococcus agalactiae revealed a fourth class of PDE enzymes (Table 2.2; Andrade et al., 2016). CdnP is an extracellular cell wall-anchored phosphodiesterase that, together with NudP degrades c-di-AMP into adenosine (Firon et al., 2014; Andrade et al., 2016). Most Actinobacteria lack classical DHH-DHHA1 domain-containing or HD-type PDEs, and it has remained elusive for quite some time how these bacteria degrade the second messenger. A recent study shed light on this issue and identified the AtaC protein as new type of phosphodiesterases in Streptomyces venezuelae (Table 2.2; Latoscha et al., 2020). While PDEs of the 
PgpH and GdpP type degrade c-di-AMP to 5'-pApA (Rao et al., 2010; Huynh et al., 2015), DhhP, CdnP, and AtaC enzymes degrade the second messenger in a two step reaction to AMP via the linear intermediate 5'pApA (Ye et al., 2014; Manikandan et al., 2014; Latoscha et al., 2020). The fact that the diadenylate cyclases CdaA and CdaM, as well as three out of five classes of the PDEs contain transmembrane domains, suggests that these enzymes might respond to an external signal. This idea is strengthened as the $\mathrm{PgpH}$-type phosphodiesterase contains additional extracellular domains that allow direct contact with the environment (Anantharaman and Aravind, 2003; Huynh et al., 2015).

Table 2.2 Distribution of the five classes of phosphodiesterases. The occurrence of the respective gene in the genome of the species is indicated by an $(X)$, when the function has been experimentally verified by an $X$. The absence is shown by a dash - .

\begin{tabular}{lccccc}
\hline Species (class) & PgpH & GdpP & $\begin{array}{c}\text { DhhP/ } \\
\text { NrnA }\end{array}$ & CdnP & AtaC \\
\hline $\begin{array}{l}\text { Bacillus subtilis } \\
\text { (Spore-forming Bacilli) }\end{array}$ & $\mathrm{X}$ & $\mathrm{X}$ & $\mathrm{X}$ & (X) & - \\
$\begin{array}{l}\text { Listeria monocytogenes } \\
\text { (Non-spore-forming Bacilli) }\end{array}$ & $\mathrm{X}$ & $\mathrm{X}$ & $\mathrm{X}$ & (X) & - \\
$\begin{array}{l}\text { Streptococcus agalactiae } \\
\text { (Non-spore-forming Bacilli) }\end{array}$ & - & $\mathrm{X}$ & - & $\mathrm{X}$ & - \\
$\begin{array}{l}\text { Streptomyces venezuelae } \\
\text { (Actinobacteria) }\end{array}$ & - & - & - & $(\mathrm{X})$ & $\mathrm{X}$ \\
$\begin{array}{l}\text { Clostridium difficile } \\
\text { (Clostridia) }\end{array}$ & - & $\mathrm{X}$ & - & $(\mathrm{X})$ & - \\
$\begin{array}{l}\text { Mycoplasma pneumoniae } \\
\text { (Mollicutes) }\end{array}$ & - & - & $\mathrm{X}$ & - & - \\
$\begin{array}{l}\text { Synechocystis sp. } \\
\text { (Cyanobacteria) }\end{array}$ & $\mathrm{X}$ & - & $\mathrm{X}$ & $(\mathrm{X})$ & - \\
$\begin{array}{l}\text { Methanocaldococcus jannaschii } \\
\text { (Methanogenic archaea) }\end{array}$ & - & - & $\mathrm{X}$ & - & \\
\hline
\end{tabular}

\subsection{The importance of understanding the role of c-di-AMP targets}

The presence or absence of c-di-AMP results in cellular responses that need to be tightly regulated. For this reason, dysregulation of the intracellular c-di-AMP concentration leads to deregulated effector responses that might be toxic to the cell. In order to understand the physiological role of c-di-AMP signaling, it is of fundamental importance to know every target within the cell. Targets can be divided into protein and RNA targets (Stülke and Krüger, 2020). Protein targets can induce the cellular response directly or they can function as signal transduction proteins that transduce the signal to the effector 
(Fig. 2.1). RNA targets, so called riboswitches, regulate the expression of a certain gene in response to ligand binding (Breaker, 2012).

RCK_C (regulator of conductance of $\underline{K}^{+} \underline{C}$-terminal) domains are conserved domains that regulate the gating of ions through ion channels (Jiang et al., 2002; Schrecker et al., 2019). RCK_C domains bind ions like $\mathrm{Na}^{+}, \mathrm{Ca}^{2+}$, or $\mathrm{Mg}^{2+}$, but also nucleotides like AMP, ADP, ATP, NADH, and c-diAMP (Zhang et al., 2001; Jiang et al., 2002; Roosild et al., 2002; Yuan et al., 2003; Kröning et al., 2007; Kong et al., 2012; Corrigan et al., 2013; Pliotas et al., 2017). Binding of c-di-AMP to these domains seems to be conserved among Gram-positive bacteria (Corrigan et al., 2013; Kim et al., 2015; Chin et al., 2015). KtrA and KtrC, the cytosolic partners of the transmembrane proteins KtrB and KtrD, respectively, contain a RCK_C domain and the import of potassium ions is regulated by binding of c-di-AMP to this domain (Kim et al., 2015; Rocha et al., 2019). RCK_C domains are also part of the CabP (TrkH) protein of S. agalactiae and potassium import is inhibited by c-di-AMP binding to this domain (Bai et al., 2014). Additionally, the potassium exporter CpaA binds the nucleotide via the RCK_C domain, potentially increasing potassium efflux (Corrigan et al., 2013; Chin et al., 2015). In addition to proteins involved in potassium transport, the RCK_C domain can also be found in the transcriptional regulator BusR. BusR negatively regulates the expression of the busAB operon, which is needed for the uptake of compatible solutes in S. agalactiae (Devaux et al., 2018b). BusR is inhibited by c-di-AMP which results in uncontrolled busAB expression and toxic accumulation of compatible solutes (Devaux et al., 2018b). RCK_C domains bind c-di-AMP very specifically, as the nucleotide binds to four out of five RCK_C domain containing proteins in B. subtilis and this is also true for other organisms that produce c-di-AMP like S. aureus (Corrigan et al., 2013; Gundlach et al., 2019).

Another conserved c-di-AMP target is the sensor histidine kinase KdpD, which is part of the $\mathrm{KdpD} / \mathrm{KdpE}$ two component system (Corrigan et al., 2013; Moscoso et al., 2016). When potassium levels are low, KdpDE activates expression of the $k d p F A B C$ operon that codes for the high affinity potassium transporter Kdp. C-di-AMP binding to KdpD inhibits the protein and, thus, prevents expression of KdpFABC. The c-di-AMP binding site has been localized to the USP (Unniversal Stress Protein) like domain at the N-terminal part of the protein and c-di-AMP was shown to be a negative regulator of the Kdp system (Corrigan et al., 2013; Moscoso et al., 2016).

In addition to the Ktr/HKT/Kdp family (Durell and Guy, 1999), a second class of bacterial potassium transporters is represented by the Kup/HAK/KT (Kup: $\underline{K}^{+} \underline{u p t a k e) ~ f a m i l y ~(B o s s e m e y e r ~ e t ~ a l ., ~}$ 1989; Quintero and Blatt, 1997; Santa-Maria et al., 1997). The first c-di-AMP target in this second class was identified in Lactococcus lactis, where the potassium transporters KupA and KupB were shown to be regulated by the second messenger (Quintana et al., 2019). A second prominent member of the 
Kup family is KimA ( $\underline{K}^{+} \underline{i m p o r t e r} \underline{\text { A) }}$ (Tascón et al., 2020), and the inhibitory effect of c-di-AMP on KimA has already been demonstrated (Gundlach et al., 2017b; Gundlach et al., 2019).

Moreover, potassium transport is regulated on the level of gene expression via riboswitches. Riboswitches are regulatory parts of an mRNA molecule, that regulate the expression of the genes downstream of the riboswitch depending on the availability of the effector molecule (Mandal and Breaker, 2004; Breaker, 2012). Effector molecules can be vitamins, metabolites, metal ions, as well as nucleotides like second messengers (Breaker, 2012). In addition to well-studied c-di-GMP riboswitches, a recent study identified the $y d a O$ riboswitch as a conserved c-di-AMP target in bacteria (Sudarsan et al., 2008; Nelson et al., 2013). The ydaO riboswitch is conserved in bacteria that encode diadenylate cyclases, indicating a functional relationship (Nelson et al., 2013). This riboswitch is associated with genes involved in cell wall metabolism, osmotic responses, and sporulation (Barrick et al., 2004; Block et al., 2010; Nelson et al., 2013). In B. subtilis, the ydaO riboswitch is localized in the $5^{\prime}$ untranslated regions (UTR) of the ktrAB operon and the kimA (previously ydaO) gene. In other Bacilli like Bacillus cereus or Bacillus pseudomycoides, the riboswitch is additionally localized in the $5^{\prime}$-UTR of the $k d p A B C$ operon (Block et al., 2010). Since this regulation by c-di-AMP on expression level is conserved among different organisms and among the different potassium uptake systems, the contribution of riboswitches to the regulation of potassium uptake systems appears to be a conserved feature. The concept of sustained sensing, the phenomenon of a bacterial nucleotide second messenger to control a biological process at different levels (Orr et al., 2016), can be fully applied to the regulation of the potassium transporters as the regulation happens on both, transcriptional and posttranslational level.

In addition to the conserved RCK_C domain, c-di-AMP binds a second conserved domain, the CBS (ㄷystathionine-beta-synthase) domain. CBS domains are structurally conserved domains that can be found in all kingdoms of life (Ereño-Orbea et al., 2013). Even though they are very similar in their structural arrangement, the sequence conservation within the protein family is low (Bateman, 1997; Baykov et al., 2011; Ereño-Orbea et al., 2013). In most cases they appear in pairs or quads and two CBS domains form a so-called Bateman module (Baykov et al., 2011; Ereño-Orbea et al., 2013). CBS domains are part of transporters, enzymes or transcription factors, and they are known to regulate the associated domains via binding of adenosyl ligands (Baykov et al., 2011). Stand-alone CBS domain proteins are an exception, and the move of action upon ligand binding is not so obvious at the first glimpse. CBS-domains bind various adenosyl-ligands like AMP, ADP, ATP, NADH, AdoMet (S-adenosyl methionine), but also nucleic acids and metal ions (Scott et al., 2004; Jin et al., 2007; Xiao et al., 2007; Aguado-Llera et al., 2010; Lucas et al., 2010; Baykov et al., 2011; Jeong et al., 2013; Ereño-Orbea et al., 2013) and only a small portion binds c-di-AMP. For this reason, it appears more likely, that ancient CBS domains provided a basis to bind adenosyl ligands of any kind, and they evolved towards gaining 
specificity for one ligand. It therefore seems quite insecure to make any predictions about new c-di-AMP targets in the CBS family.

Another c-di-AMP target of yet unknown function is the conserved PII-like Protein DarA/PstA (c- di-AMP receptor $\underline{A}$ ). Because DarA lacks additional functional domains, it can be regarded as a classical signal transduction protein. Even though the crystal structure was solved (Choi et al., 2015; Gundlach et al., 2015a) and the protein was studied in several organisms (Corrigan et al., 2013; Sureka et al., 2014; Choi et al., 2015), the signal transduced by DarA, as well as the effectors regulated by it, remain elusive.

\subsection{Networking in the second messenger world}

Each signal transduction process on its own holds the capacity to regulate various cellular processes. The complexity of these signal transduction networks is reflected by the increasing number of target proteins that are identified, as well as by the huge number of newly emerging second messengers. These, partially highly regulated, signal transduction processes create the basis to allow bacteria to become these extremely adaptable organisms that they are. Having these regulatory processes at hand, it appears very likely that they do not function completely independently, but rather make use of each other.

One possibility is that more than one second messenger is involved in the regulation of one biological process. An alternative strategy would be the direct linking of one signal transduction process to another by modulation of the pool of the respective other nucleotide.

Since (p)ppGpp affects the activity of the RNA polymerase in many organisms, it might not be surprising that the alarmone also indirectly controls cellular processes that are regulated by other second messengers. An example would be the regulation of the glp operon in Borrelia burgdorferi, where c-di-GMP-bound PIzA leads to upregulation of the operon (Zhang et al., 2018), while (p)ppGpp, affecting the action of the RNA polymerase, exerts a negative regulation of $g / p$ expression. This opposing regulation has a considerable impact on cellular metabolism, as these two second messenger decide the fate of glycerol-3-phosphate. Another interesting example occurs in V. cholerae, where both (p)ppGpp and c-di-GMP have an activating effect on the expression of $v p s R$ and $v p s T$, the activators of biofilm formation in this organism (He et al., 2012). Additionally, cAMP activates vpsR, but inhibits vpsT expression. (p)ppGpp affects expression of the two genes via interaction with the stationary-phase sigma factor RpoS, CAMP through interaction with the cAMP receptor protein, and c-di-GMP probably via an unknown mechanism via HapR (Waters et al., 2008). Another link between cAMP and c-di-GMP signaling is shown in Pseudomonas aeruginosa where the crosstalk between these two nucleotides results in a significant downregulation of virulence genes during biofilm infections (Almblad et al., 
2015). These examples highlight to which extent the regulation of one biological process is impacted by more than one second messengers.

What certainly occurs to a lesser extent, is the direct regulation of one signal transduction process by another. Nevertheless, the close interconnection between cAMP and c-di-GMP becomes more and more obvious as it was observed in the predatory bacterium Bdellovibrio bacteriovorus that cAMP controls the activity of a c-di-GMP-degrading phosphodiesterase via binding to a N-terminal cAMP-binding sensory domain (Cadby et al., 2019). In a similar fashion it has been observed that the alarmone (p)ppGpp has an inhibiting effect on the phosphodiesterases PgpH and GdpP that degrade the second messenger c-di-AMP in several Gram-positive bacteria (Rao et al., 2010; Huynh et al., 2015; Corrigan et al., 2015). Moreover, the presence of c-di-AMP-responsive riboswitches in front of genes encoding a GGDEF-domain containing enzyme in several organisms, allows the speculation that c-di-AMP has a regulatory function in the expression of c-di-GMP synthesizing enzymes (Nelson et al., 2013). The impact of cAMP on c-di-GMP synthesizing and degrading enzymes as well as the regulation of c-di-AMP degrading enzymes by (p)ppGpp is observed in several bacteria, and it shows how complex and likewise common the cross-talk between different signal transduction processes appears to be. While c-di-GMP acts as the decision-maker between motility and sessility and by this determines the lifestyle of a bacterial cell, the role of c-di-AMP in serious issues such as osmotic homeostasis, however, is essential for life. For this reason, expanding our knowledge on which impact the essential molecule c-di-AMP has on other signal transduction processes is of particular importance. This will allow us in the future to learn more about essential cellular processes and their regulation by second messengers.

\subsection{Potassium and glutamate - the most abundant ions}

The influence of c-di-AMP on the transport of the most abundant cation has been reported for many organisms (Corrigan et al., 2013; Kim et al., 2015; Chin et al., 2015; Moscoso et al., 2016; Gundlach et al., 2017b; Rocha et al., 2019; Quintana et al., 2019). This process of sustained sensing implies the tight regulation of the importers on transcriptional, as well as on protein level, and of the exporters on protein level. This allows the cell to strictly control potassium homeostasis on any level. Potassium ions are essential for life and cells of any kind enrich this alkali metal. The potassium ion maintains the electrochemical potential, osmotic homeostasis, and it buffers the negative charge of the DNA backbone (Epstein, 2003). The reason why all life on earth seems to be based on potassium, remains a mystery, as one would guess sodium ions were abundant in the same manner. Thinking about the origin of life, it seems like an established fact that evolution started in the deep sea before the first creatures colonized the land. The main cation in sea water is certainly sodium, so the reasonable question arises, why nature, under these conditions, would choose potassium over sodium (Danchin 
and Nikel, 2019). There are at least two ways out of this mysterious situation. First, life could have evolved elsewhere, at some place where potassium is the most prominent cation. Secondly, potassium ions were actively chosen over sodium ions, because potassium ions provide some qualities that can exclusively be fulfilled by them (Danchin and Nikel, 2019). When comparing the physico-chemical properties of the two ions, it becomes obvious that they share some properties, but they also differ in some, and those might be the crucial points why potassium became the major intracellular cation. While sodium and potassium ions share the positive charge, they differ in size. Sodium ions have a smaller ionic radius but a larger hydrated radius (Nightingale, 1959). In 1888, Hofmeister claimed for the first time that ions have different effects on protein stability. He observed that sodium ions destabilize ("salt out") egg white proteins more effectively than potassium ions and developed the "Hofmeister series" where he classified ions according to their ability to salt out proteins (Hofmeister, 1888; Xie and Gao, 2013). In agreement with this, sodium ions bind the carboxyl-groups of the protein surface at least twice as strong as potassium ions (Vrbka et al., 2006). This can impair protein function and might be the reason why the major intracellular cation is potassium and not sodium (Vrbka et al., 2006). In addition, potassium ions are more effective in peptide bond formation than sodium ions (Dubina et al., 2013). This is further supported by the fact that cells have special sodium export systems to pump the ion out of the cell. Moreover, potassium's ability to maintain the solubility of cellular components together with the major intracellular anions carboxylate and phosphate is greater than that of sodium (Collins, 1997; Danchin and Nikel, 2019). These reasons argue in favor of a selection of potassium over sodium ions. However, the question how this is compatible with the cradle of life, remains to be answered in the future.

Many protein complexes, and most importantly the heart of a cell, the translation machinery, need potassium ions for functionality. Potassium ions are arranged all over the ribosome and they were reported to play a role in translation elongation. In agreement with this, the number of potassium ions in the ribosome changes at different functional stages. While the initiation complex harbors 127 potassium ions, in the elongation complex, the number increases to 211 potassium ions (Rozov et al., 2019). This suggests that potassium ions are required for translation elongation and are therefore crucial for protein synthesis. Indeed, potassium ions are needed for stabilization of the functional centers of the ribosome, by stabilizing the mRNA, tRNA, as well as for stabilizing all three ribosomal RNAs (Rozov et al., 2019). Contrary to the activating effect of potassium ions, sodium ions exhibit an inhibitory effect on ribosomal activities (Lubin and Ennis, 1964; Miskin et al., 1970). In the early sixties it has already been shown that in potassium depleted cells, the potassium ions are replaced by sodium ions which immediately blocked protein synthesis (Lubin and Ennis, 1964; Ennis and Artman, 1972). Moreover, potassium ions are indispensable for counterbalancing the negative charge of the largest 
nucleic acid molecule in the cell, the chromosome. Unlike previously thought, this process was finally shown to be highly specific and potassium ions, as well as sodium ions, were shown to bind to sequence-specific sites in the major and minor groove of the DNA and these monovalent binding sites on the DNA were described as flexible ionophores (Hud and Polak, 2001; Cheng et al., 2006; Auffinger et al., 2016). The fact that potassium ions are associated with nucleic acid processes, like ribosome formation, as well as the integrity of the chromosome, underlines the crucial and well-established role of this cation for essential cellular processes and osmotic homeostasis. It is interesting to note, that while potassium appears to be the most abundant intracellular cation, the extracellular environment is dominated by sodium. In agreement with this, extracellular thrombin relies on sodium ions, while a range of intracellular enzymes are known to be potassium dependent, among others the pyruvate kinase (Nowak and Suelter, 1981), the aldehyde dehydrogenase (Garza-Ramos et al., 2013), and the pyridoxal kinase (Gohara and Di Cera, 2016).

It is well established that the counterion of potassium is glutamate (McLaggan et al., 1990; McLaggan et al., 1994). The requirement for enriching glutamate can be explained by the nature of glutamate to serve as the amino group donor for every single amino acid. The synthesis of each amino acid requires at least one specific aminoacyltransferase and these enzymes have a rather low affinity for glutamate as their substrate. Hence, maintaining high amounts of glutamate in the cell is crucial for unimpeded protein synthesis. For this reason, cells might have evolved towards combining this already high glutamate level to maintenance of ion homeostasis in the cell. From the total metabolite pool, around $40 \%$ can be attributed to glutamate leading to a total glutamate concentration of around 100-120 mM (Bennett et al., 2009) under normal conditions, but concentrations of up to $160 \mathrm{mM}$ are reported for cells encountering osmotic upshock (Whatmore et al., 1990). This is accompanied by potassium concentrations between 200 and $400 \mathrm{mM}$ (Epstein, 2003) depending on the availability in the environment. While glutamate is available in the cytoplasm, potassium ions are present as free and bound ions. Bound potassium ions can be regarded as bound to macromolecular anions and free potassium ions counterbalance the charge of small anions (McLaggan et al., 1994). This explains why the intracellular concentrations of the two counterions potassium and glutamate are not the same.

In contrast to potassium that needs to be enriched from the environment under any given condition, glutamate can be both, taken up and synthesized within the cell. Two distinct glutamate synthesis pathways are described, that are present in the model organisms Escherichia coli and B. subtilis. E. coli uses the single enzyme glutamate dehydrogenase (GDH) to synthesize glutamate from one molecule of 2-oxoglutarate and one molecule ammonium in a reversible reaction. B. subtilis harbors a two-step pathway consisting of the two enzymes glutamate synthase (GOGAT) and glutamine synthetase (GS). Two molecules of glutamate are formed from one molecule of 
2-oxoglutarate and one molecule of glutamine by the GOGAT. Glutamine is produced by the GS from the fixation reaction of one molecule of ammonium to one molecule of glutamate. With this, $B$. subtilis evolved a very efficient way of glutamate production that guarantees the synthesis also at low ammonium concentrations as the GS, in contrast to the GDH, has a high affinity for its substrate (Reitzer, 2003). However, even if the pathways might look like they would fulfil the same need, several organisms like E. coli, Corynebacterium glutamicum, Salmonella typhimurium, and Synechocystis sp. (Ikeda et al., 1996; Chávez et al., 1999; Tesch et al., 1999; Reitzer, 2003; Rehm and Burkovski, 2011) encode for both of these pathways. The option of having two possibilities to synthesize glutamate underlines the undisputed importance of glutamate synthesis for the cell, and gives credit to a selective advantage of having two opportunities instead of one (Chávez et al., 1999). When the precursors for glutamate synthesis become limiting, the cells are also able to accumulate the metabolite from their environment. In order to achieve this, cells are equipped with glutamate uptake systems with different properties, regarding their activity, affinity for the substrate, as well as the nature of the cotransported ion. Moreover, glutamate plays an important role in the osmotic stress response. Glutamate serves as a precursor for the biosynthesis of proline, a compatible solute that accumulates under hyperosmotic conditions and prevents the cell from bursting. Among other osmolytes, $B$. subtilis produces glycine betaine and proline de novo (Whatmore et al., 1990). It is reported from many organisms, that the bacterial response to hyperosmotic conditions is initiated by a rapid uptake of potassium ions (Whatmore et al., 1990; McLaggan et al., 1994; Holtmann et al., 2003). Because these extremely high potassium concentrations are harmful for the cell in the long term, the cells start to replace potassium ions with compatible solutes to decrease the ionic strength of the cytoplasm (Whatmore et al., 1990). Synthesis of glutamate allows for scaled-up production of proline that accumulates to up to $700 \mathrm{mM}$ inside the cell (Whatmore et al., 1990). This describes a conserved mechanism for the adaptation to changing osmotic conditions in the environment. The interconnection between potassium and glutamate through all domains of life is well established, the exact mechanisms achieving the balance between these most abundant ions, however, remain partially unexplored. 


\subsection{Aim of this work}

After the nucleotide second messenger c-di-AMP has been discovered in 2008 (Witte et al., 2008), the signaling pathway was studied in several bacterial and archaeal species. While our knowledge about this pathway constantly increases, there are still essential open questions. Those include the control of the intracellular nucleotide level, the signals this pathway responds to, and the function of known and unknown receptor proteins.

The control of potassium homeostasis has previously been observed to be one of the essential functions of the nucleotide (Gundlach et al., 2017b). The aim of this thesis is to gain deeper knowledge about this process and to find out which role glutamate, the counterion of potassium, plays in this scenario. B. subtilis encodes two known c-di-AMP-binding signal transduction proteins, DarA and DarB. The homologs of DarA and DarB in L. monocytogenes are targets for mutations when a $\Delta$ dac strain is cultivated on complex medium (Whiteley et al., 2015). This indicates a toxic function of the apoproteins and raises the question which essential processes might be regulated by these small signal transduction proteins. As these mutations were always accompanied by mutations dealing with the increased osmotic pressure of a $\Delta d a c$ strain (Whiteley et al., 2015), it appears likely that these two proteins are involved in other yet unidentified pathways. In order to shed light on another aspect of this signaling pathway, next to its role in the regulation of osmotic processes, the present work addresses the c-di-AMP receptor DarB. 


\section{Chapter 3 - Regulation of potassium uptake by KimA}

The results described in this chapter were originally published in Journal of Biological Chemistry (doi: 10.1074/jbc.RA119.008774):

\section{Sustained sensing in potassium homeostasis: Cyclic di-AMP controls potassium uptake by KimA at the levels of expression and activity}

Jan Gundlach ${ }^{1}$, Larissa Krüger ${ }^{1}$, Christina Herzberg ${ }^{1}$, Asan Turdiev², Anja Poehlein ${ }^{3}$, Igor Tascón ${ }^{4}$, Martin Weiß ${ }^{1}$, Dietrich Hertel ${ }^{5}$, Rolf Daniel ${ }^{3}$, Inga Hänelt ${ }^{4}$, Vincent T. Lee ${ }^{2}$, and Jörg Stülke ${ }^{1}$

\footnotetext{
${ }^{1}$ Department of General Microbiology, GZMB, Georg-August-University Göttingen, Germany

${ }^{2}$ Department of Cell Biology and Molecular Genetics, University of Maryland, College Park, MD, USA

${ }^{3}$ Department of Genomic and Applied Microbiology, GZMB, Georg-August-University Göttingen, Germany

${ }^{4}$ Institute of Biochemistry, Goethe University Frankfurt, Germany

${ }^{5}$ Department of Plant Ecology and Ecosystems Research, Georg-August-University Göttingen, Germany
}

\section{AUTHOR CONTRIBUTIONS}

JS conceived the study. IH, VTL, and JS designed the experiments. JG, LK, CH, AT, AP, IT, MW, DH, and $\mathrm{RD}$ carried out the experiments. JG and $\mathrm{CH}$ prepared the plasmids for the DRaCALA experiments and performed the suppressor isolation of the c-di-AMP-free strain. LK cloned the KimA mutant protein and conducted the kinetic experiments with wild type KimA and the KimA mutant protein. AT and VTL conducted and analyzed the DRaCALA experiments. IT studied the direct effect of c-di-AMP on KimA by growth assays. AP and RD did the whole genome sequencing. MW performed the experiments on the potential interaction of DarA and KimA. IH and JS wrote the manuscript. All authors approved the manuscript. IH and JS acquired funding. IH and JS provided supervision. 


\section{ABSTRACT}

The signaling nucleotide cyclic di-AMP (c-di-AMP) is the only known essential second messenger in bacteria. Recently, c-di-AMP has been identified as being essential for controlling potassium uptake in the model organism Bacillus subtilis and several other bacteria. A B. subtilis strain lacking c-di-AMP is not viable at high potassium concentrations, unless the bacteria acquire suppressor mutations. In this study, we isolated such suppressor mutants and found mutations that reduced the activities of the potassium transporters KtrCD and KimA. Although c-di-AMP-mediated control of KtrCD has previously been demonstrated, it is unknown how c-di-AMP affects KimA activity. Using the DRaCALA screening assay, we tested for any interactions of KimA and other potential target proteins in B. subtilis with c-di-AMP. This assay identified $\mathrm{KimA}$ as well as the $\mathrm{K}^{+} / \mathrm{H}^{+}$antiporter $\mathrm{KhtT}$; the potassium exporter CpaA (YjbQ); the osmoprotectant transporter subunit OpuCA; the primary $\mathbf{M g}^{2+}$ exporter MgtE; and DarB (YkuL), a protein of unknown function, as bona fide c-di-AMP-binding proteins. Further, binding of c-di-AMP to KimA inhibited potassium uptake. Our results indicate that c-di-AMP controls KimA-mediated potassium transport at both kimA gene expression and KimA activity levels. Moreover, the discovery that potassium exporters are c-di-AMP targets indicates that this second messenger controls potassium homeostasis in B. subtilis at a global level by binding to riboswitches and to different classes of transport proteins involved in potassium uptake and export.

\section{INTRODUCTION}

The essential signaling nucleotide c-di-AMP is a recently discovered second messenger that is produced by many bacteria and some archaea (Witte et al., 2008; Commichau et al., 2018b). The reasons for the essentiality of this dinucleotide have long remained elusive. Recent studies with the Gram-positive bacteria Listeria monocytogenes, Bacillus subtilis, Staphylococcus aureus, and Streptococcus agalactiae revealed that c-di-AMP becomes dispensable, if the bacteria are cultivated on strictly controlled minimal media (Whiteley et al., 2015; Gundlach et al., 2017b; Zeden et al., 2018; Devaux et al., 2018b). In the Gram-positive model organism B. subtilis, c-di-AMP is dispensable only at low potassium concentrations in minimal medium (Gundlach et al., 2017b).

Binding assays to search for target proteins of the molecule revealed that c-di-AMP binds several different proteins, with the majority being involved in potassium and compatible solute uptake (see Commichau et al. 2018 for review). c-di-AMP binds to the conserved RCK_C (regulator of conductance of $\mathrm{K}^{+}$) domains in the gating components of potassium channels (Corrigan et al., 2013; Bai et al., 2014; Blötz et al., 2017; Devaux et al., 2018b; Quintana et al., 2019). Moreover, c-di-AMP binds and inhibits the unrelated Kup potassium transporters in L. lactis (Quintana et al., 2019). Additionally, c-di-AMP controls the expression of different potassium uptake systems. It binds to the 
KdpD sensor kinase that controls the expression of the S. aureus and L. monocytogenes Kdp potassium transport systems and to the two copies of the c-di-AMP (formerly ydaO) riboswitch that controls the expression of the high affinity potassium uptake systems KtrAB and KimA in B. subtilis and other bacteria (Nelson et al., 2013; Moscoso et al., 2016; Gundlach et al., 2017b). This makes c-di-AMP the only known second messenger that controls a single biological process by binding both to a protein and to the corresponding mRNA. Similarly, the uptake of osmoprotectants is regulated at levels both of gene expression and protein activity. c-di-AMP binds to the RCK_C domain of the transcription repressor BusR, which controls the expression of the busAB operon for the transport of compatible solutes in lactic acid bacteria (Devaux et al., 2018b; Pham et al., 2018). Moreover, c-di-AMP binding to the regulatory CBS domain of the ATP-binding subunit directly inhibits osmoprotectant uptake systems in S. aureus and L. monocytogenes (Schuster et al., 2016; Huynh et al., 2016).

Recently, the concept of sustained sensing has been proposed for second messengers that control a biological process by binding multiple targets (Orr et al., 2016). The control of potassium and compatible solute transport nicely fits this concept. In addition to these processes, c-di-AMP controls the entry to the citric acid cycle by binding to the pyruvate carboxylase of L. monocytogenes and L. lactis (Sureka et al., 2014; Choi et al., 2017). Finally, DarA, a Pll-like protein of unknown function binds c-di-AMP in B. subtilis, S. aureus, and L. monocytogenes (Corrigan et al., 2013; Sureka et al., 2014; Gundlach et al., 2015a), and CbpB, an unknown protein consisting of two CBS domains were identified as c-di-AMP-binding protein in L. monocytogenes (Sureka et al., 2014).

In B. subtilis, c-di-AMP is essential for potassium homeostasis (Gundlach et al., 2017b). However, only very few target molecules of the dinucleotide are known for this model organism. In a first screen for c-di-AMP targets in B. subtilis only one protein could be identified: the PII-like signal transduction protein DarA showed high and specific affinity for c-di-AMP, although its function has remained enigmatic (Gundlach et al., 2015a). The other known c-di-AMP target proteins of B. subtilis are the RCK_C domains of the peripheral membrane proteins $\mathrm{KtrA}$ and $\mathrm{KtrC}$, which are the regulatory subunits of the KtrAB and KtrCD complexes, respectively (Albright et al., 2006; Corrigan et al., 2013; Rocha et al., 2019). For KtrA and KtrC from different organisms, it has been shown that upon binding of c-di-AMP the complexes are inhibited, most likely due to conformational changes within the regulatory subunits (Vieira-Pires et al., 2013; Bai et al., 2014; Rocha et al., 2019).

As mentioned above, the control of potassium homeostasis is a key function of c-di-AMP and this function is crucial for the essentiality of the second messenger. Thus, it is tempting to speculate that c-di-AMP might regulate further potassium transport systems. Besides the high- and low-affinity potassium uptake systems KtrAB and KtrCD (Holtmann et al., 2003), respectively, recently the highaffinity potassium importer KimA was discovered (Gundlach et al., 2017b; Gundlach et al., 2017a). The 
expression of both high-affinity uptake systems is controlled by a c-di-AMP responsive riboswitch (Nelson et al., 2013; Gundlach et al., 2017b), but it has remained elusive whether KimA is also controlled on the protein level. Much less is known about the potassium export systems of $B$. subtilis. The KhtSTU complex and the YugO proteins are suggested to be potassium exporters (Fujisawa et al., 2007; Prindle et al., 2015). In addition, the YjbQ protein is similar to the $S$. aureus potassium exporter CpaA, which was also shown to bind c-di-AMP via its RCK_C domain (Chin et al., 2015).

In general, RCK_C and CBS domains appear to contain conserved c-di-AMP binding sites (Corrigan et al., 2013; Sureka et al., 2014; Chin et al., 2015; Schuster et al., 2016; Huynh et al., 2016). B. subtilis encodes five proteins containing an RCK_C domain and 16 proteins with CBS domains (Zhu and Stülke, 2018), many of which are potential potassium and osmolyte transporters, respectively. To get a more comprehensive understanding of c-di-AMP-mediated signaling in this bacterium, we have tested the potential binding of the second messenger to these proteins as well as to the newly discovered potassium importer KimA. Our results support the idea that c-di-AMP is responsible for the global control of potassium homeostasis, by controlling both its uptake and export.

\section{RESULTS}

\section{Isolation of suppressor mutants that allow growth of a strain lacking c-di-AMP at $20 \mathrm{mM} \mathrm{KCl}$}

A B. subtilis strain lacking all three diadenylate cyclases is unable to grow at potassium concentrations of $5 \mathrm{mM}$ or higher. However, the acquisition of suppressor mutations at $5 \mathrm{mM}$ potassium that affect the cation exporter NhaK and result in enhanced potassium secretion prevents the toxic effect of potassium (Gundlach et al., 2017b). Yet, those suppressor mutants were not viable at a potassium concentration of $20 \mathrm{mM}$. To identify the growth-limiting problem, we sought to isolate suppressor mutants that tolerate this potassium concentration. For this purpose, we plated the c-di-AMP-free strain GP2222 (Gundlach et al., 2017b). on MSSM medium containing $20 \mathrm{mM} \mathrm{KCl}$. After four days at $37^{\circ} \mathrm{C}$, two colonies could be isolated. Both strains (GP2737 and GP2738) were subjected to whole genome sequencing to identify the responsible mutations. Both strains carried point mutations affecting the potassium transporter KimA (Trp-520 replaced by Gly) and KtrCD's regulatory subunit KtrC (Ala-73 replaced by Val). Moreover, both strains carried a point mutation in FlhA, which is part of the flagellar type III export apparatus. Finally, one of the mutants (GP2737) carried a frameshift mutation in odhA, which results in loss of 2-oxoglutarate dehydrogenase activity as indicated by the reddish colour of the colonies (Gundlach et al., 2017a).

To study the role of the mutations affecting the potassium uptake systems, we first attempted to delete the kimA and $k \operatorname{trC}$ genes in the c-di-AMP-free strain GP2222. While a control experiment (deletion of the nhak gene) was successful, the deletion of kimA or $k \operatorname{trC}$ was not possible. This 
observation suggests that the point mutations did not completely prevent the KimA and KtrC activities. The mutation in KtrC affects a highly conserved residue in the RCK_N domain. Similarly, the Trp-520 residue is conserved in the KimA proteins of B.subtilis, L.monocytogenes, S. aureus, and Mycobacterium tuberculosis. The conservation of the substituted amino acids suggests that the mutant proteins have reduced activity.

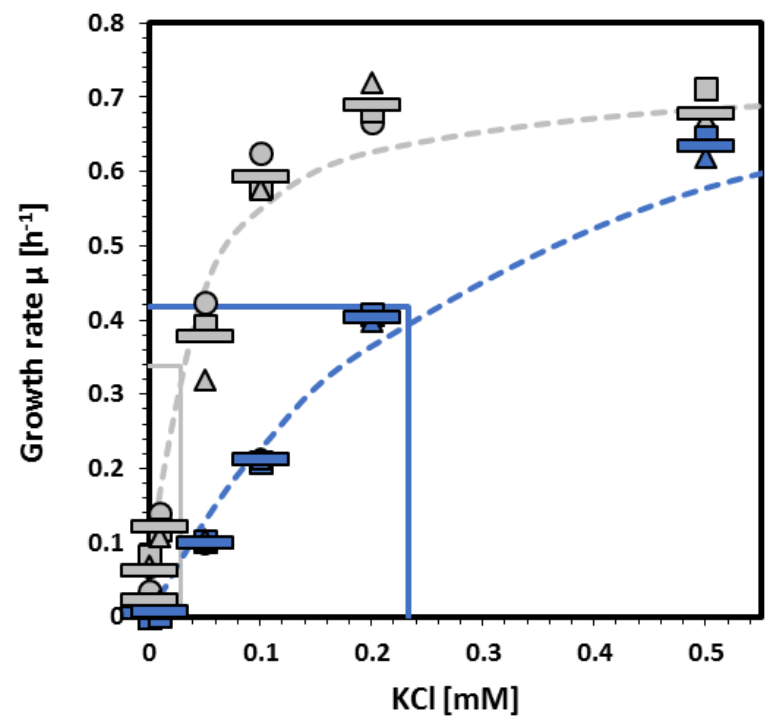

Fig. 3.1. Activity of KimA variant proteins in a heterologous comple- mentation assay. E. coli LB2003 was transformed with pGP2913 (WT KimA, gray) and pGP2993 (KimA-W520G, blue), respectively, and growth at different potassium concentrations was assessed over $24 \mathrm{~h}$. The growth rate $\mu$ was determined and plotted against the potassium concentrations. Dashed lines show the ideal progression of $\mu$ over the different potassium concentrations according to the Monod equation, and continuous lines show the determined Ks. Circles, squares, and triangles represent independent biological replicates. The bars indicate the means of the replicates.

Because no detailed information on the activity of KimA is available, we decided to analyze the activity of the mutant protein in a heterologous complementation assay using E. coli LB2003 (Stumpe and Bakker, 1997). This strain is deficient in the three major endogenous potassium uptake systems Trk, Kup, and Kdp, and is therefore not able to grow at low potassium concentrations without complementation using a gene encoding a potassium transporter. For the IPTG-inducible expression of the wild type and mutant KimA variants, we used plasmids pGP2913 and pGP2993, respectively. Accordingly, E. coli LB2003 was transformed with these plasmids or the empty vector control pWH844 (Schirmer et al., 1997) and growth was assessed in minimal medium supplemented with increasing KCl concentrations $(0.001,0.01,0.02,0.04,0.06,0.1,0.2,0.5,10$ and $50 \mathrm{mM} \mathrm{KCl}$ ) (see Fig. 3.1). While $50 \mathrm{mM} \mathrm{KCl}$ were required for growth of the strain carrying the empty vector, expression of wild type KimA (Gundlach et al., 2017b, this study) as well as of the KimA variant W520G allowed growth at much 
lower $\mathrm{KCl}$ concentrations. The determination of the growth rates at different potassium concentrations allowed fitting according to the Monod equation, an equation describing the growth of cultures, which is based on the Michaelis-Menten equation (Monod, 1949). This revealed the maximum specific growth rate $\mu_{\max }\left[\mathrm{h}^{-1}\right]$ and the substrate concentration that supports the half-maximal growth rate $\mathrm{K}_{\mathrm{s}}$ $[\mathrm{mM} \mathrm{KCl}]$ of the wild type and mutant KimA proteins. The mean $\mathrm{K}_{\mathrm{s}}$ values of three independent biological replicates are as follows: Wild type KimA had a $\mathrm{K}_{\mathrm{s}}$ of $0.029 \pm 0.0039 \mathrm{mM}$ for potassium, and a $\mu_{\max }$ of $0.68 \pm 0.005 \mathrm{~h}^{-1}$. In contrast, the mutant KimA protein had an apparent $K_{s}$ for potassium of $0.233 \pm 0.021 \mathrm{mM}$ and $a \mu_{\max }$ of $0.84 \pm 0.038 \mathrm{~h}^{-1}$. This eightfold reduction of the apparent affinity for potassium perfectly fits with the conclusion that the suppressor mutant is impaired in potassium uptake without completely preventing it.

\section{Analysis of a possible interaction between KimA and the PII-like c-di-AMP-binding protein DarA}

Often, transporters for molecules that may be toxic upon accumulation are controlled at the levels of expression and of transporter activity to avoid intoxication of the cells. This has been described for the ammonium transporter AmtB, which is only expressed under conditions of ammonium limitation and additionally inhibited by binding of the PII protein GInK if the ammonium concentrations suddenly increase (Coutts, 2002; Detsch and Stülke, 2003). The expression of the high-affinity potassium transporters KimA and KtrAB is controlled by a c-di-AMP-responsive riboswitch (Nelson et al., 2013; Gundlach et al., 2017b). In addition, KtrAB is inhibited upon binding of c-di-AMP, the second messenger that transduces the information on potassium accumulation (Corrigan et al., 2013; Gundlach et al., 2017b). For the novel potassium transporter KimA, it is not known, whether and how the activity of this protein is controlled. Following the concept of sustained sensing, we considered the possibility that the activity of KimA might be controlled by the PII-like c-di-AMP-binding protein DarA (Gundlach et al., 2015a), since KimA does not contain one of the known c-di-AMP-binding domains. To test this hypothesis, we first analyzed the localization of the DarA protein. The PII protein GlnK is found in the cytoplasm at low ammonium concentrations, but is recruited to the membrane via AmtB under conditions of ammonium excess (Detsch and Stülke, 2003). To study, whether the localization of DarA depends on potassium availability, we raised antibodies against the protein to facilitate its detection. B. subtilis was cultivated in MSSM minimal medium containing 0.1 or $5 \mathrm{mM}$ of $\mathrm{KCl}$, and the proteins were separated into cytoplasmic and membrane-bound fractions. The fractions were then assayed for the presence of DarA. As controls served the cytoplasmic protein CggR and the membrane protein RNase $Y$ (Meinken et al., 2003; Lehnik-Habrink et al., 2011). While the localization of the control proteins was observed as expected, we found DarA to be present exclusively in the cytoplasm (Fig. 3.2). This result suggests that DarA does not bind to the potassium transporter KimA to control its 
activity. To confirm this observation, we determined the cellular potassium concentrations in the wild type strain B. subtilis 168 and the isogenic darA mutant GP1712 (Gundlach et al., 2015a) by inductively coupled plasma optical emission spectrometry. Both strains had very similar intracellular potassium concentrations of $5.1 \pm 0.4$ and $4.8 \pm 0.3 \mu \mathrm{g} \mathrm{K}^{+} \mathrm{ml}^{-1} \mathrm{OD}_{600^{-1}}$, respectively. Taken together, these results strongly suggest that the activity of KimA is not controlled by the c-di-AMP-binding protein DarA.

Table 3.1 Expression plasmids used in the DRaCALA assay.

\begin{tabular}{|c|c|c|c|}
\hline Plasmid & Protein & Function $^{1}$ & Domain \\
\hline pGP2594 & KtrA & Peripheral membrane component $\mathrm{K}^{+}$transporter & RCK_C \\
\hline pGP2906 & KhtT & $\mathrm{K}^{+} / \mathrm{H}^{+}$antiporter & RCK_C \\
\hline pGP2907 & KtrC & Peripheral membrane component $\mathrm{K}^{+}$transporter & RCK_C \\
\hline pGP2908 & YrvC & Unknown & RCK_C \\
\hline pGP2908 & YjbQ/ CpaA & Unknown & RCK_C \\
\hline pGP2913 & KimA & $\mathrm{K}^{+}$transporter & 2 \\
\hline pGP2922 & AcuB & Unknown & CBS \\
\hline pGP2923 & $\mathrm{CcpN}$ & Transcriptional repressor & CBS \\
\hline pGP2924 & OpuAA & Glycine betaine $A B C$ transporter (ATP-binding protein) & CBS \\
\hline pGP2927 & MgtE & Primary $\mathrm{Mg}^{2+}$ transporter & CBS \\
\hline pGP2928 & YhdT & Unknown & CBS \\
\hline pGP2929 & YkuL/ DarB & Unknown & CBS \\
\hline pGP2930 & YhcV & Unknown, forespore-specific sporulation protein & CBS \\
\hline pGP2931 & YhdP & Potential $\mathrm{Mg}^{2+}$ efflux pump & CBS \\
\hline pGP2932 & YrkA & Unknown & CBS \\
\hline pGP2933 & Ytol & Unknown, similar to transcriptional regulator (GntR family) & CBS \\
\hline pGP2934 & YlbB & Unknown, putative oxidoreductase & CBS \\
\hline pGP2935 & YqhB & General stress protein & CBS \\
\hline pGP2936 & Yugs & Unknown & CBS \\
\hline pGP2937 & GuaB & Biosynthesis of GMP & CBS \\
\hline pGP2938 & OpuBA & Choline $A B C$ transporter (ATP-binding protein) & CBS \\
\hline pGP2939 & OpuCA & $\begin{array}{l}\text { Glycine betaine/ carnitine/ choline } A B C \text { transporter (ABC- } \\
\text { binding protein) }\end{array}$ & CBS \\
\hline
\end{tabular}

${ }^{1}$ Protein functions have been retrieved from the SubtiWiki database (Zhu and Stülke, 2018).

${ }^{2}$ For KimA, no conserved c-di-AMP-binding domain has been identified so far.

\section{Identification of c-di-AMP target proteins}

Because our localization studies did not support the idea that the activity of KimA is controlled by DarA, we considered the possibility that c-di-AMP might directly bind and control KimA. Additionally, we aimed at identifying further targets that directly bind c-di-AMP in B. subtilis to detail our understanding of its regulatory function. For this broader approach we focused on the five proteins containing RCK_C 
domains and the 16 proteins containing CBS domains encoded in B. subtilis (Zhu and Stülke, 2018, see Table 3.1). Interestingly, many of these proteins are implicated in ion or compatible solute transport.

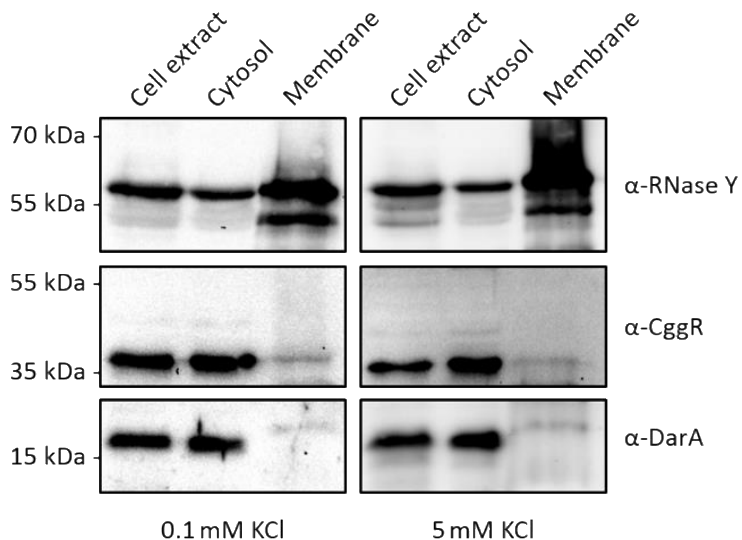

Fig. 3.2. The c-di-AMP-binding protein DarA is a cytoplasmic protein. B. subtilis 168 was cultivated in MSSM minimal medium containing 0.1 or $5 \mathrm{mM} \mathrm{KCl}$. Crude extracts were separated by ultracentrifugation to obtain cytosolic and membrane fractions. The presence of DarA was tested using antibodies recognizing DarA. To check for successful separation, the cytosolic and membrane fraction were tested with the specific antibodies recognizing CggR and Rny, respectively.

We used $E$. coli cells as expression system since c-di-AMP is not synthesized by this bacterium. The 22 selected genes were cloned into the expression vector pWH844 (Schirmer et al., 1997). After checking expression of all genes, using strain E. coli BL21 as a host, the lysates of strains carrying the corresponding plasmids were assessed for a possible interaction with c-di-AMP in vitro using the differential radial capillary action of ligand assay (DRaCALA) (Roelofs et al., 2011, see Experimental procedures).

In an initial screen for c-di-AMP binding, eight target proteins could be identified (Fig $3.3 \mathrm{a}, \mathrm{b}$ ). Among these proteins, the known c-di-AMP-binding protein KtrA served as positive control (Corrigan et al., 2013; Rocha et al., 2019). In addition to KtrA, three other proteins containing an RCK_C domain were identified as c-di-AMP-binding proteins. KtrC, the regulatory subunit of the low affinity potassium transporter KtrCD, the cytoplasmic subunit KhtT of the $\mathrm{K}^{+} / \mathrm{H}^{+}$antiporter KhtSTU and YjbQ, a putative cation exporter, showed strong binding to c-di-AMP. Only YrvC, a protein of unknown function, showed non-significant binding. Of the proteins containing a CBS domain, the $\mathrm{Mg}^{2+}$ transporter MgtE, the ATPbinding protein OpuCA of the compatible solute transporter OpuC and YkuL, a protein of unknown function, showed increased binding affinity towards the di-nucleotide (Fig. 3.3a). Importantly, the novel potassium transporter KimA, which possesses none of the conserved binding motifs, also bound c-di-AMP (Fig. 3.3b). Strikingly, among the c-di-AMP-binding proteins identified in this screen, YkuL is the only c-di-AMP receptor protein that is not associated to the cell membrane. Binding of OpuCA and 
YkuL to c-di-AMP is in excellent agreement with previously published results (Sureka et al., 2014; Schuster et al., 2016; Huynh et al., 2016). Because YkuL is composed of two reiterated CBS domains and thus seems to have a regulatory function, we refer to this protein as DarB (cprotein $\underline{B}$, in analogy to DarA) (Gundlach et al., 2015a). The specificity of c-di-AMP binding to the identified proteins was confirmed by competition experiments with cold ATP or c-di-AMP from overnight grown cultures (Fig. 3.3c, d). For all tested proteins, ATP was unable to block c-di-AMP binding, while cold c-di-AMP outcompeted the radioactively labeled c-di-AMP. This demonstrates the specificity of c-di-AMP binding and indicates that these proteins are true targets of the second messenger.

A

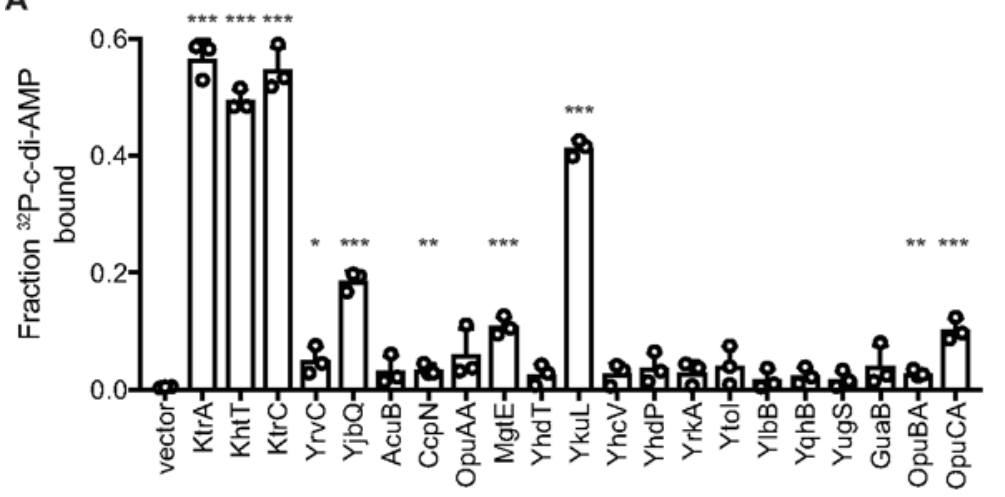

B

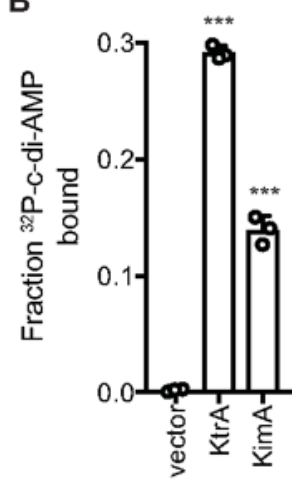

C

$\square$ ATP

c-di-AMP
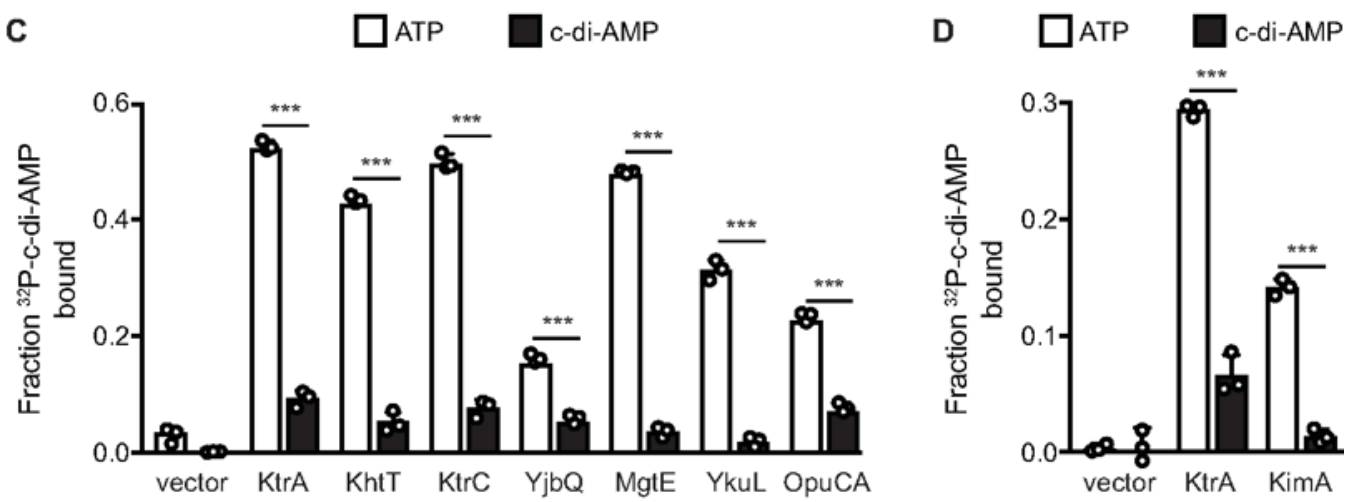

Fig. 3.3. A subset of c-di-AMP-binding proteins overexpressed in E. coli whole cell lysates determined by DRaCALA. A and B, fraction bound of radiolabeled [ $\left.{ }^{32} \mathrm{P}\right] \mathrm{c}$-di-AMP is shown for lysates from E. coli induced for the expression ofthe indicated genes in presence of nonspecific ATP competitor at $100 \mu \mathrm{M}$ (A) or $200 \mu \mathrm{M}$ (B). C and $\mathrm{D}$, induced lysates overexpressing the indicated gene are tested for specificity ofc-di-AMP binding to $\left.{ }^{32} \mathrm{P}\right] \mathrm{c}$-diAMP by competition assays. C, competitors are $100 \mu \mathrm{M}$ ATP (open bars) or $100 \mu \mathrm{M}$ ATP and $100 \mu \mathrm{M}$ c-di-AMP (closed bars). D, competitors are $200 \mu \mathrm{M}$ ATP (open bars) or $200 \mu \mathrm{M}$ ATP and $100 \mu \mathrm{M}$ c-di-AMP (closed bars). Significance of binding was determined using unpaired $t$ test between vector-only control and indicated gene ( $A$ and B) or between ATP and c-di-AMP competitors ( $C$ and D). $p$ values of $<0.05,<0.01$, and $<0.001$ are indicated by $* * *$, and $* * *$, respectively. 
In total, we identified six novel c-di-AMP receptor proteins in B. subtilis. These novel target proteins are involved in potassium uptake and export, but also in magnesium and osmolyte uptake. The fact that c-di-AMP does not bind specifically to proteins involved in one homeostatic process suggests that control of potassium homeostasis is not the only function of the essential second messenger (Zeden et al., 2018).

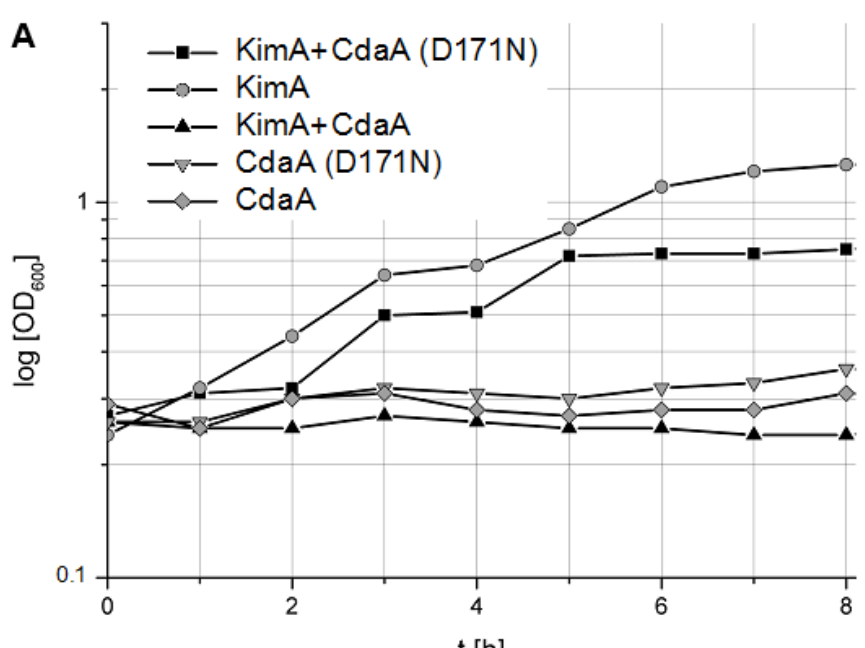

$\mathrm{t}[\mathrm{h}]$

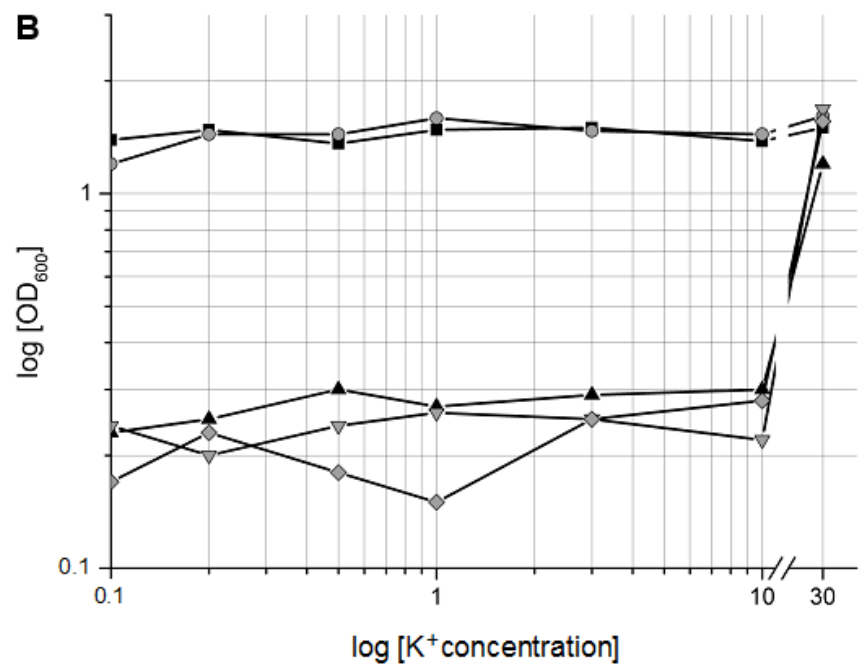

Fig. 3.4. CdaA-dependent inhibition of KimA-mediated growth. (A) growth curves of $E$. coli LB2003 expressing KimA and/or variants of CdaA in $\mathrm{K}$ minimal medium containing $3 \mathrm{mM} \mathrm{KCl}$. (B) A600 of E. coli LB2003 expressing KimA and/or variants of $\mathrm{CdaA}$ grown for $24 \mathrm{~h}$ in minimal medium with potassium concentrations ranging from 0.1 to $30 \mathrm{mM}$.

\section{c-di-AMP inhibits potassium transport by KimA}

The specific interaction of KimA with c-di-AMP suggested a functional role for c-di-AMP in the control of KimA activity. Therefore, we analyzed the impact of this second messenger nucleotide on the potassium transport activity of KimA by a growth complementation assay. For this purpose, a co- 
expression system producing the c-di-AMP-synthesizing diadenylate cyclase CdaA and the potassium transporter KimA was established in the potassium transporter-deficient mutant E. coli LB2003 (Stumpe and Bakker, 1997). Importantly, E. coli lacks c-di-AMP synthesizing enzymes and is unable to produce this second messenger (Corrigan et al., 2011; Mehne et al., 2014; Rosenberg et al., 2015). The growth phenotype upon co-expression of $c d a A$ from L. monocytogenes, $\left(\mathrm{CdaA}^{\mathrm{Lmo}}\right)$ and $\operatorname{kimA}$ genes reflects the effect of c-di-AMP binding on KimA. The co-expression of KimA with an inactive diadenylate cyclase, $\mathrm{CdaA}^{\mathrm{Lmo}}(\mathrm{D} 171 \mathrm{~N})$ (Rosenberg et al., 2015), served as a negative control. Similarly, the growth phenotype of LB2003 expressing kimA and cdaA alone were analyzed.

Growth curves of LB2003 transformed with the respective plasmids and induced with $0.002 \%$ arabinose were recorded at $0.1,0.2,0.5,1,3,10$ and $30 \mathrm{mM} \mathrm{KCl}$ in phosphate-buffered minimal medium for $24 \mathrm{~h}$ (Fig. 3.4). LB2003 harboring pBP370 (CdaA ${ }^{\mathrm{Lmo}}$ ) and pBP373 (CdaA ${ }^{\mathrm{Lmo*}}$ ) (Quintana et al., 2019) only grew at $30 \mathrm{mM} \mathrm{KCl}$, while LB2003 producing KimA (plasmid pB24C3H-KimA) showed growth complementation at all tested potassium concentrations. KimA thus facilitates the uptake of potassium, while the expression of $c d a A$ has no general inhibitory effect, which is in agreement with previous observations (Gundlach et al., 2017b; Quintana et al., 2019). A very similar growth behavior to LB2003 expressing kimA was observed upon co-production of KimA (pB24C3H-KimA) and the inactive $\mathrm{CdaA}^{\mathrm{Lmo*}}$ (pBP373). However, the co-expression KimA (pB24C3H-KimA) with active CdaA ${ }^{\mathrm{Lmo}}$ (pBP370) abolished cell growth below $30 \mathrm{mM} \mathrm{KCl}$. Thus, we can conclude that the binding of c-di-AMP produced by $\mathrm{CdaA}^{\mathrm{Lmo}}$ to KimA inhibits its potassium transport activity.

\section{DISCUSSION}

The second messenger c-di-AMP is essential in many bacteria, including the Gram-positive model organism B. subtilis and the closely related pathogenic bacteria L. monocytogenes and S. aureus. Previous pull-down experiments revealed several target proteins for the two pathogenic bacteria (Corrigan et al., 2013; Sureka et al., 2014), but for B. subtilis the unknown c-di-AMP receptor DarA and the potassium channel subunits KtrA and $\mathrm{KtrC}$ were the only target proteins that had been identified (Gundlach et al., 2015b; Rocha et al., 2019). None of the identified c-di-AMP target proteins was essential for cell viability and therefore the essential function of the second messenger has long remained elusive (Commichau et al., 2015).

In this study, we tested c-di-AMP binding for the recently discovered potassium transporter KimA as well as for proteins containing specific domains, either the RCK_C or CBS domain. These protein domains have been shown to bind directly to c-di-AMP (Sureka et al., 2014). Of the 22 tested proteins, we identified six novel c-di-AMP targets, i. e. the potassium transporter KimA, the RCK_C subunit (KhtT) of a potassium/ proton antiporter KhtSTU (Fujisawa et al., 2007), the primary 
magnesium importer MgtE (Wakeman et al., 2014), the glycine-betaine transporter subunit OpuCA (Kappes et al., 1999) and the two proteins of unknown function DarB and YjbQ. Binding of c-di-AMP to DarB, OpuCA, and YjbQ homologs has been reported previously for L. monocytogenes (Sureka et al., 2014), whereas KimA, KhtT, and MgtE have not been previously identified as c-di-AMP targets. The common denominator between KimA, KhtT, YjbQ, MgtE and OpuCA is that all five proteins are transmembrane proteins. The control of potassium import has been previously linked to the essentiality of c-di-AMP in B. subtilis (Gundlach et al., 2017b). The here identified c-di-AMP-binding potassium export systems Kht and YjbQ suggest that c-di-AMP plays a more general role in controlling potassium uptake and release. Moreover, it is tempting to speculate that the control of magnesium and osmolyte homeostasis are additional essential functions of c-di-AMP. Indeed, an implication of c-di-AMP signaling in osmoregulation is a common theme in many bacteria (see Commichau et al. 2018a for review). In L. monocytogenes, the essentiality of the second messenger is also not linked to the control of one specific protein, but to the general control of the stringent response factor (p)ppGpp (Whiteley et al., 2015).

Importantly, this study identified the potassium transporter KimA as an additional target of c-di-AMP. As potassium is both essential and toxic for the cells (Radchenko et al., 2006; Gundlach et al., 2017b), the control of its homeostasis is of utmost importance for the bacteria. A strain lacking c-di-AMP can grow at elevated potassium concentrations only, if potassium ions can be more efficiently exported (Gundlach et al., 2017b) or if the uptake of potassium is reduced by mutations affecting conserved residues in the transporters KtrC and KimA (this work). This observation already suggests that the KimA activity is reduced in the presence of c-di-AMP, and that a corresponding reduction must be achieved in the absence of c-di-AMP by the acquisit ion of a mutation. As the mechanism of KimA control by c-di-AMP had been unknown prior to this study, we hypothesized that the KimA activity might be controlled by the c-di-AMP-binding PII-like protein DarA, in analogy to the control of the ammonium transporter AmtB by the PII protein GlnK (Coutts, 2002; Detsch and Stülke, 2003). However, our experiments did not support this possibility, and suggested that KimA might be subject to direct control by c-di-AMP. This idea was confirmed by the detection of binding of c-di-AMP to KimA and by the observation that this interaction inhibits the potassium uptake activity of KimA.

KimA represents a completely novel group of c-di-AMP-binding proteins that does not contain any of the previously identified domains that bind the second messenger. Moreover, KimA is the prototype of the fourth class of proteins that are involved in the control of potassium homeostasis and that are controlled by c-di-AMP in Gram-positive bacteria. So far, the potassium transporters containing an RCK_C domain have been studied in several bacteria, and we have recently identified the KupA and KupB proteins as novel targets of c-di-AMP in L. lactis (Quintana et al., 2019). In all cases, 
these potassium transporters are inhibited by c-di-AMP. Interestingly, a L. lactis strain that accumulates c-di-AMP was extremely sensitive to salt stress, but the acquisition of a mutation in KupB restored growth. In this case, the variant KupB protein had an increased activity to compensate for the strong inhibition upon c-di-AMP accumulation (Quintana et al., 2019). Thus, accumulation of c-di-AMP had just the opposite effect for the mutational adaptation of the potassium transporter as observed here for lack of c-di-AMP. KimA adds a third protein family to the list of c-di-AMP inhibited potassium transporters.

Interestingly, c-di-AMP does not only bind to proteins involved in potassium uptake but also to a riboswitch that controls the expression of the high-affinity KtrAB and KimA transporters in B. subtilis. Binding of c-di-AMP to the riboswitch prevents expression beyond the riboswitch and does thus inhibit expression of the transporter genes (Nelson et al., 2013; Gundlach et al., 2017b). Moreover, c-di-AMP binds to the sensor kinase $\mathrm{KdpD}$, which is required for the expression of the high affinity potassium transport system KdpFABC in S. aureus. Again, binding of c-di-AMP inhibits the activity of the protein and does thus prevent expression of the transporter (Moscoso et al., 2016). In conclusion, c-di-AMP governs potassium uptake by direct binding to and inhibition of potassium uptake systems and by controlling the expression of the high-affinity transporters.

In addition to KimA, we have identified the KhtT and YjbQ proteins as novel targets of c-di-AMP. While KhtT has been demonstrated to be a subunit of a potassium/ proton antiporter in B. subtilis (Fujisawa et al., 2007), less is known on YjbQ. The corresponding S. aureus protein CpaA (cation/ proton antiporter A) has also been shown to bind c-di-AMP (Corrigan et al., 2013). This protein is a potassium exporter, and interestingly, its activity is stimulated by c-di-AMP (Chin et al., 2015). Based on the $52 \%$ amino acid identity between the proteins from both organisms and on the common regulation by c-di-AMP, we rename $B$. subtilis YjbQ to CpaA. It is interesting to mention that in screens for c-di-AMP-free strains that are viable at increased potassium concentrations, we never found mutations that affected the potassium exporters KhtSTU or CpaA. In contrast, variants of the NhaK cation exporter with increased specificity for potassium were selected at $5 \mathrm{mM}$ potassium (Gundlach et al., 2017b) whereas mutations resulting in reduced potassium uptake were found at $20 \mathrm{mM}$ potassium (this work). These findings support the idea that the potassium exporters are inactive unless they bind and become activated by c-di-AMP. Obviously, mutations that overcome this requirement are rather unlikely.

Taken together, the results from our suppressor screens, the biochemical investigation of S. aureus $\mathrm{CpaA}$ as well as the physiological logics all converge to support the idea that c-di-AMP inhibits potassium uptake at the levels of transporter expression and activity, and stimulates potassium export by activating the potassium/ proton antiporters (see Fig. 3.5). This extends the idea that the control of 
potassium homeostasis by controlling potassium uptake is an essential function of c-di-AMP to the export of this cation. The binding of multiple potassium transporters as well as the dual control of both potassium transporter expression and activity as observed for KtrAB and KimA (Corrigan et al., 2013; Nelson et al., 2013; Gundlach et al. 2017b; Rocha et al. 2019b, this work) makes c-di-AMP-mediated signaling on potassium homeostasis a paradigm for the concept of sustained sensing (Orr et al., 2016). It is amazing that different classes of (functionally different) proteins and even of a riboswitch have evolved to be controlled by a single second messenger, c-di-AMP! Thus, c-di-AMP is the major effector of potassium uptake in the firmicutes. Further work will be required to understand the roles of the distinct potassium export systems and to unravel the functions of the so far uncharacterized c-di-AMPbinding proteins, DarA and DarB.

A

Wild type, high potassium Concentration

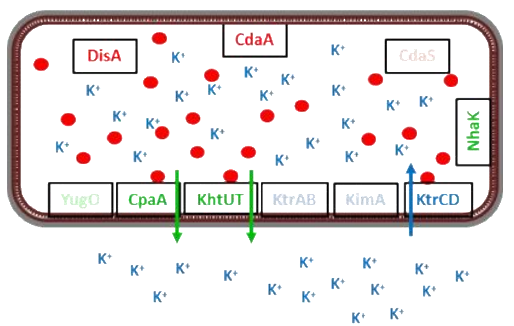

B

Wild type, low potassium concentration

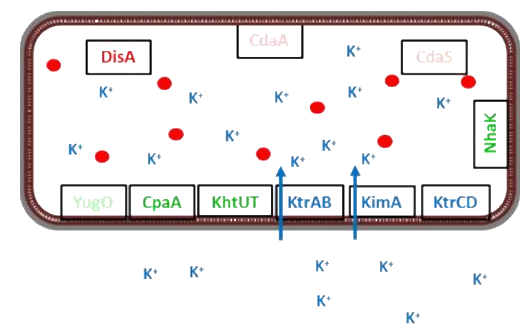

C

c-di-AMP-free strain, high potassium concentration

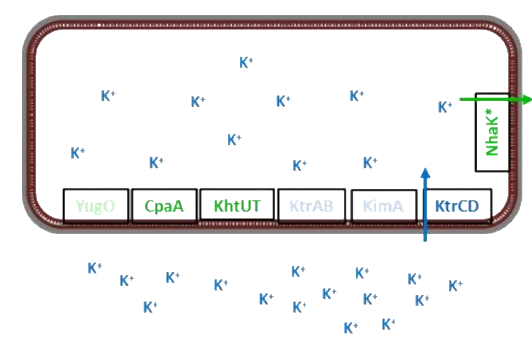

Fig. 3.5. Control of potassium uptake and export in B. subtilis by c-di-AMP. (A) in the WT strain, the potassium exporters CpaA and KhtUT, as well as the low-affinity transporter KtrCD, are expressed at high potassium concentrations. Potassium is taken up by KtrCD and triggers the production of c-di-AMP by CdaA and DisA. Upon accumulation of potassium and the second messenger, c-di-AMP binds to KtrC to prevent further potassium uptake. Simultaneous binding of c-di-AMP to CpaA and KhtUT triggers potassium export. Thus, the cell prevents potassium intoxication. (B) at low potassium concentrations, all three uptake systems as well as the two exporters are expressed. Potassium is transported by the high-affinity transporters KtrAB and KimA. The low intracellular potassium concentration results only in a very limited synthesis of c-di-AMP that is not sufficient to inhibit or activate potassium uptake or export, respectively. (C) a strain lacking C-di-AMP is unable to grow at 
high potassium concentrations because ofthe unlimited influx of the ion via KtrCD. The potassium exporters are inactive in the absence of the second messenger. Therefore, growth of this strain in the presence of potassium requires a novel active potassium exporter. Under these conditions, the bacteria acquire mutations affecting the NhaK cation/proton antiporter that result in increased potassium export (Gundlach et al., 2017b).

\section{EXPERIMENTAL PROCEDURES}

\section{Strains, media and growth conditions}

E. coli DH5 $\alpha$ (Sambrook and Russell, 2001) was used for cloning and for the expression of recombinant proteins. E. coli LB2003 (Stumpe and Bakker, 1997) was used to assay potassium transporter activity. All B. subtilis strains used in this study are derivatives of the laboratory strain 168 . B. subtilis was grown in Luria-Bertani (LB) or in sporulation (SP) medium (Sambrook and Russell, 2001; Kunst and Rapoport, 1995). E. coli was cultivated in MSSM medium (Gundlach et al., 2017b) or in modified M9 medium in which $\mathrm{KH}_{2} \mathrm{PO}_{4}$ was replaced by $\mathrm{NaH}_{2} \mathrm{PO}_{4}$ and $50 \mathrm{mM} \mathrm{KCl}$ was added. The media were supplemented with ampicillin $(100 \mu \mathrm{g} / \mathrm{ml})$, kanamycin $(10$ and $50 \mu \mathrm{g} / \mathrm{ml}$ for B. subtilis and E. coli, respectively), chloramphenicol $(5 \mu \mathrm{g} / \mathrm{ml})$, tetracyclin $(12.5 \mu \mathrm{g} / \mathrm{ml})$, spectinomycin $(150 \mu \mathrm{g} / \mathrm{ml})$ or erythromycin and lincomycin ( 2 and $25 \mu \mathrm{g} / \mathrm{ml}$, respectively) if required.

\section{DNA manipulation and genome sequencing}

Transformation of E. coli and plasmid DNA extraction were performed using standard procedures (Sambrook and Russell, 2001). All commercially available plasmids, restriction enzymes, T4 DNA ligase and DNA polymerases were used as recommended by the manufacturers. Chromosomal DNA of B. subtilis was isolated as described (Kunst and Rapoport, 1995). B. subtilis was transformed with plasmid and genomic DNA according to the two-step protocol (Kunst and Rapoport, 1995).

To identify the mutations in the suppressor mutant strains GP2737 and GP2738, the genomic DNA was subjected to whole-genome sequencing (Reuß et al., 2017). Briefly, the reads were mapped on the reference genome of B. subtilis 168 (GenBank accession number: NC_000964) (Barbe et al., 2009). Mapping of the reads was performed using the Geneious software package (Biomatters Ltd., New Zealand) (Kearse et al., 2012). Single nucleotide polymorphisms were considered as significant when the total coverage depth exceeded 25 reads with a variant frequency of $\geq 90 \%$. All identified mutations were verified by PCR amplification and Sanger sequencing.

\section{Plasmid constructions}

The selected genes were amplified using chromosomal DNA of B. subtilis 168 as the template and appropriate nucleotides that attached BamHI (or BgIII) and Sall restriction sites to the fragments and cloned between the BamHI and Sall sites of the expression vector pWH844 (Schirmer et al., 1997; see 
Table 3.1). The mutant kimA allele encoding KimA (W520G) was amplified using the oligonucleotides JN465 and JN466 (Gundlach et al., 2017b) and cloned between the BamHI and Pstl sites of pWH844. The resulting plasmid was pGP2993. The construct of KimA used in the co-expression of KimA and diadenylate cyclases was cloned by restriction-free cloning into a modified pBAD24 vector that bears a His-tag in the C-terminus, resulting in the plasmid pB24C3H-KimA.

\section{Identification of c-di-AMP-binding proteins by Differential radial capillary action of ligand assay (DRaCALA)}

Expression of the genes upon induction using $1 \mathrm{mM}$ of IPTG was verified by analyzing the protein patterns of the expression strains by SDS-PAGE. ${ }^{32}$ P-labeled c-di-AMP synthesis was performed using purified diadenylate cyclase DisA (Corrigan et al., 2013). The analysis of protein-ligand interaction was performed using $E$. coli whole cell lysates that were grown to an $\mathrm{OD}_{600}$ of $0.5-1.0$ and induced for 4 hours by 1 mM IPTG as described (Roelofs et al., 2011; Orr et al., 2016) or grown overnight in the presence of $50 \mu \mathrm{g} / \mathrm{ml}$ carbenicillin and $1 \mathrm{mM}$ IPTG. All binding reactions were performed in $1 \mathrm{x}$ binding buffer (10 mM Tris, pH 8.0, $100 \mathrm{mM} \mathrm{NaCl}, 5 \mathrm{mM} \mathrm{MgCl} 2$ ) containing $10 \mathrm{pM}{ }^{32} \mathrm{P}$-c-di-AMP. Proteinligand mixtures were spotted on nitrocellulose membrane (Amersham Hybond-ECL; GE Healthcare) and allowed to dry. The areas and intensities of spots were quantified by exposing phosphorimager screens and scanning by FUJI FLA-7000 phosphorimager. The competition assays were performed with indicated concentrations of unlabeled ATP and c-di-AMP (Axxora).

\section{Preparation of membrane fractions}

Cultures of $B$. subtilis were harvested by centrifugation $\left(4,400 \times \mathrm{g}, 10 \mathrm{~min}, 4^{\circ} \mathrm{C}\right)$. The following steps were done as described previously (Gundlach et al., 2015b). Briefly, the cells were lysed by sonication, the cellular debris removed, and the fractions of the cell extract were separated by ultracentrifugation. The membrane pellet was washed for three times and finally resuspended in phosphate buffer ( $50 \mathrm{mM}$ $\mathrm{Na}_{2} \mathrm{HPO}_{4}, 50 \mathrm{mM} \mathrm{NaH} \mathrm{PO}_{4}, \mathrm{pH} 6.8$ ). To assess the quality of the preparations, the fractions were analyzed for the presence of $\mathrm{CggR}$ and $\mathrm{RNase} \mathrm{Y}$ using polyclonal rabbit antibodies raised against these proteins (Meinken et al., 2003; Zweers et al., 2009).

\section{Western blot analysis}

The DarA protein was purified using E. coli DH5 $\alpha$ carrying the expression vector pGP2601 as described previously (Gundlach et al., 2015b). Purified His 6 -DarA was used to generate rabbit polyclonal antibodies. For Western blot analysis, B. subtilis cell extracts were separated on $12.5 \%$ SDS polyacrylamide gels. After electrophoresis, the proteins were transferred to a polyvinylidene difluoride 
membrane (PVDF, BioRad) by electroblotting. The primary antibodies were visualised by using antirabbit IgG-AP secondary antibodies (Promega) and the CDP* detection system (Roche Diagnostics) as described previously (Lehnik-Habrink et al., 2011).

\section{Determination of cellular potassium pools}

The cellular potassium pools were determined as described previously (Gundlach et al., 2017a). Briefly, B. subtilis cells were cultivated in MSSM medium supplemented with $0.1 \mathrm{mM} \mathrm{KCl}$. Cells were pelleted, transferred onto ash-free filter discs, and dried. The dried filter disks were cut into small pieces and reduced into a fluid state through pressure and $2 \mathrm{ml}$ of $65 \% \mathrm{HNO}_{3}$ for 7 hours at $185^{\circ} \mathrm{C}$ in $25 \mathrm{ml} \mathrm{Teflon}$ beakers (PDS-6 Pressure Digestion System, Loftfield, Göttingen, Germany). After digestion, the fluid content was transferred into an Erlenmeyer flask and diluted with demineralised water to a volume of $50 \mathrm{ml}$. The total potassium content of the cells in this solution was determined by ICP-OES (Inductively coupled plasma optical emission spectrometry) analysis (Optima 5300 DV, Perkin Elmer, Waltham, Massachusetts, USA). Light emission at $766.49 \mathrm{~nm}$ that is indicative of the potassium concentration in the sample was recorded.

\section{Determination of specific growth parameters}

The growth characteristics of $E$. coli LB2003 complemented with plasmid-based KimA, KimA-W520G or empty vector were determined as follows. Potassium was used as the growth-limiting factor. The bacteria were inoculated in $\mathrm{LB}$ medium containing $50 \mathrm{mM} \mathrm{KCl}$ and precultured in MSSM medium, supplemented with thiamin $(1 \mathrm{mg} / \mathrm{ml})$ and $50 \mathrm{mM} \mathrm{KCl}$. The cultures were grown until exponential phase, harvested, and the cells were incubated for $1 \mathrm{~h}$ in potassium-free medium, and washed three times in 1 x MSSM buffer. Afterwards, the cells were adjusted to $\mathrm{OD}_{600} 1.0$ and used to inoculate a 96 well plate (Microtest Plate 96 Well, Sarstedt) containing MSSM medium adjusted to the required potassium concentrations and $50 \mu \mathrm{M}$ IPTG to induce expression of kimA. Growth was tracked in an Epoch 2 Microplate Spectrophotometer (BioTek Instruments) at $37^{\circ} \mathrm{C}$ with linear shaking at $237 \mathrm{cpm}$ $(4 \mathrm{~mm})$ for $20 \mathrm{~h}$, and optical density at $600 \mathrm{~nm}$ was measured in $10 \mathrm{~min}$ intervals. The exponential growth phase was used to determine the growth rate $\mu\left[h^{-1}\right]$. The growth rates were then plotted against the potassium concentrations. This allowed fitting to the Michaelis-Menten equation and calculation of $\mathrm{V}_{\max }\left[\mathrm{h}^{-1}\right]$ and the apparent $\mathrm{K}_{\mathrm{M}}[\mathrm{mM} \mathrm{KCl}]$ using the solver tool of Excel 2012 (Microsoft). Experiments were repeated with three biological replicates. 


\section{Co-expression of KimA and diadenylate cyclases under potassium limitation}

E. coli LB2003 was transformed with plasmids pB24C3H-KimA, pBP370 and pBP373 that encode KimA, $\mathrm{CdaA}$ and the inactive mutant of $\mathrm{CdaA}^{\mathrm{D} 171 \mathrm{~N}}$, alone and in combination. Cells were cultivated in $\mathrm{K}$ medium (34 mM Na $2 \mathrm{HPO}_{4}, 17 \mathrm{mM} \mathrm{NaH} \mathrm{PO}_{4}, 1 \mathrm{mM}$ trisodium-citrate, $\left.7.6 \mathrm{mM}\left(\mathrm{NH}_{4}\right)_{2} \mathrm{SO}_{4}\right)$ supplemented with $20 \mu \mathrm{g} / \mathrm{ml} \mathrm{L}-$ methionine, $6 \mu \mathrm{M}$ Fe(II)SO $\mathrm{S}_{4}, 1 \mu \mathrm{g} / \mathrm{ml}$ thiamine, $0.4 \mu \mathrm{M} \mathrm{Mg} \mathrm{SO}_{4}, 0.2 \%$ glycerol, $30 \mathrm{mM}$ potassium $\left(\mathrm{K} 30,12 \mathrm{mM} \mathrm{K}_{2} \mathrm{HPO}_{4}, 6 \mathrm{mM} \mathrm{KH}_{2} \mathrm{PO}_{4}\right.$ ), and the corresponding antibiotics. An overnight culture was harvested and washed two times in $\mathrm{K}$ medium (46 mM Na $2 \mathrm{HPO}_{4}, 23 \mathrm{mM}$ $\mathrm{NaH}_{2} \mathrm{PO}_{4}, 1 \mathrm{mM}$ trisodium-citrate, $\left.7.6 \mathrm{mM}\left(\mathrm{NH}_{4}\right)_{2} \mathrm{SO}_{4}\right)$ supplemented with $10 \mathrm{mM} \mathrm{KCl}$. These samples were then used to inoculate fresh $\mathrm{K}$ medium with the same supplements plus $0.002 \% \mathrm{~L}$-arabinose to induce protein expression and different concentrations of $\mathrm{KCl}$ varying from $0.1 \mathrm{mM}$ to $30 \mathrm{mM}$. Cultures were incubated at $37^{\circ} \mathrm{C}$ with continuous orbital shaking and $\mathrm{OD}_{600}$ measurements were taken every hour.

\section{ACKNOWLEDGMENTS}

We would like to thank Suzanna Grubek for the help with the cloning experiments. We are grateful to Johannes Gibhardt and Fabian Commichau for helpful discussions. This work was supported by grants from the Deutsche Forschungsgemeinschaft via Priority Program SPP1879 (to J.S.) and from the National Institute of Health (NIH, Al133670) (to V.T.L.).

\section{CONFLICT OF INTEREST}

The authors declare that they have no conflicts of interest with the contents of this article. 


\section{Chapter 4 - Regulation of potassium uptake by KtrCD}

The results described in this chapter were originally published in Journal of Bacteriology (doi: 10.1128/JB.00138-20):

\section{Two ways to convert a low- to a high-affinity potassium channel: Control of Bacillus subtilis KtrCD by glutamate}

Larissa Krüger ${ }^{1}$, Christina Herzberg ${ }^{1}$, Robert Warneke ${ }^{1}$, Anja Poehlein ${ }^{2}$, Janina Stautz ${ }^{3}$, Martin Wei $\aleph^{1}$, Rolf Daniel ${ }^{2}$, Inga Hänelt ${ }^{3}$, and Jörg Stülke ${ }^{1}$

\footnotetext{
${ }^{1}$ Department of General Microbiology, GZMB, Georg-August-University Göttingen, Germany

${ }^{2}$ Department of Genomic and Applied Microbiology, GZMB, Georg-August-University Göttingen, Germany

${ }^{3}$ Institute of Biochemistry, Goethe University Frankfurt, Germany
}

\section{AUTHOR CONTRIBUTIONS}

LK and JöS conceived the study and designed the experiments. LK, CH, RW, AP, MW, and RD carried out the experiments. LK and $\mathrm{CH}$ isolated the suppressors of the c-di-AMP-free strain in the presence of glutamate. LK and $\mathrm{CH}$ constructed the $B$. subtilis mutants and characterized the phenotypes. LK conducted the kinetic experiments in E. coli. RW introduced the mutation into ktrD. LK, JaS, IH and JöS wrote the manuscript. All authors approved the manuscript. IH and JöS acquired funding. IH and JöS provided supervision. 


\section{ABSTRACT}

Potassium and glutamate are the major cation and anion, respectively, in every living cell. Due to the high concentrations of both ions, the cytoplasm of all cells can be regarded as a potassium glutamate solution. This implies that the concentrations of both ions need to be balanced. While the control of potassium uptake by glutamate is well established for eukaryotic cells, much less is known about the mechanisms that link potassium homeostasis to glutamate availability in bacteria. Here, we have discovered that the availability of glutamate strongly decreases the minimal external potassium concentration required for the highly abundant Bacillus subtilis potassium channel KtrCD to accumulate potassium. In contrast, the inducible KtrAB and KimA potassium uptake systems have high apparent affinities for potassium even in the absence of glutamate. Experiments with mutant strains revealed that the KtrD subunit responds to the presence of glutamate. For full activity, KtrD synergistically requires the presence of the regulatory subunit KtrC and of glutamate. The analysis of suppressor mutants of a strain that has KtrCD as the only potassium uptake system and that experiences severe potassium starvation identified a mutation in the ion selectivity filter of KtrD (Gly282 to Val) that similarly results in a strongly glutamate-independent increase of the apparent affinity for potassium. Thus, this work has identified two conditions that increase the apparent affinity of KtrCD for potassium, i. e. external glutamate or the acquisition of a single point mutation in KtrD.

\section{IMPORTANCE}

In each living cell, potassium is required for maintaining the intracellular $\mathrm{pH}$ and for the activity of essential enzymes. As most other bacteria, Bacillus subtilis possesses multiple low and high affinity potassium uptake systems. Their activity is regulated by the second messenger cyclic di-AMP. Moreover, the pools of the most abundant ions potassium and glutamate must be balanced. We report two conditions, at which the low affinity potassium channel KtrCD is able to mediate potassium uptake at low external potassium concentration: physiologically, the presence of glutamate results in a severely increased potassium uptake. Moreover, this is achieved by a mutation affecting the selectivity filter of the KtrD channel. These results highlight the integration between potassium and glutamate homeostasis in bacteria.

\section{INTRODUCTION}

The cytoplasm of each living cell can be regarded as a potassium glutamate solution. With a concentration between 200 and $400 \mathrm{mM}$, potassium is the most abundant cation, whereas glutamate (100 mM) is the by far most abundant organic molecule (Epstein, 2003; Bennett et al., 2009; Reuß et 
al., 2017). The cells need potassium for the activity of several crucial enzymes and protein complexes, including the ribosome, and for the adjustment of the intracellular $\mathrm{pH}$ by buffering the negative charge of nucleic acids (Epstein, 2003; Rozov et al., 2019). Glutamate is used as building block for proteins and as the central amino group donor for most nitrogen-containing molecules in the cell. Since the affinity of transaminases for glutamate is rather low, a high intracellular concentration is required (Bennett et al., 2009). In addition, both potassium and glutamate play a key role in osmoadaptation in many bacteria: upon exposure to osmotic stress, bacteria very rapidly accumulate potassium as a first response (Bremer and Krämer, 2019). Moreover, glutamate serves as an osmoprotectant in some archaea and bacteria and it is the direct precursor for the rapid biosynthesis of proline if the bacteria experience an osmotic upshift (Bremer and Krämer, 2019). Enteric bacteria accumulate both potassium and glutamate to cope with osmotic stress (Booth and Higgins, 1990).

In the Gram-positive model bacterium Bacillus subtilis, the levels of both molecules are subject to tight regulation. Potassium homeostasis is controlled at the levels of transporter expression and activity. Both mechanisms involve the essential second messenger cyclic di-AMP (c-di-AMP) (Gundlach et al., 2019). Glutamate levels are controlled by a tight regulation of the synthesis of the biosynthetic and degrading enzymes (Commichau et al., 2008). Moreover, the synthesis of glutamine, which is the primary product of ammonium assimilation in B. subtilis, is subject to feedback inhibition (Schumacher et al., 2015). Interestingly, both potassium and glutamate have been implicated in the control of the intracellular levels of c-di-AMP, the major player in the control of potassium homeostasis (Gundlach et al., 2015b; Gundlach et al., 2017b). Moreover, both ions play an important role in osmoprotection, one of the major functions of c-di-AMP (Commichau et al., 2018a; Commichau et al., 2018b; Bremer and Krämer, 2019). Therefore, a regulatory link between the intracellular concentrations of potassium and glutamate has been proposed (Gundlach et al., 2018). However, such a link has so far not been supported by experimental evidence.

B. subtilis encodes three potassium uptake systems, the high- and low-affinity potassium channels KtrAB and KtrCD (Holtmann et al., 2003), respectively, and the high-affinity potassium transporter KimA (Gundlach et al., 2017b). KtrAB and KtrCD consist of a dimer of the transmembrane subunits, KtrB and KtrD, and a cytosolic octameric ring of KtrA and KtrC subunits, respectively (VieiraPires et al., 2013). The cytosolic gating subunits consist of so-called RCK (regulator of conductance of $\mathrm{K}^{+}$) domains that regulate the ion influx (Albright et al., 2006; Szollosi et al., 2016; Diskowski et al., 2017; Schrecker et al., 2019). The expression of the $k \operatorname{trC}$ and $k \operatorname{trD}$ genes encoding the low-affinity potassium channel $K \operatorname{trCD}$ is constitutive (Zhu and Stülke, 2018), whereas the expression of the kimA and $k \operatorname{tr} A B$ genes is barely detectable under standard experimental conditions and is strongly induced upon potassium limitation (Gundlach et al., 2017b; Gundlach et al., 2017a). Both genes are controlled 
by a c-di-AMP-responsive riboswitch that allows transcription beyond the riboswitch only in the absence of c-di-AMP, i. e. under conditions of potassium limitation (Nelson et al., 2013; Gundlach et al., 2017b). However, c-di-AMP does not only interfere with the expression of the high-affinity potassium uptake systems, it does also directly inhibit the activity of all three potassium uptake systems (Rocha et al., 2019; Gundlach et al., 2019). Thus, at high potassium concentration, c-di-AMP accumulates and inhibits the expression and activity of potassium uptake systems. Interestingly, the potassium exporters also possess RCK domains, and these proteins bind c-di-AMP as well. Based on studies with the Staphylococcus aureus potassium exporter $\mathrm{CpaA}$, is has been suggested that c-di-AMP stimulates the activity of the potassium exporters (Chin et al., 2015; Gundlach et al., 2019).

For many Gram-positive bacteria, c-di-AMP is essential for growth on complex medium. However, strains lacking c-di-AMP have been reported for several bacteria including Listeria monocytogenes, S. aureus, Streptococcus agalactiae, and B. subtilis. These mutants grow on specific minimal media, while the presence of osmoprotective compounds is generally toxic for such strains (Whiteley et al., 2015; Gundlach et al., 2017b; Devaux et al., 2018a; Zeden et al., 2018). For B. subtilis strains lacking c-di-AMP potassium is toxic, but the bacteria can handle this toxicity by the acquisition of mutations that either enhance potassium efflux or that reduce potassium uptake (Gundlach et al., 2017b; Gundlach et al., 2019).

To get a more comprehensive understanding of the suggested physiological link between potassium, glutamate, and c-di-AMP, we have studied growth of a strain lacking c-di-AMP in the presence of glutamate. Our results unravel a novel important link between potassium and glutamate: the apparent affinity of the major potassium uptake system KtrCD depends on the presence of glutamate. It has a low apparent affinity for potassium in the absence of glutamate, but a high apparent affinity in its presence. Thus, glutamate and potassium homeostasis are directly coupled to each other in B. subtilis.

\section{RESULTS}

Isolation of suppressor mutants that allow growth of a strain lacking c-di-AMP in the presence of glutamate

A B. subtilis strain lacking all three diadenylate cyclases is unable to grow at potassium concentrations of $5 \mathrm{mM}$ or higher (Gundlach et al., 2017b). It was hypothesized that potassium ions accumulated to toxic concentrations in the mutant. Interestingly, we observed that the c-di-AMP-free strain was also not viable in the presence of glutamate, irrespective of the potassium concentration. We have previously isolated suppressor mutants that can grow without c-di-AMP in the presence of increased potassium concentrations (Gundlach et al., 2017b; Gundlach et al., 2019). One of these suppressor 
mutants was GP2223 that carries a mutation in nhaK, encoding a cation/proton antiporter (Fujisawa et al., 2005). We postulated that the protein variant more actively exports potassium ions thus lowering their internal concentration. Interestingly, also GP2223 was not viable in the presence of glutamate either. To identify the growth-limiting problem, we sought to isolate suppressor mutants that tolerate the presence of glutamate. For this purpose, we plated the c-di-AMP-free strain GP2222 (Gundlach et al., 2017b) on MSSM medium containing glutamate (1\% w/v) and a low concentration of potassium $(0.1 \mathrm{mM} \mathrm{KCl})$. After four days at $37^{\circ} \mathrm{C}$, two colonies could be isolated. One of the strains (GP2840) was subjected to whole genome sequencing to identify the responsible mutations. The suppressor mutant carried an insertion of five base pairs at position 393 of the $k t r C$ gene resulting in a frame shift and the production of a truncated version of the gating subunit KtrC of the potassium channel KtrCD. Moreover, the strain carried point mutations affecting the minor NADH dehydrogenase NdhF, the motility sigma factor SigD, and the phospholipid biosynthetic enzyme PIsC. We tested the second suppressor GP3414 for the presence of these mutations, and found the same mutation in the $k \operatorname{trC}$ gene.

The repeated isolation of mutations affecting the low-affinity potassium uptake system KtrCD was unexpected as this channel is thought to not participate in potassium uptake at the low potassium concentration used for the isolation of the suppressor mutants. However, the obvious selection for the inactivation of KtrCD in the presence of glutamate supports the idea of a relevant physiological link between glutamate and potassium homeostasis. Moreover, the acquisition of mutations affecting the low-affinity potassium channel suggests that KtrCD might be stimulated under the conditions used for the selection, i. e. in the presence of glutamate.

\section{The presence of glutamate differentially affects the activity of $B$. subtilis potassium uptake systems}

B. subtilis is able to grow at potassium concentrations as low as $0.1 \mathrm{mM}$ (Gundlach et al., 2017a). Based on the isolation of $k \operatorname{trC}$ suppressor mutants in the presence of glutamate (see above) we were interested in testing the putative role of glutamate in potassium uptake. To study the contribution of the different potassium uptake systems to growth in the absence or presence of glutamate, we analyzed the growth of $B$. subtilis strains expressing individual potassium uptake systems (Fig. 4.1).

In the absence of glutamate, the wild type strain B. subtilis 168 was viable even at the lowest tested potassium concentration (0.05 mM). In contrast, strain GP1088 lacking all known potassium uptake systems grew only at the highest potassium concentration (50 mM). This observation supports the idea that the three characterized uptake systems are essential to accumulate potassium under physiological conditions. Moreover, $B$. subtilis possesses at least one additional uptake system with a very low apparent affinity for potassium. 
The strains expressing only one of the high-affinity potassium uptake systems KimA or KtrAB were indistinguishable from the wild type strain, i. e. they were able to grow even at very low potassium concentrations. Thus, both KimA and KtrAB alone are sufficient to allow growth of $B$. subtilis in the range of tested potassium concentrations. In contrast, strain GP2165 expressing KtrCD as the single potassium uptake system required at least $0.5 \mathrm{mM}$ of potassium for efficient growth. These observations are in excellent agreement with the previous characterization of KtrAB and KimA as high affinity and of KtrCD as a low affinity potassium uptake system (Gundlach et al., 2017b).

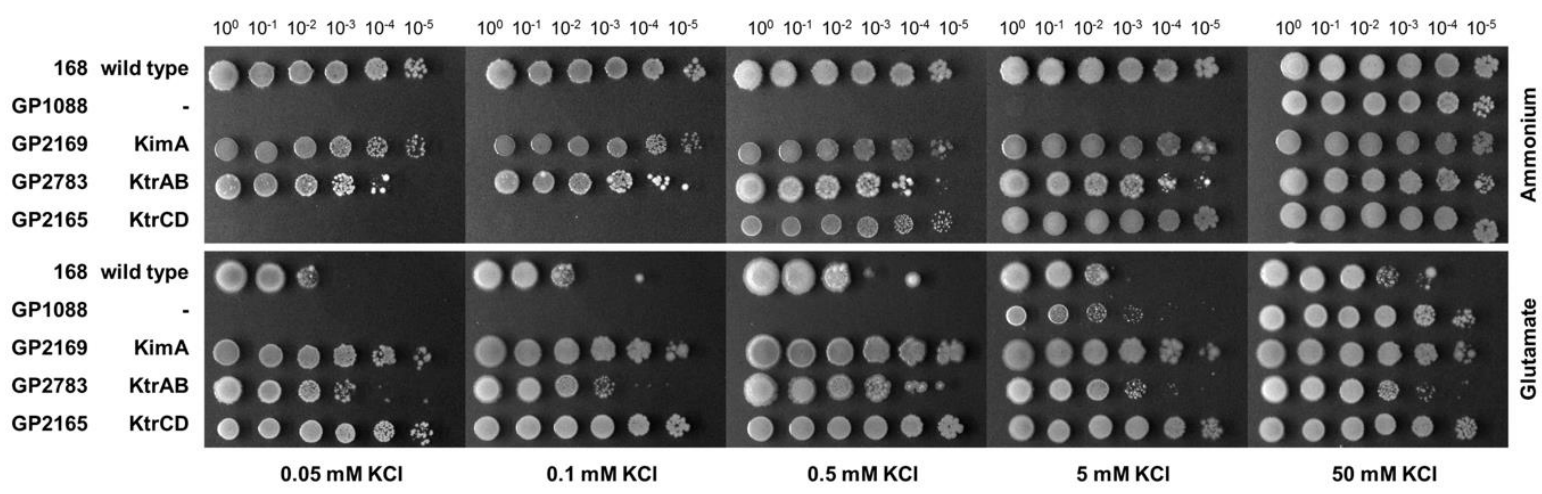

Fig. 4.1. Growth of $B$. subtilis potassium transporter mutants at different potassium concentrations in the presence of ammonium or glutamate. B. subtilis strains expressing one of the potassium uptake systems KimA, $\mathrm{K} \operatorname{tr} \mathrm{AB}$, or $\mathrm{KtrCD}$, or none at all were cultivated in MSSM minimal medium with $50 \mathrm{mM} \mathrm{KCl}$ and ammonium. Cells were harvested, washed, and the $\mathrm{OD}_{600}$ was adjusted to 1.0. Serial dilutions were dropped onto MSSM minimal plates with the indicated potassium concentration and ammonium or glutamate.

Growth of the strains on minimal media containing glutamate gave different results: Again, the wild type strain as well as the mutants carrying one of the high affinity uptake systems, KimA or KtrAB, were able to grow even at the lowest potassium concentration. However, in contrast to the results obtained for growth in the absence of glutamate, the strain expressing KtrCD as the only potassium uptake system did now also grow at the lowest tested potassium concentration. These observations were also confirmed by testing the growth of the strains expressing one single potassium uptake system each in liquid medium (see Supplementary Figure S4.1). In the experiments described above, glutamate was added as the sodium salt. To exclude the possibility that sodium was the effector of KtrCD, we cultivated B. subtilis GP2165 that has KtrCD as the only potassium uptake system under conditions of potassium limitation (no glutamate, $0.5 \mathrm{mM} \mathrm{KCl}$ or no $\mathrm{KCl}$ ) and added potassium chloride, potassium glutamate, sodium chloride, or sodium glutamate to the culture after the cessation of growth (see Fig. 4.2). The bacteria immediately resumed growth upon the addition of potassium chloride or potassium glutamate indicating that the lack of potassium was the growth-limiting factor. 
After a longer lag phase the addition of sodium glutamate also allowed continued growth, but only in the presence of $0.5 \mathrm{mM} \mathrm{KCl}$ in the medium. In contrast, no restoration of growth was possible for the culture without added $\mathrm{KCl}$. Taken together, all these data clearly demonstrate that glutamate stimulates the ability of KtrCD to take up potassium at very low concentrations of the ion.
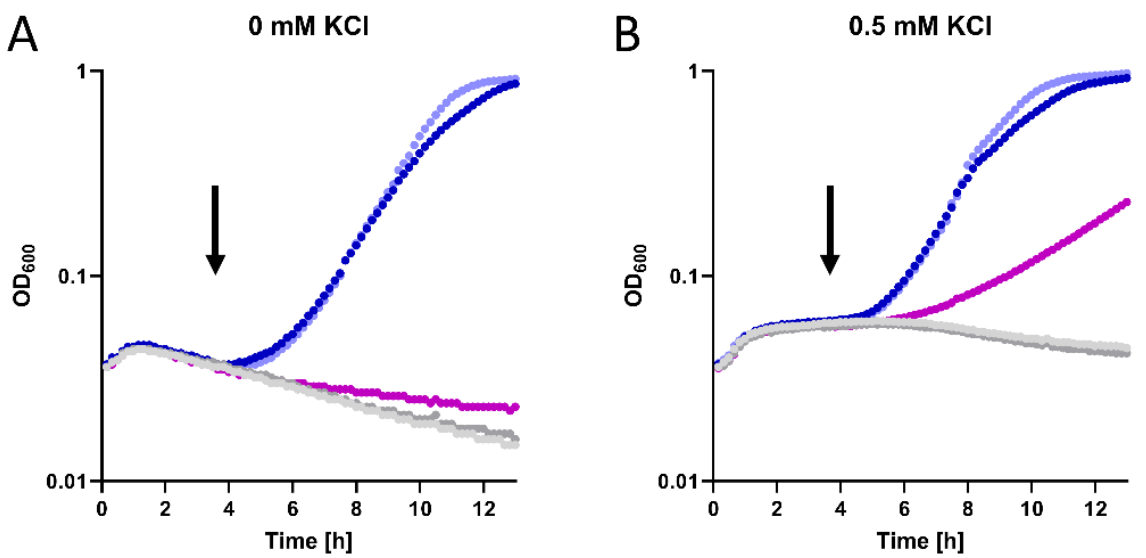

buffer

- $\mathrm{NaCl}[5 \mathrm{mM}]$

- $\mathrm{KCl}[5 \mathrm{mM}]$

- Na-glutamate [5 mM]

- K-glutamate [5mM]

Fig. 4.2. Activity of KtrCD upon potassium limitation. B. subtilis GP2165 expressing only KtrCD was grown in MSSM medium with ammonium and $0.5 \mathrm{mM} \mathrm{KCl}(A)$ or $0 \mathrm{mM} \mathrm{KCl}(B)$ and growth was monitored. As soon as the cells reached stationary growth phase as a result of potassium starvation, different substances were added and growth was monitored further. Added compounds were: MSSM base buffer, $\mathrm{NaCl} 5 \mathrm{mM}, \mathrm{KCl} 5 \mathrm{mM}$, sodium glutamate $5 \mathrm{mM}$, and potassium glutamate.

In order to study the effect of glutamate on KtrCD in more detail, we analyzed the activity of the protein in a heterologous complementation assay using E. coli LB2003 (Stumpe and Bakker, 1997). This strain is deficient in the three major endogenous potassium uptake systems Trk, Kup, and Kdp, and is therefore not able to grow at low potassium concentrations without complementation using a gene encoding a potassium uptake system. For the IPTG-inducible expression of $k \operatorname{tr} C D$ we used plasmid pGP2989. Accordingly, E. coli LB2003 was transformed with this plasmid or the empty vector control pWH844 (Schirmer et al., 1997) and growth was assessed in minimal medium supplemented with increasing $\mathrm{KCl}$ concentrations $(0.001,0.01,0.02,0.04,0.06,0.1,0.2,0.5,10$ and $50 \mathrm{mM} \mathrm{KCl}$ ) (see Fig. 4.3). While $50 \mathrm{mM} \mathrm{KCl}$ were required for growth of the strain carrying the empty vector (data not shown), expression of KtrCD allowed growth at much lower $\mathrm{KCl}$ concentrations. The determination of the growth rates at different potassium concentrations allowed fitting according to the Monod equation, an equation describing the growth of cultures, which is based on the Michaelis-Menten equation (Monod, 1949). This revealed the maximum specific growth rate $\mu_{\max }\left[\mathrm{h}^{-1}\right]$ and the substrate concentration that supports the half-maximal growth rate $\mathrm{K}_{\mathrm{s}}[\mathrm{mM} \mathrm{KCl}]$ of $\mathrm{KtrCD}$ in the absence or presence of glutamate. The mean $\mathrm{K}_{\mathrm{s}}$ values of three independent biological replicates are as follows: 
In the absence of glutamate, $K \operatorname{trCD}$ had a $\mathrm{K}_{\mathrm{s}}$ of $0.278 \pm 0.023 \mathrm{mM}$ for potassium, and a $\mu_{\max }$ of $0.71 \pm 0.043 \mathrm{~h}^{-1}$. In contrast, the presence of glutamate in the medium resulted in a $\mathrm{K}_{\mathrm{s}}$ for potassium of $0.017 \pm 0.0045 \mathrm{mM}$ and a $\mu_{\max }$ of $0.374 \pm 0.051 \mathrm{~h}^{-1}$. This 16 -fold increase of the apparent affinity for potassium in the presence of glutamate perfectly fits with the growth phenotype observed in $B$. subtilis mutants and might suggest that the presence of glutamate converts KtrCD from a low- to a high-affinity potassium uptake system.

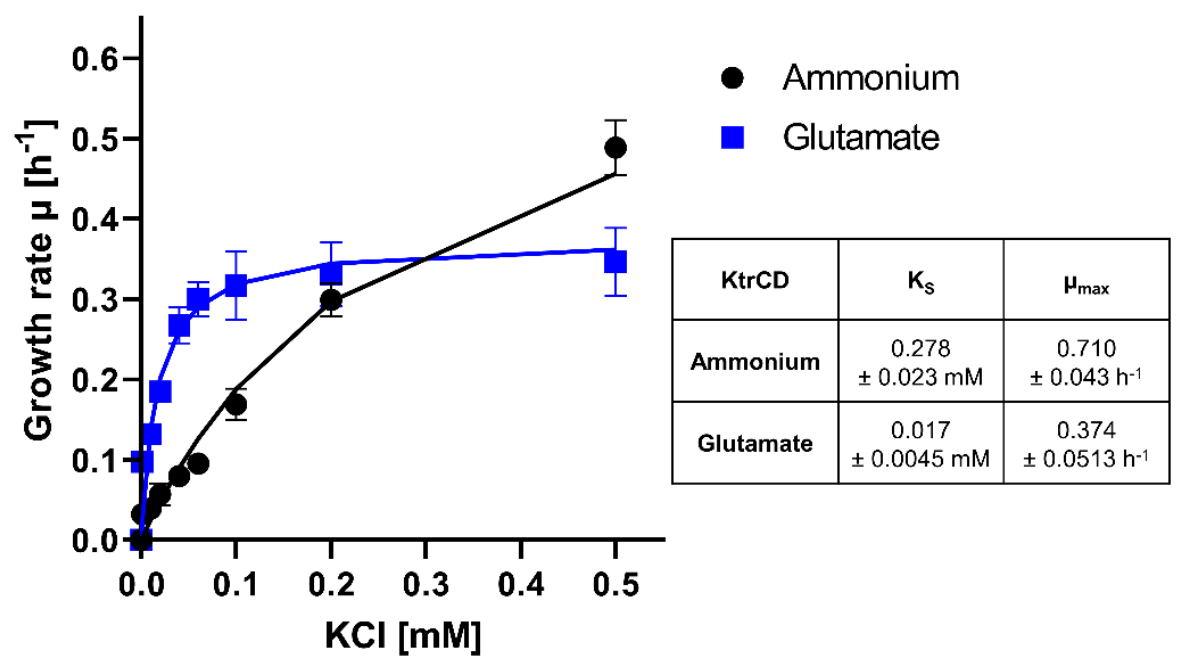

Fig. 4.3. Activity of KtrCD in a heterologous complementation assay using $E$. coli 2003 reveals the impact of glutamate. LB2003 was transformed with pGP2989, and growth at different potassium concentrations with ammonium or glutamate was assessed over $24 \mathrm{~h}$. The growth rate was determined and plotted against the potassium concentrations. The lines show the ideal progression of $\mu$ over the different potassium concentrations according to the Monod equation. Standard deviations derived from three independent experiments are indicated.

\section{Contribution of the membrane and gating subunits to the regulation by glutamate}

The Ktr potassium channels consist of an integral membrane and a gating subunit. In order to study the role of the subunits in the control of KtrCD by glutamate, we constructed two series of strains: $A$ first set encoded only one of the transmembrane subunits $\operatorname{KtrB}$ or $\operatorname{KtrD}$, whereas the second set expressed Ktr channels with swapped subunits (i. e. KtrCB and KtrAD).

The strains expressing a single transmembrane subunit ( $\operatorname{trB}$ or $K \operatorname{trD})$ were unable to grow at low potassium concentrations (below $0.5 \mathrm{mM}$ ) in medium with ammonium as the nitrogen source (see Fig. 4.4). However, they exhibited good growth at $5 \mathrm{mM}$ potassium. If glutamate was present in the medium, both KtrB and KtrD allowed growth even at the lowest potassium concentrations. These observations demonstrate that KtrB and KtrD are functional potassium channels even in the absence 
of a gating subunit. Moreover, both $\mathrm{KtrB}$ and KtrD appear to become more affine for potassium in the presence of glutamate.

It has been shown before that Ktr channels with swapped subunits are active in potassium uptake (Rocha et al., 2019). Here, we tested the response of such channels to the availability of glutamate (Fig. 4.4). Strain GP3082 encoding KtrCB was indistinguishable from strain GP2783 (KtrAB) both in the absence and presence of glutamate. The growth of these strains even at the lowest tested potassium concentrations confirmed that KtrB is the major determinant for the high affinity to potassium. The growth pattern of the strain expressing KtrAD (GP3127) was similar to the isogenic strain encoding KtrCD (GP2165). In the absence of glutamate, KtrAD allowed growth only at elevated potassium concentrations. This was even more pronounced as compared to the cognate KtrCD

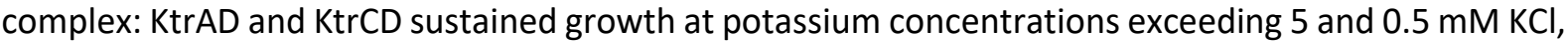
respectively. In the presence of glutamate, both strains grew well at all tested potassium concentrations. These observations indicate that KtrD is the determinant for the apparently low affinity to potassium in the absence of glutamate. Moreover, the cognate complex between KtrD and $\mathrm{KtrC}$ is more efficient in potassium uptake in the absence of glutamate as compared to the KtrD-KtrA complex.

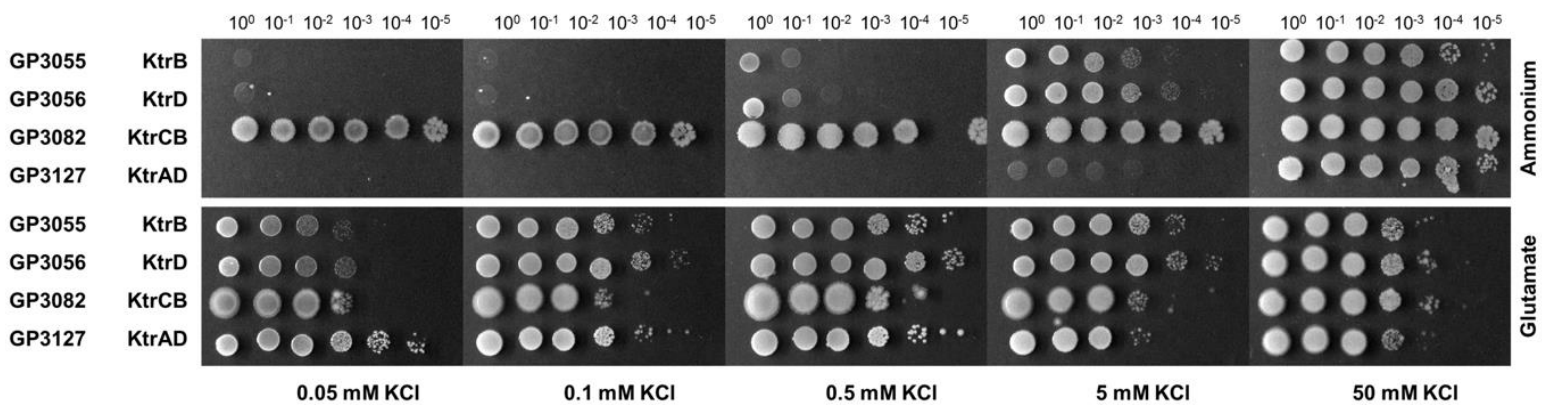

Fig. 4.4. Growth of $B$. subtilis potassium transporter mutants at different potassium concentrations in the presence of ammonium or glutamate. $B$. subtilis mutants expressing one of the transmembrane subunits KtrB and KtrD or the hybrid channels KtrCB or KtrAD as the single potassium uptake system were cultivated in MSSM minimal medium with $50 \mathrm{mM} \mathrm{KCl}$ and ammonium. Cells were harvested, washed, and the $\mathrm{OD}_{600}$ was adjusted to 1.0. Serial dilutions were dropped onto MSSM minimal plates with the indicated potassium concentration and ammonium or glutamate.

Taken together, the experiments with both the isolated membrane subunits as well as with the cognate and swapped Ktr complexes demonstrate that both membrane subunits have a higher apparent affinity for potassium in the presence of glutamate. The presence of either of the two gating subunits strongly enhanced the apparent affinity of KtrB to potassium even in the absence of glutamate thus resulting in highly affine KtrAB and KtrCB complexes, respectively. Thus, in the case of 
KtrB, the gating subunits enhance the affinity of the channel to potassium irrespective of the presence of glutamate. In contrast, for KtrD, the association with either gating subunit resulted in enhanced activity, but at low potassium concentrations, KtrD remained fully dependent on the presence of glutamate even if bound to one of the gating subunits.

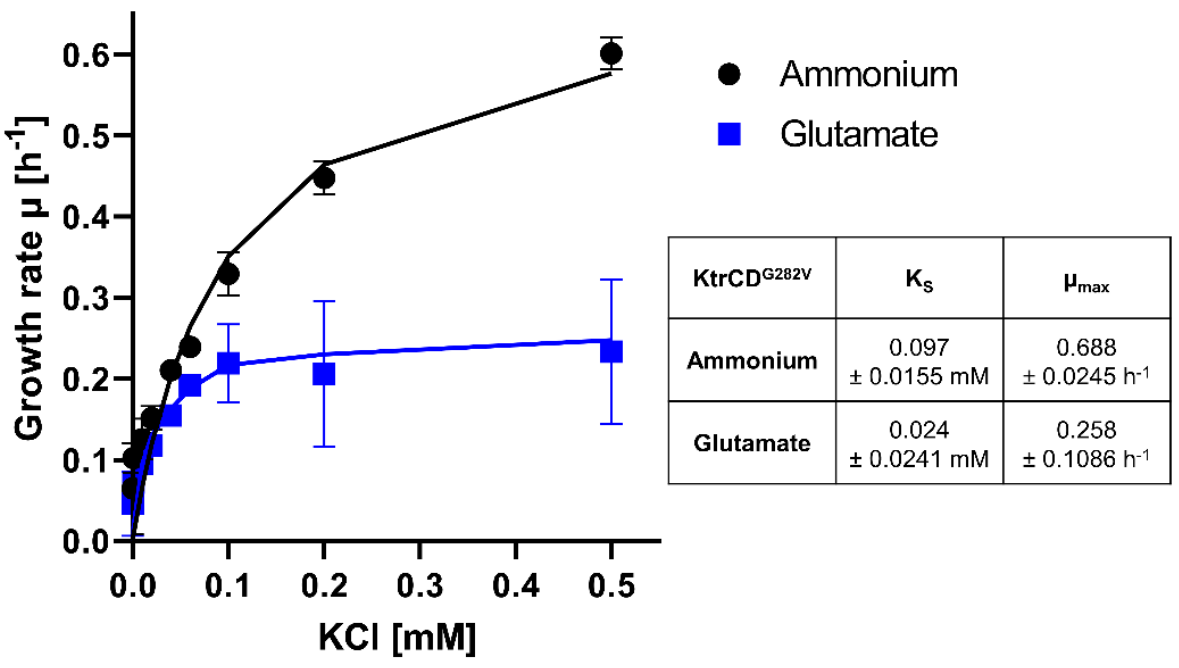

Fig. 4.5. A single amino acid exchange, G282V in KtrD, confers high affinity for potassium independent of glutamate to KtrCD. E. coli LB2003 was transformed with pGP3303, and growth at different potassium concentrations with ammonium or glutmate was assessed over $24 \mathrm{~h}$. The growth rate was determined and plotted against the potassium concentrations. The lines show the ideal progression of $\mu$ over the different potassium concentrations according to the Monod equation. Standard deviations derived from three independent experiments are indicated.

\section{Identification and characterization of a high-affinity variant of KtrD}

The results presented above suggest that specific features of KtrD cause the low affinity for potassium in the absence of glutamate even if the protein forms a complex with its cognate gating subunit KtrC. We have observed that strains lacking the high affinity uptake systems KimA and KtrAB experience a severe potassium limitation when cultivated at low concentrations of $\mathrm{KCl}$. To cope with this limitation, such strains typically acquire mutations that result in the overexpression of the arginine biosynthetic pathway to substitute for the positive charge normally provided by potassium ions (Gundlach et al., 2017a). However, such suppressors are unable to grow in the absence of the c-di-AMP receptor protein DarA (Gundlach et al., 2015a; Weiß, 2018). Hence, a strain lacking KimA, KtrAB and DarA (GP2495) was used to select suppressor mutants that are able to grow at low potassium concentrations $(0.5 \mathrm{mM}$ $\mathrm{KCl}$ ). We expected that such mutants would allow either the uptake of potassium at low concentrations or activate alternative pathways to provide positively charged molecules. Whole genome sequencing of one of the mutants (GP3100) revealed a point mutation in the ktrD gene, resulting in an exchange 
of a glycine at position 282 by a valine. This residue is part of the selectivity filter and at the same time located close to the intramembrane loop of KtrD, which serves as gate (Diskowski et al., 2015). Interestingly, the selectivity filters of related proteins (including KtrB) have a highly conserved R-T/SA-G motif at this position, whereas KtrD has an R-S-G-G motif. The replacement of the first glycine by a larger amino acid (valine) makes the selectivity filter thus more similar to those in the other potassium channels at this position.

To determine the activity of the mutant $\mathrm{KtrCD}^{\mathrm{G} 282 \mathrm{~V}}$ channel more precisely, we performed the heterologous complementation assay using E. coli LB2003 as described above (Fig. 4.5). In the presence of ammonium, $\operatorname{KtrCD}^{\mathrm{G} 282 \mathrm{~V}}$ had a $\mathrm{K}_{\mathrm{s}}$ of $0.097 \pm 0.015 \mathrm{mM}$ for potassium, and a $\mu_{\max }$ of $0.69 \pm 0.024 \mathrm{~h}^{-1}$. The presence of glutamate in the medium resulted in an apparent $\mathrm{K}_{\mathrm{s}}$ for potassium of $0.024 \pm 0.024 \mathrm{mM}$ and a $\mu_{\max }$ of $0.258 \pm 0.109 \mathrm{~h}^{-1}$. This result confirms the increased apparent affinity of the mutant channel for potassium as compared to the wild type protein.

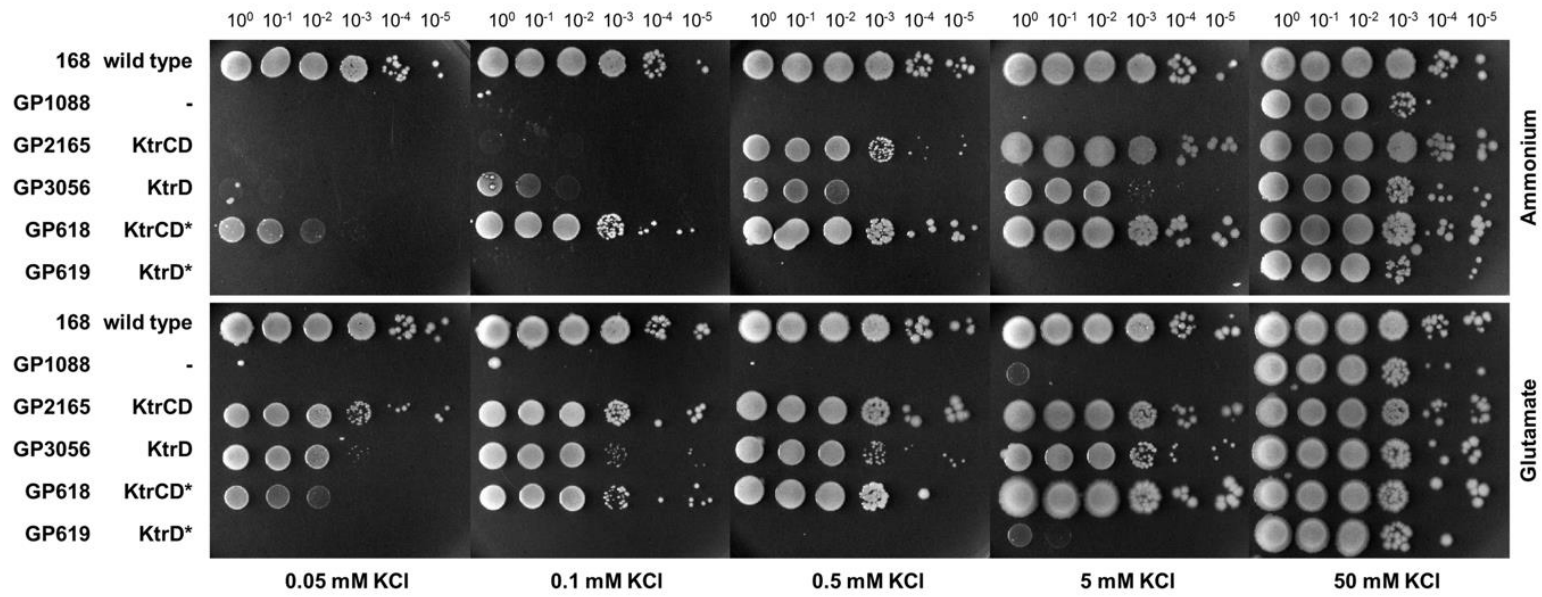

Fig. 4.6. Growth experiments with KtrCD variants highlight the relevance of the G282V mutation for the physiology of $B$. subtilis. B. subtilis strains expressing $K \operatorname{trCD}, K \operatorname{trD}$ and the mutated variants as the only potassium uptake systems were cultivated in MSSM minimal medium with $50 \mathrm{mM} \mathrm{KCl}$ and ammonium. Cells were harvested, washed, and the $\mathrm{OD}_{600}$ was adjusted to 1.0. Serial dilutions were dropped onto MSSM minimal plates with the indicated potassium concentration and ammonium or glutamate. The mutation in the $k \operatorname{tr} D$ gene was introduced into the chromosome via CRISPR-Cas9 gene editing.

The mutant $\mathrm{KtrD}^{\mathrm{G282V}}$ protein may have a high apparent affinity for potassium in the presence of the cognate gating subunit irrespective of the presence of glutamate, as observed for KtrB. Alternatively, and unlike KtrB, the mutant protein may have an intrinsic high apparent affinity for potassium, independent of the cognate gating subunit. To distinguish between these two possibilities, we used CRISPR/Cas9-mediated genome engineering to construct the strains GP618 and GP619, which had $\operatorname{KtrCD}^{\mathrm{G} 282 \mathrm{~V}}$ and $\mathrm{KtrD}{ }^{\mathrm{G} 282 \mathrm{~V}}$, respectively, as the only potassium uptake systems. The activity of these 
channels was studied by assaying growth of the $B$. subtilis strains at different potassium concentrations in the presence or absence of glutamate (Fig. 4.6). As expected, the strain expressing $\operatorname{KtrCD}^{6282 \mathrm{~V}}$ grew even at the lowest tested potassium concentration both in the presence or absence of glutamate. In contrast, strain GP619 with $\mathrm{KtrD}^{\mathrm{G282V}}$ as the single potassium channel was unable to grow at low potassium concentrations. Even in the presence of glutamate, the wild type KtrD was active at low potassium concentrations, we observed no growth at a potassium concentration lower than $50 \mathrm{mM}$ $\mathrm{KCl}$. As for the strain GP1088 lacking all known potassium uptake systems, GP619 was viable in the presence of $50 \mathrm{mM} \mathrm{KCl}$ whether glutamate was present or not. This finding suggests that the mutant channel protein is inactive in the absence of its gating subunit $\mathrm{KtrC}$, while in its presence $\mathrm{Ktr} C D^{\mathrm{G} 282 \mathrm{~V}}$ shows a high glutamate-independent affinity for potassium. Thus, the mutant $K \operatorname{tr} C D^{6282 \mathrm{~V}}$ resembled the KtrAB complex in the requirement for the gating subunit for glutamate-independent potassium uptake at low concentrations of the ion.

\section{DISCUSSION}

Potassium and glutamate are essential for any living cell, and their concentrations have to be balanced (Gundlach et al., 2018). Indeed, glutamate-sensing potassium transporters are widespread in eukaryotic cells (Sobolevsky, 2015) but are present only in few prokaryotes, such as the cyanobacteria (Ger et al., 2010). Here, we demonstrate that control of potassium uptake by glutamate can also be achieved by a classical bacterial potassium channel, $\operatorname{KtrCD}$, which depends on glutamate for the efficient potassium uptake at low external concentrations of the cation. This link between glutamate and $\operatorname{KtrCD}$ was first observed when we cultivated a B. subtilis strain lacking c-di-AMP in the presence of glutamate. To our surprise, this strain carried a mutation that resulted in inactivation of $\mathrm{KtrC}$. With the results presented in this study, we can suggest that glutamate was toxic for the strain lacking c-diAMP since it stimulated potassium accumulation to a toxic level. This is in excellent agreement with the idea that the control and limitation of potassium uptake is an essential function of c-di-AMP (Gundlach et al., 2017b; Gundlach et al., 2019).

Recent structural analyses of the gating subunit KtrC in the presence of ATP and ADP uncovered a ligand-dependent assembly/ disassembly of the gating ring. These observations already suggested a distinct physiological role of the KtrCD system when compared to KtrAB and KimA (Diskowski et al., 2017). This role may lay in the link between the glutamate and the potassium homeostasis. In contrast to KtrAB and KimA, KtrCD is strongly constitutively expressed in B. subtilis (Zhu and Stülke, 2018) and can readily be activated. In the absence of glutamate, KtrCD serves the general potassium homeostasis. Additionally, we here established that in the presence of a high external glutamate concentration, the apparent affinity of KtrCD for potassium increases 16 -fold, 
allowing the accumulation of potassium even if limited on the outside. Prior to this stimulation, glutamate likely is taken up into the cells as deduced from the delayed growth initiation after potassium starvation (see Fig. 4.2a). The resulting imbalance between negative and positive charges may consequently require the rapid uptake of potassium ions to counterbalance the negative charges. This immediate uptake could always be achieved by the constitutively present $K \operatorname{trCD}$. Binding of glutamate or a downstream metabolite of it may than result in the more effective uptake of potassium ions. Alternatively, an increased membrane potential resulting from the increased utilization of glutamate could promote potassium fluxes through $K \operatorname{trCD}$. Due to the more efficient potassium uptake in the presence of glutamate, it also becomes obvious why KtrCD is inactivated by the direct binding of c-di-AMP: too high internal potassium concentrations accumulated via KtrCD would again be toxic for the cells (Rocha et al., 2019; Gundlach et al., 2019).

Interesting future questions are of course how glutamate stimulates the potassium uptake through KtrCD and what distinguishes the KtrCD system from the paralogous KtrAB potassium channel. KtrD and KtrB share $37 \%$ identity and are predicted to be structurally very similar. KtrC and KtrA are $55 \%$ identical and both form octameric rings that associate to the dimeric channel subunits. Despite the apparently high similarity of both systems, $\mathrm{KtrCD}$ was assumed to be tenfold less affine for $\mathrm{K}^{+}$ (Holtmann et al., 2003). This lower apparent affinity could result either from a lower binding affinity at the slightly different selectivity filter or from a second selective step, the gating. In KtrAB, KtrA facilitates the gating of the channel, while KtrB initially binds $\mathrm{K}^{+}$at its selectivity filter (Tholema et al., 2005; Kröning et al., 2007; Vieira-Pires et al., 2013; Diskowski et al., 2017). It has recently been shown that it is in fact the gating that determines the high apparent affinity of KtrAB: while potassium ions also bind to KtrB alone with micromolar affinity (low $K_{D}$ value), a low $K_{M}$ value in the micromolar range was only observed in the presence of the gating subunit KtrA and sodium ions (Mikušević et al., 2019). The respective $\mathrm{K}_{\mathrm{M}}$ values for $\mathrm{KtrB}$ and $\mathrm{KtrAB}$ in the presence of $\mathrm{Na}^{+}$are in good agreement to the apparent affinities deduced from growth curves ( $K_{s}$ values) shown here (see Fig. 4.1). In analogy to the stimulatory effect of $\mathrm{Na}^{+}$on $\mathrm{Ktr} A B$, it is tempting to speculate that internal glutamate might play a similar role for $K \operatorname{trCD}$. By the binding to either the regulatory subunit $\mathrm{KtrC}$ or the gating region of KtrD, the gating could be facilitated allowing for the efficient potassium uptake even at very low external concentrations. Our data favor the binding to the gating region since an increased apparent affinity was observed in the presence of glutamate already for KtrD alone. Yet, the isolation of KtrC suppressor mutants of a c-di-AMP free strain in the presence of glutamate suggests a significant contribution of $\mathrm{KtrC}$ to the glutamate-dependent gating and its role is further reinforced by the improved growth in the presence of $\mathrm{KtrC}$ and glutamate at low external potassium concentrations. Gating may thus underlay a synergistic effect between the glutamate binding to KtrD and the regulatory effect of KtrC. 
In agreement with this hypothesis are the results of the selectivity filter mutant in KtrD: the selectivity filter and the gate are in close proximity and the identified mutation (G282V) is located on the inner side of the filter. The Gly-to-Val exchange may thus facilitate the KtrC-mediated gating, rendering KtrCD independent of glutamate, while in the absence of $K \operatorname{tr} C$ the gate opens less efficient than in wildtype KtrD.

The idea of a link between glutamate and potassium homeostasis is not entirely new for bacteria (Chen et al., 1999; Ger et al., 2010). Glutamate receptors sense an increased external glutamate concentration leading to transporter activation. However, the physiological role of bacterial glutamate receptors has not been established. A potassium channel suggested to sense the internal glutamate concentration is the B. subtilis potassium exporter YugO (Prindle et al., 2015). YugO is proposed to function in electrical signaling within a bacterial biofilm. Upon glutamate starvation in the interior cells of a biofilm, the channel would be activated resulting in the efflux of potassium ions. This efflux consequently would depolarize the neighboring outer cells, which thus would consume less glutamate. Without any loss of signal intensity the electrical signal would propagate through a biofilm making glutamate more accessible for the interior cell. For the benefit of the exterior cells the interior cells in return would provide sufficient concentrations of ammonium allowing the survival of the whole biofilm (Prindle et al., 2015; Huang, 2017). KtrCD appears to function in the opposite direction by accumulating potassium ions, if high internal concentrations of glutamate are present. KtrCD could consequently be involved in the signaling within bacterial biofilms. It may serve the second step, the uptake of glutamate by the hyperpolarized interior cells, by allowing for the rapid accumulation of both, potassium and glutamate. This way, the interior cells could accumulate the required glutamate and restore their potassium pool.

It is striking that the B. subtilis potassium import and export systems, KtrCD and YugO, are stimulated and inhibited, respectively, by glutamate. Moreover, the integration of potassium and glutamate homeostasis by the essential second messenger c-di-AMP suggests that we are only starting to discover the tip of an iceberg. A complete picture of the interdependency between glutamate and potassium concentrations and the underlying regulatory mechanisms will be essential for full understanding of living cells.

\section{EXPERIMENTAL PROCEDURES}

\section{Strains, media and growth conditions}

E. coli DH5 $\alpha$ (Sambrook and Russell, 2001) was used for cloning and for the expression of recombinant proteins. E. coli LB2003 (Stumpe and Bakker, 1997) was used to assay potassium uptake activity. All B. subtilis strains used in this study are derivatives of the laboratory strain 168 . They are listed in 
Chapter 9. B. subtilis was grown in Luria-Bertani (LB) or in sporulation (SP) medium (Sambrook and Russell, 2001; Kunst and Rapoport, 1995). For the assay of potassium channel activity, B. subtilis and E. coli were cultivated in MSSM medium (Gundlach et al., 2017b). In this medium $\mathrm{KH}_{2} \mathrm{PO}_{4}$ was replaced by $\mathrm{NaH}_{2} \mathrm{PO}_{4}$ and $\mathrm{KCl}$ was added as indicated. The media were supplemented with ampicillin $(100 \mu \mathrm{g} /$ $\mathrm{ml})$, kanamycin (10 and $50 \mu \mathrm{g} / \mathrm{ml}$ for $B$. subtilis and E. coli, respectively), chloramphenicol $(5 \mu \mathrm{g} / \mathrm{ml})$, tetracyclin $(12.5 \mu \mathrm{g} / \mathrm{ml})$, spectinomycin $(150 \mu \mathrm{g} / \mathrm{ml})$ or erythromycin and lincomycin $(2$ and $25 \mu \mathrm{g} / \mathrm{ml}$, respectively) if required.

\section{DNA manipulation and genome sequencing}

Transformation of E. coli and plasmid DNA extraction were performed using standard procedures (Sambrook and Russell, 2001). All commercially available plasmids, restriction enzymes, T4 DNA ligase and DNA polymerases were used as recommended by the manufacturers. Chromosomal DNA of B. subtilis was isolated as described (Kunst and Rapoport, 1995). B. subtilis was transformed with plasmid and genomic DNA according to the two-step protocol (Kunst and Rapoport, 1995). To identify the mutations in the suppressor mutant strains GP2840 and GP3100, their genomic DNA was subjected to whole-genome sequencing (Reuß et al., 2017). Briefly, the reads were mapped on the reference genome of B. subtilis 168 (GenBank accession number: NC_000964) (Barbe et al., 2009). Mapping of the reads was performed using the Geneious software package (Biomatters Ltd., New Zealand) (Kearse et al., 2012). Single nucleotide polymorphisms were considered as significant when the total coverage depth exceeded 25 reads with a variant frequency of $\geq 90 \%$. All identified mutations were verified by PCR amplification and Sanger sequencing.

\section{Construction of mutant strains by allelic replacement}

Deletion of the $\operatorname{dar} A, \operatorname{kim} A, k \operatorname{tr} A$ and $k \operatorname{tr} B$ genes as well as of the $k \operatorname{tr} A B$ operon was achieved by transformation of $B$. subtilis 168 with a PCR product constructed using oligonucleotides to amplify DNA fragments flanking the target genes and an appropriate intervening resistance cassette as described previously (Diethmaier et al., 2014). The integrity of the regions flanking the integrated resistance cassette was verified by sequencing PCR products of about 1,000 bp amplified from chromosomal DNA of the resulting mutant strains.

\section{Genome editing}

A single amino acid substitution in $\operatorname{KtrD}(\mathrm{G} 282 \mathrm{~V})$ was generated at the native $k \operatorname{trD}$ locus using CRISPR editing as described (Altenbuchner, 2016). Briefly, oligonucleotides encoding a 20 nucleotide gRNA with flanking Bsal sites and a repair fragment carrying mutations of interest with flanking Sfil restriction 
sites were cloned sequentially into vector pJOE8999 (Altenbuchner, 2016) and the resulting plasmid pGP2833 was used to transform recipient $B$. subtilis strain 168 and cells were plated on $15 \mu \mathrm{g} / \mathrm{ml}$ kanamycin plates with $0.2 \%$ mannose. Transformation was carried out at $30^{\circ} \mathrm{C}$ since replication of pJOE8999 derivatives is temperature-sensitive. The transformants were patched on LB agar plates and incubated at the non-permissive temperature of $50^{\circ} \mathrm{C}$. The loss of the vector was verified by the inability of the bacteria to grow on kanamycin plates. The presence of the desired mutations in the resulting strain GP616 was confirmed by Sanger sequencing.

\section{Plasmid constructions}

The $k \operatorname{tr} D$ alleles were amplified using chromosomal DNA of $B$. subtilis 168 or GP3100 as the template and appropriate oligonucleotides that attached Pstl and HindIII sites and a ribosomal binding site to the ktrD fragment. The ktrD alleles were cloned between the Pstl and HindIII sites of pGP2907 (Gundlach et al., 2019) resulting in pGP2989 and pGP3303. These plasmids allow the expression of wild type and mutant $\left(K \operatorname{tr} D^{G 282 V}\right) k \operatorname{trCD}$ operons, respectively, in E. coli.

\section{Determination of specific growth parameters}

The growth characteristics of $E$. coli LB2003 complemented with plasmid-based $K \operatorname{trCD}, \operatorname{KtrCD}^{\mathrm{G} 282 \mathrm{~V}}$ or empty vector were determined as follows. Potassium was used as the growth-limiting factor. The bacteria were inoculated in LB medium containing $50 \mathrm{mM} \mathrm{KCl}$ and precultured in MSSM medium, supplemented with thiamin $(1 \mathrm{mg} / \mathrm{ml})$ and $50 \mathrm{mM} \mathrm{KCl}$. The cultures were grown until exponential phase, harvested, and the cells were incubated for $1 \mathrm{~h}$ in potassium-free medium, and washed three times in MSSM buffer. Afterwards, the cells were adjusted to $\mathrm{OD}_{600} 1.0$ and used to inoculate a 96 well plate (Microtest Plate 96 Well, Sarstedt) containing MSSM medium with either ammonium or glutamate and the required potassium concentrations. Growth was tracked in an Epoch 2 Microplate Spectrophotometer (BioTek Instruments) at $37^{\circ} \mathrm{C}$ with linear shaking at $237 \mathrm{cpm} \mathrm{(4} \mathrm{mm)} \mathrm{for} 20 \mathrm{~h}$, and optical density at $600 \mathrm{~nm}$ was measured in $10 \mathrm{~min}$ intervals. The exponential growth phase was used to determine the growth rate $\mu\left[\mathrm{h}^{-1}\right]$. The growth rates were then plotted against the potassium concentrations. This allowed fitting to the Monod equation and calculation of $\mu_{\max }\left[\mathrm{h}^{-1}\right]$ and the apparent $\mathrm{K}_{\mathrm{s}}[\mathrm{mM} \mathrm{KCl}]$ using the solver tool of Excel 2012 (Microsoft). Experiments were repeated with three biological replicates.

To assay growth of $B$. subtilis mutants at different potassium concentrations, a drop dilution assay was performed. Briefly, precultures in MSSM medium with ammonium and $50 \mathrm{mM} \mathrm{KCl}$ were washed three times and the cells were adjusted to an $\mathrm{OD}_{600}$ of 1.0 in MSSM buffer. Dilution series were 
then spotted onto MSSM plates with ammonium or glutamate and different $\mathrm{KCl}$ concentrations $(0.001$, $0.01,0.02,0.04,0.06,0.1,0.2,0.5,10$ and $50 \mathrm{mM} \mathrm{KCl})$.

For the determination of growth characteristics in liquid medium, cells were treated as described above and the cells were adjusted to an $\mathrm{OD}_{600}$ of 1.0 and used to inoculate a 96 well plate (Microtest Plate 96 Well, Sarstedt) containing MSSM medium with either ammonium or glutamate and the required potassium concentration.

\section{SUPPLEMENTARY MATERIAL}

https://jb.asm.org/content/202/12/e00138-20

Fig. S4.1. Growth of B. subtilis potassium transporter mutants at different potassium concentrations in the presence of ammonium or glutamate.

\section{ACKNOWLEDGMENTS}

We would like to thank Iljana Gerlitzki, Julius Fülleborn, Jan Gundlach, and Alexander Lockhorn for the help with strain construction. This work was supported by a grant from the Deutsche Forschungsgemeinschaft via Priority Program SPP1879 (to I.H. and J.S.).

\section{CONFLICT OF INTEREST}

The authors declare that they have no conflicts of interest with the contents of this article. 


\section{Chapter 5 - Bypassing cyclic di-AMP essentiality}

The results described in this chapter were originally published as a preprint on bioRxiv (doi: https://doi.org/10.1101/2020.09.03.280651):

\section{Essentiality of c-di-AMP in Bacillus subtilis: Bypassing mutations converge in potassium and glutamate homeostasis}

Larissa Krüger ${ }^{1}$, Christina Herzberg ${ }^{1}$, Hermann Rath ${ }^{2}$, Tiago Pedreira ${ }^{1}$, Anja Poehlein ${ }^{3}$, Jan Gundlach ${ }^{1}$, Rolf Daniel ${ }^{3}$, Uwe Völker², Ulrike Mäder², and Jörg Stülke ${ }^{1}$

${ }^{1}$ Department of General Microbiology, GZMB, Georg-August-University Göttingen, Germany

2 Interfaculty Institute for Genetics and Functional Genomics, University Medicine Greifswald, Greifswald, Germany

${ }^{3}$ Department of Genomic and Applied Microbiology, GZMB, Georg-August-University Göttingen, Germany

\section{AUTHOR CONTRIBUTIONS}

LK and JS conceived the study and designed the experiments. LK and CH isolated and characterized the suppressor mutant strains. LK and $\mathrm{CH}$ conducted the kinetic experiments that led to characterization of the novel glutamate transporter AimA. JG and CH prepared cultures for the transcriptomic analysis. $\mathrm{HR}, \mathrm{UV}$, and UM performed the transcriptomic analysis and analyzed the data, and TP represented the data. AP and RD performed whole genome sequencing. LK analyzed the whole genome sequencing data. LK and JS wrote the manuscript. All authors approved the manuscript. 


\section{ABSTRACT}

In order to adjust to changing environmental conditions, bacteria use nucleotide second messengers to transduce external signals and translate them into a specific cellular response. Cyclic di-adenosine monophosphate (c-di-AMP) is the only known essential nucleotide second messenger. In addition to the well-established role of this second messenger in the control of potassium homeostasis, we observed that glutamate is as toxic as potassium for a c-di-AMP-free strain of the Gram-positive model bacterium Bacillus subtilis. In this work, we isolated suppressor mutants that allow growth of a c-di-AMP-free strain under these toxic conditions. Characterization of glutamate resistant suppressors revealed that they contain pairs of mutations, in most cases affecting glutamate and potassium homeostasis. Among these mutations, several independent mutations affected a novel glutamate transporter, AimA (Amino acid importer $\underline{\mathbf{A}}$, formerly YbeC). This protein is the major transporter for glutamate and serine in B. subtilis. Unexpectedly, some of the isolated suppressor mutants could suppress glutamate toxicity by a combination of mutations that affect phospholipid biosynthesis and a specific gain-of-function mutation of a mechanosensitive channel of small conductance $(\mathrm{YfkC})$ suggesting the acquisition of a device for glutamate export. Cultivation of the c-di-AMP-free strain on complex medium was an even greater challenge because the amounts of potassium, glutamate, and other osmolytes are substantially higher than in minimal medium. Suppressor mutants viable on complex medium could only be isolated under anaerobic conditions if one of the two c-di-AMP receptor proteins, DarA or DarB, was absent. Also on complex medium, potassium and osmolyte toxicity are the major bottlenecks for the growth of $B$. subtilis in the absence of c-di-AMP. Our results indicate that the essentiality of c-di-AMP in B. subtilis is caused by the global impact of the second messenger nucleotide on different aspects of cellular physiology.

\section{AUTHOR SUMMARY}

Bacteria are exposed to constantly changing environmental conditions. In order to respond to these changes, they use nucleotide second messengers to transduce external signals and translate them into a specific cellular response. Among the repertoire of bacterial second messenger nucleotides, cyclic di-AMP (c-di-AMP) stands out as it is the only second messenger that is essential for the bacteria that produce it, including the Gram-positive model organism Bacillus subtilis. c-di-AMP plays a major role in the control of potassium homeostasis, and we found that glutamate is toxic to a $B$. subtilis strain lacking c-di-AMP to the same extent as potassium. These toxic conditions were the starting point for an extensive suppressor analysis, which led to the identification of a novel glutamate transporter (AimA). If the B. subtilis strain lacking c-di-AMP was cultivated on complex medium, the isolation of suppressor mutants was only possible under anaerobic conditions and if either of the two c-di-AMP- 
binding signal transduction proteins was absent. This suggests that these proteins are a major burden for the cell on complex medium in their c-di-AMP free state. Our result underline the complexity of c-di-AMP signaling and propose new directions for research.

\section{INTRODUCTION}

To achieve appropriate responses to changing environmental conditions, all organisms use second messengers which are formed in response to an environmental cue. In bacteria, specific mono- and dinucleotides play a particularly important role as second messengers (Gomelsky, 2011). The common nucleotide second messengers cyclic AMP, cyclic di-GMP (c-di-GMP), and guanosine tetra/pentaphosphate ((p)ppGpp) are used to prioritize the utilization of carbon sources and to regulate virulence, to control the choice between sessile and motile lifestyles, and to downregulate essential cellular functions upon amino acid starvation, respectively (Görke and Stülke, 2008; McDonough and Rodriguez, 2012; Hauryliuk et al., 2015; Jenal et al., 2017; Hengge et al., 2019). The more recently discovered second messenger cyclic di-AMP is unique in several respects. First, this nucleotide is essential in many bacteria that produce it, suggesting that this molecule plays a role in central cellular activities. Essentiality of c-di-AMP has been observed in many Firmicutes, such as Bacillus subtilis, Listeria monocytogenes, Streptococcus agalactiae, and Staphylococcus aureus, but also in the genome-reduced bacterium Mycoplasma pneumoniae and in Chlamydia trachomatis (Woodward et al., 2010; Luo and Helmann, 2012; Mehne et al., 2013; Barker et al., 2013; Corrigan et al., 2015; Blötz et al., 2017; Devaux et al., 2018b). Second, c-di-AMP is toxic if it accumulates in the

cells, and in many bacteria this accumulation results in strongly increased stress sensititvity (Smith et al., 2012; Mehne et al., 2013; Huynh et al., 2015; Gundlach et al., 2015b; Zhu et al., 2016; Zarrella et al., 2018). Third, c-di-AMP can bind to proteins and the mRNA molecules encoding them to control both the expression and the activity of certain proteins, as in the case of the $B$. subtilis potassium transporters KtrAB and KimA (Nelson et al., 2013; Gundlach et al., 2017b; Gundlach et al., 2017a; Gundlach et al., 2019). Last, but not least, c-di-AMP is not intrinsically present in eukaryotic organisms, and the presence of the molecule is an indication for infection with pathogenic bacteria. Indeed, c-di-AMP is recognized by the human immune system. It binds the STING protein thus resulting the induction of an innate immune response (Woodward et al., 2010).

The essentiality and toxicity of c-di-AMP for many bacteria have induced a great interest in understanding the reasons behind it. Classically, genes were regarded to be essential if they were absolutely needed for growth under optimal growth conditions, typically on complex medium at $37^{\circ} \mathrm{C}$ (Hutchison, 1999; Commichau et al., 2013; Rancati et al., 2018). However, many genes are not described as being essential because there may be paralogues that encode very similar functions as 
has been described for the c-di-AMP producing diadenylate cyclases in B. subtilis (Luo and Helmann, 2012; Mehne et al., 2013). In some cases, one essential function can be carried out by very different proteins, and such genes are essential only under specific conditions, i. e. the absence of amino acids from the medium makes the corresponding biosynthetic genes essential. These genes have been called conditional essential genes. Finally, some genes may not be essential but their loss would cause a substantial decrease of fitness for the cells, resulting either in the disappearance of such mutants or in the acquisition of suppressor mutations. Such genes or proteins have been designated quasi-essential, and the effect of their loss has been studied for the B. subtilis DNA topoisomerase TopA, the endoribonuclease RNase Y, and the modulator of lipid biosynthesis, YqhY (Tödter et al., 2017; Reuß et al., 2019; Benda et al., 2020). Very recently, essential processes have been ranked on a global scale for the first time by the determination of the speed of death of mutants (Gallagher et al., 2020).

Two lines of research have provided important insights into the reasons of c-di-AMP essentiality. Studies to identify target molecules that interact with c-di-AMP demonstrated that many proteins involved in potassium and osmolyte homeostasis - both transporters and their regulators are controlled by c-di-AMP to limit the intracellular concentrations of potassium and osmotically compatible compounds to physiologically reasonable levels (Corrigan et al., 2013; Corrigan et al., 2015; Moscoso et al., 2016; Schuster et al., 2016; Huynh et al., 2016; Rubin et al., 2018; Devaux et al., 2018b; Pham et al., 2018; Quintana et al., 2019; Gundlach et al., 2019; Latoscha et al., 2020). In a complementary approach, suppressor mutants of bacterial strains that either lack or accumulate c-di-AMP have been studied. These analyses revealed that c-di-AMP is conditionally essential in L. monocytogenes, S. aureus and B. subtilis. While the nucleotide is required for growth on complex media, it is dispensable on minimal media (Whiteley et al., 2015; Gundlach et al., 2017b; Zeden et al., 2018). For a B. subtilis mutant lacking c-di-AMP, growth was only possible on minimal medium as long as the potassium concentration in the medium is low and glutamate is not present (Gundlach et al., 2017b; Krüger et al., 2020b). On complex medium, the lack of c-di-AMP leads to an accumulation of (p)ppGpp (Whiteley et al., 2015), the second messenger that results in a global reprogramming of transcription, translation, and metabolism upon amino acid starvation. Inactivation of the enzymes that produce (p)ppGpp restores the viability of the strain lacking c-di-AMP on complex medium. The accumulation of (p)ppGpp leads to the inability of the transcription factor CodY to bind to its targets and thus in deregulation of a large regulon that includes many genes for osmolyte uptake and amino acid metabolism, and this uncontrolled expression of the CodY regulon is responsible for the essentiality of c-di-AMP for L. monocytogenes on complex media (Whiteley et al., 2015). In S. aureus, c-di-AMP is dispensable for growth on complex medium under anaerobic conditions or when the bacteria acquire suppressor mutations that affect osmolyte or glutamate uptake (Zeden et al., 2018; 
Krüger et al., 2020b; Zeden et al., 2020). Both types of studies support the idea that the control of potassium and osmolyte homeostasis is the central essential function of c-di-AMP in many bacteria that produce it (Commichau et al., 2018a; He et al., 2020; Stülke and Krüger, 2020).

We are interested in c-di-AMP signaling in the model organism B. subtilis. In contrast to S. aureus or L. monocytogenes, B. subtilis encodes three diadenylate cyclases that produce c-di-AMP, the constitutively expressed enzymes CdaA and DisA, and the sporulation-specific enzyme CdaS (Mehne et al., 2013; Mehne et al., 2014). Mutants lacking both the $c d a A$ and disA genes that encode the constitutive enzymes are not viable on complex medium and tolerate only very low potassium concentrations and no glutamate in minimal medium. In B. subtilis, c-di-AMP binds to two signal transduction proteins, DarA and DarB, to several potassium, osmolyte and magnesium transporters, as well as to the riboswitch that controls the expression of the high affinity potassium transporters KtrAB and KimA (Nelson et al., 2013; Gundlach et al., 2015a; Gundlach et al., 2019).

To get a better understanding of the global role of c-di-AMP for the physiology of $B$. subtilis, we performed extensive suppressor analysis of strains lacking c-di-AMP that had adapted to tolerate the presence of glutamate or that are viable on complex medium. These suppressor analyses were complemented by a transcriptome analysis to understand the roles of glutamate and potassium as well as of c-di-AMP for global gene expression in B. subtilis. Our results indicate that the adaptation to either glutamate or complex medium usually requires the simultaneous presence of multiple suppressor mutations that often affect potassium uptake, osmolyte uptake, or glutamate metabolism. Moreover, a mechanosensitive channel and phospholipid biosynthesis play an important role in the sensitivity of a strain lacking c-di-AMP to glutamate. Strikingly, this study identified the amino acid transporter YbeC (renamed AimA, amino acid importer $\underline{A}$ ) as the major glutamate transporter in B. subtilis. On complex medium, the c-di-AMP-binding signal transduction proteins DarA and DarB seem to be toxic in the absence of c-di-AMP, and only in their absence we could isolate suppressor mutants. The obtained results shed light on the complexicity of c-di-AMP essentiality and open new directions of research.

\section{RESULTS}

\section{Growth of a strain lacking c-di-AMP in the presence of glutamate requires distinct combinations of suppressor mutations}

A B. subtilis strain lacking all three diadenylate cyclases is unable to grow at potassium concentrations of $5 \mathrm{mM}$ or higher or in the presence of glutamate (even at low potassium concentrations) (Gundlach et al., 2017b; Krüger et al., 2020b). In order to get more comprehensive insights into the toxicity of glutamate for the c-di-AMP-free strain GP2222 (designated $\Delta$ dac from here on), we decided to isolate 
and study a larger set of suppressor mutants that tolerate the presence of glutamate. For this purpose, we plated the $\Delta d a c$ strain (Gundlach et al., 2017b) on MSSM medium containing glutamate $(1 \% \mathrm{w} / \mathrm{v})$ and a low concentration of potassium $(0.1 \mathrm{mM} \mathrm{KCl})$. While suppressor mutants appear readily after overnight cultivation when potassium toxicity was the growth-limiting problem (Gundlach et al., 2017b), the toxicity of glutamate could only be overcome after several days. Moreover, the number of suppressor mutants was strongly reduced as compared to selection in the presence of potassium and ammonium as the nitrogen source. After four days at $37^{\circ} \mathrm{C}, 14$ colonies could be isolated (see Fig. 5.1, $\Delta d a c)$. These strains were subjected to whole genome sequencing to identify the responsible mutations (Table 5.1). The long time for suppressor mutations to appear can be explained by each mutant harboring multiple mutations. In addition, this explains why the overall number of suppressors was rather low.

Table 5.1. Characterization of suppressor mutants derived from the $\Delta$ dac strain GP2222 in MSSM minimal medium with glutamate and $0.1 \mathrm{mM} \mathrm{KCl}{ }^{1}$

\begin{tabular}{|c|c|c|c|c|c|c|}
\hline Strain & Genotype & $\begin{array}{l}\text { Ammonium, } \\
0.1 \mathrm{mM} \mathrm{KCl}\end{array}$ & $\begin{array}{l}\text { Ammonium, } \\
5 \mathrm{mM} \mathrm{KCl}\end{array}$ & $\begin{array}{l}\text { Glutamate, } \\
0.1 \mathrm{mM} \mathrm{KCl}\end{array}$ & $\begin{array}{l}\text { Glutamate, } \\
5 \mathrm{mM} \mathrm{KCl}\end{array}$ & LB \\
\hline \multirow[t]{2}{*}{ GP2840 } & $k \operatorname{trC}(\mathrm{fs}) p / s C-\mathrm{A} 61 \mathrm{~V} n d h F(\mathrm{fs})$ & & & & & \\
\hline & $\operatorname{sig} D-G 88 D$ & +++ & +++ & +++ & +++ & - \\
\hline \multirow[t]{3}{*}{ GP3079 } & $\Delta(a b h-k i n C-y k q A-k \operatorname{tr} C) p l s C-$ & & & & & \\
\hline & L48P yfkC-R42W fliP (fs) hbs- & ++ & +++ & +++ & +++ & + \\
\hline & I32T ndoA-G6S & & & & & \\
\hline \multirow[t]{2}{*}{ GP3077 } & $k \operatorname{trC}(\mathrm{fs}) \operatorname{aimA} / g l p Q$ (trunc) & & & & & \\
\hline & $y b c c(\mathrm{fs})$ & +++ & +++ & +++ & +++ & - \\
\hline \multirow[t]{2}{*}{ GP2841 } & $\operatorname{gudB} B^{+} \operatorname{accC}-\mathrm{H} 298 \mathrm{Y} f l i G(\mathrm{fs})$ & & & & & \\
\hline & & + & + & ++ & + & - \\
\hline \multirow[t]{2}{*}{ GP2842 } & nhak-S187F aimA (fs) & & & & & \\
\hline & & +++ & +++ & ++ & + & - \\
\hline \multirow[t]{2}{*}{ GP3078 } & plsC-A61V aimA-T374M & & & & & \\
\hline & ktrB-G313D sigD-G88D & +++ & + & +++ & - & - \\
\hline \multirow[t]{2}{*}{ GP2849 } & $y b c C-K 722 \mathrm{~L}, 1724 \mathrm{~F} \operatorname{aimA}(\mathrm{fs})$ & & & & & \\
\hline & & +++ & +++ & +++ & +++ & - \\
\hline \multirow[t]{2}{*}{ GP2837 } & plsC-L48P yfkC-R42W fliP (fs) & & & & & ++ \\
\hline & & +++ & +++ & +++ & +++ & + \\
\hline \multirow[t]{2}{*}{ GP3099 } & plsC-L48P yfkC-R42W fliP (fs) & & & & & \\
\hline & $\cot O-134 \mathrm{~F}$ & +++ & + & +++ & - & - \\
\hline
\end{tabular}




\begin{tabular}{|c|c|c|c|c|c|c|}
\hline \multirow[t]{2}{*}{ GP3464 } & \multicolumn{6}{|l|}{ plsC-P110S flhA (fs) } \\
\hline & & ++ & ++ & ++ & - & ++ \\
\hline \multirow[t]{2}{*}{ GP2224 } & yqeG $(\mathrm{fs}) \operatorname{aim} A(\mathrm{fs})$ yqeV $(\mathrm{fs})$ & & & & & \\
\hline & & +++ & +++ & +++ & +++ & - \\
\hline \multirow[t]{3}{*}{ GP2838 } & ndhF-V237E, M241L, H242L, & & & & & \\
\hline & A243D, G244C, L245F & +++ & ++ & ++ & ++ & ++ \\
\hline & $\Delta \mathrm{ICEBs} 1$ (int-yddM) yoal (fs) & & & & & \\
\hline \multirow[t]{2}{*}{ GP3473 } & $n d h F(\mathrm{fs}) \operatorname{aim} A$ (fs) gudB $B^{+}$fliH & & & & & \\
\hline & (fs) yuiE-G37V & ++ & ++ & ++ & - & - \\
\hline \multirow[t]{2}{*}{ GP3474 } & $n d h F(\mathrm{fs}) \operatorname{aimA}(\mathrm{fs}) y k p A(\mathrm{fs})$ & & & & & \\
\hline & $k \operatorname{trC}(\mathrm{fs})$ flik (fs) & ++ & +++ & +++ & +++ & - \\
\hline \multirow[t]{2}{*}{ GP2839 } & flik (fs) rpoB-Q109P rbsB- & & & & & \\
\hline & $\mathrm{R} 175 \mathrm{~L}$ & ++ & ++ & ++ & - & - \\
\hline
\end{tabular}

${ }^{1}$ Growth on different media was judged after plating on corresponding agar plates.

${ }^{2}$ This strain was isolated in a previous study (Krüger et al., 2020b).

As observed previously (Krüger et al., 2020b), three strains were affected in ktrC. In one of these strains (GP3079), the genomic region from abh to $k \operatorname{trC}$ was deleted resulting in reduced uptake of potassium via KtrCD. Similarly, strain GP3078 has a mutation affecting the high-affinity potassium transporter KtrB, and GP2842 also carries a mutation (nhaK) that affects potassium homeostasis. Interestingly, the same mutation (NhaK-S187F) had been isolated in a suppressor screen of the $\Delta d a c$ strain in the presence of $5 \mathrm{mM}$ potassium (Gundlach et al., 2017b) indicating that the mutant protein has an increased activity in potassium export.

As mentioned above, the suppressor strains usually had multiple mutations. Since we never experienced the systematic acquisition of multiple mutations in prior suppressor screens (Gundlach et al., 2015b; Rosenberg et al., 2016; Kampf et al., 2018; Reuß et al., 2019), it seems likely that more than one mutation is required to achieve the suppression. Since the selective pressure in our experiment was directed towards tolerating otherwise toxic glutamate, we expected that the suppressor mutants would contain mutations affecting glutamate metabolism. Indeed, the á priori cryptic glutamate dehydrogenase was decryptified by the deletion of nine base pairs in two mutants (GP2841 and GP3473), thus allowing glutamate degradation (Belitsky and Sonenshein, 1998; Commichau et al., 2008). Moreover, seven independently isolated mutants carried mutations affecting an amino acid transporter, YbeC. This protein has recently been identified as the major serine transporter of B. subtilis (Klewing et al., 2020), and it is tempting to speculate that $\mathrm{YbeC}$ is the major glutamate 
transporter as well (see below). However, seven of the isolated mutants did not carry any mutation that is easily linked to glutamate homeostasis. The previously reported suppressor mutant GP2840 had mutations affecting PIsC, NdhF, and SigD (acyl-ACP:1-acylglycerolphosphate acyltransferase, NADH dehydrogenase, Sigma factor $\sigma^{D}$, respectively) in addition to the $k \operatorname{tr} C$ mutation (Krüger et al., 2020b). Strikingly, $p l s C$ and $n d h F$ are affected in six and four different suppressor mutants respectively. Several mutants carried mutations affecting motility: In addition to two sigD mutants, seven additional mutants carried mutations in flagellar genes that on one hand depend on a functional SigD for their expression, and on the other hand are required for SigD activity (Calvo and Kearns, 2015). In addition, three of the 14 different independent suppressor mutants carry the same point mutation in the $y f k C$ gene, encoding a mechanosensitive channel that releases ions from the cell (Wahome and Setlow, 2007; Hoffmann et al., 2008). This mutation results in a substitution of Arg-42 by a Trp residue.

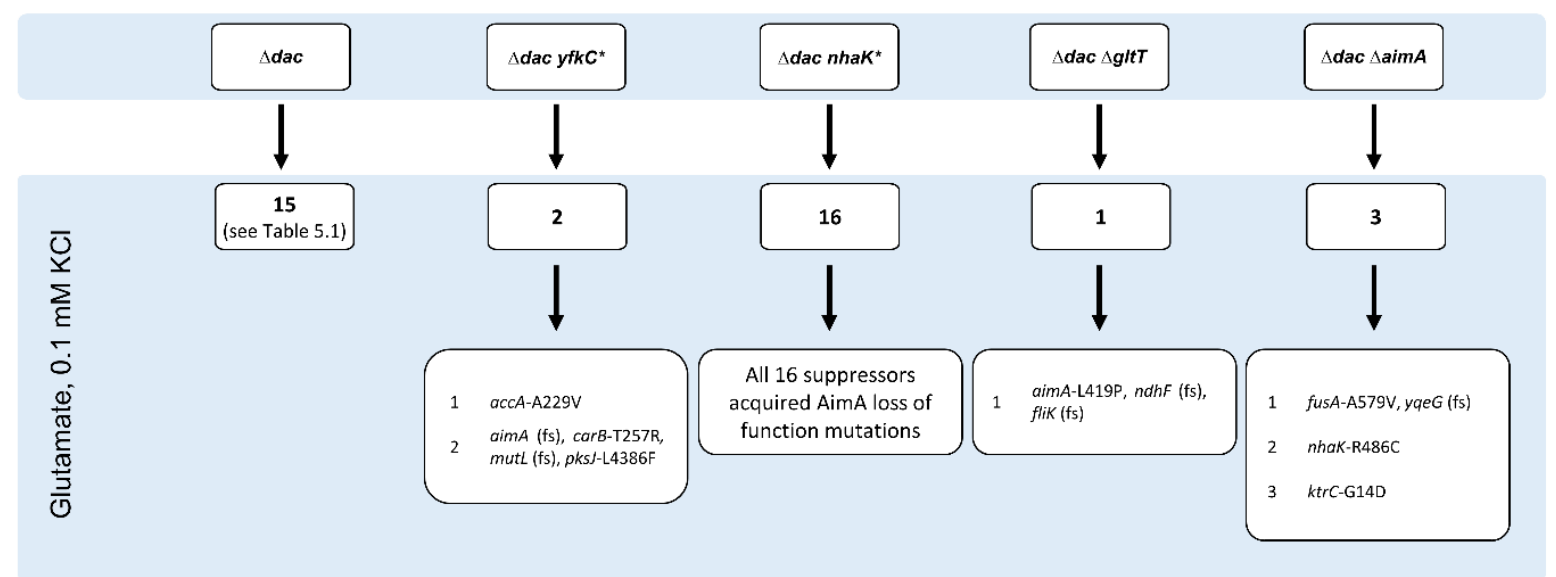

Fig. 5.1. A suppressor screen with a strain lacking c-di-AMP reveals the complexity of glutamate toxicity.

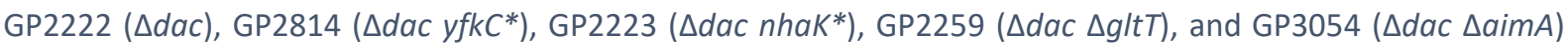
were cultivated in MSSM medium with glutamate and $0.1 \mathrm{mM} \mathrm{KCl}$ and suppressors were isolated and analyzed by whole genome sequencing. Note that for GP2222 ( $\Delta d a c), 14$ mutants were isolated in this study and one mutant was isolated previously (Krüger et al., 2020b) but studied in depth here, thus resulting in a total number of 15 mutants.

We then tested the ability of the suppressor mutants to grow on minimal medium at low and high potassium concentrations with ammonium or glutamate as the nitrogen source. Moreover, the ability to grow on a complex medium was tested (see Table 5.1, S5.1 Fig.). Since the mutants were isolated in the presence of glutamate and $0.1 \mathrm{mM}$ potassium, it is not surprising that all strains grew on the corresponding medium. Moreover, most of the strains were viable with ammonium as the single nitrogen source irrespective of the potassium concentration. Most of the mutants were also capable of growing at the elevated potassium concentration in the presence of glutamate. However, only four 
of the 15 tested strains were viable on complex medium. Two of them grew well on LB and these strains had the identical combination of mutations in $y f k C$ and $p l s C$ (see Table 5.1, see below). This suggests that the adaptation to complex medium requires more than resistance to potassium and glutamate.

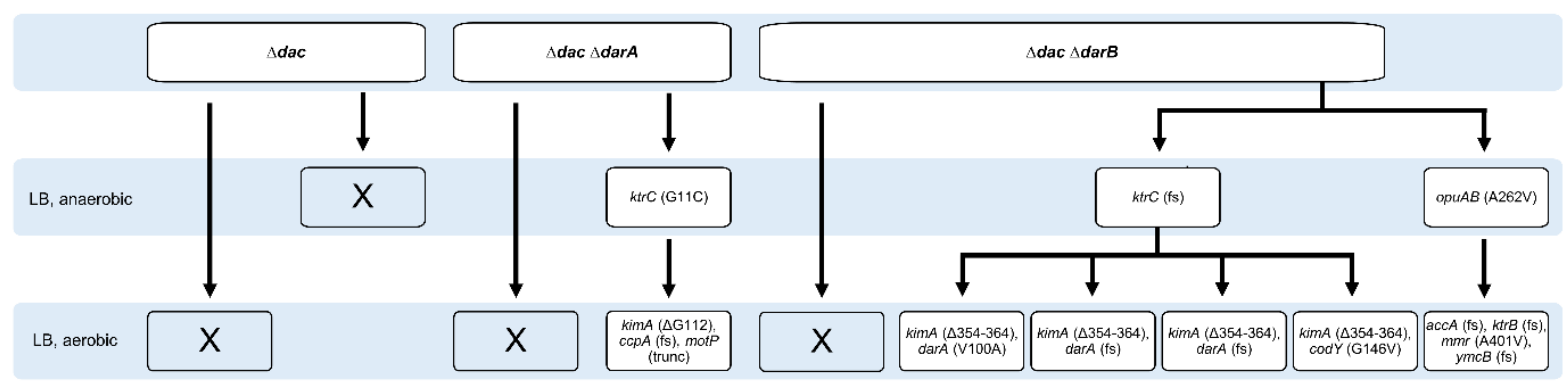

Fig. 5.2. A suppressor screen with a strain lacking c-di-AMP on complex medium. GP2222 ( $\triangle$ dac), GP2420 ( $\triangle$ dac $\Delta$ darA), or GP2779 ( $\Delta$ dac $\Delta$ darB) were cultivated on LB agar plates and incubated under aerobic or anaerobic conditions as indicated. The table shows how the parental strain evolved, and under which conditions suppressors could be isolated. The suppressor mutants were analyzed by whole genome sequencing.

\section{Stepwise adaptation of a strain lacking c-di-AMP to growth on complex medium}

Most of the suppressor mutants that tolerate the presence of glutamate and potassium were unable to grow on complex medium under standard laboratory conditions. The standard definition of essentiality refers to optimal growth conditions, i. e. to a complex medium and growth at $37^{\circ} \mathrm{C}$ in the presence of oxygen for B. subtilis (Commichau et al., 2013; Rancati et al., 2018). This prompted us to investigate the growth requirements of the $\Delta d a c$ mutant GP2222 more rigorously. First, we attempted to isolate suppressor mutants derived from GP2222 under standard conditions as defined above. However, we never succeeded in isolating a single suppressor mutant. A recent study on c-di-AMP essentiality in S. aureus revealed that the nucleotide is dispensable under anaerobic conditions (Zeden et al., 2018). Therefore, we tried to isolate suppressor mutants of GP2222 in the absence of oxygen. While the wild type strain $B$. subtilis 168 grew under these conditions, again no suppressor mutants of the $\Delta d a c$ strain could be isolated (see Fig. 5.2).

In B. subtilis, c-di-AMP binds two signal transduction proteins, DarA and DarB (Gundlach et al., 2015a; Gundlach et al., 2019). In a suppressor screen using a L. monocytogenes strain lacking c-di-AMP, several mutations in the corresponding genes were observed (Whiteley et al., 2015). We therefore constructed strains that lack both c-di-AMP and either the darA or the darB gene, and used these strains to isolate suppressors on complex medium under aerobic and anaerobic conditions. Again, no suppressors could be obtained under aerobic conditions; however, we detected one and two 
suppressors for the $\Delta d a c \Delta d a r A$ and $\Delta d a c \Delta d a r B$ mutants, respectively, under anaerobic conditions (see Fig. 5.2). Whole genome sequencing revealed that the mutation present in the $\Delta$ dac $\Delta$ darA mutant changed Gly-11 of KtrC to a cysteine residue. The suppressor mutants derived from the $\Delta$ dac $\Delta$ dar $B$ mutant had a frameshift in $k t r C$ resulting in the expression of a truncated $\mathrm{KtrC}$ protein or a mutation affecting the permease domain OpuAB of the OpuA glycine betaine transporter (Kempf and Bremer, 1995), respectively (Fig. 5.2). These results strongly support the idea that the accumulation of potassium or of osmoprotective compatible solutes is the reason for the essentiality of c-di-AMP (Whiteley et al., 2015; Commichau et al., 2018a; Zeden et al., 2018).

An initial characterization of these suppressor mutants revealed that they were viable under anaerobic conditions, but not in the presence of oxygen. Therefore, we used the three strains for a further round of suppressor isolation. Indeed, we were able to obtain suppressors that tolerate the presence of oxygen for all three mutants (Fig. 5.2, S5.3 Fig.). Whole-genome sequencing revealed that the initial suppressors with defective $\mathrm{KtrC}$ proteins had all acquired very specific mutations in the kimA gene, encoding the major high-affinity potassium transporter KimA. In the mutant isolated from the darA ktrC mutant, the absolutely conserved glycine in transmembrane helix 3 of KimA was deleted. Helix 3 is essential for potassium binding of KimA (Tascón et al., 2020). All four independent suppressors isolated from the $\Delta$ dac $\Delta$ darB $k \operatorname{trC}$ mutant had a deletion of 11 amino acids in the transmembrane helix 9 . Based on the high conservation of the deleted residues, we conclude that the mutant KimA proteins were inactive or less active than the wild type protein. Again, the kimA suppressor mutations were accompanied by other mutations (see Fig. 5.2). In the suppressor mutant GP3010 derived from the $\Delta$ dac $\triangle$ darA $k \operatorname{trC}$ mutant, we found a $155 \mathrm{bp}$ deletion that results in the truncation the global transcription regulator for catabolic genes and operons, CcpA, and in the conversion of the start codon of motP, encoding the $\mathrm{Na}^{+}$-coupled MotP flagellar stator protein to a stop codon. For the $\Delta d a c \Delta d a r B k t r C$ suppressor mutant, two aerobic suppressors were subjected to whole genome sequencing. In one strain (GP2845), we found a point mutation in the darA gene that results in a substitution of the conserved Val-100 residue by an alanine in the DarA protein. The second suppressor (GP3094) carried mutations affecting the CodY transcription factor (G146V substitution) and the unknown SP $\beta$ phage protein YonD (D255H). In two additional suppressor mutants, we found identical frameshift mutations in darA that result in a truncation of the DarA protein after amino acid 64. Finally, we also characterized an aerobic suppressor (GP2847) derived from the anaerobic $\Delta d a c$ $\triangle d a r B$ opu $A B$ suppressor mutant. In this case, again a potassium channel subunit (KtrB) was inactivated as the result of a frameshift mutation. Moreover, this strain carried a frameshift mutation in the essential accA gene encoding a subunit of acetyl-CoA carboxylase that resulted in the formation of a truncation after amino acid 108 and mutations in the genes encoding the putative methylenomycin $\mathrm{A}$ 
exporter Mmr and the tRNA methylthiotransferase YmcB. These results again highlight the importance of potassium homeostasis for the adaptation of strains lacking c-di-AMP.

Taken together, a B. subtilis strain lacking c-di-AMP is only able to grow under standard laboratory conditions if it acquires several suppressing mutations. The results of the two suppressor screens in the presence of glutamate and directly on complex medium demonstrate a distinct selective pressure under both conditions resulting in different sets of mutations that allow aerobic growth on complex medium. The importance of the initial loss of the c-di-AMP receptor proteins DarA and DarB for the direct adaptation to complex medium demonstrates that these proteins interfere with the growth of $B$. subtilis if they are present in the ligand-free apo-state. Indeed, apo-DarB was recently shown to trigger growth-inhibitory (p)ppGpp synthesis by the Rel protein (Krüger et al., 2020c; Peterson et al., 2020). For DarA, this is the first time that a phenotype for the corresponding mutant is reported. The repeated isolation of mutations affecting potassium uptake even in iterative cycles of suppressor screens highlights the severe toxicity of potassium for B. subtilis in the absence of c-di-AMP.

\section{A global transcription study of the regulatory effects of potassium, glutamate, and c-di-AMP}

In order to obtain deeper insights into the physiological role of c-di-AMP, we decided to study the impact of a lack of this second messenger on global gene expression by a transcriptome analysis as a complementary approach to the suppressor analysis. Since c-di-AMP has been implicated in the interplay of potassium and glutamate homeostasis, we analysed the global gene expression for the wild type strain B. subtilis 168 at low $(0.1 \mathrm{mM})$ and high $(5 \mathrm{mM})$ potassium concentrations and in the presence of ammonium and glutamate as the nitrogen source (S5.1 Supporting Information). Moreover, we used the c-di-AMP free strain ( $\Delta d a c)$ GP2222 as well as the isogenic suppressor mutant GP2223 that is able to grow at $5 \mathrm{mM}$ potassium (see Fig. 5.3 for the regulatory network and S5.1 Table). Strikingly, expression of several genes involved in fermentation and respiration was strongly reduced in the absence of c-di-AMP. The Idh-IctP and $c y d A B C D$ operons encoding lactate dehydrogenase and the lactate exporter as well as the terminal quinole oxidase, are repressed by the NADH-responsive transcription factor Rex (Larsson et al., 2005; Gyan et al., 2006). The about 100-fold repression of these genes in the absence of c-di-AMP suggests that the NADH levels are reduced in the mutant. This conclusion is also supported by the increased expression (seven-fold) of the $n d h F-y b c C$ operon (see S5.1 Table) which codes for a NADH dehydrogenase and thus also contributes to the oxidation of $\mathrm{NADH}_{2}$ to NAD (Qin et al., 2019). Importantly, the $n d h F$ gene and its downstream gene ybcC were affected in several of the suppressor mutants suggesting that these mutations counteract the increased expression of the conserved $n d h F-y b c C$ operon. In addition to genes of the Rex regulon, genes of the SigO regulon that are involved in the response to acid stress (MacLellan et al., 2008) and 
several competence genes were severely reduced in the $\Delta d a c$ mutant GP2222. The strong reduction of competence gene expression in response to the absence of c-di-AMP suggests that this second messenger might be required for genetic competence and transformation in B. subtilis. To test this hypothesis, we attempted transformation of strain GP2222 using chromosomal DNA. No transformants were obtained, whereas the control strain 168 was efficiently transformable. Thus, the lack of c-di-AMP results in a loss of genetic competence as a result of reduced competence gene expression.

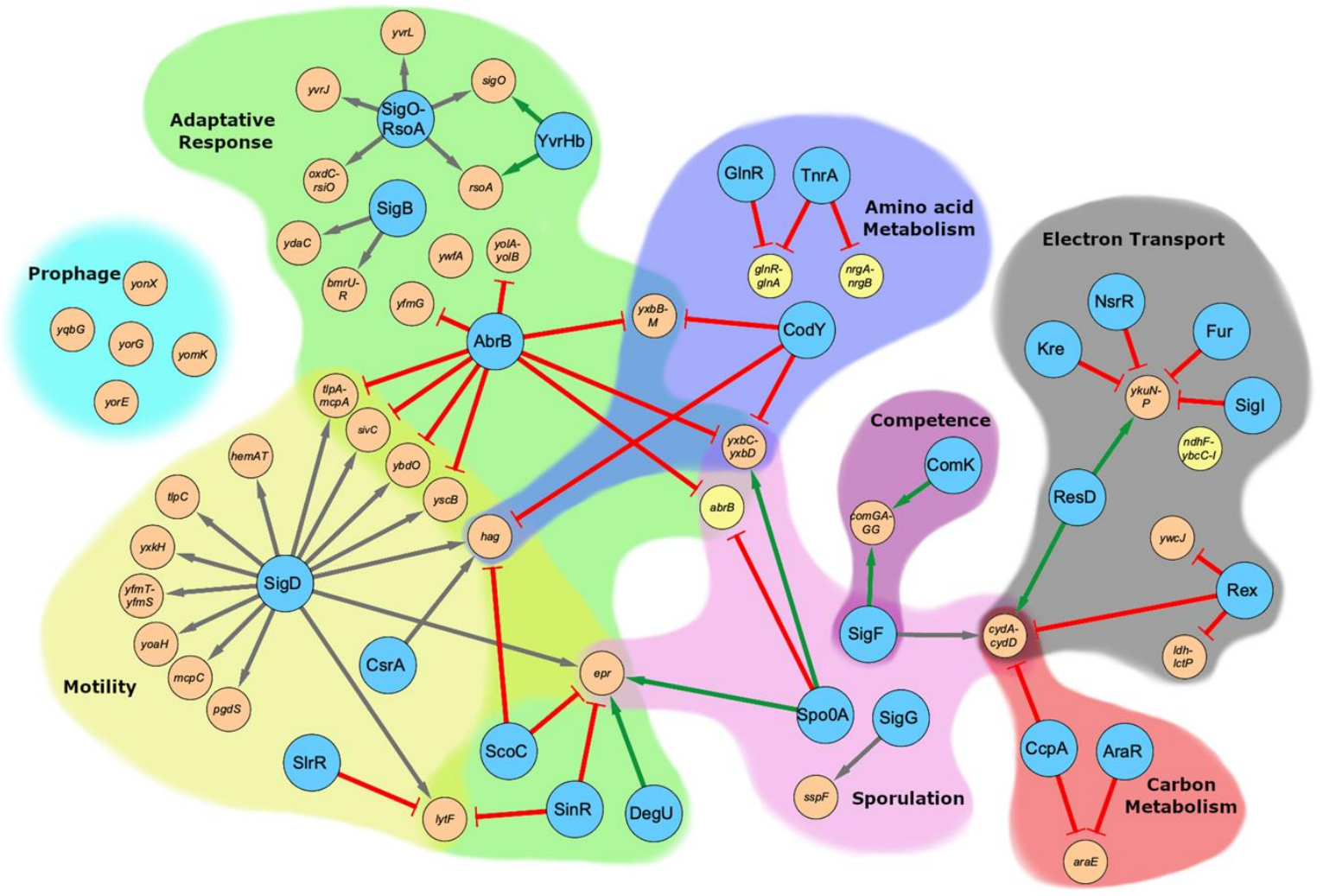

Fig. 5.3. Regulatory network of the most strongly up- and downregulated genes as a result of the lack of c-di-AMP. Expression ratios of $B$. subtilis wild type and GP2222 ( $\Delta d a c$ ) with ammonium and $0.1 \mathrm{mM} \mathrm{KCl}$ were calculated and the most up- or downregulated genes were clustered into operons, regulons, and grouped depending on their physiological function. Regulators are depicted as large blue nodes, and regulated operons or genes as yellow and orange nodes for up- and downregulated genes, respectively). Arrows: green: activation; gray: sigma-factor; red: repression.

It has been shown before that the expression of the high affinity potassium uptake systems KtrAB and KimA is repressed in the presence of potassium via a c-di-AMP sensitive riboswitch (Nelson et al., 2013; Gundlach et al., 2017b; Gundlach et al., 2017a). In agreement with those results, these genes were most strongly repressed by potassium in our analysis in the wild type strain 168 . The expression of the kimA transporter gene was 112 - and 17 -fold repressed at $5 \mathrm{mM}$ potassium in the 
presence of glutamate or ammonium, respectively; whereas the expression of the ktrAB channel operon was reduced 29- and 4-fold, respectively (see S5.2 Table). As expected, the expression of both kimA and ktrAB is increased in the absence of c-di-AMP; however, both genes are still repressed by potassium (eight- and five-fold, respectively), indicating a second, c-di-AMP independent mechanism of transcriptional regulation of these potassium transport systems. The particular importance of potassium for the cell and for KimA as a major high-affinity transporter is highlighted by the fact that the $\operatorname{kimA}$ gene belongs to the most strongly expressed genes at $0.1 \mathrm{mM}$ potassium (position \#50 in the presence of glutamate), and that it is even the most strongly expressed transporter in $B$. subtilis under these conditions (see https://www.ncbi.nlm.nih.gov/geo/query/acc.cgi?acc=GSE156738 for the full data set).

Taken together, the global transcription analysis supported the idea that c-di-AMP is involved in multiple cellular functions. Moreover, the analysis confirmed a particular role of genes involved in controlling NADH homeostasis, as already suggested by the suppressor analysis and by the differential effects of aerobic and anaerobic conditions to allow suppressor formation of the strains lacking c-di-AMP.

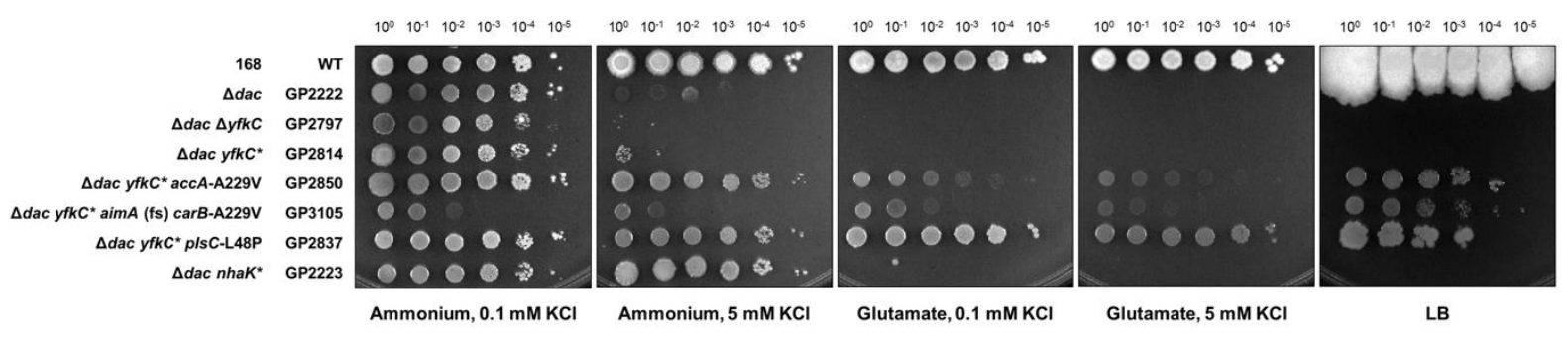

Fig. 5.4. The influence of the mechanosensitive channel $\mathrm{YfkC}$ on growth of a $B$. subtilis strain lacking C-di-AMP in the presence of glutamate. $B$. subtilis strains were cultivated in MSSM minimal medium with $0.1 \mathrm{mM} \mathrm{KCl}$ and glutamate. Cells were harvested, washed, and the $\mathrm{OD}_{600}$ was adjusted to 1.0. Serial dilutions were dropped onto MSSM minimal plates with the indicated potassium concentration and ammonium or glutamate, or on LB plates.

\section{The mechanosensitive channel YfkC contributes to the glutamate sensitivity of a c-di-AMP free strain} Three of the suppressor mutants isolated in the presence of glutamate carry mutations affecting the mechanosensitive channel of small conductance, YfkC (see Table 5.1). Strikingly, all three independently isolated mutants carry precisely the same mutation that results in a R42W substitution in the protein (see S5.4 Fig.). This residue is located at the end of the first trans-membrane helix (Zhang et al., 2012) and the mutation may affect the relative positioning of the trans-membrane helices 1 and 2. Another striking feature is that this mutation is in all three cases accompanied by point mutations in the $p / s C$ gene (see below). The presence of the same amino acid substitution in YfkC in all three 
strains suggested that this mutation resulted in a gain of function rather than in a loss of $\mathrm{YfkC}$ activity (from here on, we designate the mutant allele $y f k C^{*}$, the protein $\mathrm{YfkC}^{*}$ ). To address this question, we constructed $\Delta d a c \Delta y f k C$ and $\Delta d a c y f k C^{*}$ strains lacking all diadenylate cyclases and assayed the growth of these strains on minimal medium containing glutamate or ammonium as the nitrogen source at high and low potassium concentrations (see Fig. 5.4). Both strains were viable when cultivated on plates containing ammonium and $0.1 \mathrm{mM}$ potassium whereas they were unable to grow in the presence of glutamate or at the high potassium concentration. These observations indicate that neither the deletion of the $y f k C$ gene nor $y f k C^{*}$ mutation alone were sufficient to allow suppression of the glutamate and potassium toxicity of the $\Delta d a c$ mutant. To address this issue further, we attempted the isolation of suppressor mutants from the $\Delta d a c$ strain either lacking $y f k C$ or carrying the $y f k C^{*}$ allele in the presence of glutamate. No suppressor mutants could be isolated from the $\Delta d a c \Delta y f k C$ mutant, whereas two suppressor mutants were obtained from the $\Delta d a c y f k C^{*}$ mutant (see Fig. 5.1). This observation supports the idea that the specific $\mathrm{R} 42 \mathrm{~W}$ substitution of $\mathrm{YfkC}^{*}$ is a prerequisite for the adaptation of the c-di-AMP-free strain to growth in the presence of glutamate. Whole genome sequencing of the two glutamate-resistant suppressor mutants identified a mutation in accA in strain GP2850 (see below). This mutation supports the requirement of paired mutations affecting $y f k C$ and lipid biosynthesis. The second suppressor strain, GP3105, carries mutations affecting the amino acid transporter YbeC, the CarB subunit of the carbamoyl-phosphate synthetase, the endonuclease MutL and the polyketide synthase PksJ. Among these mutations, the frameshift in the $y b e C$ gene is likely the most relevant (see below). These suppressor mutants were also tested for their growth in the presence of glutamate or increased potassium concentrations (see Fig. 5.4). Both were viable in the presence of glutamate irrespective of the potassium concentration. However, the growth in the presence of glutamate was much better in the case of the yfkC* plsC (L48P) mutant GP2837 (Fig. 5.4). Taken together, these results suggest that the $y f k C^{*}$ mutation in combination with the lipid biosynthetic or ybec mutations is rather specific for the protection of the $\Delta d a c$ mutant from glutamate toxicity. In the growth assay, we also included the $\Delta \operatorname{dac}$ nhaK (S187F) mutant that has been isolated for resistance to potassium (Gundlach et al., 2017b). As expected, this strain grew at $5 \mathrm{mM}$ potassium but was as sensitive to glutamate as the original $\Delta d a c$ mutant, however we observed the rapid formation of suppressor mutants in the presence of glutamate (see Fig. 5.4, see below). As the NhaK allele used in this experiment is thought to exhibit increased potassium export activity (Gundlach et al., 2017b), it is tempting to speculate the mutant mechanosensitive channel $\mathrm{YfkC}^{*}$ is able to export glutamate to overcome the toxicity of this amino acid. 


\section{A reduction of phospholipid biosynthesis helps to overcome the lack of c-di-AMP}

The analysis of suppressor mutants that were viable in the presence of glutamate or on complex medium identified several mutations that affect proteins involved in phospholipid biosynthesis, i.e. AccA, AccC, and PIsC (see Fig. 5.1, Fig. 5.2, Table 5.1). The accA and accC genes encode subunits of the essential acetyl-CoA carboxylase. Unexpectedly, a frameshift had occurred in the accA gene of strain GP2847. Since malonyl-CoA synthesis is the essential first step in fatty acid and phospholipid biosynthesis, it is likely that this mutation can be intrinsically suppressed and thus does not result in a complete loss of AccA synthesis. The accA mutation isolated in the $\triangle d a c y f k C^{*}$ mutant results in an A229V substitution in the active site of the enzyme (see S5.5 Fig.; Bilder et al., 2006; Silvers et al., 2016). For AccC, the mutation (H298Y) is located in the highly conserved active site motif of the enzyme (S5.5 Fig). The localization of these mutations strongly suggests that the initial step of lipid biosynthesis, the formation of malonyl-CoA, is severely impaired in the suppressor mutants.

$\mathrm{PIsC}$ is the acyl-ACP:1-acylglycerolphosphate acyltransferase that catalyzes the formation of phosphatidic acid in the synthesis of phospholipids. Interestingly, the $p / s C$ gene is essential for the growth of $B$. subtilis. In agreement with this essentiality, none of the three different mutations in the six independent mutants results in a frame shift or a deletion. Instead, residue L48 is replaced by a proline in three independently isolated mutants, A61 by a valine in two mutants, and P110 by serine in one mutant. All three residues are located in functionally important regions of the protein. L48 is close to the HXXXXD motif, which is part the active site of the enzyme. Similarly, A61 and P110 are in very close proximity to the active center of the enzyme (S5.6 Fig.; Robertson et al., 2017). The location of all three mutations in the immediate vicinity of the active center as well as the fact that the mutations affect conserved residues suggest that they result in a reduced PIsC activity, and thus in reduced phospholipid biosynthesis.

The requirement of the $\Delta d a c y f k C^{*}$ mutant for an additional mutation reducing lipid biosynthesis to allow growth in the presence of glutamate suggests that the membrane properties are important for controlling cellular glutamate homeostasis. This conclusion is supported by the wellestablished functional interaction between mechanosensitive channels and lipid molecules (Pliotas et al., 2015; Rasmussen et al., 2019). It is tempting to speculate that the combination of altered lipid composition and the mutation in the trans-membrane helix of $\mathrm{YfkC}$ results in a change of channel properties that facilitates the export of glutamate by YfkC.

\section{AimA (YbeC) is a novel major glutamate transporter}

The acquisition of suppressor mutations that provide resistance to glutamate to the $\Delta d a c$ mutant was a rare event that took several days to occur (see above). However, we observed the rapid appearance 
of glutamate-resistant suppressor mutants if the strain already carried a nhaK mutation that facilitates potassium export (see Fig. 5.4). To determine the requirements for glutamate resistance in more detail, we isolated a set of 16 new suppressor mutants (see Fig. 5.1). Since the originally isolated suppressor mutant GP2842 carried a combination of mutations in nhaK and the ybeC gene encoding an amino acid transporter, we sequenced the $y b e C$ allele in all glutamate-resistant suppressor mutants isolated from the $\Delta$ dac nhaK(S187F) (from now on referred to as $n h a K^{*}$ and NhaK* for the gene and protein, respectively). Strikingly, all 16 strains carried mutations in ybeC. Among these mutations are frameshifts in ten strains, an insertion of 2 amino acids after residue 226 in one strain, and amino acid substitutions in the remaining five strains (for details, see Chapter 9.2). The high number of mutants with inactivated $y b e C$ genes strongly suggests that the combination of NhaK* and loss of YbeC allows growth of the $\Delta d a c$ mutant in the presence of glutamate. To test this hypothesis, we deleted the $y b e C$ gene in the $\Delta d a c$ mutant. The resulting strain, GP3054, was unable to grow in the presence of glutamate, indicating that only the combination of the $n h a K^{*}$ and the $y b e C$ mutations conferred resistance to glutamate to the $\Delta$ dac mutant.

Three lines of evidence suggest that $\mathrm{YbeC}$ is the major glutamate transporter of $B$. subtilis: (i) We isolated seven independent mutants affected in $y b e C$ in the initial screen for mutations conferring glutamate resistance to the $\Delta d a c$ strain, (ii) we reproducibly isolated a large number of $y b e c$ mutants based on the $\Delta d a c n h a K^{*}$ mutant, and (iii) $\mathrm{YbeC}$ is an amino acid transporter of the amino acidpolyamine-organocation (APC) superfamily. We have recently demonstrated that $\mathrm{YbeC}$ is the major serine transporter of B. subtilis (Klewing et al., 2020). It is well established that many amino acid transporters can take up multiple different amino acids. To test the possible role of $\mathrm{YbeC}$ in glutamate uptake, we first attempted functional complementation in $E$. coli that is unable to grow with glutamate as the single source of carbon and nitrogen due to the low activity of intrinsic glutamate transporters (Halpern and Lupo, 1965). For this purpose, we transformed E. coli JM109 with plasmid pGP2987 that carries the ybeC gene or with the empty vector pWH844 (Schirmer et al., 1997). The resulting transformants were tested for growth on minimal medium with glucose and ammonium or with glutamate as the only source of carbon and nitrogen (see Fig. 5.5a). Whereas both strains grew well with glucose and ammonium, the strain carrying the empty vector was unable to utilize glutamate. In contrast, the expression of the $y b e C$ gene allowed E. coli to use glutamate as source of carbon and energy. These findings confirm that $\mathrm{YbeC}$ indeed is a glutamate transporter. The protein and the gene were therefore renamed AimA and aimA, respectively (amino acid importer $\underline{A}$ ).

It is very striking that our suppressor screens for glutamate-resistant mutants often identified AimA but never GltT or GltP, the two described glutamate transporters of $B$. subtilis. Therefore, we analyzed the contributions of these proteins to glutamate uptake by comparing the growth of strains 
expressing only one of the three proteins and a strain lacking all of them (see Fig. 5.5b). All tested strains grew well in the presence of ammonium as the nitrogen source, reflecting their ability to synthesize glutamate. The strain GP3071 lacking all three presumptive glutamate transporters was only viable at high glutamate concentrations but not at $1 \mathrm{mM}$ or $5 \mathrm{mM}$. This demonstrates that the bacteria need to transport glutamate if no ammonium is available for its synthesis. Moreover, it demonstrates that efficient glutamate transport is not possible in the absence of the three proteins. GP2818 expressing GltT grew at the three tested glutamate concentrations confirming its role as glutamate transporter. The strain GP2825 that expressed AimA as the only glutamate transporter did not grow at the lowest tested glutamate concentration $(1 \mathrm{mM})$, but this strain was able to grow at $5 \mathrm{mM}$ and $20 \mathrm{mM}$ glutamate suggesting that AimA might be a low-affinity glutamate transporter. In contrast, the strain expressing GItP was only viable at highest glutamate concentration as observed for strain GP3071 lacking all three proteins. This suggests that GItP plays only a very minor, if any role, in glutamate uptake, and that yet another protein can transport glutamate at high concentrations.

A

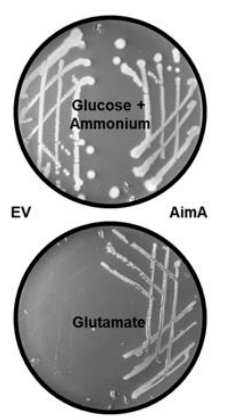

C
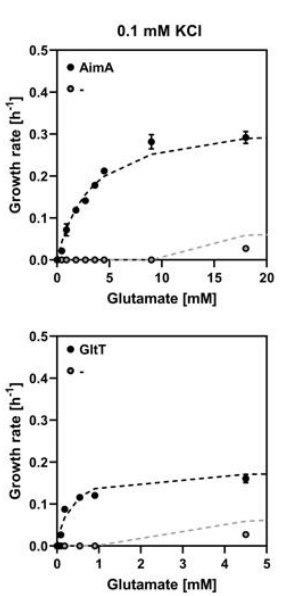

B
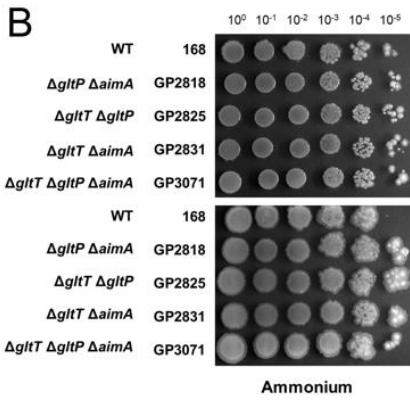

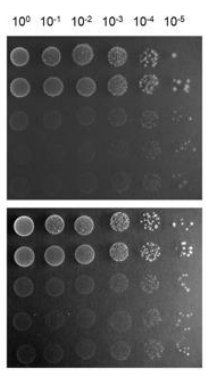

$1 \mathrm{mM}$

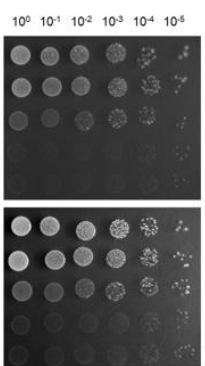

$5 \mathrm{mM}$

$15 \mathrm{~m}$

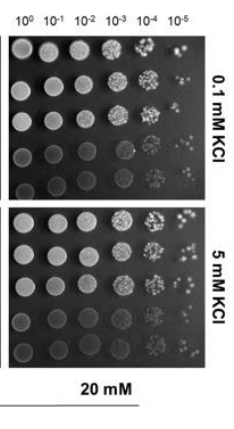

D

\begin{tabular}{|c|c|c|c|c|}
\hline \multirow{2}{*}{} & \multicolumn{2}{|c|}{$\mathbf{0 . 1} \mathbf{m M ~ K C l}$} & \multicolumn{2}{c|}{$\mathbf{5 ~ m M ~ K C I}$} \\
\cline { 2 - 5 } & $\mathbf{K}_{\mathbf{S}}[\mathrm{mM}]$ & $\mathbf{V}_{\max }\left[\mathrm{h}^{-1}\right]$ & $\mathbf{K}_{\mathbf{S}}[\mathrm{mM}]$ & $\mathbf{v}_{\max }\left[\mathrm{h}^{-1}\right]$ \\
\hline AimA & $3.2 \pm 0.5$ & $0.3 \pm 0.03$ & $2.3 \pm 0.33$ & $0.3 \pm 0.005$ \\
\hline GItT & $0.3 \pm 0.02$ & $0.2 \pm 0.01$ & $0.06 \pm 0.03$ & $0.2 \pm 0.003$ \\
\hline- & $12.3 \pm 0$ & $0.1 \pm 0.01$ & $13.8 \pm 1.6$ & $0.3 \pm 0.005$ \\
\hline
\end{tabular}

E

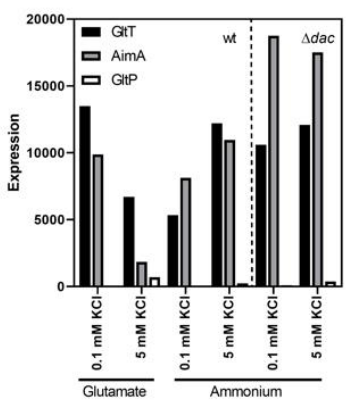

Fig. 5.5. Identification of AimA as a novel low-affinity glutamate transporter. (A) E. coli JM109 was transformed with pGP2987 (AimA) and pWH844 (empty vector) and growth of E. coli JM109 was assessed on MSSM minimal medium with glucose and ammonium or glutamate as carbon and nitrogen source. (B) Growth assay of B. subtilis glutamate transporter mutants. $B$. subtilis strains were cultivated in MSSM minimal medium with $0.1 \mathrm{mM} \mathrm{KCl}$ and ammonium. Cells were harvested, washed, and the $\mathrm{OD}_{600}$ was adjusted to 1.0 . Serial dilutions were dropped 
onto MSSM minimal plates with the indicated potassium concentration and ammonium or glutamate $(1,5$, or 20 $\mathrm{mM}$ ). (C) Determination of glutamate transporter kinetics. The growth rates of $B$. subtilis strains expressing either AimA (upper panel) or GltT (lower panel) as the only glutamate transporter were used to determine the apparent glutamate transporter kinetics of AimA and GltT. A B. subtilis strain lacking all glutamate transporters $(\Delta g / t T$ $\Delta$ aimA $\Delta$ gltP; light grey) served as a control. The strains were grown in MSSM minimal medium with $0.1 \mathrm{mM}$ or $5 \mathrm{mM} \mathrm{KCl}$ and various glutamate concentrations. The growth rate was plotted against the glutamate concentration. (D) Kinetic parameters ( $\mathrm{K}_{s}$ and $\mathrm{V}_{\max }$ values) for AimA and GltT. (E) Expression of gltT, aimA, and $g / t P$. The expression levels of the genes encoding the glutamate transporters GltT, AimA, and GltP were extracted from the transcriptomic analysis.

To study the activities of AimA and GltT in glutamate transport in more detail, we assayed the growth of strains expressing only one of these transporters at different glutamate concentrations (see Fig. 5.5c, 5.5d). Moreover, we performed this experiment in the presence of $0.1 \mathrm{mM}$ and $5 \mathrm{mM} \mathrm{KCl}$ in the medium to see whether potassium has an effect on glutamate uptake as it has been observed with an activating effect of glutamate on potassium uptake by KtrCD (Krüger et al., 2020b). As control, we used strain GP3071 that lacks AimA, GltT, and GltP. The results were used to calculate apparent $\mathrm{K}_{\mathrm{s}}$ values that reflect the affinities of the transporters for glutamate. For GltT, we observed $\mathrm{K}_{\mathrm{s}}$ values of $0.3 \mathrm{mM}$ and $0.06 \mathrm{mM}$ in the presence of 0.1 and $5 \mathrm{mM}$ potassium, respectively. These results are in good agreement with the description of GltT as a high-affinity glutamate transporter (Zaprasis et al., 2015). Moreover, they show that the affinity of GItT for glutamate is five-fold increased in the presence of high potassium concentrations. For AimA, we determined apparent $\mathrm{K}_{\mathrm{s}}$ values of 3.2 and $2.3 \mathrm{mM}$ in the presence of 0.1 and $5 \mathrm{mM}$ potassium, respectively. In good agreement with the drop dilution assay, this suggests that AimA is a low-affinity glutamate transporter, and that the affinity of AimA is not affected by the external potassium concentration. The strain GP3071 lacking all known and suspected glutamate transporters grew only at high glutamate concentrations. This suggests the presence of an additional transporter with very low affinity for glutamate (apparent $\mathrm{K}_{\mathrm{s}}$ values of 12.3 and $13.8 \mathrm{mM}$ in the presence of 0.1 and $5 \mathrm{mM}$ potassium, respectively). Thus, AimA is the major glutamate transporter under standard growth conditions when glutamate is not limiting in the medium.

The role of AimA as the major glutamate transporter is in excellent agreement with the common isolation of glutamate-resistant $\Delta$ dac aimA mutants whereas no gltT mutants could be isolated. Indeed, the $\Delta d a c \Delta g / t T$ mutant was unable to grow in the presence of glutamate, but again a suppressor mutant, GP3093, could be isolated (see Fig. 5.1). Not surprisingly, this strain carries a mutation in aimA (Leu-419 Pro). Moreover, this strain carries frame-shift mutations in $n d h F$ and flik. The paired occurrence of aimA and $n d h F$ suppressor mutations has been observed several times independently (see Table 5.1). 
To get further insights into $g / t P, g / t T$ and $\operatorname{aimA}$, we determined the expression of these genes based on the transcriptome analysis under the relevant conditions (see Fig. 5.5e). The expression of gltP was very low under all tested conditions, which is in agreement with the observation that GltP does not play a significant role in glutamate uptake. The expression of both GltT and AimA was significant. In the presence of glutamate in the medium, the expression was reduced five- and twofold for AimA and GltT, respectively, at the high potassium concentration ( $5 \mathrm{mM})$. In the absence of glutamate, aimA expression did not respond to the supply of potassium whereas the expression of gltT was about two-fold increased at the increased potassium concentration. Thus, if glutamate is limiting, both the expression of $g / t T$ and the affinity of the GltT protein for glutamate are increased by potassium.

\section{Adaptation of a $\Delta$ dac $\Delta$ aimA mutant to growth in the presence of glutamate}

To make the picture of the requirements for a $\Delta d a c$ mutant to tolerate glutamate complete, we also adapted a $\Delta \operatorname{dac} \Delta$ aimA mutant to the presence of glutamate. As described above, this mutant was not viable in the presence of glutamate; however, we could isolate three glutamate-resistant suppressor mutants (see Fig. 5.1). The mutations in these strains were identified by whole genome sequencing. Interestingly, all three suppressor mutants had mutations affecting the essential translation elongation factor G, in two strains (GP3101 and GP3103) the highly conserved Ala-579 was replaced by a Val (see S5.7 Fig.). Ala-579 is located in immediate vicinity of a loop (Loop II) that is required for tRNA translocation in the ribosome (Liu et al., 2014; Peng et al., 2019), suggesting that the mutation interferes with translation. In the third strain (GP3102) we found an A21T substitution. This residue is the immediate neighbour of Asp-20, which binds the $\mathrm{Mg}^{2+}$ ion in the active site of the protein (Maracci et al., 2014). The presence of substitutions in highly conserved and functionally important regions of FusA in all three suppressors suggests that reduced translation efficiency helps the $\Delta$ dac $\Delta a i m A$ mutant to adapt to the presence of glutamate. In addition to the mutations in fusA each strain had a distinct additional mutation. Strain GP3101 had a frameshift mutation in the yqeG gene encoding a putative HAD superfamily phosphatase (Terakawa et al., 2016), whereas the two other strains carried point mutations affecting potassium homeostasis (nhaK and ktrC for GP3102 and GP3103, respectively) again highlighting the toxicity of potassium in the presence of glutamate. Moreover, GP3102 carried amino acid substitutions affecting SigA, the housekeeping sigma factor (F281V) and MrpA (P397L), a subunit of the sodium exporter of B. subtilis, and GP3103 had a point mutation affecting the quasiessential DNA topoisomerase 1 TopA (K13N). The analysis of these strains for growth in the presence of glutamate with 0.1 or $5 \mathrm{mM}$ potassium revealed that all suppressors were viable at both potassium 
concentrations, however, the suppression was most efficient if the yqe $G$ or $k \operatorname{tr} C$ genes were affected in addition to $\operatorname{aimA}$ (see S5.8 Fig.).

\section{DISCUSSION}

Cyclic di-AMP is essential for many bacteria that produce this second messenger; however, the reasons behind this essentiality have not been fully understood. In this study, we used the Gram-positive model organism B. subtilis to gain further insights into the critical functions of c-di-AMP. Based on a variety of distinct suppressor screens to adapt a B. subtilis strain lacking c-di-AMP to either the presence of glutamate or to growth on complex medium, we can conclude that the control of potassium homeostasis is the major bottleneck that limits growth of the mutant under both conditions. This is in good agreement with (i) the large variety of c-di-AMP target proteins and RNA molecules that are involved in the uptake and export of potassium in B. subtilis and other bacteria (Corrigan et al., 2013; Nelson et al., 2013; Gundlach et al., 2017b; Blötz et al., 2017; Devaux et al., 2018b; Quintana et al., 2019; Gundlach et al., 2019; Gibhardt et al., 2019; Stülke and Krüger, 2020), (ii) the fact that the intracellular c-di-AMP levels seem to report the extracellular potassium concentrations (Gundlach et al., 2017b; Zarrella et al., 2018; Pham et al., 2018), and the isolation of suppressor mutants affecting potassium homeostasis in response to altered cellular c-di-AMP levels also in other bacteria (Zarrella et al., 2018; Pham et al., 2018). However, our study also clearly demonstrates that potassium is not the only problem for growth of $B$. subtilis in the absence of c-di-AMP since the presence of mutations that reduce potassium uptake or facilitate its export is not sufficient to overcome the toxicity of glutamate or complex medium. Suppressor studies with L. monocytogenes and S. aureus mutants lacking c-di-AMP revealed that those mutants often had acquired mutations in transporters for osmoprotectants such as glycine betaine indicating that intoxication by compatible solutes is the main reason for essentiality of c-di-AMP in these latter bacteria. The idea of a major role for c-di-AMP in osmoprotection rather than in potassium homeostasis in these pathogenic bacteria is also supported by the observation that potassium has only a minor impact on a L. monocytogenes strain lacking the only diadenylate cyclase (Gibhardt et al., 2019). Thus, while general features of c-di-AMP signaling are conserved in different bacteria, the specific roles and mechanisms may differ even between closely related bacteria.

Potassium and glutamate are the most abundant cation and anion, respectively, in every living cell, and the intracellular concentrations of the two ions need to be balanced (Gundlach et al., 2018). In $B$. subtilis, c-di-AMP seems to play an important role in achieving this balance. The concentrations of the nucleotide respond both to the potassium and the nitrogen source availability, they are high at high potassium concentrations and in the presence of glutamate but not ammonium or glutamine 
(Gundlach et al., 2015b; Gundlach et al., 2017b; Zeden et al., 2018). Our work supports the idea of a close physiological integration of potassium and glutamate homeostasis: Both potassium and glutamate inhibit growth of the $\Delta d a c$ mutant independently of each other. The accumulation of amino acids has also been shown to be a problem for the $\Delta$ dac mutants of L. monocytogenes and S. aureus. As presented in this study, in both organisms amino acid transporters were often inactivated in suppressor mutants that were capable of growing on complex medium despite the lack of c-di-AMP: In L. monocytogenes, several suppressor mutants carried mutations inactivating the OppABCD oligopeptide transporter (Whiteley et al., 2015) whereas the characterization of S. aureus suppressor mutants resulted in the identification of the AlsT glutamine transporter, thus indicating that glutamine rather than glutamate is toxic for the S. aureus $\Delta$ dac mutant (Whiteley et al., 2015; Zeden et al., 2020). Again, these differences between the related organisms support the idea that c-di-AMP has global overarching functions in the different bacteria, but these can be put into practice differently.

It had been established previously that the accumulation of glutamate can be toxic for B. subtilis (Commichau et al., 2008; Gunka and Commichau, 2012). In the case of the $\Delta$ dac mutant, our work has unraveled three ways to overcome glutamate toxicity: (i) Glutamate can be efficiently degraded upon decryptification of the otherwise inactive gudB gene encoding the major constitutive glutamate dehydrogenase of $B$. subtilis. Such a well established gudB $B^{+}$mutation (Belitsky and Sonenshein, 1998) was found in the suppressor mutants GP2841 and GP3473. (ii) The uptake of glutamate can be prevented, and this was indeed observed in many suppressor mutants. Surprisingly, the recently characterized serine transporter AimA ( $\mathrm{YbeC}$ ) was identified as the main glutamate transporter rather than the previsously studied GltT (Zaprasis et al., 2015). Even the $\Delta$ dac mutant lacking gltT was unable to grow in the presence of glutamate unless AimA had been inactivated (see Fig. 5.1). This observation as well as our detailed kinetic analyses revealed that AimA is the major low affinity transporter for glutamate. Moreover, in the absence of c-di-AMP, the aimA gene is the most strongly expressed gene encoding a glutamate transporter (see Fig. 5.5e). It is interesting to note that the expression of aimA responds to the availability of c-di-AMP (see Fig. 5.5b), however, the mechanism responsible for this control has yet to be uncovered. We have previously demonstrated that a potassium channel $(K \operatorname{trCD})$ is directly activated and thus converted from a low- to a high-affinity uptake system in the presence of glutamate (Krüger et al., 2020b). Here, we show that similarly the affinity of the high affinity glutamate transporter GltT is controlled by the presence of potassium: At high potassium concentrations, the affinity of GltT for glutamate is increased fivefold. Thus, the uptake systems for the two most abundant ions in the cell can respond to the presence of the other ion to keep their intracellular concentrations balanced. (iii) In addition to mutations activating glutamate degradation or preventing glutamate uptake, we found several mutants with paired mutations 
affecting lipid biosynthesis and the mechanosensitive channel of small conductivity YfkC. Moreover, the $\Delta d a c y f k C^{*}$ mutant expressing the altered channel protein had to acquire additional mutations affecting either glutamate uptake ( $\operatorname{aim} A)$ or fatty acid biosynthesis $(\operatorname{acc} A)$ to tolerate the otherwise toxic glutamate. We suggest that the altered $\mathrm{YfkC}$ protein in conjunction with altered membrane properties facilitates the export of glutamate. This idea is supported by the observation that $\mathrm{YfkC}$ needs a gain-of-function mutation to bypass glutamate toxicity. Moreover, MscS-like mechanosensitive channels have been shown to export glutamate in Corynebacterium glutamicum (Becker et al., 2013; Wang et al., 2018). In this bacterium, a gain of function mutation affecting this channel results in constitutive glutamate secretion (Nakayama et al., 2012). Finally, specific membrane properties in C. glutamicum such as reduced lipid amounts facilitate glutamate export by the MscS-like channel (see Nakayama et al., 2019 for review). Our results and the parallels in C. glutamicum strongly support the idea that the combination of mutations reducing lipid biosynthesis and that affect the mechanosensitive channel YfkC allow glutamate export in B. subtilis, thus overcoming the toxic effect of this amino acid.

Three lines of evidence suggest that respiration is a major bottleneck for B. subtilis in the absence of c-di-AMP. First, many suppressors isolated in the presence of glutamate had mutations that inactivate the $n d h F$ gene encoding a subunit of a putative NADH dehydrogenase or the downstream $y b c c$ gene of unknown function. It is tempting to speculate that the two proteins form a complex that catalyzes the oxidation of $\mathrm{NADH}_{2}$. Second, when we used complex medium to isolate suppressor mutants, this was initially only possible in the absence of oxygen if respiration activity was low. This is paralleled by the observation that the $S$. aureus $\Delta d a c$ is viable in the absence but not in the presence of oxygen (Zeden et al., 2018). Finally, the lack of c-di-AMP results in an enhanced expression of the $n d h F-y b c C$ operon and reduced intracellular $\mathrm{NADH}_{2}$ levels as indicated by the strong repression of the genes of the Rex regulon in the $\Delta d a c$ mutant. Although it is not yet understood how precisely respiration interferes with the growth of the $\Delta d a c$ mutant, it is tempting to speculate that the proton motive force generated by respiration plays a key role: the major potassium transporter KimA is actually a proton:potassium symporter (Tascón et al., 2020), and potassium uptake by the KtrAB and KtrCD channels is directly driven by the proton motive force (Diskowski et al., 2015). Thus, a reduced respiration lowers the proton motive force and may thus limit the uptake of potassium, the major growth-limiting factor for the $B$. subtilis $\Delta d a c$ mutant.

The adaptation of the $\Delta d a c$ mutant to complex medium did not only confirm the toxicity of potassium, but it did also highlight the particular importance of the two c-di-AMP-binding signal transduction proteins DarA and DarB. The acquisition of suppressor mutations that allow growth under anaerobic conditions on complex medium was only possible when one of these proteins was absent 
(see Fig. 5.1). This indicates that these proteins inhibit growth in their c-di-AMP-free apo form. For DarB it has very recently been shown that the apo protein stimulates the synthesis of ( $p$ )ppGpp by the alarmone synthetase Rel both in B. subtilis and L. monocytogenes (Krüger et al., 2020c; Peterson et al., 2020), and excessive (p)ppGpp synthesis limits growth of both organisms (Whiteley et al., 2015; Krüger et al., 2020c; Peterson et al., 2020). For DarA, no function has been identified so far. It is interesting to note, however, that DarA seems to be even more inhibitory than DarB since the $\Delta$ dac $\Delta$ darB mutants tend to acquire additional mutations affecting DarA when adapting to the presence of oxygen.

Taken together, our study supports the idea that c-di-AMP plays a central role in the physiology of $B$. subtilis. Moreover, by comparing the results obtained with suppressor screens in closely related but distinct bacteria, it becomes clear that the overall themes of c-di-AMP signaling are similar in different bacteria whereas the precise mechanisms are not. While some of the functions of c-di-AMP in B. subtilis such as the control of potassium homeostasis are already well understood, there remain many open questions for future research such as the link between c-di-AMP and lipid biosynthesis and the function of the PII-like signal transduction protein DarA. The work described here provides a solid ground for further investigation.

\section{EXPERIMENTAL PROCEDURES}

\section{Strains, media and growth conditions}

E. coli DH5 $\alpha$ (Sambrook and Russell, 2001) was used for cloning and for the expression of recombinant proteins. E. coli JM109 (Promega) was used for glutamate transporter complementation assays. All B. subtilis strains used in this study are derivatives of the laboratory strain 168 . They are listed in Chapter 9. B. subtilis was grown in Luria-Bertani (LB) or in sporulation (SP) medium (Sambrook and Russell, 2001; Kunst and Rapoport, 1995). For the assay of potassium and glutamate toxicity, B. subtilis was cultivated in MSSM medium (Gundlach et al., 2017b). In this modified SM medium $\mathrm{KH}_{2} \mathrm{PO}_{4}$ was replaced by $\mathrm{NaH}_{2} \mathrm{PO}_{4}$ and $\mathrm{KCl}$ was added as indicated. The media were supplemented with ampicillin $(100 \mu \mathrm{g} / \mathrm{ml})$, kanamycin (10 and $50 \mu \mathrm{g} / \mathrm{ml}$ for B. subtilis and E. coli, respectively), chloramphenicol $(5 \mu \mathrm{g} / \mathrm{ml})$, tetracyclin $(12.5 \mu \mathrm{g} / \mathrm{ml})$, spectinomycin $(150 \mu \mathrm{g} / \mathrm{ml})$ or erythromycin and lincomycin (2 and $25 \mu \mathrm{g} / \mathrm{ml}$, respectively) if required.

\section{Suppressor screen}

For identification of suppressor mutations that rescue the growth of the c-di-AMP lacking strain GP2222, a suppressor screen was set up. The same set-up was performed for the suppressor screens with GP2223, GP2814, GP3054, and GP2259 (see Fig. 5.1). The respective strain was plated on MSSM minimal plates in the presence of ammonium and $0.1 \mathrm{mM} \mathrm{KCl}$. After incubation for 2-3 days, cell 
material was scraped from the plates and washed three times in MSSM basal salts solution. These cells were either plated on MSSM minimal plates (Na-Glutamate, $0.1 \mathrm{mM} \mathrm{KCl}$ ) or used for inoculation of $10 \mathrm{ml}$ MSSM minimal medium (Na-Glutamate, $0.1 \mathrm{mM} \mathrm{KCl}$ ). The bacteria were then incubated at $42^{\circ} \mathrm{C}$ for 3-4 days. As soon as suppressors appeared on the plates, they were picked and re-isolated three times. For liquid cultures, as far as growth could be observed, they were plated. Suppressors were picked and re-isolated again. For the suppressor screen on complex medium, GP2222, GP2420, and GP2779 were plated on LB plates. The plates were incubated at $37^{\circ} \mathrm{C}$ either under standard conditions (aerobic) or in an anaerobic jar to create an anaerobic environment. As soon as suppressors appeared, they were picked and re-isolated three times. For the two step adaptation to complex medium, suppressor mutants previously isolated in the anaerobic screen were plated on LB plates and incubated at $37^{\circ} \mathrm{C}$ under aerobic conditions. As soon as new suppressors appeared, they were picked and reisolated three times. All isolated mutants were analyzed by whole-genome sequencing (see below).

\section{Phenotypic characterization}

Amylase activity was detected on plates containing nutrient broth ( $7.5 \mathrm{~g} /$ liter), $17 \mathrm{~g}$ of Bacto agar per liter (Difco), and $5 \mathrm{~g}$ of hydrolyzed starch per liter (Connaught). The ability to degrade starch was detected by sublimating iodine onto the plates. For quantitative determination of lacZ expression in B. subtilis, cells were grown in MSSM medium with the nitrogen source and potassium concentration as indicated and harvested in the exponential phase. $\beta$-Galactosidase activity was determined with cell extracts obtained by lysozyme treatment as described previously (Sambrook and Russell, 2001). One unit of $\beta$-galactosidase is defined as the amount of enzyme that produces $1 \mathrm{nmol}$ of o-nitrophenol per minute at $28^{\circ} \mathrm{C}$.

To assay growth of $B$. subtilis mutants at different potassium concentrations, the bacteria were inoculated in LB medium and precultured in MSSM medium with $0.1 \mathrm{mM} \mathrm{KCl}$. The cultures were grown until exponential phase, harvested, washed three times in MSSM basal salts solution before the $\mathrm{OD}_{600}$ was adjusted to 1.0. Dilution series were then pipetted onto MSSM plates with ammonium or glutamate and 0.1 or $5 \mathrm{mM}$ potassium.

Complementation in E. coli was assayed by transformation of strain JM109 that is unable to grow with glutamate as the single source of carbon and nitrogen. For this purpose, the strain was transformed with the complementation plasmid pGP2987 (aimA) and the empty vector control pWH844. Growth of the transformants was assessed on M9 minimal medium plates containing either ammonium and glucose or glutamate as carbon and nitrogen source (Halpern and Lupo, 1965). 


\section{DNA manipulation and genome sequencing}

Transformation of E. coli and plasmid DNA extraction were performed using standard procedures (Sambrook and Russell, 2001). All commercially available plasmids, restriction enzymes, T4 DNA ligase and DNA polymerases were used as recommended by the manufacturers. Chromosomal DNA of B. subtilis was isolated as described (Kunst and Rapoport, 1995). B. subtilis was transformed with plasmid and genomic DNA according to the two-step protocol (Kunst and Rapoport, 1995).

To identify the mutations in the suppressor mutants, their genomic DNA was subjected to whole-genome sequencing (Reuß et al., 2017). Briefly, the reads were mapped on the reference genome of B. subtilis 168 (GenBank accession number: NC_000964) (Barbe et al., 2009) using the Geneious software package (Biomatters Ltd., New Zealand) (Kearse et al., 2012). Single nucleotide polymorphisms were considered as significant when the total coverage depth exceeded 25 reads with a variant frequency of $\geq 90 \%$. All identified mutations were verified by PCR amplification and Sanger sequencing.

\section{Construction of mutant strains by allelic replacement}

Gene deletion was achieved by transformation of $B$. subtilis 168 with a PCR product constructed using oligonucleotides to amplify DNA fragments flanking the target genes and an appropriate intervening resistance cassette as described previously (Diethmaier et al., 2011). The mutation $y f k C-R 42 \mathrm{~W}$ was introduced by amplification of regions containing and flanking the mutation from strain GP2837, respectively, and a kanamycin resistance cassette was introduced downstream of $y f k C$ in the noncoding region between $y f k C$ and $y f k D$ (terminator region of both genes present). The integrity of the regions flanking the integrated resistance cassette was verified by sequencing PCR products of about $1,000 \mathrm{bp}$ amplified from chromosomal DNA of the resulting mutant strains.

\section{Plasmids}

Construction of a reporter fusion of the kimA promoter region with the promoterless lacZ gene was done using the plasmid pAC6 (Stülke et al., 1997). The promoter fragment was cloned between the EcoRI and BamHI sites of pAC6. The resulting plasmid was pGP2912. The plasmid was linearized with Scal and transformed into B. subtilis 168 for chromosomal integration into the amyE locus. For E. coli complementation assays, the aimA gene was amplified using the primer pair LK167 (5' AAAGGATCCATGAATCAATTGCATCGAAGAATGGGAACGTTTT)/ AK324

TTTGTCGACTTATTCTTTTCCGGCAGCAGCTTCTG) and cloned between the BamHI and Sall sites of the expression vector pWH844 (Schirmer et al., 1997). The resulting plasmid was pGP2987. 


\section{Determination of apparent glutamate transporter kinetics}

The growth characteristics of $B$. subtilis glutamate transporter mutants were used to determine the single glutamate transporter kinetics. The mutants were either positive for gltT( $\Delta g / t P:: c a t \Delta a i m A:: k a n)$ or $\operatorname{aimA}(\Delta g / t T:: k a n \Delta g / t P:: c a t)$ or lacked both ( $\Delta g / t T:: k a n \Delta a i m A:: p h l e o \Delta g / t P:: c a t)$. The nitrogen source was used as the limiting factor, so that transport of glutamate was the essential factor for growth. The bacteria were inoculated in LB medium and precultured in MSSM medium (ammonium) with $0.1 / 5 \mathrm{mM} \mathrm{KCl}$. The cultures were grown until exponential phase and the cells were washed three times in MSSM basal salts solution. Afterwards, the cells were adjusted to $\mathrm{OD}_{600} 1.0$ and used to inoculate a 96 well plate (Microtest Plate 96 Well, F, Sarstedt) containing the MSSM medium with the respective glutamate and potassium concentrations. The growth was tracked in an Epoch 2 Microplate Spectrophotometer (BioTek Instruments) at $37^{\circ} \mathrm{C}$ with linear shaking at $237 \mathrm{cpm}(4 \mathrm{~mm})$ for $20 \mathrm{~h}$, and optical density at $600 \mathrm{~nm}$ was measured in $10 \mathrm{~min}$ intervals. The exponential growth phase was used to determine the growth rate $\mu\left[h^{-1}\right]$. $\mu$ was calculated from $t_{1}$ (early exponential phase) and $t_{2}$ (late exponential phase) and the corresponding $\mathrm{OD}_{1}$ and $\mathrm{OD}_{2}$ values. The resulting growth rates were then plotted against the glutamate concentrations. This allowed fitting to the Monod equation and calculation of $\mathrm{V}_{\max }\left[\mathrm{h}^{-1}\right]$ and the apparent $\mathrm{K}_{\mathrm{s}}$ [mM glutamate] using the solver tool of Excel 2012 (Microsoft). Experiments were repeated with three biological replicates.

\section{Transcriptome analysis}

Wild type and the c-di-AMP-deficient strains GP2222 $\Delta$ dac and GP2223 $\Delta$ dac nhaK-S187F were grown in MSSM medium with ammonium or glutamate and low $(0.1 \mathrm{mM})$ or high $(5 \mathrm{mM})$ potassium concentrations. The strains were harvested by centrifugation $\left(10.397 \times \mathrm{g}, 1 \mathrm{~min}, 4^{\circ} \mathrm{C}\right)$ at mid exponential phase $\left(\mathrm{OD}_{600}\right.$ of 0.4-0.6). A total of two independent biological replicates were included. The pellets were frozen immediately in liquid nitrogen and stored at $-80^{\circ} \mathrm{C}$. Isolation of the total RNA was done as described previously (Eymann et al., 2002; Nicolas et al., 2012), and the quality of the RNA preparations was assessed by means of an Agilent 2100 Bioanalyzer according to the manufacturer's instructions. $5 \mu \mathrm{g}$ of total RNA were used for CDNA synthesis. The synthesis and fluorescence labeling of cDNA followed a strand-specific method using the FairPlay III Microarray Labeling Kit (Agilent Technologies, Santa Clara, CA, USA) and actinomycin D (Calbiochem) (Mäder and Nicolas, 2012). 200 ng of Cy3-labeled cDNA were hybridized to the microarray following Agilent's hybridization, washing and scanning protocol (One-Color Microarray-based Gene Expression Analysis, version 5.5). Data were extracted and processed using the Feature Extraction software (version 11.5.1.1). An aggregated expression value was computed for each annotated coding sequence and previously identified RNA feature (Nicolas et al., 2012). Gene-level intensities were scaled based on the intensity 
values of ten different in vitro-synthesized transcripts contained in the One Color RNA Spike-In kit (Agilent Technologies) in order to account for technical variation associated with sample processing. Genes with at least 2.5-fold difference in expression levels of the wild type at the different conditions or between wild type and GP2222 mutant were considered significantly affected.

To analyze the regulatory network, we compared the expression levels of the wild type strain and the GP2222 mutant at ammonium $0.1 \mathrm{mM} \mathrm{KCl}$. The genes were clustered in operons according to their genetic localization, as described in SubtiWiki (Zhu and Stülke, 2018). Additionally, gathered operons were grouped by their corresponding documented regulator/s and metabolism. The visual representation of the regulatory network was generated with Cytoscape and GIMP softwares (Shannon et al., 2003; GIMP, 2019).

\section{SUPPLEMENTARY MATERIAL}

https://www.biorxiv.org/content/10.1101/2020.09.03.280651v2

S5.1 Supporting Information. Global gene expression in the B. subtilis wild type strain in response to the potassium concentration and the nitrogen source.

S5.1 Table. Transcriptomic data for the genes that are most strongly affected by the absence of c-di-AMP (Ammonium 0.1 mM KCl).

S5.2 Table. Transcriptomic data of genes involved in c-di-AMP, potassium, and glutamate homeostasis.

Fig. S5.1. Acquisition of beneficial mutations allows growth of a c-di-AMP deficient strain in the presence of glutamate.

Fig. S5.2. Potassium and glutamate influenced promoters.

Fig. S5.3. Two-step adaptation to complex medium.

Fig. S5.4. Mutations in the mechanosensitive channel YfkC.

Fig. S5.5. Mutations in the acetyl-CoA carboxylase subunits.

Fig. S5.6. Mutations in the acyl-ACP:1-acylglycerolphosphate acyltransferase.

Fig. S5.7. Mutations in the elongation factor G (FusA).

Fig. S5.8. Effect of the deletion of the glutamate transporters in a c-di-AMP deficient strain.

\section{DATA AVAILABILITY STATEMENT}

The transcriptomic data reported in this study have been submitted to the NCBI Gene Expression Omnibus (GEO; http://www.ncbi.nlm.nih.gov/geo/) under accession number GSE156738. 


\section{ACKNOWLEDGEMENTS}

We are grateful to Anika Klewing, Marina Rodnina, Fabian Commichau, and Frank Peske for helpful discussion. We like to thank Felix Mehne and Martin Weiss for the help with strain constructions and the initial characterization of a suppressor mutant, respectively. 


\section{Chapter 6 - Nucleotide second messenger crosstalk}

The results described in this chapter were originally published as a preprint on bioRxiv (doi: https://doi.org/10.1101/2020.08.27.268672):

\section{A rendezvous of two second messengers: The c-di-AMP receptor protein DarB controls (p)ppGpp synthesis in Bacillus subtilis}

Larissa Krüger ${ }^{1}$, Christina Herzberg ${ }^{1}$, Dennis Wicke ${ }^{1}$, Heike Bähre ${ }^{2}$, Jana L. Heidemann ${ }^{3}$, Achim Dickmanns $^{3}$, Kerstin Schmitt ${ }^{4}$, Ralf Ficner ${ }^{3}$ and Jörg Stülke ${ }^{1}$

${ }^{1}$ Department of General Microbiology, Institute for Microbiology \& Genetics, GZMB, Georg-AugustUniversity Göttingen, 37077 Göttingen, Germany

${ }^{2}$ Research Core Unit Metabolomics, Hannover Medical School, 30625 Hannover, Germany

${ }^{3}$ Department of Molecular Structural Biology, Institute for Microbiology \& Genetics, GZMB, GeorgAugust-University Göttingen, 37077 Göttingen, Germany

${ }^{4}$ Department of Molecular Microbiology and Genetics, Service Unit LCMS Protein Analytics, Institute for Microbiology \& Genetics, GZMB, Georg-August-University Göttingen, 37077 Göttingen, Germany

\section{AUTHOR CONTRIBUTIONS}

LK, RF and JS conceived the study and designed the experiments. LK and $\mathrm{CH}$ performed the bacterial two-hybrid experiments and the protein pulldown assays. LK, CH and DW purified the proteins and studied the affinity of DarB for Rel and cyclic di-AMP with ITC experiments. LK, JLH, and AD performed SEC-MALS experiments. KS analyzed the protein fractions from the pulldown experiments with mass spectrometry. LK and $\mathrm{CH}$ conducted the enzymatic assays and extracted the nucleotides. $\mathrm{HB}$ determined the nucleotide levels. LK, KS, and HB analysed the MS data. LK and JS wrote the original draft of the manuscript. JLH, AD, KS, and RF reviewed and edited the manuscript. All authors approved the manuscript. RF and JS acquired funding. AD, RF, and JS provided supervision. 


\section{ABSTRACT}

Many bacteria use cyclic di-AMP as a second messenger to control potassium and osmotic homeostasis. In Bacillus subtilis, several c-di-AMP binding proteins and RNA molecules have been identified. Most of these targets play a role in controlling potassium uptake and export. In addition, c-di-AMP binds to two conserved target proteins of unknown function, DarA and DarB, that exclusively consist of the c-di-AMP binding domain. Most likely these proteins transduce their signal by regulatory interactions with other proteins. Here, we have investigated the function of the c-di-AMP-binding protein DarB in B. subtilis, a protein consisting of two CBS (cystathionine-beta synthase) domains. We have used an unbiased search for DarB interaction partners and identified the (p)ppGpp synthetase/hydrolase Rel as a major interaction partner of DarB. (p)ppGpp is another second messenger that is formed upon amino acid starvation and under other stress conditions to stop translation and active metabolism. The interaction between DarB and Rel only takes place if the bacteria grow at very low potassium concentrations and intracellular levels of c-di-AMP are low. Indeed, c-di-AMP inhibits the binding of DarB to Rel. The interaction results in the Rel-dependent accumulation of pppGpp. Our results link potassium and c-di-AMP signaling to the stringent response and thus to the global control of cellular physiology.

\section{INTRODUCTION}

All living cells contain high concentrations of potassium ions (Gundlach et al., 2018; Danchin and Nikel, 2019). This ion is required for the activity of many enzymes and protein complexes, among them the ribosome, for buffering the negative charge of the DNA and for osmoadaptation (Epstein, 2003; Gundlach et al., 2018). On the other hand, potassium may become toxic if the intracellular concentration becomes too high (Gundlach et al., 2018). Therefore, potassium homoestasis has to be carefully controlled. In many bacteria, a second messenger - cyclic di-AMP (c-di-AMP) - is involved in the control of potassium homeostasis (Commichau et al., 2018a; Gundlach et al., 2019). The nucleotide is synthesized at high potassium concentrations whereas low c-di-AMP levels indicate a potassium limitation (Gundlach et al., 2017b). The control of the homeostasis of potassium and other osmolytes is the reason that c-di-AMP is essential for many of the bacteria that produce this signaling nucleotide (Stülke and Krüger, 2020). c-di-AMP acts by binding to a variety of targets to control their activity (He et al., 2020; Stülke and Krüger, 2020). Among the targets of c-di-AMP are several proteins, like the potassium importers and exporters and a two-component sensor kinase as well as a riboswitch that are involved in the control of potassium homeostasis. Of all known second messenger nucleotides c-di-AMP is unique in binding and controlling both a protein and the mRNA molecule that encodes it. This is the case for the Bacillus subtilis KtrA and KimA potassium transporters that are both bound and 
thus inhibited by c-di-AMP. In addition, the corresponding mRNAs each carry a c-di-AMP responsive riboswitch, and binding of c-di-AMP prevents the expression of the transporters (Nelson et al., 2013; Gundlach et al., 2017b; Gundlach et al., 2019).

In $B$. subtilis and the related pathogen Listeria monocytogenes, the analysis of c-di-AMPbinding proteins identified two potential signal transduction proteins of unknown function, DarA and DarB (Sureka et al., 2014; Gundlach et al., 2015a; Gundlach et al., 2019). DarA belongs to the large family of PII-like signaling proteins that control a variety of processes mainly in nitrogen metabolism (Forchhammer and Lüddecke, 2016). The DarB protein consists of a tandem of two CBS (cystathioninebeta synthase) domains, an arrangement called Bateman domain (Bateman, 1997). CBS domains bind AMP, ATP, or other adenosine-derived molecules. CBS domains are present in a variety of proteins, including osmolyte and metal ion transporters, enzymes, and transcription regulators. Recently, CBS domain-containing osmolyte and magnesium transporters were found to bind c-di-AMP. In the case of the osmolyte transporters, the proteins are inactivated upon c-di-AMP binding (Schuster et al., 2016; Huynh et al., 2016; Gundlach et al., 2019). Interestingly, in contrast to most other c-di-AMP-binding proteins, DarA and DarB do not contain any other domain that might be controlled by the binding of the second messenger. It is therefore likely that these proteins interact with other proteins in a c-di-AMP-dependent manner to control their activity.

In this study, we performed an unbiased search for potential interaction partners of the DarB protein. This search identified the Rel protein that synthesizes and degrades the alarmone nucleotide (p)ppGpp. The accumulation of this signaling nucleotide results in a global switch off of cellular activities in bacteria, including DNA replication, nucleotide biosynthesis, transcription of household genes, and translation (Hauryliuk et al., 2015; Steinchen and Bange, 2016). Thus, the integration of c-di-AMP and (p)ppGpp signaling allows a global cellular response to the availability of potassium.

\section{RESULTS}

\section{Identification of Rel as an interaction partner of DarB}

We assumed that DarB might act by interaction with other proteins. A L. monocytogenes strain lacking c-di-AMP is unable to grow on complex media, but suppressor mutants with the inactivated homolog of DarB (CbpB) were able to grow on complex medium (Whiteley et al., 2015). This observation suggests that the apo-form of DarB exerts some harmful interactions. In both B. subtilis and L. monocytogenes, DarB is encoded in a conserved operon with the transcription factor $\mathrm{CcpC}$, the regulator of the citric acid cycle (Jourlin-Castelli et al., 2000; Blencke et al., 2006). We hypothesized that DarB might control the activity of CcpC. However, attempts to detect an interaction between the two proteins failed suggesting that DarB exerts a different function (Supplementary Fig. 6.1). 


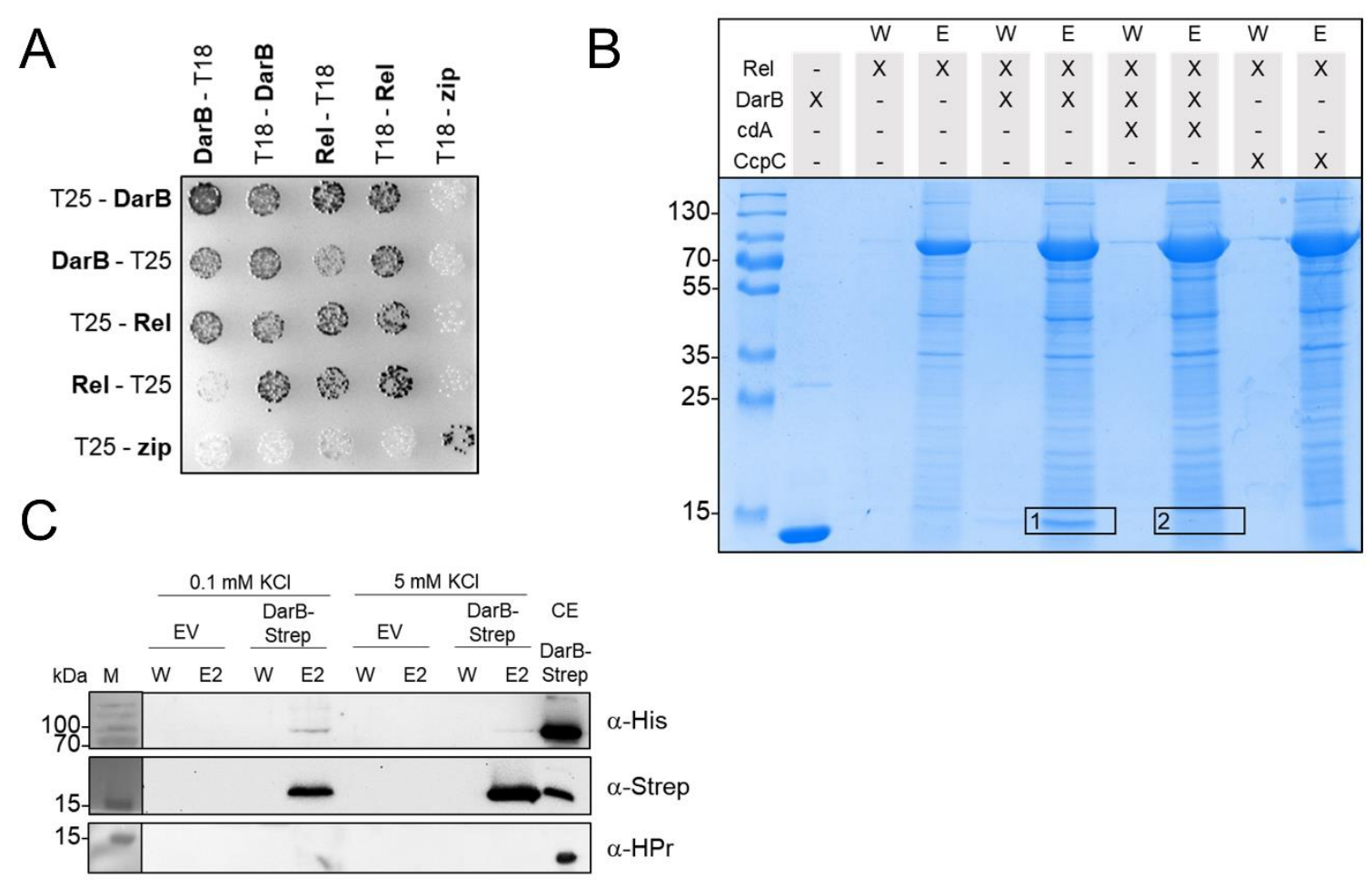

Fig. 6.1. DarB interacts with Rel in vitro and in vivo. (A) Bacterial two-hybrid (BACTH) experiment testing for the interaction of DarB with Rel. N- and C-terminal fusions of DarB and Rel to the T18 or T25 domains of the adenylate cyclase (CyaA) were created and the proteins were tested for interaction in E. coli BTH101. Dark colonies indicate an interaction that results in adenylate cyclase activity and subsequent expression of the reporter $\beta$-galactosidase. (B) In vitro Strep-Rel pulldown experiment. Strep-Rel was immobilized onto a StrepTactin column and incubated with DarB, DarB preincubated with c-di-AMP, or the control protein CcpC. The eluates (E) and wash (W) fractions were analyzed by SDS-PAGE and the presence of DarB in the elution fractions was further verified by MS analysis (excised gel bands are numbered with 1 and 2). (C) In vivo interaction experiment of DarBStrep with Rel-His. B. subtilis expressing Rel-His6 was transformed with plasmid-borne DarB-Strep and grown in minimal medium containing low $(0.1 \mathrm{mM})$ or high $(5 \mathrm{mM})$ potassium concentration. DarB together with its potential binding partners was purified with a StrepTactin column and the elution and wash fractions were analyzed by Western blot analysis. DarB and Rel were detected by using antibodies against the Strep-tag and the His-tag, respectively. HPr served as a negative control. Abbreviations: EV, empty vector; CE, cell extract; cdA, c-d-AMP.

To get a first unbiased glimpse on the function of DarB, we identified potential interaction partners by passing a $B$. subtilis crude extract over a DarB-saturated column. The proteins were then eluted from the column, and the co-purified proteins were identified by mass spectrometry. In agreement with previous results, $\mathrm{C} c \mathrm{C}$ was not identified in the fraction that co-elutes with DarB. In contrast, the analysis identified the GTP pyrophosphokinase Rel as a top scoring protein (Supplementary Table 6.1). This protein was not detected in the negative control and was therefore considered as a putative interaction partner of DarB. Rel catalyzes the production of the alarmones 
ppGpp and pppGpp by transferring pyrophosphate derived from ATP to GDP and GTP, respectively, under conditions of amino acid starvation. Moreover, Rel degrades both alarmones if amino acids become available (Takada et al., 2020).

In order to gain further evidence for the interaction between DarB and Rel, we used the bacterial two-hybrid system in which an adenylate cyclase is reconstituted if cloned proteins interact with each other resulting in $\beta$-galactosidase activity. As shown in Fig. 6.1a, both DarB and Rel exhibited self-interaction, in agreement with structural analysis of these proteins (Avarbock et al., 2005). In addition, co-expression of DarB and Rel resulted in the reconstitution of a functional adenylate cyclase, thus confirming the interaction of the two proteins. None of the two proteins showed an interaction with the Zip protein, which was used as the negative control. Thus, the interaction between DarB and Rel is specific.

Furthermore, we performed size exclusion chromatography-multiangle light scattering (SECMALS) experiments with DarB and the purified Rel protein (see Supplementary Fig. 6.2) to get in vitro confirmation for the interaction. As shown in Supplementary Fig. 6.3b, the two protein co-elute in vitro. In contrast, no co-elution was detectable when DarB was saturated with c-di-AMP (Supplementary Fig. 6.3c). This observation suggests that only apo-DarB is capable of interacting with Rel. It is in agreement with the initial pull-down experiment and the bacterial two-hybrid analysis that both revealed an interaction between the two proteins in the absence of c-di-AMP.

To obtain additional evidence for the specificity of the interaction, we mutated the DarB protein in a way to prevent the interaction with Rel. An inspection of the DarB structure (PDB code 1YAV) as well as of the structure of the DarB-c-di-AMP complex (Heidemann and Ficner, unpublished results) suggested that surface residues close to the c-di-AMP binding site might interfere with Rel binding. We exchanged Ala-25 and Arg-132 to Gly and Met, respectively, in single mutants, and combined the two mutations. The resulting DarB ${ }^{A 25 G, R 132 M}$ was tested for c-di-AMP and Rel binding. Isothermal titration calorimetry (ITC) experiments indicated that the mutated protein binds c-di-AMP (Supplementary Fig. 6.4a) demonstrating that the protein folds correctly. However, a SEC-MALS analysis showed that the mutant protein binds much weaker to Rel as compared to the wild type protein. Moreover, this residual interaction is not affected by c-di-AMP (Supplementary Fig. 6.3d, e).

Taken together, these data indicate that DarB specifically binds to Rel, and that this interaction is inhibited by c-di-AMP.

\section{Biochemical and pyhsiological regulation of the interaction}

To further investigate the role of c-di-AMP in the interaction between DarB and Rel, we assayed the binding of purified DarB to immobilized Strep-tagged Rel in the absence or presence of c-di-AMP. While 
DarB was co-eluted with Rel in the absence of c-di-AMP, no DarB was retained on the column when c-di-AMP was present (see Fig. 6.1b). LC-MS analysis of the gel segments corresponding to the size of DarB confirmed this result (Fig. 6.1b, segments 1 and 2). No interaction between Rel and the negative control CcpC was detected (Fig. 6.1b, see also Supplementary Fig. 6.5, Supplementary Table 6.2). Similarly, the interaction was abolished if the DarB protein had mutations affecting A25 alone or in combination with the R132M substitution (Supplementary Fig. 6.6). These results support the specific interaction between Rel and DarB, and they confirm that Rel interacts with the apo-form of DarB but not with the DarB/ c-di-AMP complex.

c-di-AMP is a second messenger that functions in potassium homeostasis, and the intracellular levels of the nucleotide correlate with the potassium concentration (Gundlach et al., 2018). We tested therefore, how the external potassium supply would affect the interaction between DarB and Rel in vivo. For this purpose, we used a strain that expressed His-tagged Rel from the chromosome and Strep-tagged DarB from a plasmid. This strain was cultivated in minimal medium at low $(0.1 \mathrm{mM})$ and high ( $5 \mathrm{mM}$ ) potassium concentrations, and the protein extract was passed over a StrepTactin column to isolate Strep-DarB in complex with its potential interaction partners (Fig. 6.1C). The presence of the Rel protein in the elution fractions was analyzed by a Western blot using antibodies specific for the His-tag. While His-Rel was co-eluted with DarB at the low potassium concentration, no interaction was detected when the bacteria had been cultivated at the high potassium concentration. Again, the presence and absence of Rel in eluates from cultures grown at 0.1 or $5 \mathrm{mM}$ potassium, respectively, was verified by mass spectrometry (Supplementary Table 6.3). No Rel was detectable in the eluate of the culture grown at the high potassium concentration. Since the intracellular c-di-AMP concentration is low at an external potassium concentration of $0.1 \mathrm{mM}$ (Gundlach et al., 2017b), we conclude that the interaction between DarB and Rel occurs at low potassium concentrations when c-di-AMP is not bound to DarB. This conclusion is in excellent agreement with the observed inhibition of the interaction by c-di-AMP (see Fig. 6.1b).

\section{DarB does not interact with other small alarmone synthetases}

In addition to Rel, B. subtilis encodes two additional (p)ppGpp synthesizing enzymes, the small alarmone synthetases SasA and SasB (Nanamiya et al., 2008). In contrast to Rel, which is a multidomain protein (see below), the latter proteins consist of a stand-alone synthetase domain. To test whether these proteins are also capable of interacting with DarB, we made use of the two-hybrid system as described above for Rel. In agreement with the known formation of homotetramer (Brown et al., 2016), we observed self-interactions for both SasA and SasB. This also indicates that the fusion proteins have folded correctly. Again, we confirmed the interaction between Rel and DarB. However, no 
interaction of SasA and SasB with DarB could be detected (see Supplementary Fig. 6.7). The absence of an interaction between DarB and the small alarmone synthetases is supported by the fact that the proteins did not co-elute with Strep-DarB in the in vivo experiments described above. Thus, the interaction of DarB is most likely specific for Rel.

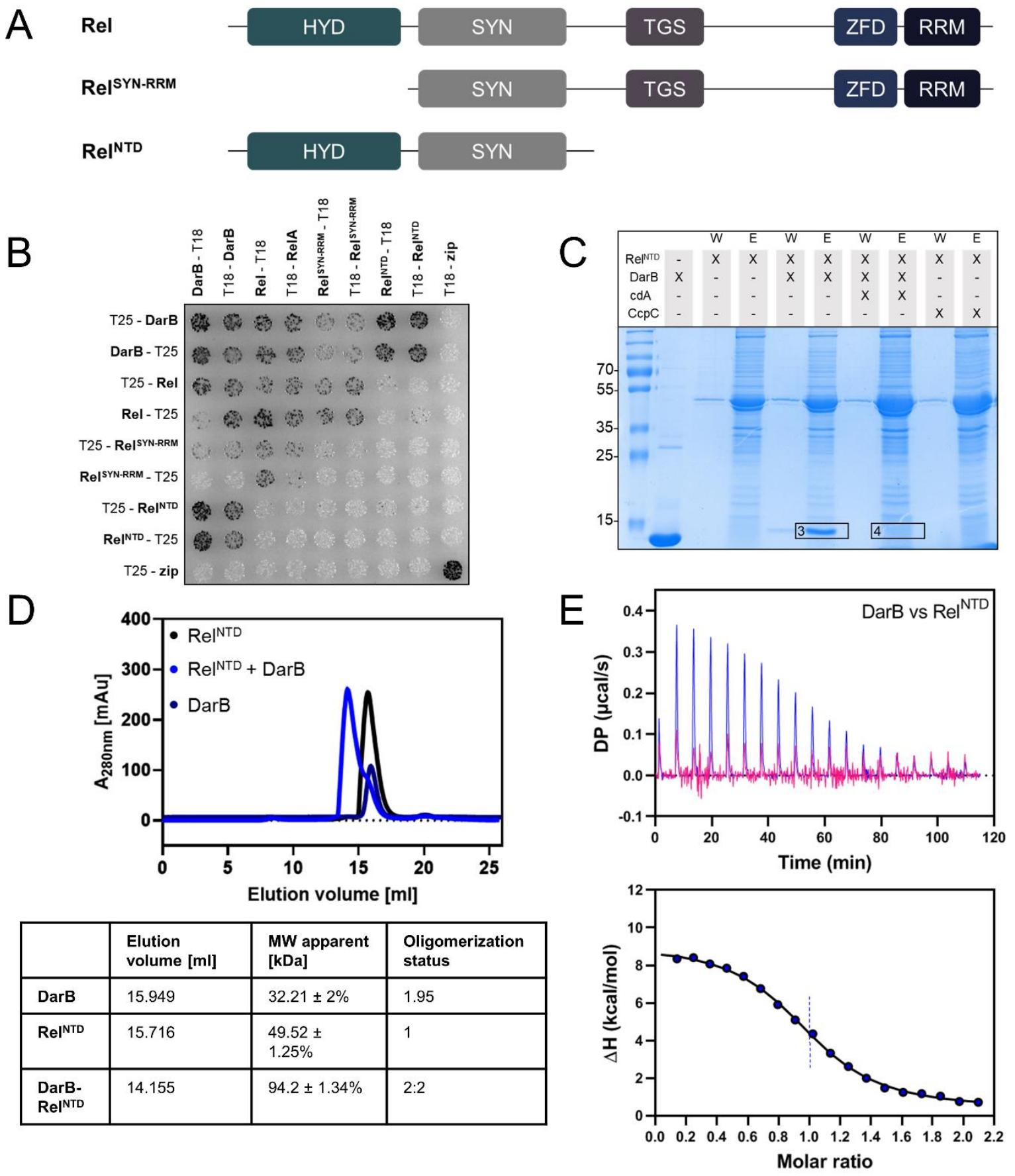

Fig. 6.2. DarB binds the N-terminal domain (NTD) of Rel. (A) The domain organization of Rel and the truncated Rel variants used in this study. Abbreviations: HYD, hydrolase domain; SYN, synthetase domain; TGS, TGS domain (for: ThrRS, GTPase and SpoT); ZFD, a zinc finger domain; RRM domain (for Ribosomal Recognition Motif). (B) Bacterial two-hybrid (BACTH) assay to test for the interaction between DarB and the full-length and truncated 
Rel-variants. $\mathrm{N}$ - and C-terminal fusions of DarB and the Rel variants to the T18 or T25 domain of the adenylate cyclase (CyaA) were created and the proteins were tested for interaction in E. coli BTH101. Dark colonies indicate an interaction that results in adenylate cyclase activity and subsequent expression of the reporter $\beta$-galactosidase. (C) In vitro pulldown experiment with the NTD of Rel. Strep-Rel ${ }^{\text {NTD }}$ was immobilized onto a StrepTactin column and incubated with DarB, DarB preincubated with c-di-AMP, or the control protein CcpC. The eluate and wash fractions were analyzed by SDS-PAGE and the presence of DarB in the elution fractions was further verified by MS analysis (excised gel bands are numbered with 3 and 4). (D) The DarB-Rel ${ }^{\text {NTD }}$ complex was analyzed by size exclusion chromatography and multi-angle light scattering (SEC-MALS). Rel ${ }^{\text {NTD }}$ and DarB were used in equimolar concentrations. Dark blue line, DarB; black line, Rel ${ }^{\mathrm{NTD}}$; blue line, mixture of DarB and Rel. The calculated molar masses determined by MALS are listed below the chromatogram. (E) The molar ratio of the DarB-Rel ${ }^{\text {NTD }}$-complex was assessed by Isothermal titration calorimetry (ITC). The cell and the syringe contained $10 \mu \mathrm{M}$ Rel $^{\text {NTD }}$ and $100 \mu \mathrm{M}$ DarB (blue) or $100 \mu \mathrm{M}$ c-di-AMP-bound DarB (DarB ${ }^{\text {cdA }}$ ) (pink), respectively. Abbreviation: cdA, c-di-AMP.

\section{DarB interacts with the $\mathbf{N}$-terminal portion of Rel}

The Rel protein is a multidomain protein that consists of a $\mathrm{N}$-teminal hydrolase (HYD) domain, the synthetase (SYN) domain, the TGS domain (for: ThrRS, GTPase and SpoT), a zinc finger domain (ZFD), and the C-terminal RNA recognition motif (RRM) domain (see Fig. 6.2a; Brown et al., 2016). While the HYD and SYN domains are required for the degradation and synthesis of (p)ppGpp, respectively, the Cterminal domains are involved in the interaction with the ribosome and the control of the enzymatically active domains (Brown et al., 2016). To test the contribution of the $\mathrm{N}$ - and C-terminal regions of Rel to the interaction with DarB, we analysed the protein-protein interactions using the bacterial two-hybrid system (see Fig. 6.2b). The Rel fragment consisting of the SYN domain and the Cterminal regulatory domains showed a very faint interaction with DarB. In contrast, a very strong interaction was observed for the $\mathrm{N}$-terminal fragment consisting of the HYD and the SYN domains $\left(\operatorname{Re}^{\mathrm{NTD}}\right)$. Thus, in contrast to the interaction of Rel with the ribosome which is mediated by the Cterminal RRM domain, DarB seems to bind to the N-terminal part of Rel.

To confirm the binding of the N-terminal region of Rel to DarB, we assayed binding of DarB to the immobilized truncated Rel ${ }^{\text {NTD }}$ protein that lacked the C-terminal part. As observed for the fulllength protein, this HYD-SYN fragment of Rel bound to DarB, and this interaction was prevented by the addition of c-di-AMP (Fig. 6.2c, Supplementary Table 6.2).

In order to confirm the complex formation of Rel ${ }^{\mathrm{NTD}}$ and DarB in vitro, a SEC-MALS experiment was performed. The separated elution profiles of the two proteins correspond to a monomer for Rel ${ }^{\mathrm{NTD}}$ and a dimer for DarB. A dimer formation by DarB is in agreement with the results from the two-hybrid analysis (Fig. 6.1a) and the available crystal structure of the apo-protein (PDB 1YAV). By contrast, Rel ${ }^{\text {NTD }}$ was unable to exhibit self-interactions in the two-hybrid screen (see Fig. 6.2b). Co-elution of Rel ${ }^{\mathrm{NTD}}$ and DarB resulted in an earlier eluting peak, indicating the formation of a complex of $94.2 \mathrm{kDa}$ consisting 
of DarB and Rel ${ }^{\text {NTD }}$ (see Fig. $6.2 d$, Supplementary Fig. 6.8). The subsequent SDS page analysis of the elution fractions confirmed that both proteins co-eluted from the column (see Supplementary Fig. 6.8). To determine the kinetic parameters of the interaction, we performed isothermal titration calorimetry (ITC) experiments (Fig. 6.2e). Titration of DarB against Rel ${ }^{\text {NTD }}$ revealed an equimolar stoichiometry of the two proteins in the complex. Moreover, we determined the affinities of DarB for c-di-AMP and Rel. While the $\mathrm{K}_{\mathrm{D}}$ for the binding of c-di-AMP was about $45 \mathrm{nM}$ (Supplementary Fig. 6.4a, b), we observed a $K_{D}$ of $650 \mathrm{nM}$ for the interaction of DarB and Rel (see Fig. 6.2e). This about 15-fold higher affinity of DarB for c-di-AMP is crucial for the c-di-AMP-mediated regulation of the DarB-Rel interaction.
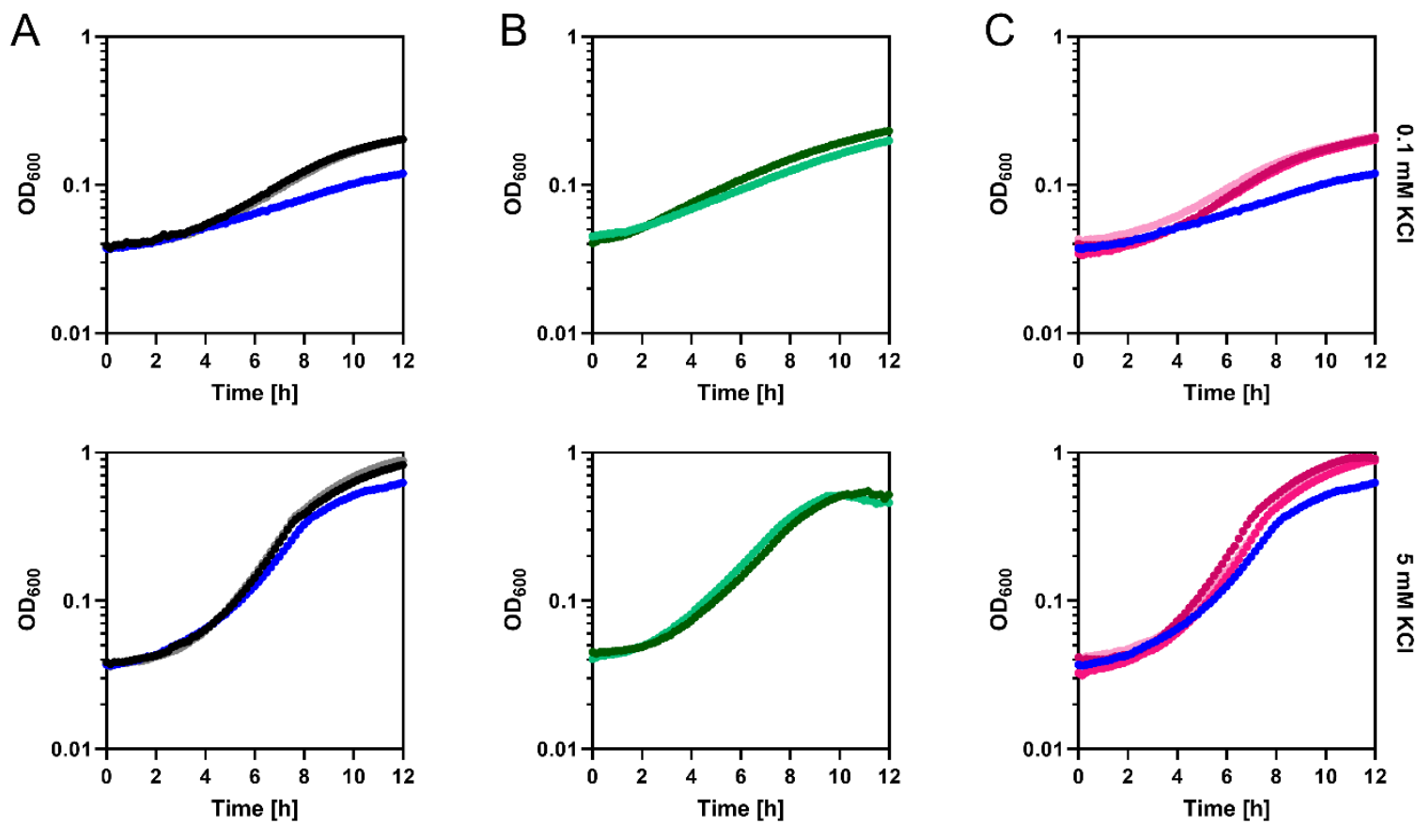

- WtEV

- $\Delta r e l E V$

- Wt darB

- Wt darB

- $\Delta$ rel darB

- Wt darB ${ }^{\mathrm{A} 25 \mathrm{G}, \mathrm{R} 132 \mathrm{M}+}$

- Wt darB ${ }^{\mathrm{A} 25 \mathrm{G}+}$

- Wt darB ${ }^{\mathrm{R} 132 \mathrm{M}+}$

Fig. 6.3. Overexpression of DarB is toxic. Growth experiments of (A) wild type B. subtilis (black), GP3407 ( $\triangle$ darB; grey), wild type + pGP3306 (darB ${ }^{+}$, blue), (B) GP3419 ( $\Delta r e l$, bright green) and GP3419 ( $\Delta r e l$, darB ${ }^{+}$, dark green), and (c) wild type + pGP3437/3441/3601 (darB ${ }^{\mathrm{A} 25 \mathrm{G}}$, $\operatorname{darB}^{\mathrm{R} 132 \mathrm{M}}, \operatorname{darB}^{\mathrm{A25G}, \mathrm{R} 132 \mathrm{M}}$, red) in MSSM minimal medium with $0.1 \mathrm{mM} \mathrm{KCl}$ (upper panel) or $5 \mathrm{mM} \mathrm{KCl}$ (lower panel). Growth was monitored in an Epoch 2 Microplate Spectrophotometer (BioTek Instruments) at $37^{\circ} \mathrm{C}$ with linear shaking at $237 \mathrm{cpm}(4 \mathrm{~mm})$ for $12 \mathrm{~h}$.

\section{Genetic support for the DarB-Rel interaction}

So far, no function other than binding to c-di-AMP and to Rel has been identified for DarB. To get better insights into the physiological role(s) of DarB, we constructed strains that either lacked DarB ( $\Delta$ darB, GP3409) or that overexpressed the protein $\left(\right.$ darB $^{+}, 168+$ pGP3306) and compared growth of the three 
strains in minimal medium with 0.1 or $5 \mathrm{mM}$ potassium (see Fig. 6.3a). All three strains grew very similar with $5 \mathrm{mM}$ potassium (growth rates of $0.43,0.39$, and $0.43 \mathrm{~h}^{-1}$ for the wild type, the dar $\mathrm{B}^{+}$, and the $\Delta$ darB mutant, respectively). In contrast, at the low potassium concentation, we observed a delayed growth for the strain overexpressing DarB as compared to the wild type and the darB deletion mutant ( 0.09 vs. 0.21 and $0.18 \mathrm{~h}^{-1}$, respectively). This is the condition when c-di-AMP is present in low amounts, and thus a large fraction of the DarB protein is present as apo-protein with the capacity to bind to Rel. It is therefore tempting to speculate that this interaction might be the reason for the growth defect. To test this idea, we deleted the rel gene in the wild type strain and in the strain carrying the expression vector for $\operatorname{dar} B$ and compared the growth in minimal medium at a low potassium concentration. As shown in Fig. 6.3b, the deletion of rel suppressed the growth defect that resulted from the overexpression of DarB (growth rates of 0.15 and $0.18 \mathrm{~h}^{-1}$ for the rel mutant and the rel mutant with overexpression of DarB, respectively). Overexpression of the DarB mutant proteins that are defective in the interaction with Rel did not result in growth inhibition (Fig. 6.3c). Taken together, all these observations indicate that the growth-inhibiting effect of DarB overexpression is the result of its interaction with Rel and suggests that DarB might control Rel activity.

Transcription of rRNA promoters is decreased under conditions of the stringent response (Murray et al., 2003; Krásný and Gourse, 2004). We observed that RNA extracted from the strain overexpressing DarB, in contrast to the wild type, lacked the rRNA intermediate migrating above the $16 \mathrm{~S}$ band (Supplementary Fig. 6.9). This band corresponds to the size of the pre-16S rRNA (Kurata et al., 2015). This decrease in the pre-16S rRNA pool most likely results from the reduced rRNA promoter activity and drainage of the pre-RNA pool. This suggests that the overexpression of DarB affects $r r n$ transcription by stimulation of (p)ppGpp synthesis in vivo. The downregulation of rRNA genes results in problems in ribosome assembly and might explain the observed Rel-dependent growth defect of the DarB overexpression strain during exponential growth.

\section{DarB controls Rel synthetase and hydrolase activities}

The results presented above suggest that the interaction between DarB and Rel might affect synthesis of (p)ppGpp by Rel. To test this idea, we used purified Rel protein to assay its synthetase and hydrolase activities. The purified Rel protein had little biosynthetic activity, as indicated by the production of 1.1 pmol pppGpp per pmol of Rel per minute (Fig. 6.4a). This is in good agreement with the absence of Rel synthetase activity if not triggered by uncharged tRNAs at the ribosome (Brown et al., 2016). In contrast, Rel activity was enhanced threefold if purified DarB protein was added to the assay mixture. If DarB was saturated with c-di-AMP prior to incubation with Rel, the Rel protein retained its background activity and no enhancement was detected. No pppGpp synthesis was detected with DarB 
alone indicating that DarB is unable to synthesize pppGpp and that the addition of DarB activates the synthetase activity of Rel. To exclude the possibility that the activation was just a result of non-specific protein crowding, we also assayed pppGpp synthesis by Rel in the presence of bovine serum albumin. In this case, Rel exhibited only background activity (see Fig. 6.4a). These results demonstrate that the interaction between apo-DarB and Rel stimutates the synthesis of pppGpp and that this stimulation is prevented in the presence of c-di-AMP.

A

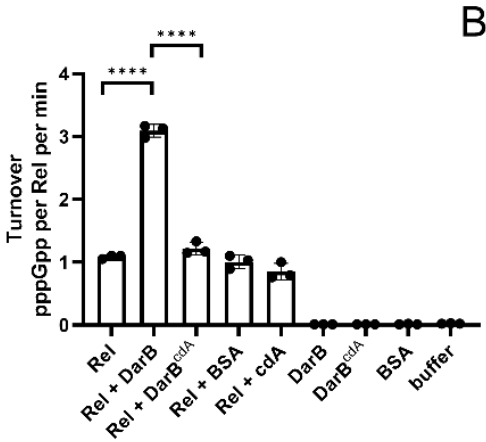

B

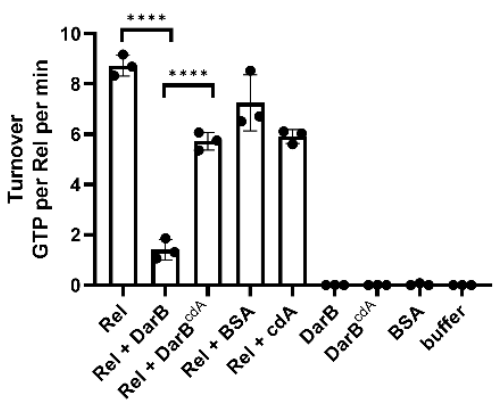

C

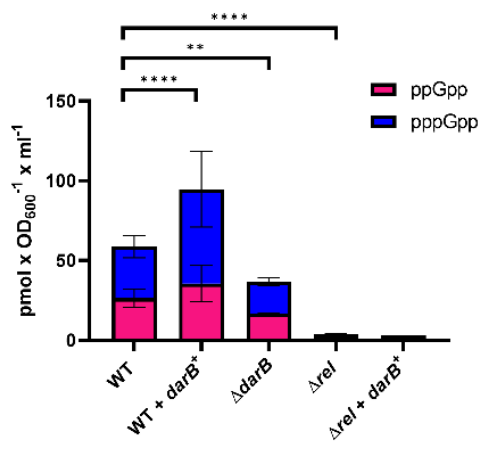

Fig. 6.4. DarB stimulates Rel-dependent accumulation of pppGpp. The activity of Rel was assessed in an in vitro activity assay. a, Rel synthetase activity assay. Purified Rel was incubated with ATP and GTP, in the absence or presence of DarB, C-di-AMP-saturated DarB, or bovine serum albumin (BSA) (10-fold molar excess) and the production of pppGpp was determined by liquid chromatography coupled tandem mass spectrometry on a QTRAP 5500 instrument (Sciex, Framingham, Massachusetts) equipped with an electrospray ionization source (ESI). BSA served as a negative control. Statistical analysis was performed using a one-way ANOVA, followed by Tukey's multiple comparisons test $(* * * * P<0.0001)$. $\mathbf{b}$, Rel hydrolase activity assay. Purified Rel was incubated with pppGpp, in the absence or presence of DarB, c-di-AMP-saturated DarB, or bovine serum albumin (BSA) (10-fold molar excess) and the production of GTP was monitored. c, Determination of intracellular (p)ppGpp levels in wild type B. subtilis, GP3407 ( $\Delta$ darB), wild type + pGP3306 (darB ${ }^{+}$), GP3419 ( $\Delta$ rel) and GP3419 + pGP3306 $\left(\Delta r e l, d a r B^{+}\right)$. Bacteria were grown in MSSM minimal medium with $0.1 \mathrm{mM} \mathrm{KCl}$ until the exponential growth phase, and the nucleotides were analyzed as decribed above. Abbreviation: cdA, c-di-AMP.

We also tested whether DarB affects the hydrolase activity of Rel. In this case, we determined the formation of GTP resulting from the hydrolysis of pppGpp. For the Rel protein alone, we determined a turnover rate of $8.7 \mathrm{pmol}$ of GTP per pmol of Rel per minute (Fig. 6.4b). In the presence of DarB, this activity was reduced six-fold. In the presence of c-di-AMP, DarB has little effect on the pppGpp hydrolase activity of Rel. As observed for the synthetase activity, these effects are specific since DarB has no pppGpp hydrolase activity, and the control protein (BSA) does not affect the hydrolytic activity of Rel. Taken together, these data demonstrate that DarB affects Rel activity by stimulation and inhibition of (p)ppGpp synthesis and degradation, respectively.

The data presented above suggest that the interaction of DarB with Rel results in a net increase of the intracellular ( $p$ )ppGpp levels. To verify this assumption, we compared the intracellular (p)ppGpp 
concentrations in a wild type strain, the $\operatorname{darB}^{+}$strain overexpressing DarB, and in the darB mutant (Fig. 6.4c). Indeed, overexpression of DarB resulted in a significant increase of (p)ppGpp (95 pmol $\mathrm{OD}_{600}{ }^{-1}$

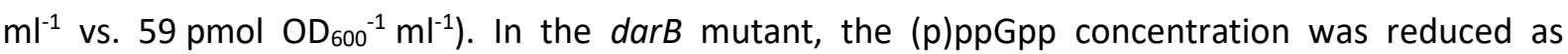
compared to the wild type strain. The determination of the (p)ppGpp concentration in a rel mutant strain revealed that Rel was the major source of $(p) p p G p p$ production under the conditions of our experiment, and that the residual ( $p$ )ppGpp synthesis was not affected by DarB overexpression. These observations are in agreement with the physiological observations. They confirm that the accumulation of (p)ppGpp is the cause of the growth inhibition of the strain that overexpresses DarB.

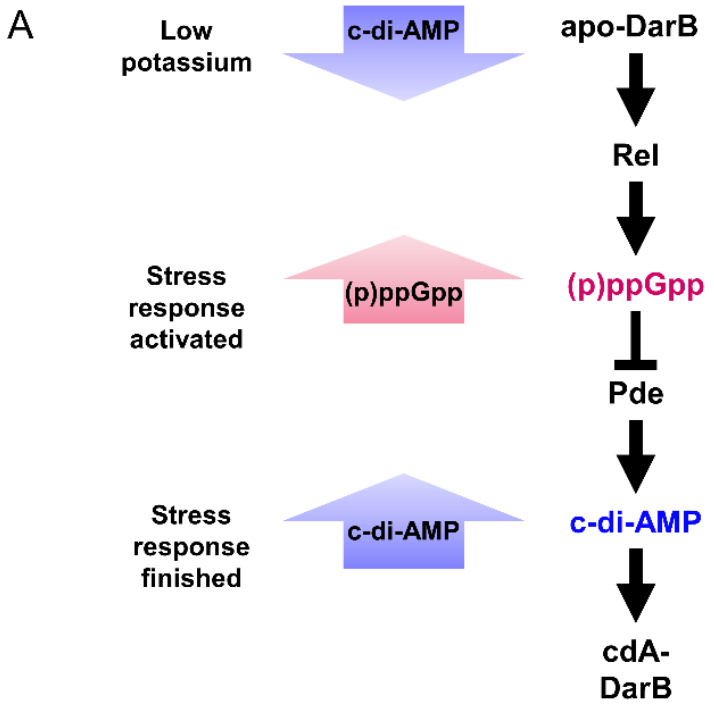

B

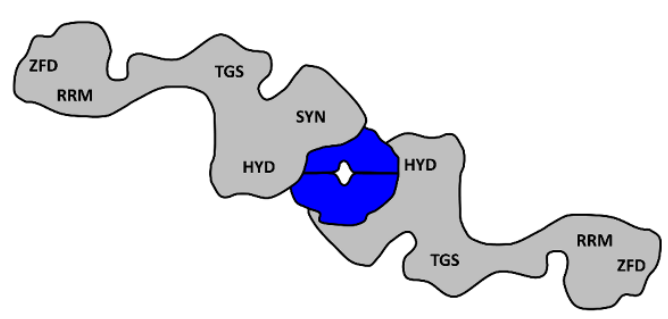

Fig. 6.5. The link between c-di-AMP and (p)ppGpp signaling in B. subtilis. (A) The model depicts the bidirectional and dynamic process of the cellular response to potassium limitation. When potassium becomes limiting, the diadenylate cyclases respond and produce less c-di-AMP and c-di-AMP receptor proteins are present in the apoform. Apo-DarB binds to Rel and stimulates (p)ppGpp synthesis. (p)ppGpp accumulation induces the stringent response and inhibits the c-di-AMP-degrading phosphodiesterases GdpP and $\mathrm{PgpH}$. This leads to increasing intracellular c-di-AMP amounts. DarB can then bind c-di-AMP and is thus no longer able to interact with Rel. (B) The DarB-Rel complex as suggested by the presented data. One DarB dimer is bound by two Rel monomers, one on each side. The interaction occurs via the HYD-SYN domains of Rel. DarB, blue; Rel, grey. Abbreviation: cdA, c-di-AMP. 


\section{DISCUSSION}

In this work, we report a novel link between potassium concentration, c-di-AMP signaling and the stringent response in the Gram-positive model organism B. subtilis. The observation that at low external potassium and intracellular c-di-AMP concentrations, the apo form of DarB binds to the alarmone synthetase Rel and triggers ( $p$ )ppGpp accumulation independent of the ribosome complements the earlier observations that on the other hand (p)ppGpp binds to the c-di-AMP degrading phosphodiesterases GdpP and PgpH to inhibit the degradation of c-di-AMP (Rao et al., 2010; Huynh et al., 2015; Corrigan et al., 2015). Together, these data allow to develop a model (Fig. 6.5a) in which at low potassium concentrations the intracellular c-di-AMP levels are low and the c-di-AMP targets including DarB are present in the apo form. DarB then binds to Rel and stimulates the synthesis of the alarmone (p)ppGpp in a ribosome-independent manner. The accumulation of ( $p$ )ppGpp results in a re-organisation of cellular physiology including the stop of translation. This direct link between the potassium concentration, the stringent response and ribosome activity is very important for the cell since potassium is essential for ribosome assembly and translation at the ribosome (Corrigan et al., 2016; Rozov et al., 2019). On the other hand, the accumulation of ( $p$ )ppGpp interferes with the degradation of c-di-AMP. This is likely to be important if potassium becomes available again. Then, c-di-AMP synthesis can be initiated and as long as ( $p$ )ppGpp is present, the second messenger is protected from degradation. This allows to achieve a c-di-AMP concentration that is appropriate to adjust the cellular potassium homeostasis by binding to c-di-AMP responsive riboswitches that control the expression of high affinity potassium transporters as well as to the potassium importers and exporters to inhibit and activate these proteins, respectively.

There is a huge body of evidence that ( $p$ )ppGpp synthesis by Rel is triggered by uncharged tRNA in the ribosomal A-site upon amino acid starvation (Hauryliuk et al., 2015; Brown et al., 2016). Our work supports the idea of ribosome-independent stimulation of the stringent response, as shown for phosphate and fatty acid starvation in E. coli (Germain et al., 2019). Our work now extends this concept also to potassium starvation in B. subtilis. Similar to the presence of uncharged tRNAs, a lack of potassium results in a stop of translation (Rozov et al., 2019), and does thus require similar global responses to reprogram translation, gene expression, DNA replication and cellular metabolism. It is interesting to note that c-di-AMP is a second messenger that reports on potassium availability in most Gram-positive and also in many Gram-negative bacteria with the notable exception of $\alpha-, \beta-$, and $\gamma$-proteobacteria (He et al., 2020; Stülke and Krüger, 2020). In $\alpha$ - and $\beta$-proteobacteria, a regulatory protein, PtsN (also referred to as enzyme IIA ${ }^{\mathrm{Ntr}}$ ) is capable of interacting with the single (p)ppGpp synthetase/hydrolase of these bacteria. The interaction depends on the nitrogen supply and the resulting phosphorylation state of PtsN and leads to the accumulation of (p)ppGpp (Karstens et al., 
2014; Ronneau et al., 2016; Ronneau et al., 2019b). PtsN has also been implicated in the control of potassium homeostasis: in E. coli, non-phosphorylated PtsN binds and inhibits TrkA, a subunit of lowaffinity potassium transporters as well as to the two-component sensor kinase $\mathrm{KdpD}$, thus stimulating its activity and the expression of the high-affinity Kdp potassium transport system (Lee et al., 2007; Lüttmann et al., 2009). It is thus intriguing to speculate that the regulatory link between potassium homeostasis and the stringent response is conserved in bacteria even though the specific molecular mechanisms may be completely different.

An interesting aspect of this study is the mode of DarB regulation by c-di-AMP. Our biophysical interaction analyses indicated an equimolar stoichiometry of the two proteins. Since DarB forms dimers, and the mutations that interfere with Rel binding are located at the upper and lower side of the DarB dimer, it is tempting to speculate that the proteins form a sandwich-like complex with a central DarB dimer and a molecule of Rel on each face of the dimer (see Fig. 6.5b). This resulting 2:2 stoichiometry is also best compatible with the results of the SEC-MALS analysis. Moreover, the differential affinities of DarB to c-di-AMP and Rel as well as the fact that two molecules of c-di-AMP bind to each DarB dimer in the region that is also important for Rel binding (Heidemann and Ficner, unpublished results) suggest that c-di-AMP and Rel compete for DarB binding. Since c-di-AMP has a 15-fold higher affinity for DarB than Rel, it is tempting to speculate c-di-AMP inhibits Rel binding in a competitive manner.

For all other processes controlled by c-di-AMP as well as other second messengers such as c-di-GMP, the nucleotide directly binds to its targets to control their activity, as has been shown for potassium uptake or export, osmolyte export, or pyruvate carboxylase activity (Sureka et al., 2014; Schuster et al., 2016; Huynh et al., 2016; Quintana et al., 2019; Gundlach et al., 2019). This raises the question why Rel needs DarB as a mediator of c-di-AMP mediated control. First, Rel is already composed of multiple domains, and it might have been difficult in evolution to integrate a further level of signaling directly into the protein. Second, potassium starvation is completely different from, but as serious for the cell as amino acid starvation. This makes it advantageous to have the two regulatory pathways for Rel activity separated from each other. Moreover, it is the apo form of DarB that binds and regulates Rel activity. An important function for apo-DarB has already been suggested by the observation that a $L$. monocytogenes mutant lacking c-di-AMP readily acquires mutations affecting the DarB counterpart CbpB (Whiteley et al., 2015). Similarly, mutations inactivating the DarA ortholog PstA were found in L. monocytogenes (Whiteley et al., 2015) suggesting that this protein might also interact with its partners in the apo form under conditions of potassium starvation.

DarB is conserved in several Gram-positive bacteria, including L. monocytogenes and Enterococcus faecalis. This suggests that the novel mode of control of the stringent response may also 
apply to these pathogens. In addition to DarB, B. subtilis Rel has been shown to interact with the competence protein ComGA, resulting in the inhibition of the hydrolase activity of Rel (Hahn et al., 2015). Moreover, a recent study demonstrated the transient accumulation of ( $p$ )ppGpp upon heat stress (Schäfer et al., 2020). It will be interesting to study whether yet additional factors may control Rel activity to trigger the stringent response under specific stress conditions.

\section{EXPERIMENTAL PROCEDURES}

\section{Strains, media and growth conditions}

E. coli DH5 $\alpha$ and Rosetta DE3 (Sambrook and Russell, 2001) were used for cloning and for the expression of recombinant proteins, respectively. All $B$. subtilis strains used in this study are derivatives of the laboratory strain 168. B. subtilis and E. coli were grown in Luria-Bertani (LB) or in sporulation (SP) medium (Sambrook and Russell, 2001; Kunst and Rapoport, 1995). For growth assays and the in vivo interaction experiments, B. subtilis was cultivated in MSSM medium (Gundlach et al., 2017b). In this medium $\mathrm{KH}_{2} \mathrm{PO}_{4}$ was replaced by $\mathrm{NaH}_{2} \mathrm{PO}_{4}$ and $\mathrm{KCl}$ was added as indicated. The media were supplemented with ampicillin $(100 \mu \mathrm{g} / \mathrm{ml})$, kanamycin $(50 \mu \mathrm{g} / \mathrm{ml})$, chloramphenicol $(5 \mu \mathrm{g} / \mathrm{ml})$, or erythromycin and lincomycin ( 2 and $25 \mu \mathrm{g} / \mathrm{ml}$, respectively) if required.

\section{Phenotypic characterization}

To assay growth of $B$. subtilis mutants at different potassium concentrations, the bacteria were inoculated in LB medium and precultured in MSSM medium with $0.1 \mathrm{mM} \mathrm{KCl}$. The cultures were grown until exponential phase, harvested, washed three times in MSSM basal salts solution before an optical density at $600 \mathrm{~nm}\left(\mathrm{OD}_{600}\right)$ was adjusted to 1.0. For growth analysis in liquid medium, the cells were used to inoculate a 96 well plate (Microtest Plate 96 Well, Sarstedt) containing MSSM medium with ammonium and the required potassium concentrations. Growth was tracked in an Epoch 2 Microplate Spectrophotometer (BioTek Instruments) at $37^{\circ} \mathrm{C}$ with linear shaking at $237 \mathrm{cpm} \mathrm{(4} \mathrm{mm)} \mathrm{for} 20 \mathrm{~h}$, and an $\mathrm{OD}_{600}$ was measured in 10 min intervals.

\section{DNA manipulation}

Transformation of E. coli and plasmid DNA extraction were performed using standard procedures (Sambrook and Russell, 2001). All commercially available plasmids, restriction enzymes, T4 DNA ligase and DNA polymerases were used as recommended by the manufacturers. Chromosomal DNA of B. subtilis was isolated as described (Kunst and Rapoport, 1995). B. subtilis was transformed with plasmid and genomic DNA according to the two-step protocol (Kunst and Rapoport, 1995). 
Introduction of mutations in the darB allele was achieved by the Combined Chain Reaction by using an additional 5' phosphorylated primer to introduce the mutation (Bi and Stambrook, 1998).

\section{Construction of mutant strains by allelic replacement}

Deletion of the darB and rel genes was achieved by transformation of $B$. subtilis 168 with a PCR product constructed using oligonucleotides to amplify DNA fragments flanking the target genes and an appropriate intervening resistance cassette as described previously (Lehnik-Habrink et al., 2011). The integrity of the regions flanking the integrated resistance cassette was verified by sequencing PCR products of about 1,100 bp amplified from chromosomal DNA of the resulting mutant strains, GP3409 and GP3419, respectively. Similarly, a strain allowing expression of Rel fused to C-terminal His-tag was constructed by first generating an appropriate PCR product and subsequent transformation of B. subtilis 168. The resulting strain was GP3429.

\section{Plasmid constructions}

The $c c p C$, darB, rel, sasA and sasB alleles were amplified using chromosomal DNA of $B$. subtilis 168 as the template and appropriate oligonucleotides that attached specific restriction sites to the fragment. Those were: Kpnl and BamHI for cloning rel in pGP172 (Merzbacher et al., 2004), BamHI and Sall for cloning rel in pWH844 (Schirmer et al., 1997), Xbal and Kpnl for cloning all genes in the BACTH vectors (Karimova et al., 1998), BamHI and Kpnl sites for cloning rel into pGP888 (Diethmaier et al., 2011) for genomic integration. The truncated rel variants were constructed as follows: rel-SYN-RRM contained aa 168-734, rel-HYD-SYN contained aa 1-391. For the overexpression of DarB, darB was amplified using chromosomal DNA of $B$. subtilis 168 as the template and appropriate nucleotides that attached Bsal and Xhol restriction sites to the fragments and cloned between the Bsal and Xhol sites of the expression vector pET-SUMO (Invitrogen, Germany). The resulting plasmid was pGP2972. All plasmids are listed in Supplementary Table 6.4.

\section{Protein expression and purification}

E. coli Rosetta(DE3) was transformed with the plasmid pGP2972, pGP3437, pGP3441, pGP3460 encoding wild type or mutant 6xHis-SUMO-DarB for purification of DarB or with the plasmids pGP3348 or pGP3350 for expression of Strep-tagged full-length Rel and Rel ${ }^{\mathrm{NTD}}$, respectively, or pGP706 (Blencke et al., 2006) for expression of 6xHis-CcpC. For purification of 10xHis-SUMO-Rel, pVHP186 (Takada et al., 2020) was transformed into E.coli Rosetta(DE3). Expression of the recombinant proteins was induced by the addition of isopropyl 1-thio- $\beta$-D-galactopyranoside (final concentration, $1 \mathrm{mM}$ ) to exponentially growing cultures $\left(\mathrm{OD}_{600}\right.$ of 0.8$)$ of E. coli carrying the relevant plasmid. His-tagged 
proteins were purified in $1 \times$ ZAP buffer ( $50 \mathrm{mM}$ Tris- $\mathrm{HCl}, 200 \mathrm{mM} \mathrm{NaCl}, \mathrm{pH} 7.5)$, if not stated otherwise, and Strep-tagged proteins in buffer W (100 mM Tris- $\mathrm{HCl}, 150 \mathrm{mM} \mathrm{NaCl}, 1 \mathrm{mM} \mathrm{Na}{ }_{2} \mathrm{EDTA}$, pH 8.0). 10xHis-SUMO-Rel was purified in buffer A ( $750 \mathrm{mM} \mathrm{KCl}, 5 \mathrm{mM} \mathrm{MgCl}, 40 \mu \mathrm{M} \mathrm{MnCl}_{2}, 40 \mu \mathrm{M}$ $\mathrm{Zn}(\mathrm{OAc})_{2}, 20 \mathrm{mM}$ imidazole, 10\% glycerol, 4 mM $\beta$-mercaptoethanol, $25 \mathrm{mM} \mathrm{HEPES}: \mathrm{KOH}$ pH 8) (Takada et al., 2020). Cells were lysed by four passes at 18,000 p.s.i. through an HTU DIGI-F press (G. Heinemann, Germany). After lysis, the crude extract was centrifuged at 100,000 x g for 60 min and then passed over a $\mathrm{Ni}^{2}+$ nitrilotriacetic acid column (IBA, Göttingen, Germany) for 6xHis-tagged and 10xHis-tagged proteins, or a StrepTactin column (IBA, Göttingen, Germany) for purification of Streptagged proteins. The protein was eluted with an imidazole gradient or D-desthiobiotin $(2.5 \mathrm{mM})$, respectively. After elution, the fractions were tested for the desired protein using SDS-PAGE. For the purification of Rel, the column was washed with 8 column volumes of $4 \mathrm{M} \mathrm{NaCl}$ to remove RNA prior to elution of the protein with $100 \mathrm{mM}$ and $250 \mathrm{mM}$ imidazole. To remove the SUMO tag from the proteins, the relevant fractions were combined, and the SUMO tag was removed with the SUMO protease (ratio 100:1) during overnight dialysis against 1 x ZAP buffer for DarB or against storage buffer $(720 \mathrm{mM} \mathrm{KCl}, 5 \mathrm{mM} \mathrm{MgCl}$, $50 \mathrm{mM}$ arginine, $50 \mathrm{mM}$ glutamic acid, $10 \%$ glycerol, $4 \mathrm{mM} \beta$ mercaptoethanol, 25 mM HEPES:KOH pH 8) (Takada et al., 2020) for Rel. The cleaved SUMO moiety and the protease were removed using a $\mathrm{Ni}^{2}+$ nitrilotriacetic acid column (IBA). The purified Rel was concentrated in a Vivaspin turbo 15 (Sartorius) centrifugal filter device (cut-off $50 \mathrm{kDa}$ ). The protein was loaded on a HiLoad 16/600 Superdex 200 pg column pre-equilibrated with storage buffer and the fractions containing pure Rel protein were collected and concentrated in a Vivaspin turbo 15 (Sartorius). The purity of protein preparations and the absence of RNA were assessed by SDS-PAGE and on a $1 \%$ agarose gel (in $1 \times$ TAE buffer; $40 \mathrm{mM}$ Tris-base, $1 \%$ acetic acid, $1 \mathrm{mM}$ EDTA pH 8.0), respectively. The protein samples were stored at $-80^{\circ} \mathrm{C}$ until further use (but no longer than 3 days). The protein concentration was determined according to the method of Bradford (Bradford, 1976) using the Bio-Rad dye binding assay and bovine serum albumin as the standard.

\section{Initial pulldown for identification of potential binding partners}

In order to identify potential binding partners of DarB, E. coli Rosetta (DE3) was transformed with pGP2972 (6xHis-SUMO-DarB) or the empty vector control pET-SUMO and the protein was overexpressed and purified as described above until the step where the protein was bound to the $\mathrm{Ni}^{2+}$ nitrilotriacetic acid column. After extensive washing, B. subtilis 168 crude extract (from LB) was added to the column to allow binding of $B$. subtilis proteins to the DarB protein (apo-DarB due to overexpression in E. coli). Again, after extensive washing, DarB, together with its potential binding 
partners, was eluted from the column with an imidazole gradient. The elution fractions from the eluates were subjected to mass spectrometry analysis.

\section{In vivo detection of protein-protein interactions}

To detect interaction partners of the DarB in vivo, cultures of B. subtilis GP3429 containing either pGP767 (DarB-Strep), or the empty vector control (pGP382), were cultivated in $500 \mathrm{ml}$ MSSM medium containing the indicated potassium concentrations until exponential growth phase was reached $\left(\mathrm{OD}_{600}\right.$ $\sim 0.4-0.6)$. The cells were harvested immediately and stored at $-20^{\circ} \mathrm{C}$. The Strep-tagged protein and its potential interaction partners were then purified from crude extracts using a StrepTactin column (IBA, Göttingen, Germany) and D-desthiobiotin as the eluent. The eluted proteins were separated on an SDS gel and potential interacting partners were analyzed by staining with Colloidal Coomassie and Western blot analysis. The eluents were further analyzed by mass spectrometry analysis.

\section{Protein identification by mass spectrometry}

Excised polyacrylamide gel pieces of protein bands were digested with trypsin as described previously (Shevchenko et al., 1996). Peptides were purified using C18 stop and go extraction (stage) tips as described (Rappsilber et al., 2003; Rappsilber et al., 2007). Dried peptide samples were reconstituted in $20 \mu$ l LC-MS sample buffer ( $2 \%$ acetonitrile, $0.1 \%$ formic acid). $2 \mu \mathrm{l}$ of each sample were subjected to reverse phase liquid chromatography for peptide separation using an RSLCnano Ultimate 3000 system (Thermo Fisher Scientific). Peptides were loaded on an Acclaim ${ }^{\circ}$ PepMap 100 pre-column

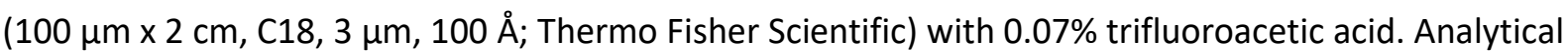
separation of peptides was done on an Acclaim "PepMap RSLC column $(75 \mu \mathrm{m} \times 50 \mathrm{~cm}, \mathrm{C} 18,3 \mu \mathrm{m}$, $100 \AA ̊$; Thermo Fisher Scientific) running a water-acetonitrile gradient at a flow rate of $300 \mathrm{nl} / \mathrm{min}$. All solvents and acids had Optima grade for LC-MS (Fisher Scientific). Chromatographically eluting peptides were on-line ionized by nano-electrospray (nESI) using the Nanospray Flex Ion Source (Thermo Scientific) and continuously transferred into the mass spectrometer ( $Q$ Exactive HF, Thermo Scientific). Full scans in a mass range of 300 to $1,650 \mathrm{~m} / \mathrm{z}$ were recorded with the $Q$ Exactive $H F$ at a resolution of 30,000 followed by data-dependent top $10 \mathrm{HCD}$ fragmentation at a resolution of 15,000 (dynamic exclusion enabled). LC-MS method programming and data acquisition was performed with the XCalibur software 4.0 (Thermo Fisher Scientific).

\section{LC-MS data analysis}

MS/MS2 data were searched against a B. subtilis specific protein database (UniProt Proteome ID UP000001570) using the Proteome Discoverer Software 2.2. The digestion mode was trypsin/P, and 
the maximum of missed cleavage sites was set two. Carbamidomethyl at cysteines was set as fixed modification, and oxidation at methionines and $\mathrm{N}$-terminal acetylation of proteins as variable modifications. Mass tolerances of precursors and fragment ions were $10 \mathrm{ppm}$ and $20 \mathrm{ppm}$, respectively. False discovery rates were calculated using the reverse-decoy mode, and the filter for peptide spectrum matches was set to 0.01 .

\section{In vitro analysis of protein-protein interactions}

To study the interaction between DarB and Rel, E. coli Rosetta (DE3) was transformed with pGP2972 (6xHis-SUMO-DarB), pGP3444 (6xHis-SUMO-DarB ${ }^{\text {A25G}), ~ p G P 3448 ~(6 x H i s-S U M O-D a r B ~}{ }^{\text {R132M }}$ ), pGP3460 (6xHis-SUMO-DarB'A25G,R132M), pGP3348 (Strep-Rel), or pGP3350 (Strep-Rel ${ }^{\mathrm{NTD}}$ ), respectively, and the proteins were overexpressed as described above. For purification of Rel or Rel ${ }^{\mathrm{NTD}}$, the crude extract was passed over a StrepTactin column (IBA, Göttingen, Germany) and washed with buffer W (pH 8.5) until the wash fractions appeared clear (confirmation with Bradford assay). Purified DarB, if stated preincubated 30 min with c-di-AMP (4x excess), or the control protein CcpC were added to the column, incubation happened overnight at $4^{\circ} \mathrm{C}$ under constant rotation. Purification was continued by extensive washing of the column with buffer W before Rel, together with binding partners, was eluted with D-desthiobiotin. For verification of the presence of DarB in the elution fractions, fixed and stained gel bands were excised and submitted to mass spectrometry analysis.

\section{SEC-MALS}

The interaction of the full-lenght Rel protein or Re ${ }^{\mathrm{NTD}}$ with DarB or DarB ${ }^{\mathrm{A} 25 G, R 132 \mathrm{M}}$ was analyzed by sizeexclusion chromatography and multi-angle light scattering (SEC-MALS). For this purpose, the purified tag-free proteins were either alone or pre-mixed in a 1:1 ratio in storage buffer (for full-lenght Rel) or $1 \times$ ZAP buffer (for Rel ${ }^{\mathrm{NTD}}$ ) ( $1 \mathrm{mg} / \mathrm{ml}$ each) applied onto the column. The buffer was filtered $(0.1 \mu \mathrm{m}$ filters) and degassed in line (Model 2003, Biotech AB/Sweden) prior to protein separation on a S200 Superdex 10/300GL column on an Äkta Purifier (both GE Healthcare). Subsequently, the eluate was analyzed in line with a miniDawn Treos multi angle light scattering system followed by an Optilab TrEX RI detector (both from Wyatt Technology, Europe) before fractionation. The elution fractions were analyzed with SDS-PAGE. Data analysis was performed using the ASTRA 6.1 software (Wyatt Technology) and also compared to a gel filtration standard (Bio-Rad). 
Determination of binding affinities and of the stoichiometry of the DarB-Rel ${ }^{\text {NTD }}$ complex by isothermal titration calorimetry

ITC experiments were carried out with a VP-ITC microcalorimeter (MicroCal Inc., Northampton, MA) in order to determine the affinity of DarB to Rel ${ }^{\text {NTD }}$ and the oligomerization state of the complex. In a typical setup, $\operatorname{Re}^{\mathrm{NTD}}(5 \mu \mathrm{M}$ in $50 \mathrm{mM}$ Tris- $\mathrm{HCl}, \mathrm{pH} 8.3,200 \mathrm{mM} \mathrm{NaCl}$ ) was placed in the sample cell, and DarB (100 $\mu \mathrm{M}$ in the same buffer) was placed in the titration syringe. All experiments were carried out at $20^{\circ} \mathrm{C}$ with and a stirring speed of $307 \mathrm{rpm}$. The parameters used for the titration series are given in Supplementary Table 6.3. Data analysis was carried out using MicroCal PEQ-ITC Analysis, Malvern Panalytical software.

\section{Bacterial two-hybrid assay}

Primary protein-protein interactions were identified by bacterial two-hybrid (BACTH) analysis (Karimova et al., 1998). The BACTH system is based on the interaction-mediated reconstruction of Bordetella pertussis adenylate cyclase (CyaA) activity in E. coli BTH101. Functional complementation between two fragments (T18 and T25) of CyaA as a consequence of the interaction between bait and prey molecules results in the synthesis of cAMP, which is monitored by measuring the $\beta$-galactosidase activity of the CAMP-CAP-dependent promoter of the E. coli lac operon. Plasmids pUT18C and p25N allow the expression of proteins fused to the T18 and T25 fragments of CyaA, respectively. For these experiments, we used the plasmids pGP2974-pGP2977, which encode N-and C-terminal fusions of T18 or T25 to darB. Accordingly, plasmids pGP2982-pGP2985 were used for ccpC, pGP3344-pGP3347 for rel, pGP3415-pGP3418 for rel(SYN-RRM), pGP3419-pGP3422 for rel(Rel ${ }^{\text {NTD }), ~ p G P 3336-p G P 3339 ~ f o r ~}$ sasA, and pGP3411-pGP3414 for sasB. These plasmids were used for co-transformation of E. coli BTH101 and the protein-protein interactions were then analyzed by plating the cells on LB plates containing $100 \mu \mathrm{g} / \mathrm{ml}$ ampicillin, $50 \mu \mathrm{g} / \mathrm{ml}$ kanamycin, 40 g/ml X-Gal (5-bromo-4-chloro-3-indolyl-ßD-galactopyranoside), and $0.5 \mathrm{mM}$ IPTG (isopropyl-ß-D-thiogalactopyranoside). The plates were incubated for a maximum of $36 \mathrm{~h}$ at $28^{\circ} \mathrm{C}$.

\section{Total RNA preparation}

For RNA isolation, the cells were grown in MSSM minimal medium containing $0.1 \mathrm{mM} \mathrm{KCl}$ to an $\mathrm{OD}_{600}$ of 0.4 to 0.6 and harvested. Preparation of total RNA was carried out as described previously (Meinken et al., 2003). RNA was visualized using a 1\% agarose formaldehyde gel in MOPS buffer (20 mM MOPS, $5 \mathrm{mM}$ Na-Acetate, $1 \mathrm{mM}$ EDTA, pH 7). The gel was stained with ethidium bromide. 


\section{Quantification of (p)ppGpp in B. subtilis cell extracts}

Bacteria were precultured in LB medium and in MSSM medium with $0.1 \mathrm{mM} \mathrm{KCl}$, and this preculture was used to inoculate the main culture in MSSM medium with $0.1 \mathrm{mM} \mathrm{KCl}$. The cultures were grown until the exponential phase. The nucleotides were extracted and quantified by the SPE Extraction (modified from Ihara et al., 2015). Briefly, $2 \mathrm{ml}$ of the culture were mixed with $75 \mu \mathrm{l} 100 \%$ formic acid and incubated on ice for $30 \mathrm{~min}$. After addition of $2 \mathrm{ml} 50 \mathrm{mM}$ ammonium acetate (pH 4.5), precipitates were removed by centrifugation at 3,000 $\mathrm{g}$ for $5 \mathrm{~min}$. The supernatant was transferred onto a prewashed SPE column (OASIS Wax cartridges $1 \mathrm{cc}$, Waters). Prewashing was done with $1 \mathrm{ml}$ methanol (4,500 x g for $1 \mathrm{~min}$ ) and $1 \mathrm{ml} 50 \mathrm{mM}$ ammonium acetate ( $\mathrm{pH} 4.5)$ (3,800 $\mathrm{xg}$ for $1 \mathrm{~min})$. The supernatant was loaded in $1 \mathrm{ml}$ steps ( 4 times) by 1 min centrifugation at 3,800 $\mathrm{x}$ g each time. The SPE

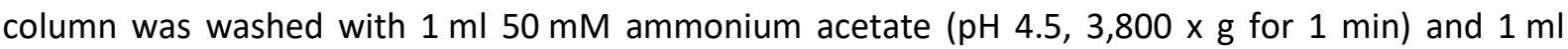
methanol (3,800 x g for $1 \mathrm{~min}$ ). After elution with $1 \mathrm{ml} 80 \% \mathrm{ddH}_{2} \mathrm{O}, 20 \%$ methanol, $3 \% \mathrm{NH}_{4} \mathrm{OH}$ into a new tube and centrifugation $(3,800 \times \mathrm{g}$ for $1 \mathrm{~min})$, the samples were frozen in liquid nitrogen and freeze-dried. The nucleotides were analyzed by liquid chromatography coupled tandem mass spectrometry on a QTRAP 5500 instrument (Sciex, Framingham, Massachusetts) equipped with an electrospray ionization source (ESI). Data were recorded in the multiple reaction monitoring (MRM) mode. Separation was performed on a Hypercarb column $(30 \times 4.6 \mathrm{~mm}, 5 \mu \mathrm{m}$ particle size; Phenomenex, Aschaffenburg, Germany ) using a linear gradient of solvent A (10 mM ammonium acetate $\mathrm{pH} \mathrm{10}$ ) and solvent $\mathrm{B}$ (acetonitrile) at a flow rate of $0.6 \mathrm{ml} / \mathrm{min}$, with solvent $B$ with a gradient of 4 to $60 \%$ B being delivered within 8 minutes. The ppGpp and pppGpp standards were purchased from Jena Bioscience.

\section{Rel synthetase activity assay}

Rel, and the wild type and A25G-R132M mutant DarB proteins were purified as described above. The assay was carried out in HEPES:Polymix buffer (20 mM HEPES:KOH pH 7.5, 2 mM DTT, 5 mM Mg(OAc)2, $95 \mathrm{mM} \mathrm{KCl}, 5 \mathrm{mM} \mathrm{NH}_{4} \mathrm{Cl}, 0.5 \mathrm{mM} \mathrm{CaCl}_{2}, 8 \mathrm{mM}$ putrescince, $1 \mathrm{mM}$ spermidine) (Takada et al., 2020). The activity of Rel was measured alone or in the presence of DarB, or the control protein BSA. The assay was carried out at $37^{\circ} \mathrm{C}$ and the reaction was started by addition of $1 \mathrm{mM}$ ATP and $1 \mathrm{mM}$ GTP (Jena Bioscience), and samples for the nucleotide measurement were taken after $15 \mathrm{~min}$. The nucleotides were extracted and quantified by SPE Extraction modified from Ihara et al. as decribed above, with the exception that $500 \mu \mathrm{l}$ of the assay mix was mixed with 1,500 $\mu$ lassay buffer and with $75 \mu \mathrm{l} 100 \%$ formic acid and incubated on ice for $30 \mathrm{~min}$. 


\section{Rel hydrolase activity assay}

The pppGpp hydrolysis assay was carried out in HEPES:Polymix buffer (20 mM HEPES:KOH pH 7.5, $2 \mathrm{mM}$ DTT, $5 \mathrm{mM} \mathrm{Mg}(\mathrm{OAC})_{2}, 95 \mathrm{mM} \mathrm{KCl}, 5 \mathrm{mM} \mathrm{NH}{ }_{4} \mathrm{Cl}, 0.5 \mathrm{mM} \mathrm{CaCl}, 8 \mathrm{mM}$ putrescince, $1 \mathrm{mM}$ spermidine), $1 \mathrm{mM} \mathrm{MnCl}$ ) (Takada et al., 2020). The activity of Rel was measured alone or in the presence of DarB, or the control protein BSA. The assay was carried out at $37^{\circ} \mathrm{C}$ and the reaction was started by addition of $1 \mathrm{mM}$ pppGpp (Jena Bioscience), and samples for the nucleotide measurement were taken after $15 \mathrm{~min}$. The nucleotides were extracted and quantified as decribed above.

\section{SUPPLEMENTARY MATERIAL}

https://www.biorxiv.org/content/10.1101/2020.08.27.268672v1

S6.1 Table Analysis of eluates from the DarB pulldown experiment with Bacillus subtilis cell extract.

S6.2 Table Analysis of excised gel bands from the in vitro pulldown experiment with Strep-Rel.

S6.3 Table Analysis of the in vivo interaction experiment of DarB-Strep with low $(0.1 \mathrm{mM})$ and high (5 $\mathrm{mM})$ potassium concentrations.

S6.4 Table Plasmids used in this study.

Fig. S6.1. $\quad$ DarB does not interact with $\mathrm{CcpC}$.

Fig. S6.2. Purification of Rel.

Fig. S6.3. Analysis of the DarB-Rel-complex by size exclusion chromatography (SEC).

Fig. S6.4. Analysis of the binding affinities of DarB, and the DarB mutants towards C-di-AMP and Rel ${ }^{\text {NTD }}$.

Fig. S6.5. In vitro interaction experiment between DarB and Strep-Rel or Strep-Rel ITD.

Fig. S6.6. In vitro interaction experiment between DarB, $\operatorname{DarB}^{\mathrm{A25G}}, \operatorname{DarB}^{\mathrm{R} 132 \mathrm{M}}, \operatorname{DarB}^{\mathrm{A} 25 \mathrm{G}, \mathrm{R} 132 \mathrm{M}}$, and Strep-Rel.

Fig. S6.7. DarB does not interact with the small alarmone synthetases SasA and SasB.

Fig. S6.8. In vitro analysis of the DarB-Rel ${ }^{\mathrm{NTD}}$ complex.

Fig. S6.9. $\quad$ Analysis of rRNA profiles in B. subtilis mutants.

\section{DATA AVAILABILITY}

The mass spectrometry proteomics data have been deposited to the ProteomeXchange Consortium via the PRIDE (Perez-Riverol et al., 2019) partner repository with the dataset identifier PXD018087.

\section{ACKNOWLEDGEMENTS}


We wish to thank Vasili Hauryliuk for providing an expression vector and a detailed protocol for the purification of $B$. subtilis Rel. We are grateful to Oliver Valerius for the help with LCMS analyses which were done at the Service Unit LCMS Protein Analytics of the Göttingen Center for Molecular Biosciences (GZMB) at the GeorgAugust-University Göttingen (Grant ZUK 41/1 DFG-GZ A 630 to G.H. Braus and grant DFG-GZ: INST 186/1230-1 FUGG to S. Pöggeler). We wish to thank Gabriele Beyer, Mats Koschel, and Tobias Krammer for helpful discussions and technical assistance. Annette Garbe is acknowledged for the nucleotide analysis. This work was supported by grants of the Deutsche Forschungsgemeinschaft (DFG) within the Priority Program SPP1879 (to R.F. and J.S.) and INST186/1117 (to R.F.). 


\section{Chapter 7 - Discussion}

\section{1 c-di-AMP and its role in maintaining potassium homeostasis}

Cells of any kind lack the ability to actively transport water. Thus, the cellular hydration status is determined by the transport of ions, driven by the basic principle of osmosis (Wood, 1999; Wood, 2011). Hence, even minor changes in the external salinity impact the turgor and consequently affect the water content of the cell. B. subtilis, as a soil inhabitant, has to adapt to rapidly changing osmotic conditions, that are caused by both daily and seasonal changes in the weather that highly affect the hydration, and consequently the osmotic status of the soil. The response to these osmotic changes represents one of the most critical issues in the lifecyle of bacteria, and the tight regulation of the responsive pathways is of particular importance to guarantee survival (Bremer and Krämer, 2019). Upon a sudden osmotic upshift (increase in extracellular osmolality), bacteria respond in a two-step process of adaption. To prevent immediate water efflux, the cell first accumulates up to $700 \mathrm{mM}$ potassium (Dinnbier et al., 1988; Whatmore et al., 1990; Booth and Higgins, 1990; Wood, 1999; Epstein, 2003; Bremer and Krämer, 2019). This leads to an immense increase of ionic strength of the cytoplasm. In the second step potassium ions are replaced by compatible solutes like glycine betaine or proline, which can be imported or synthesized de novo (Whatmore et al., 1990; Shannon et al., 2003; Brill et al., 2011; Zaprasis et al., 2015; Bremer and Krämer, 2019). This decreases the ionic strength and improves the solubility of the cytoplasm. The prior uptake of potassium ions is essential for stimulation of the synthesis of osmoprotectants like proline (Whatmore et al., 1990). It has been proposed that potassium stimulates the uptake as well as the synthesis of proline (Dinnbier et al., 1988). This can be explained by the stimulatory effect of potassium on the glutamate dehydrogenase Gdh that increases the metabolic flux towards proline synthesis (Measures, 1975). Thus, the accumulation of potassium ions during an osmotic upshift is accompanied by an increase in glutamate synthesis. Similarly, an increase in expression of the glutamate synthase gltAB has been observed in B. subtilis in this study (see Chapter 5). Since glutamate is negatively charged at a neutral $\mathrm{pH}$, potassium ions accumulate to counterbalance the negative charge, resulting in a cyclic build-up of both potassium and glutamate (Measures, 1975). Furthermore, glutamate itself serves as a direct precursor for synthesis of the osmoprotectant proline. Since proline is an uncharged molecule, no concomitant accumulation of another ion is required and the cell has a long-term protection against high external osmotic conditions, without a critical increase in the ionic strength of the cytoplasm.

The initial response to an osmotic upshift requires the tight regulation of the potassium uptake systems. B. subtilis encodes three importers: the two potassium channels $K \operatorname{tr} A B$ and $K \operatorname{trCD}$, and the potassium transporter KimA. In this work we have demonstrated binding of c-di-AMP to the recently 
identified KimA protein (see Chapter 3; Gundlach et al., 2017b; Gundlach et al., 2019). Additionally, we identified a novel regulatory mechanism for the KtrCD channel, that links potassium homeostasis and c-di-AMP signaling to nitrogen metabolism.

KtrCD and KtrAB are potassium channels of the Ktr/Trk type (Holtmann et al., 2003). Both channels are inhibited by c-di-AMP, which binds to the RCK_C domain of the cytoplasmic subunits KtrC and KtrA (Corrigan et al., 2013; Kim et al., 2015; Rocha et al., 2019; Gundlach et al., 2019). The $K_{D}$ for c-di-AMP binding has been described as $30 \mathrm{nM}$ and $3 \mu \mathrm{M}$, for KtrC and KtrA, respectively (Rocha et al., 2019). The 100-fold higher affinity of KtrC for c-di-AMP is quite remarkable and immediately questions the physiological relevance of this potassium channel in actual import of the ion. The intracellular c-di-AMP concentrations range between $1.2 \mu \mathrm{M}$ and $5.1 \mu \mathrm{M}$ during vegetative growth and $2 \mathrm{~h}$ after sporulation, respectively (Oppenheimer-Shaanan et al., 2011). One critical point in the common determination of intracellular nucleotide pools is the assumption that bacterial cultures are homogeneous when in reality a huge heterogeneity is displayed (Kelly and Rahn, 1931; Elfwing et al., 2004; Strovas et al., 2007). Even if a cell culture for nucleotide analysis is harvested at a specific $\mathrm{OD}_{600}$, the cells differ greatly in their growth phase and cell fate/morphology. As the cell grows and divides, fluctuations in the nucleotide pool are very likely. Nucleotides measured in a culture always correspond to the average of all cells. The concentration of $1.2 \mu \mathrm{M}$ c-di-AMP in cells during vegetative growth (Oppenheimer-Shaanan et al., 2011), has to be regarded as the mean of the culture, rather than the nucleotide content of a single cell. Thus, it appears likely, that the minimal c-di-AMP concentration can actually be lower than $1 \mu \mathrm{M}$. Another issue is the nucleotide extraction procedure (Gundlach et al., 2015b), not only free nucleotides are determined, but also bound nucleotides, that actually do not contribute to the free nucleotide pool of the cytoplasm. In order to make more accurate predictions about the intracellular pool of the respective second messenger, the heterogeneity of a cell culture should be taken into consideration, and the extraction should be reassessed in the future.

The affinity studies of KtrC and c-di-AMP were performed without the transmembrane protein KtrD (Rocha et al., 2019). This, together with the general difficulties of assuming physiological conditions from in vitro data, could lead to a lower actual affinity of KtrC towards its inhibitor. Nevertheless, even if the affinity for c-di-AMP is lower in reality, the extremely low $K_{D}$ still suggests the possibility that KtrCD is mostly inhibited under physiological c-di-AMP concentrations. The physiological concentration of KtrC ranges between 0.5 and $6 \mu \mathrm{M}$ (Maaß et al., 2014; Zhu and Stülke, 2018). The physiological concentration of the transmembrane partner KtrD has not been determined so far, but based on the expression data (Zhu and Stülke, 2018), it can be assumed that the amount of protein is lower than KtrC. Accordingly, there should always be an excess of KtrC over KtrD, which could allow formation of Apo-KtrCD complexes even if a specific number of KtrC proteins is bound to 
c-di-AMP. In addition to the fact that the intracellular concentration of $\mathrm{KtrC}$ is in the range of the nucleotide, one c-di-AMP molecule blocks one KtrC dimer (Rocha et al., 2019), increasing the potential cases of inhibition of KtrC by c-di-AMP even further. Hence, it appears likely, that inhibition of KtrCD by the second messenger is the rule rather than the exception. KtrA on the other hand, binds c-di-AMP with an affinity of $3 \mu \mathrm{M}$ (Rocha et al., 2019), which corresponds to the physiological c-di-AMP concentration (Oppenheimer-Shaanan et al., 2011). Thus, under conditions of lower c-di-AMP levels (low extracellular potassium concentrations), KtrA would be present in the apo-form and potassium import via KtrAB would be possible because (I) both expression of $k \operatorname{tr} A B$, and KtrAB are not inhibited by c-di-AMP, and (II) KtrAB, as a high-affinity potassium channel (Holtmann et al., 2003), is able to import potassium ions when the external concentration of the cation is low. This suggests a completely different mode of action for KtrA and KtrC, and raises the question how bacteria like S. aureus, which encode two transmembrane proteins KtrB and KtrD but only one cytosolic partner, that is closer related to KtrC than KtrA, regulate potassium uptake in detail (Gries et al., 2013; Price-Whelan et al., 2013; Gründling, 2013). While in S. aureus KtrCB and KtrCD are involved in low-affinity potassium uptake, it has recently been described for the $B$. subtilis Ktr system, that the affinity of the transporter is determined by the transmembrane domain, and that the cyctosolic domains are interchangeable without affecting the affinity of the gating subunit (Price-Whelan et al., 2013; Rocha et al., 2019). Whether the transmembrane protein KtrB is regulated differently in S. aureus and B. subtilis, or why $\mathrm{KtrCB}^{\text {S. aureus }}$ shows low affinity for potassium compared to $\mathrm{KtrCB}^{\text {B. subtilis }}$, remains to be answered in the future.

The high affinity of KtrCD for c-di-AMP suggests that the potassium channel is active only at extremely low c-di-AMP levels. So far the only condition known to cause such low levels, is low external potassium availability (Gundlach et al., 2017b). Since KtrCD has previously been described as a lowaffinity potassium channel (Holtmann et al., 2003), the channel would be unable to import the cation under these conditions. However, in this work, we discovered a novel regulatory mechanism of the potassium channel KtrCD that changes the affinity of the channel towards its substrate (see Chapter 4; Krüger et al., 2020b). We observed a 16-fold increase in the affinity of KtrCD for potassium in the presence of glutamate compared to the presence of ammonium (see Chapter 4; Krüger et al., 2020b). Since the intracellular amounts of potassium and glutamate need to be balanced, this regulatory circuit displays an additional level of regulation that allows the cell to respond to the availability of glutamate by stimulated import of potassium ions. This regulation is of particular importance given the fact that KtrCD is the most strongly expressed potassium transporter in B. subtilis (Zhu and Stülke, 2018). This increase in affinity for potassium in the presence of glutamate, when potassium levels are low, would display one of the few conditions, in which (I) c-di-AMP levels are low enough to not inhibit KtrCD, and 
(II) KtrCD is indeed able to bind and import the ion. As glutamate is known to affect the intracellular c-di-AMP levels (Gundlach et al., 2015b), it is of crucial importance, that once glutamate is available, the accumulation of potassium via KtrCD is not limited by the channels affinity for the ion. This gives a complete new perspective on the relevance of KtrCD under physiological conditions and it will be interesting to study the potentially counteracting effects of c-di-AMP and glutamate on KtrCD in the future.

KimA belongs to the Kup/HAK/KT ( $\mathrm{K}^{+}$Uptake Permease, Kup) family, a second class of potassium transporters next to the Ktr family (Tascón et al., 2020). Members of the Kup family belong to the APC (amino acid-polyamine-organocation) superfamily and members of this superfamily accumulate the substrate by the symport of a cation (Vastermark et al., 2014; Tascón et al., 2020). Thus, the activity of the transporter is higher under acidic environmental conditions. The activity of KimA is inhibited by binding of c-di-AMP to the C-terminal cytoplasmic domain (see Chapter 3; Gundlach et al., 2019; Gibhardt et al., 2019). Similarly, inhibition of c-di-AMP has been observed for two members of the Kup family, the high-affinity potassium transporters KupA and KupB, in L. lactis (Quintana et al., 2019).

The ability of a single signaling molecule to control one biological process at different stages is termed "Sustained Sensing" and ensures tight regulation of the respective process (Orr et al., 2016). This implies the involvement of more than one receptor, that recognizes the same second messenger, in the same process. c-di-AMP is able to control potassium homeostasis on transcriptional and on protein level (Gundlach et al., 2017b; Gundlach et al., 2019). On transcriptional level, c-di-AMP binds the $y d a O$ riboswitch, which is located in the 5'UTRs of the the kimA gene and the ktrAB operon and inhibits expression of the high-affinity potassium transporters (Nelson et al., 2013; Gundlach et al., 2017b). On protein level, c-di-AMP binds KimA and KtrA, and prevents potassium uptake when intracellular potassium levels increase (Kim et al., 2015; Rocha et al., 2019; Gundlach et al., 2019). This ensures that the high-affinity potassium transporters are only expressed and functional under low potassium levels. While the affinity of KimA for c-di-AMP could not be determinded so far, c-di-AMP binds the $y d a O$ riboswitch very tight and the $\mathrm{K}_{\mathrm{D}}$ for the nucleotide binding is in the low $\mathrm{nM}$ range (10-66 nM) (Nelson et al., 2013; Gao and Serganov, 2014; Ren and Patel, 2014). This raises the immediate question, which physiological relevance this transporter has, considering c-di-AMP concentrations of the cytoplasm range between 1 and $5 \mu \mathrm{M}$ (Oppenheimer-Shaanan et al., 2011). This implies that the intracellular level of the nucleotide has to be below this range to allow expression of the transporter. Since changes in the availability of potassium indeed affect intracellular c-di-AMP levels, an increase from 0.1 to $5 \mathrm{mM} \mathrm{KCl}$ in the medium induced a two-fold increase in the nucleotide pool (Gundlach et al., 2017b). This is in agreement with the observation, that the availability of 
potassium impacts the $y d a O$ riboswitch and expression of the downstream kimA gene only occurrs at low external potassium concentrations (Gundlach et al., 2017b). The fact that bacteria encode several potassium uptake systems, demonstrates the need to fine-tune potassium uptake.

\subsection{The connection between c-di-AMP, potassium and glutamate}

In this study we observed that a strain lacking c-di-AMP is not only sensitive to potassium (Gundlach et al., 2017b) but also to glutamate (Chapter 5). The toxic accumulation of amino acids has also been observed in L. monocytogenes and S. aureus (Whiteley et al., 2015; Zeden et al., 2018; Zeden et al., 2020). This toxicity could be bypassed by the acquisition of loss-of-function mutations in oligopeptide or glutamine transporters, indicating that sensitivity to amino acids happens to be an universal problem without c-di-AMP (Whiteley et al., 2015; Zeden et al., 2018; Zeden et al., 2020). The link between nitrogen metabolism and c-di-AMP signaling was initially reported in B. subtilis, and has recently also been observed in S. aureus (Gundlach et al., 2015b; Zeden et al., 2020). The direct influence of each nitrogen source on c-di-AMP homeostasis, however, seems to differ between both species. While glutamate was observed to induce higher intracellular c-di-AMP levels in B. subtilis, no such increase was found in S. aureus (Gundlach et al., 2015b; Zeden et al., 2020). In fact, the presence of glutamine and ammonium led to a decrease in the intracellular concentration of the second messenger (Zeden et al., 2020). The increase of intracellular c-di-AMP levels in the presence of glutamate indicates that c-di-AMP is important for the adaptation to glutamate and that B. subtilis responds to the available nitrogen source via another so far unknown mechanism. Because of the correlation between intracellular potassium and glutamate levels (Measures, 1975), the depletion of the second messenger becomes harmful when the cell has to adapt to environments with high external potassium or glutamate concentrations (see Chapter 5; Gundlach et al., 2017b; Krüger et al., 2020a). Since intracellular c-di-AMP levels drop when S. aureus grows with glutamine, it is unclear why glutamine becomes toxic in a $\Delta d a c$ strain (Zeden et al., 2020). The strong correlation between potassium and glutamate can be observed by the stimulation of glutamate transporters by potassium ions in Streptococcus mutans and L. lactis (Poolman et al., 1987; Sato et al., 1989). Moreover, in B. subtilis the potassium exporter YugO is known to be stimulated upon glutamate limitation (Prindle et al., 2015). The importance of coordinated potassium and glutamate acquisition is further supported by the novel findings of this study that demonstrate the stimulation of the potassium channel KtrCD by glutamate, and vice versa the stimulation of the glutamate transporter GItT by potassium (see Chapter 4, 5; Krüger et al., 2020b). Similarly, the expression of kimA and gltA is regulated by glutamate and potassium, respectively (see Chapter 5 ). The interdepence of potassium and glutamate can be explained by their nature to function as counterions to balance the osmotic charge of the cytoplasm, 
and this is underlined by the observed effects of each ion on the transport and/or synthesis of the other.

c-di-AMP appears to be the regulating link between potassium and glutamate homeostasis. The interdependence between potassium homeostasis and nitrogen metabolism has been observed before and seems to be a conserved feature that allows the cell to keep the intracellular balance between the most abundant ions (see above). The canonical (PEP):carbohydrate phosphotransferase system (PTS) is used by many bacteria to coordinate carbohydrate uptake (Kundig et al., 1964; Bramley and Kornberg, 1987; Postma et al., 1993; Stülke and Hillen, 1998). The PTS system is a phosphorylation chain that consists of the three enzymes El, HPr, and Ell (Fig. 7.1; Postma et al., 1993). The phosphate group derives from phosphoenolpyruvate and is transferred to the imported sugar via this chain. Interestingly, many proteobacteria encode a second type of PTS system, the PTS ${ }^{\mathrm{Ntr}}$ system, that has exclusively regulatory functions (Lüttmann et al., 2009; Pflüger-Grau and Görke, 2010). The EllA ${ }^{\text {Ntr }}$ protein of E. coli for instance is involved in the regulation of potassium homeostasis (Fig. 7.1; Lüttmann et al., 2009; Lüttmann et al., 2015). Unphosphorylated EllA ${ }^{\mathrm{Ntr}}$ interacts with the low-affinity potassium transporter TrkA, thereby inhibiting potassium accumulation (Lee et al., 2007). Similarly, unphosphorylated EIIA ${ }^{\text {Ntr }}$ stimulates KdpD activity (Lüttmann et al., 2009). This leads in turn to increased KdpE phosphorylation that activates expression of the $k d p F A B C D$ operon, encoding a highaffinity potassium uptake system (Lüttmann et al., 2009). Under nitrogen limitation, $\alpha$-ketoglutarate, as the direct carbon donor used for $\mathrm{N}$-fixation, prevents phosphorylation of El of the canonical PTS system (EI) (Doucette et al., 2011). This leads to inactivation of the PTS system through accumulation of non-phosphorylated EI, HPr, and ElIA. However, because of the crosstalk between the canonical PTS and the PTS ${ }^{\mathrm{Ntr}}$ system, the phosphorylation status of ElIA ${ }^{\mathrm{Ntr}}$ is likely affected by that as well (Zimmer et al., 2008; Lüttmann et al., 2015). In fact, ElIA ${ }^{\mathrm{Ntr}}$ is phosphorylated by both $\mathrm{El}$ and $\mathrm{EI}^{\mathrm{Ntr}}$ in vitro and in vivo (Powell et al., 1995; Rabus et al., 1999; Zimmer et al., 2008; Pflüger-Grau and de Lorenzo, 2014). This idea is further supported by the absence of $\mathrm{El}^{\mathrm{Ntr}}$ and $\mathrm{NPr}$ in several $\beta$-proteobacteria like Ralstonia eutropha (Pflüger-Grau and Görke, 2010). In this organism, ElIA ${ }^{\mathrm{Ntr}}$ is phosphorylated solely by the canonical El and HPr enzymes (Krauße et al., 2009). In contrast to El that is inhibited by $\alpha$-ketoglutarate, $\mathrm{EI}^{\mathrm{Ntr}}$ shows the opposite reaction and its autophosphorylation is stimulated by the accumulation of $\alpha$ ketoglutarate and inhibited by glutamine, as shown by in vitro studies (Lee et al., 2013; Goodwin and Gage, 2014; Ronneau et al., 2016). Binding of $\alpha$-ketoglutarate to $\mathrm{El}^{\mathrm{Ntr}}$ requires the presence of the GAF domain, which is absent in El (Lee et al., 2013). Because of the opposite effects of $\alpha$-ketoglutarate on $\mathrm{EI}$ and $\mathrm{EI}^{\mathrm{Ntr}}$, finding new inhibitory factors for $\mathrm{EI}^{\mathrm{Ntr}}$ will be important, as is unveiling which of the two proteins acts as the major phosphoryl-group donor for EIIA ${ }^{\mathrm{NtrA}}$ in vivo in organisms like E. coli that encode both systems. Determination of the exact physiological role of $\mathrm{El}$ and $\mathrm{EI}{ }^{\mathrm{Ntr}}$ in phosphorylation 
of EllA ${ }^{\mathrm{Ntr}}$ will shed light on the phosphorylation status of EllA ${ }^{\mathrm{Ntr}}$ in E. coli upon nitrogen limitation. Knowledge of this would allow a deeper insight in the coordinated response to potassium and nitrogen limitation.

The c-di-AMP and PTS ${ }^{\text {Ntr }}$ signal transduction pathways are analogous in their function as regulators of potassium homeostasis (Fig 7.1). In fact, the toxic accumulation of potassium can be observed for both $\Delta d a c$ in B. subtilis and $\Delta p t s N$ (EllA ${ }^{\mathrm{Ntr}}$ ) in E. coli (Lüttmann et al., 2015; Gundlach et al., 2017b). While the PTS ${ }^{\mathrm{Ntr}}$ is able to integrate information about the nitrogen status of the cell via El/EI ${ }^{\mathrm{Ntr}}$ (Doucette et al., 2011; Lee et al., 2013), such an effector is still missing for the nucleotide second messenger pathway. Nevertheless, the observation of several nitrogen source related phenotypes in the context of c-di-AMP depletion in previous and present studies strongly indicates that such a direct link also exists here (see Chapter 4, 5; Whiteley et al., 2015; Gundlach et al., 2015b; Krüger et al., 2020a; Zeden et al., 2020). One hypothesis that is hard to dismiss is that the second signal transduction protein DarA is involved in sensing the nitrogen status of the cell. As a PII-like protein, it is highly likely to fullfil such function. Moreover, the interaction of PII proteins with other proteins upon ligand binding is a common observation (Ninfa and Jiang, 2005; Forchhammer and Lüddecke, 2016). PII proteins are often controlled by posttranslational modifications, like uridylylation or adenylylation (Merrick, 2015). PII proteins of some Cyanobacteria are modified by phosphorylation, as for instance GInB from Synechococcus elongatus (Forchhammer and Tandeau de Marsac, 1995; Forchhammer et al., 2004; Merrick, 2015). However, posttranslational modification of PII or PII-like proteins has so far not been observed in Firmicutes (Merrick, 2015). Yet, the adaptation of the $\Delta d a c$ strain to complex medium was only possible upon the deletion of DarA or DarB (see Chapter 5). The isolated suppressors gained mutations that led to a reduced uptake of potassium ions, indicating that the control of potassium homeostasis is indeed one of the main functions of c-di-AMP. In addition, suppressors isolated from the $\Delta d a c \Delta d a r B$ strain acquired further loss-of-function mutations in DarA, pointing to the toxicity and likewise importance of the apo-protein. This has also been observed in L. monocytogenes, where the c-di-AMP toxicity could be bypassed by loss-of-function mutations in CbpB/DarB, PstA/DarA, Rel, PycA, and CitZ (Whiteley et al., 2015; Whiteley et al., 2017). While mutations in PycA, CitZ, and PstA prevented otherwise toxic accumulation of citrate without c-di-AMP and suppressed the sensitivity to $\beta$-lactam antibiotics, mutations in $\mathrm{CbpB} / \mathrm{DarB}$ and Rel did not (Whiteley et al., 2017). This indicates that mutations in PstA/DarA, PycA and CitZ affect the same metabolic pathway. That is further supported by the parallel occurance of PstA/DarA mutations with mutations in CbpB/DarB and Rel, but not PycA and CitZ (Whiteley et al., 2015). Moreover, PstA/DarA mutations were always accompanied by mutations related to ion transport and osmoprotection (Whiteley et al., 2015), which is consistent with our findings (see Chapter 5), pointing to the involvment 
in another metabolic function. The above mentioned reasons point in a direction where DarA is involved in the regulation of the TCA-cylce, most likely at the intersection between carbon and nitrogen metabolism. In remains to be elucidated if DarA is indeed able to sense the nitrogen status of the cell, if it might be subject to posttranslational modification, and if the protein directly connects c-di-AMP signaling with the response to nitrogen limitation.
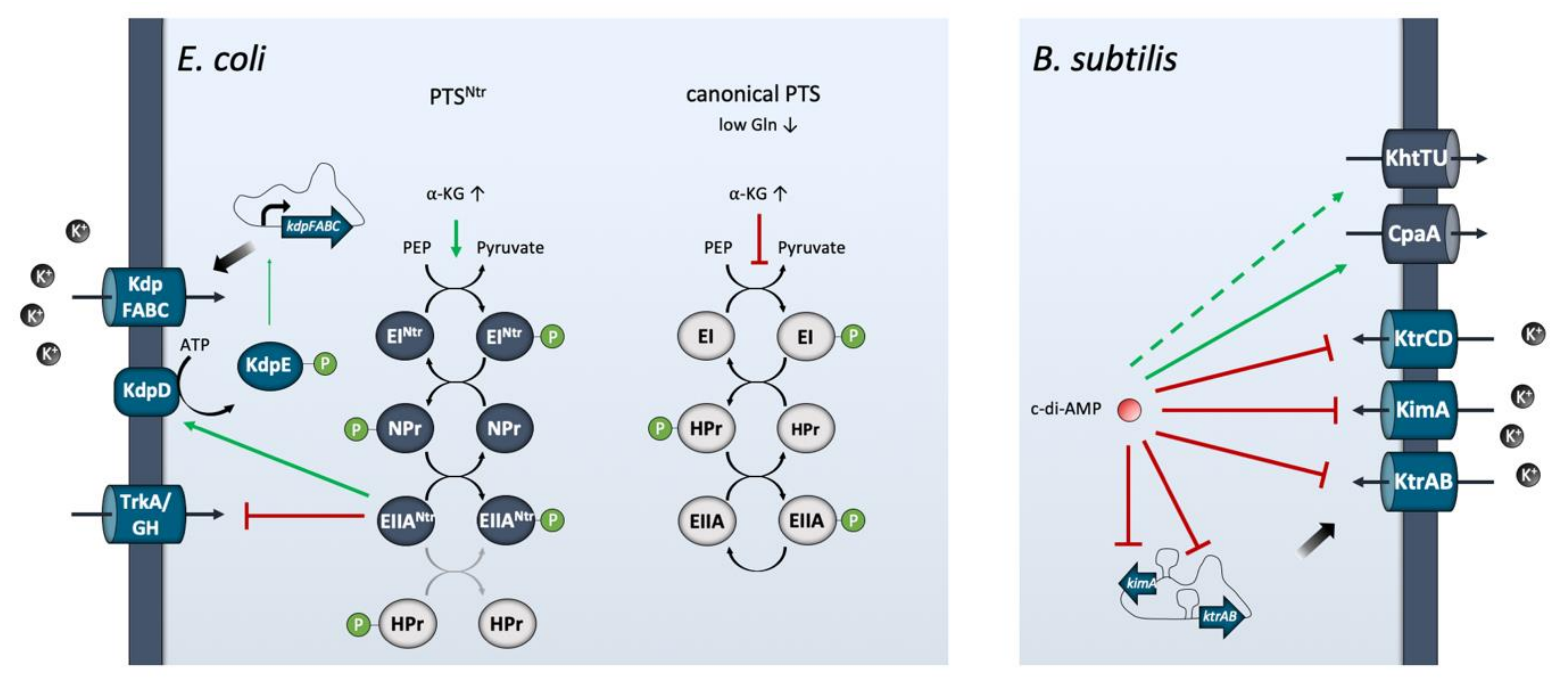

Fig. 7.1. The PTS ${ }^{\text {Ntr }}$ and c-di-AMP signal transduction pathways are analogous in their function as regulators of potassium homeostasis. Left: The control of potassium homeostasis in E. coli is mediated by the PTS ${ }^{\mathrm{Ntr}}$ system. Dephoshorylated ElIA ${ }^{\mathrm{Ntr}}$ inhibits the low-affinity potassium channel TrkA (Lee et al., 2007), and activates the twocomponent system KdpDE which leads to the expression of the high-affinity potassium transporter KdpFABC (Lüttmann et al., 2009). Right: The control of potassium homeostasis by the nucleotide second messenger c-di$A M P$. The nucleotide binds to the 5'UTR preceding the genes encoding the high-affinity potassium transporters KtrAB and KimA. c-di-AMP also binds and inhibits the potassium importer KtrAB, KimA, and KtrCD, and the potassium exporters KhtTU and CpaA (see Chapter 3; Gundlach et al., 2017b; Rocha et al., 2019). Activation of CpaA by c-di-AMP has been shown in S. aureus (Chin et al., 2015). Type of regulation: red, inhibition; green, activation; green dashed line, supposed activation. Abbreviations: $\alpha-K G, \alpha$-ketoglutarate; Gln, Glutamine; PEP, Phosphoenolpyruvat; c-di-AMP, cyclic di-AMP.

\subsection{New c-di-AMP functions and targets}

The number of identified c-di-AMP targets rapidly increased after the nucleotide second messenger has been discovered more than a decade ago in the crystal structure of the synthesizing enzyme DisA (Witte et al., 2008). A known motif for c-di-AMP binding is the RCK_C domain (Corrigan et al., 2013). These domains regulate the gating of potassium ions through both bacterial and eukaryotic membranes (Jiang et al., 2001). A second structurally conserved domain that is able to bind the nucleotide is the CBS domain (Sureka et al., 2014; Schuster et al., 2016; Huynh et al., 2016; Devaux et al., 2018b). In this study we have demonstrated that four out of five RCK_C domain containing proteins (KtrA, KtrC, KhtT, YjbO/CpaA), three out of 16 CBS domain containing proteins (MgtE, DarB, OpuCA), 
and KimA from B. subtilis bind the second messenger (see Chapter 3; Gundlach et al., 2019). Thus, not only all three potassium uptake systems seem to be controlled by c-di-AMP, but further identification of the potassium exporters KhtT and CpaA as c-di-AMP receptors reveals the global effect of the nucleotide on potassium homeostasis (Fig.7.2). KhtT and CpaA are also present in S. aureus and binding of c-di-AMP to CpaA leads to stimulation of potassium export (Corrigan et al., 2013; Chin et al., 2015). In addition, binding of the nucleotide to the CBS-domain-containing magnesium transporter MgtE indicates that c-di-AMP is involved in regulation of the transport of the most abundant divalent cation as well (see Chapter 3; Gundlach et al., 2019). Similar to kimA and ktrAB, mgtE expression is controlled by a riboswitch and a role of c-di-AMP in transcriptional control of the main magnesium transporter of B. subtilis seems plausible. However, in contrast to the riboswitch preceding the kimA and $k \operatorname{tr} A B$ loci, the $m g t E$ riboswitch has so far only been observed to respond to Mg ions (Dann et al., 2007; Ramesh and Winkler, 2010). c-di-AMP plays a role in the regulation of compatible solute uptake systems and proteins of the Opu family were described as receptor proteins in other species before (Schuster et al., 2016; Huynh et al., 2016; Devaux et al., 2018b). The identification of OpuCA as a c-di-AMP target in B. subtilis is not surprising and only confirms the strong conservation of this regulation (see Chapter 3; Gundlach et al., 2019). The third novel target from the CBS-domain family in B. subtilis is DarB. Like DarA, DarB does not have a secondary function next to acting as a receptor. For this reason, these two proteins are classified as signal transduction proteins. Identification of c-di-AMP receptor proteins from both bacterial and eukaryotic organisms relied so far on protein pulldowns via c-di-AMP-linked sepharose or magnetic beads, and the DRaCALA assays (see Chapter 3; Corrigan et al., 2013; Sureka et al., 2014; Gundlach et al., 2015a; Kampf et al., 2017; McFarland et al., 2017; Blötz et al., 2017; Gundlach et al., 2019). Protein pulldowns proved to be the method of choice, when the global screen for targets was the goal. This initial idea was often confirmed by methods analyzing the binding of the protein to radiolabeled [ ${ }^{32}$ P]c-di-AMP, like the DRaCALA assay (Corrigan et al., 2013). In addition, this method was used to test binding of the nucleotide to a selection of specific proteins, e.g. those containing previously identified c-di-AMP binding domains (see Chapter 3; Gundlach et al., 2019). However, this direct testing of candidates requires an initial hypothesis and could hinder us from identifying targets outside of the known cluster. Although the pulldown experiments via the c-di-AMP-coupled beads led to the identification of several targets, the identified proteins were mainly cytosolic proteins. Such pulldowns with $B$. subtilis crude extracts for instance led to the identification of the signal transduction protein DarA, while this experiment gave no hint to additional receptors (Gundlach et al., 2015a). However, this study revealed the existence of more targets in B. subtilis (see Chapter 3; Gundlach et al., 2019). Since DarA and DarB bind the nucleotide with a similar affinity (see Chapter 6; Gundlach et al., 2015a)., it remains unclear why DarB was not identified in the initial pulldown. This reveals another 
weakness of this type of experiment, which is that non-constitutive or poorly expressed proteins can be missed. B. subtilis encodes the sporulation-specific diadenylate cyclase CdaS, allowing the speculation that c-di-AMP could play some role during sporulation or germination. Many genes are sporulation-specific and are not expressed under vegetative growth conditions, which are often used for this type of experiments. Accordingly the respective proteins are missed in the standard experimental set-up. In order to shed light on the whole complexity of c-di-AMP regulation, the abovementioned difficulties should be taken into account when looking for c-di-AMP target proteins in the future.

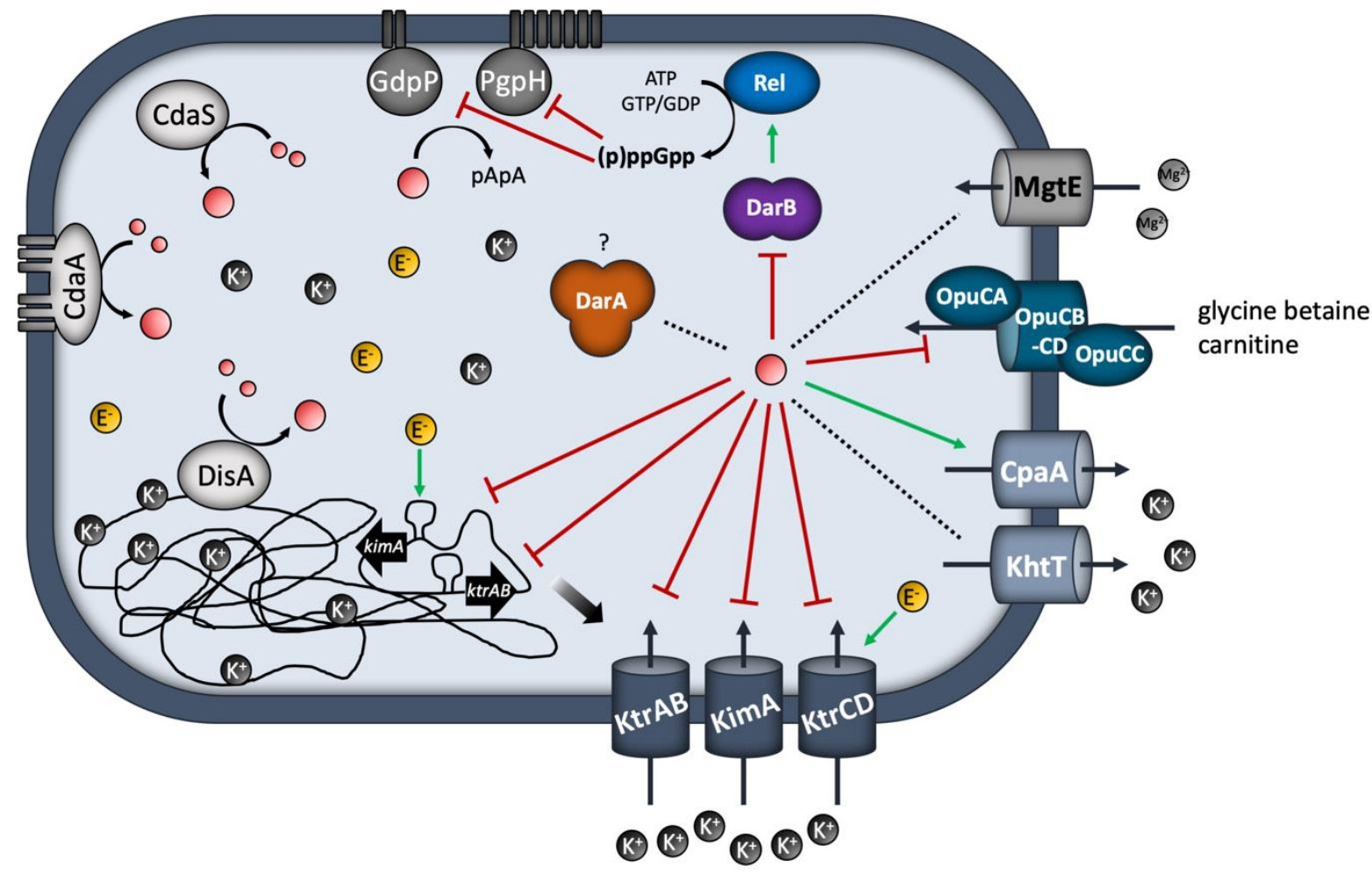

Fig. 7.2 c-di-AMP signaling in B. subtilis. B. subtilis encodes three diadenylate cyclases, CdaA, DisA, and CdaS and two phosphodiesterases, PgpH and GdpP. The c-di-AMP molecule (big red ball) controls ion transport and osmoregulation by inhibiting the $y d a O$ riboswitch, the potassium channels $K \operatorname{trAB}$ and $K \operatorname{trCD}$, the potassium transporter KimA, and the osmolyte uptake system Opu. The nucleotide binds the potassium exporter KhtT and CpaA. The second messenger binds the two signal transduction proteins DarA and DarB. Apo-DarB functions as an activator for Rel-dependent (p)ppGpp synthesis. Type of regulation: red, inhibition; green, activation; green dashed line, supposed activation black dashed line, unknown.

The creation of a c-di-AMP-depletion strain has been struggling and was only possible under certain conditions, that were low amounts of potassium and ammonium as a nitrogen source (Gundlach et al., 2017b). The toxicity of potassium has been analyzed before and could be bypassed by a hyperactive NhaK mutant protein that prevents toxic accumulation of potassium ions by increased export 
(Gundlach et al., 2017b). In this study, we were interested in how the $\Delta$ dac strain responds to the presence of glutamate, or even cultivation on complex medium (see Chapter 5). To do so, we isolated several suppressor mutants from minimal medium containing glutamate as a nitrogen source and low amounts of potassium. We observed that the adaptation to glutamate seems to be more complex than to potassium. In fact, the suppressors contained pairs of mutations that were affecting glutamate and potassium homeostasis, as well as phospholipid biosynthesis, flagellar, and respiration related genes. Many suppressors acquired loss-of-function mutations in AimA that we identified and characterized as the main low-affinity glutamate transporter of $B$. subtilis. The direct toxicity of glutamate is underlined by the decryptification of the second glutamate dehydrogenase of $B$. subtilis, GudB, as well as by a very specific mutation in the gate of the mechanosensitive channel $\mathrm{YfkC}$, that we suppose changes the selectivity and allows glutamate export. To our surprise, many suppressors contained mutations that were related to the uncontrolled potassium uptake of a $\Delta d a c$ strain. That was unexpected as the selection for suppressors happened with very low potassium in the medium. However, this can be explained by the increase in affinity of KtrCD for potassium in the presence of glutamate (see Chapter 4). That seven out of 15 suppressor mutants had mutations in essential genes involved in phospholipid biosynthesis, suggests that this is either a way to respond to the osmotic stress that is induced by the unbalanced potassium and glutamate homeostasis, or that this pathway might be somehow misregulated without c-di-AMP. Most genes involved in phospholipid synthesis are essential. Moreover, the ACC complex, as well as $\mathrm{PIsC}$, which were targets for mutations in our suppressor screen, display the major checkpoints for regulation of this pathway. The ACC complex generates malonyl-CoA and this central intermediate of fatty acid biosynthesis serves as the inducer for the transcriptional repressor FapR resulting in expression of the FapR regulon including PIsC (Schujman et al., 2003). Accumulation of malonyl-CoA in the $\Delta d a c$ strain would explain both the mutations in acc and $p l s C$ as they would prevent further synthesis of the intermediate and phosphatic acid, the central precursor for any phospholipid. That the suppressors acquired mutations in these essential genes, and more importantly in highly conserved regions of the proteins, suggests that the mutant proteins are very likely reduced in their biosynthetic activity. However, future research should focus on the exact molecular mechanism underlying this observation as it can not be excluded that the whole pathway is deregulated itself. As some of the genes involved in this pathway are membrane-associated, these proteins could have been missed in the previous search for c-di-AMP targets.

The isolation of suppressor mutants from complex medium was more challenging and turned out to require the additional deletion of either darA or darB as well as a two-step isolation procedure, where the first round of suppressor isolation happened under anaerobic conditions, and the second under aerobic conditions (see Chapter 5, Fig. 5.2 for the detailed isolation). The main problem under 
anaerobic conditions turned out to be the potassium/osmostress as all isolated suppressors acquired loss-of-function mutations in $\mathrm{KtrC}$ or OpuAB. That the isolation of suppressor mutants was only possible under anaerobic conditions where respiratory processes are downregulated, is in agreement with a study in S. aureus where suppressor isolation on complex medium was also only possible under anaerobic conditions (Zeden et al., 2018). This is supported by the loss-of-function mutations in ndhF in the glutamate screen and the transcriptomic analysis that showed that $n d h F$ is even 7-fold upregulated. This indicates that respiration is problematic without c-di-AMP. The Ktr transporters are driven by the proton motive force and KimA is a proton/potassium symporter (Diskowski et al., 2015; Tascón et al., 2020). Respiration might be toxic because it generates the proton motive force that would further fuel potassium uptake. This idea is supported by the fact that the aerobic suppressors of this two-step screen carried mutations in a second potassium transporter (KimA or KtrB, see Fig. 5.2). This indicates that the potassium stress for a $\Delta d a c$ mutant is indeed bigger under aerobic conditions where respiratory processes are used to produce chemical energy. However, potassium stress was not the only problem, as the suppressors acquired additional mutations in $\operatorname{dar} A, \operatorname{cod} Y$, and ссрA. The occurrence of loss-of-funtion mutations in darA were discussed above (see 7.2), and the mutations in $\operatorname{cod} Y$ and $c c p A$ indicate that there might be some metabolic problems, that are caused by the depletion of c-di-AMP. Getting to the bottom of this would answer some central questions about the potential impact of the nucleotide on carbon and nitrogen metabolism.

\section{4 c-di-AMP and (p)ppGpp crosstalk is mediated by DarB}

In this work, the c-di-AMP receptor DarB was identified and characterized as an activator of Reldependent (p)ppGpp synthesis (see Chapter 6; Fig. 7.2). Under conditions that induce low c-di-AMP levels, apo-DarB binds Rel. This likely induces a conformational change which leads to activation of the synthetase activity of Rel and induction of the stringent response. Upon induction of the stringent response, cells experience a global reorganization on both, expression and protein level. In B. subtilis, the regulon of the stringent response consists of 44 operons, 40 of them under negative regulation, and four under positive (Zhu and Stülke, 2018). This stress response is triggered by the accumulation of the nucleotide second messenger ( $\mathrm{p}$ )ppGpp. The so-called alarmone is synthesized and degraded by homologs of the RSH family (RSH, RelA-SppoT Homologs). RSHs can be divided into long bifunctional RSH, like Rel and SpoT, long monofunctional RSH, like RelA, short synthetases (SAS), and short hydrolases (SAH). In addition to a long bifunctional RSH enzyme, some bacteria also encode a long monofunctional RSH enzyme. A prominent example for this is E. coli, which encodes for both the bifunctional SpoT, as well as for the long monofunctional RelA enzyme. RelA of $E$. coli shares the same domain architecture as its bifunctional RSH counterpart, however, the hydrolase domain of RelA is 
degenerated and inactive (Aravind and Koonin, 1998). Short RSH enzymes are exclusively conserved among Gram-positive bacteria and might allow fine-tuning of intracellular ( $p$ )ppGpp levels. In contrast to SAH enzymes that are only present in a few bacteria, as for example L. monocytogenes, SAS enzymes are more wide-spread. The genome of some bacteria, like those of B. subtilis and S. aureus, encode even two SAS type enzymes. This already suggests, that the fine-tuning of the stringent response might be of special importance in these organisms. The two enzymatic activities of Rel/SpoT homologs are mutually exclusive (Mechold et al., 2002; Hogg et al., 2004). If the cell experiences amino acid starvation, Rel binds uncharged tRNAs via the TGS-domain. This directly inhibits the dimerization of Rel and allows association to the ribosome. This induces a structural rearrangement, that includes dissociation of the TGS-domain from the synthetase domain, and activation of ( $p$ )ppGpp synthesis (Pausch et al., 2020). Under non-stress conditions, Rel is known to be an active hydrolase. How Rel is kept in this state, and how the opposing enzymatic activities of the enzyme are coordinated, has only recently been described (Pausch et al., 2020). Under relaxed conditions, the synthetase activity of Rel is inhibited by association of the TGS domain, a subdomain of the C-terminal region of Rel, with the synthetase domain (Pausch et al., 2020). Rel forms homodimers, when not associated to the ribosome (Yang and Ishiguro, 2001). The role of oligomerization on the opposing enzymatic activities of Rel has been discussed (Gropp et al., 2001; Yang and Ishiguro, 2001; Avarbock et al., 2005), however, Pausch et al. show that the oligomerization status of Rel does not affect its enzymatic activities, but rather interferes with the interaction with uncharged tRNAs.

The presence of uncharged tRNAs as an indication for amino acid starvation might be the main factor inducing the stringent response (Cashel and Gallant, 1969; Hauryliuk et al., 2015). However, the induced global cellular reprogramming responds to several stresses other than amino acid limitation. In order to activate this response, bacteria have developed various mechanisms that allow the switch of Rel's enzymatic activity. A stress situation that impacts the cell similarly to amino acid starvation, is nitrogen deprivation. The influence of nitrogen deprivation on the stringent response has been observed in several organisms, however, the way of regulation seems to differ completely. Transcription of the relA gene in E.coli for example, is regulated by the nitrogen-responsive transcriptional regulator NtrC (Brown et al., 2014). When intracellular glutamine level drop, NtrC becomes phosphorylated and activates the transcription of hundreds of genes, among them the monofunctional relA gene (Brown et al., 2014). Induction of the stringent response upon glutamine deprivation has also been observed in C. crescentus, Sinorhizobium meliloti, and R. eutropha where EllA ${ }^{\text {NtrA }}$ directly affects (p)ppGpp synthesis by interaction with the ACT domain of SpoT/Rel (Karstens et al., 2014; Ronneau et al., 2016; Ronneau et al., 2019a). While phosphorylated EIIA ${ }^{\text {NtrA }}$ stimulates Reldependent (p)ppGpp synthesis in C. crescentus and S. meliloti (Goodwin and Gage, 2014; Ronneau et 
al., 2016), only non-phosphorylated EIIA ${ }^{\mathrm{NtrA}}$ has been observed to interact with SpoT in R. eutropha (Karstens et al., 2014). The second bifunctional RSH enzyme from E. coli, SpoT, is regulated upon several different stresses other than amino acid limitation. The enzyme responds to fatty acid limitation via direct interaction with the acyl carrier protein (ACP) (Battesti and Bouveret, 2006). ACP can exist as free apo-ACP, and as ACP bound to intermediates of the fatty acid synthesis pathway. The latter form of ACP is able to interact with the TGS domain of SpoT and induce the synthesis of (p)ppGpp. Hence, ACP seems to serve as a sensor for the fatty acid pool of the cell by sensing accumulating intermediates of the fatty acid biosynthesis. Unlike RelA, SpoT is a bifunctional enzyme, so it appears reasonable, that both enzymatic activities are subject to regulation. In $E$. coli the enzyme is regulated by the Rsd protein that binds to SpoT to stimulate its hydrolytic activity during carbon downshift (Lee et al., 2018). Under carbon limitation, HPr is phosphorylated and cannot bind the Rsd protein (Park et al., 2013). Hence, Rsd is free and able to bind the TGS domain of SpoT. Rsd keeps the enzyme in the hydrolase-on state, to prevent intoxication by excessive (p)ppGpp synthesized by stimulated RelA caused by poor carbon sources (Lazzarini and Cashel 1971; Lee et al., 2018). This ensures induction of the stringent response on one hand, and on the other hand prevents the cell from uncontrolled (p)ppGpp accumulation. The hydrolytic activity of SpoT/Rel proteins in organisms that encode only one long RSH enzyme, is most likely not subject to positive regulation (e.g. stimulation) as it is the case for E. coli, which encodes for a second active synthase. This regulatory feature is important when bacteria have to ensure that they never, under any condition, accumulate ( $p$ )ppGpp to toxic levels. By regulating the hydrolase domain of SpoT, they create sort of a safety valve that prevents intoxication.

The regulation of RSH enzymes by interaction of protein or RNA regulators with regions of the C terminal domain, is a common mechanism. The C-terminal region of RSH enzymes is crucial for the oligomerization and deletion of this region is known to abolish dimerization (see Chapter 6; Gropp et al., 2001; Yang and Ishiguro, 2001). However, to date there are also a few examples described, where the enzymatic activity of RSH enzmyes is directly controlled by interaction of the regulator with the Nterminal region of the protein that harbors the enzymatically active domains. In E. coli, the switch of SpoT from being an active hydrolase to becoming an active synthetase is mediated by interaction of YtfK with both the hydrolase and the synthetase domain (Germain et al., 2019). YtfK is involved in the induction of the stringent response upon fatty acid and phosphate starvation (Germain et al., 2019), however, the exact mechanisms remain unclear. Since YtfK is not conserved outside of the $\gamma$-proteobacteria, this regulatory mechanism seems to be limited to only a handful of bacteria. As Rsd, the GTPase CgtA stimulates the hydrolase activity of SpoT in E. coli (Wout et al., 2004). CgtA is strongly associated to the ribosome under nutrient starvation conditions, however, when nutrients become 
available again, the association is loosened, and CgtA is thought to be a crucial factor keeping SpoT as an active hydrolase (Wout et al., 2004; Raskin et al., 2007). CgtA binds SpoT via both, the C-terminal and $\mathrm{N}$-terminal domain, and deletion of one of the domains was not able to abolish the interaction (Wout et al., 2004). The stimulation of Rel's synthetase activity by the c-di-AMP receptor DarB, as shown in this work, is to date one of the few examples for regulation of Rel activity via direct interaction with the enzyme's active domains. This type of regulation appears to be the exception rather than the rule. The reasons for this are not clear. Since the conformation of the synthetase and hydrolase domain, that allows for the switch in the enzymatic activity of RSH enzymes, is very specific, it could have been hard to integrate an additional effector in this process during evolution. Similarly, the two active centers are arranged close to each other, making it hard to regulate one without affecting the other. Since accumulation of (p)ppGpp is toxic, the hydrolytic activity of the enzyme should immediately be available. Hence, it is most likely that RSH enzymes are controlled via the C-terminal region, because this type of regulation has proven to be most compatible with the conformation of the enzyme. Yet, DarB is an excellent example that it is still possible to regulate RSH enzymes by binding to the enzymatic active sites.

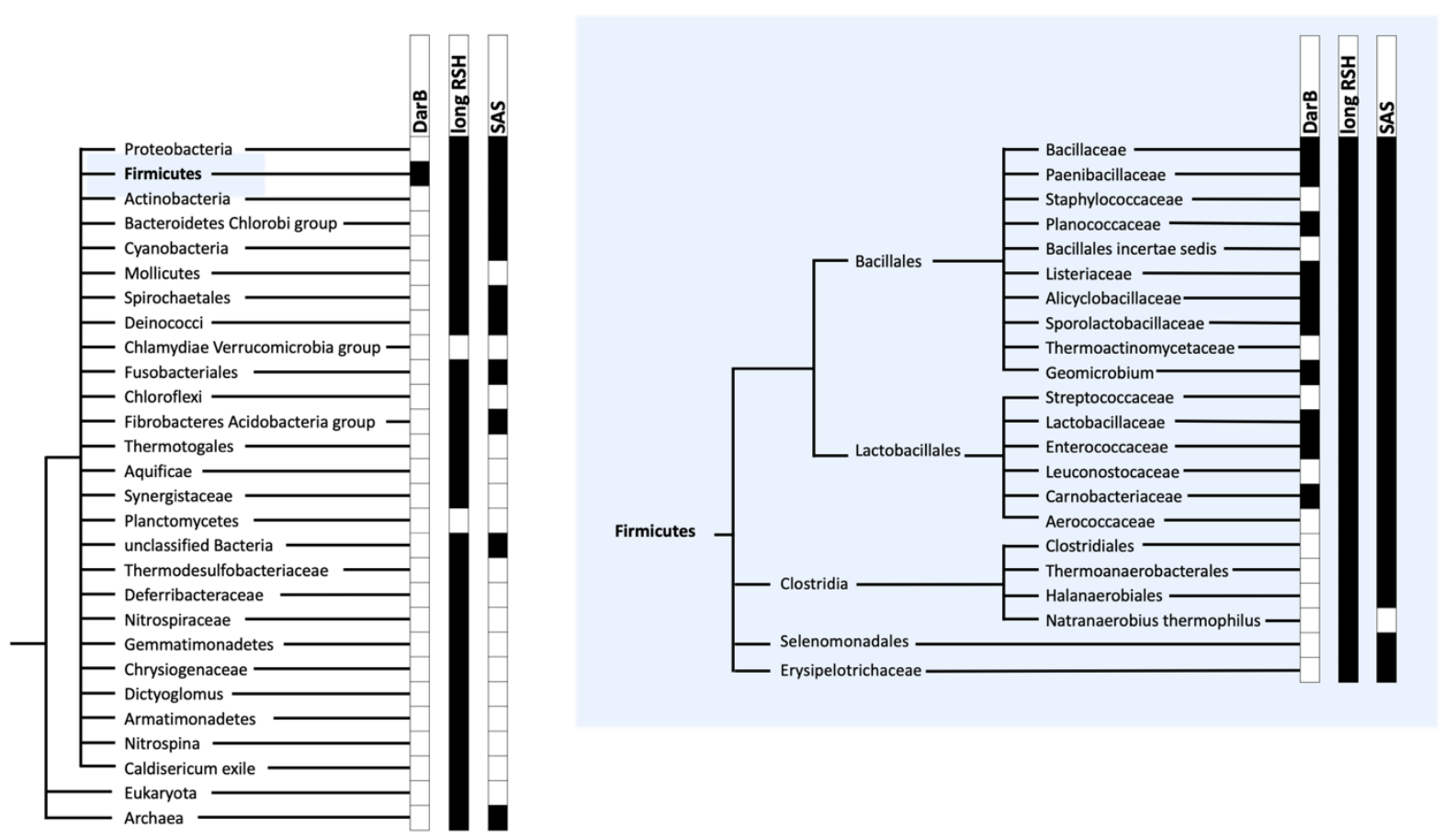

Fig. 7.3. Cooccurrence of the c-di-AMP receptor DarB, long RSH enzymes, and SAS enzymes and their taxonomic distribution. The distribution within the respective group is depicted by black color. The data derived from STRING and Atkinson et al., 2011. 
Moreover, DarB is a c-di-AMP binding protein and this second messenger is the life-sustaining answer to changing environmental potassium levels. In this work, we were able to connect this signaling process to the stringent response through stimulation of Rel's synthetase activity by the c-diAMP receptor DarB. This newly identified event of nucleotide second messenger crosstalk shows that different second messenger signaling pathways evolved in a way from responding to a specific number of signals to inducing different cellular responses via crosstalk with other signaling pathways. Crosstalk fine-tunes a global cellular response to certain stresses that otherwise might be detrimental for growth and survival of the bacteria, if not tightly regulated.

In order to put the relevance of the interaction between the c-di-AMP binding protein DarB and the dual function (p)ppGpp synthetase/hydrolase Rel into context, the co-occurrence of these two genes is of particular interest. RSH enzymes are conserved in all kingdoms of life (Fig. 7.3; Cellini et al., 2004; Atkinson et al., 2011), making the stringent response an universal stress response. c-di-AMP signaling on the other hand is a solely bacterial and archaeal stress response. In contrast to (p)ppGpp, which is synthesized and degraded from one class of enzymes, c-di-AMP can be can be degraded by several enzmyes (Table 2.1). Thus, the set of c-di-AMP related genes differs a lot between organisms and the conservation of c-di-AMP signaling can not be explained with the conservation of one enzyme, as it is the case for ( $p$ )ppGpp signaling. This already suggests, that ( $p$ )ppGpp signaling might have evolved fairly early during evolution and that an early gene duplication event led to the presence of additonal RSH enzymes in one genome (Atkinson et al., 2011). In contrast to (p)ppGpp signaling, c-di-AMP signaling has likely evolved several times independently. Accordingly, the response to the same, c-di-AMP-responsive stress can differ remarkably between different organisms. The increased attention for nucleotide second messengers in the past few years as well as the establishment of new methods that allow fast and easy screening for novel receptors, led to a constant increase in the number of novel c-di-AMP targets (see 7.3 for details). The DarB protein consists of two CBS-domains and forms very stable homodimers. The two monomers are coordinated in a parallel, head-to-head orientation and one DarB dimer was shown to bind two molecules of c-di-AMP (Heidemann, 2020). darB is conserved along with c-di-AMP synthesizing and degrading enzymes, making it reasonable that being part of the c-di-AMP signaling cascade is the major function of the protein. The protein is, with a few exceptions, limited to the class of Bacilli (Fig 7.3). Here it can be found in almost every family, with the exception of Staphylococcaceae, Bacillales incertae sedis, and Leuconostocaceae (Fig. 7.3). As both, c-di-AMP and (p)ppGpp signaling are present in these organisms, the specific stress response mediated by DarB in Bacilli, might be fulfilled by a different protein in these organisms. In this work it was shown that c-di-AMP and (p)ppGpp signaling are used by the cell to respond and adapt to otherwise toxic potassium limitations. The high affinity of the receptor for its ligand is a prerequisite 
to allow fast and efficient signal transmission. The affinity of DarB for its effector Rel $(0.65 \mu \mathrm{M})$ is in the same molar range in which the enzyme is present in the cell $(0.6 \mu \mathrm{M})$. The extremely high affinity of DarB for c-di-AMP ( $45 \mathrm{nM}$ ) on the other hand ensures that the tiniest amounts of the nucleotide are sufficient to stop the global stress response induced by activation of the stringent response. Since the stringent response induces a global metabolic reprogramming, it is of major importance that both the induction but also the shutdown are fast and efficient. Even though the exact mechanism by which DarB acts as an activator of the stringent response might not be very conserved among bacteria outside of the phylum of Firmicutes, the general principle can still be. In these organisms the response to potassium limitation could be mediated by a different protein, potentially even a CBS-domain containing protein. Since CBS-domains differ sequence-wise remarkably, even within the same organism, the prediction of potential CBS-domain containing proteins that might fulfil a similar function to DarB, is challenging. The comparison of the CBS1 domain of DarB with the CBS1 domain of the transcriptional regulator $\mathrm{CcpN}$ and the magnesium transporter $\mathrm{MgtE}$, all sequences from B. subtilis, demonstrates the low sequence conservation of these domains. The three CBS-domains share only $14-21 \%$ sequence identity and $28-43 \%$ sequence similarity, which is rather low given the fact that they are encoded in the same genome. In contrast, the structural comparison confirms that the likelihood that the three proteins share a very similar fold, is extremely high (TMscore $>0.74$, comparison with RaptorX, Structure Alignment tool). If the structural comparison of two protein structures reveals a TMscore $>0.6$, the chance that these two proteins share a similar fold is considered as very likely (>90\%) (Xu and Zhang, 2010). This shows, that even though the CBS-domain containing proteins might differ significantly in their sequence, their structural fold, and accordingly their cellular function, might be conserved with a much higher probability. Although DarB itself (sequence based) is not very conserved, the fold of the protein and hence its function might still be. For this reason, it is of major interest to look for potential functional homologs of DarB in organisms that do not encode a DarB homolog. One organism that does not encode a DarB homolog, but that uses both c-di-AMP and (p)ppGpp signaling, is $S$. aureus. The crosstalk between these two signaling pathways has been described before (Corrigan et al., 2015), however, the similar phenotypes of $\Delta$ dac strains raises the question, whether S. aureus responds to potassium limitation in a similar way as B. subtilis, and consequently, if there is a protein analogous to DarB. The genome of $S$. aureus encodes for six other CBS-domain containing proteins. Among them the IMP-dehydrogenase GuaB, the magnesium importer MgtE, the magnesium exporter MpfA and the potential magnesium exporter YhdT, and the regulators $\mathrm{CcpN}$ and Ytol. While binding of c-di-AMP was observed for MgtE in B. subtilis (see Chapter 3; Gundlach et al., 2019), the S. aureus homolog was so far not identified as a c-di-AMP binder. Binding of c-di-AMP to one of the five remaining CBS-domain proteins, has so far neither been 
observed in S. aureus (Schuster et al., 2016), nor in B. subtilis (see Chapter 3; Gundlach et al., 2019). However, to fulfil a function analogous to DarB, the potential functional homolog must at least fulfil the following criterion: it has to bind c-di-AMP. Since the genome of S. aureus apparently does not encode for such a protein, at least none harbouring a CBS-domain, it remains speculative for the moment if $S$. aureus is able to respond to potassium limitation in a similar manner as $B$. subtilis.

The connection between c-di-AMP and (p)ppGpp metabolism is not completely relying on the presence of DarB, as the crosstalk of the two second messenger has been described on a second level. The inhibitory effect of (p)ppGpp has so far been observed for PgpH-like (HD) and GdpP-like (DHH/DHHA1) c-di-AMP degrading phosphodiesterases in B. subtilis, L. monocytogenes, and also in S. aureus (Rao et al., 2010; Huynh et al., 2015; Corrigan et al., 2015; Bowman et al., 2016). As the enzymatic reaction leading to the cleavage of the phosphodiester bond is distinct in the two types of enzymes, it appears likely that the inhibition by ( $p$ )ppGpp has evolved at least twice independently. This demonstrates the importance of the regulatory circuit between the c-di-AMP and (p)ppGpp signaling pathways. Simultaneously, the question arises whether this crosstalk also exists in organisms lacking both DarB and a phosphodiesterase of either the PgpH- or the GdpP-type, as it appears to be the case for example for Actinobacteria and Mollicutes. The potential impact of (p)ppGpp on phosphodiesterases of the DhhP/NrnA, CdnP, and AtaC type should be the focus of future research. Before the regulatory importance of DarB in this nucleotide second messenger crosstalk has been elucidated in this work, and for its homolog CbpB in L. monocytogenes (Peterson et al., 2020), the described phenotypes of $\Delta d a c(\Delta d a c)$ or $\Delta p d e$ mutants were controversial. For example, deletion of the phosphodiesterase GdpP in S. aureus resulted in an increase in both c-di-AMP and (p)ppGpp levels (Corrigan et al., 2015), and deletion of the diadenylate cyclase DacA in L. monocytogenes caused an increase in (p)ppGpp levels as well (Whiteley et al., 2015). This was tried to be explained by the low intracellular concentration of c-di-AMP, where changes in abundancy in either direction could affect (p)ppGpp levels (Steinchen et al., 2020). These species-specific differences, that have been observed in the past, can now be explained by the presence of the additional, newly described, regulatory mechanism in both B. subtilis and L. monocytogenes. The observed increase in (p)ppGpp levels upon c-di-AMP depletion in L. monocytogenes is induced by Apo-CbpB, that was shown to act as an activator of Rel-dependent (p)ppGpp synthesis (Peterson et al., 2020). Since a DarB/CbpB homolog is missing in S. aureus, this increase in (p)ppGpp upon c-di-AMP depletion is most likely not visible in this organism. An increase of (p)ppGpp in $\Delta p d e$ mutants, however, might have different, so far unknown reasons.

c-di-AMP and ElIA ${ }^{\mathrm{Ntr}}$ are not only analogous in their function as major regulators in potassium homeostasis as discussed above, apparently they also share the ability to regulate the stringent response. Phosphorylated EIIA ${ }^{\text {Ntr }}$ binds the ACT domain of SpoT/Rel and stimulates (p)ppGpp synthesis 
in $\alpha$ - and $\beta$-proteobacteria (Gonzalez and Collier, 2014; De Nisco et al., 2014; Karstens et al., 2014; Ronneau et al., 2019a). The coordinated regulation of potassium homeostasis and the stringent response might be a conserved mechanism, which appears to be executed to the same extent by either c-di-AMP or EllA ${ }^{\mathrm{Ntr}}$.

\subsection{Open questions}

Since the discovery of c-di-AMP twelve years ago (Witte et al., 2008), the role of the second messenger has been studied in many bacteria. c-di-AMP is the only known essential second messenger, and in the beginning it was thought that the essentiality is caused by the regulation of an essential target protein. However, none of the identified receptors is essential by itself. Despite species-specific differences, the involvement of the nucleotide in central homeostatic processes has been demonstrated for many organisms (Corrigan et al., 2013; Schuster et al., 2016; Huynh et al., 2016; Devaux et al., 2018b; Whiteley et al., 2017; Quintana et al., 2019; Gundlach et al., 2019; Gibhardt et al., 2019; Zarrella et al., 2018; Bai et al., 2013; Chin et al., 2015; Moscoso et al., 2016; Zeden et al., 2018). This conserved link between c-di-AMP and central ion transport processes might explain why it is, in contrast to e.g. the life-style modulator c-di-GMP, essential for bacteria. Although more and more receptor proteins are discovered and the role of c-di-AMP for cellular physiology becomes clearer, however, only little is known about the signals affecting the homeostasis of the second messenger itself. c-di-AMP levels are affected by potassium and glutamate (Gundlach et al., 2015b; Gundlach et al., 2017b; Zarrella et al., 2018), but if this is the result of transcriptional control or if the diadenylate cyclases and phosphodiesterases are controlled on protein level, or maybe also both, are important questions to be answered in the future. Moreover, it will be of special interest to investigate the crosstalk between c-di-AMP and (p)ppGpp signaling deeper, to find out if the stringent response in B. subtilis could also be induced upon glutamine limitation for instance, as this signal is known to be integrated via the PTS ${ }^{\mathrm{Ntr}}$ system in $\alpha$-proteobacteria (Ronneau et al., 2019). Revealing the function of the second signal transduction protein DarA will shed light on additional stress signals that are sensed by the second messenger and that might contribute to its essentiality. 


\section{Chapter 8 - References}

Abdelhamid, Y., Brear, P., Greenhalgh, J., Chee, X., Rahman, T., et al. (2019) Evolutionary plasticity in the allosteric regulator-binding site of pyruvate kinase isoform PykA from Pseudomonas aeruginosa. J Biol Chem 294: 15505-15516.

Aguado-Llera, D., Oyenarte, I., Martínez-Cruz, L.A., and Neira, J.L. (2010) The CBS domain protein MJ0729 of Methanocaldococcus jannaschii binds DNA. FEBS Lett 584: 4485-4489.

Albright, R.A., Ibar, J.L.V., Kim, C.U., Gruner, S.M., and Morais-Cabral, J.H. (2006) The RCK Domain of the KtrAB K ${ }^{+}$Transporter: Multiple Conformations of an Octameric Ring. Cell 126: 1147-1159.

Almblad, H., Harrison, J.J., Rybtke, M., Groizeleau, J., Givskov, M., et al. (2015) The cyclic AMP-Vfr signaling pathway in Pseudomonas aeruginosa is inhibited by cyclic Di-GMP. J Bacteriol 197: 2190-2200.

Altenbuchner, J. (2016) Editing of the Bacillus subtilis Genome by the CRISPR-Cas9 System. Appl Environ Microbiol 82: 5421-5427.

Anantharaman, V., and Aravind, L. (2003) Application of comparative genomics in the identification and analysis of novel families of membrane-associated receptors in bacteria. BMC Genomics 4: 34.

Andrade, W.A., Firon, A., Schmidt, T., Hornung, V., Fitzgerald, K.A., et al. (2016) Group B Streptococcus Degrades Cyclic-di-AMP to Modulate STING-Dependent Type I Interferon Production. Cell Host Microbe 20: 49-59.

Aravind, L., and Koonin, E. V. (1998) The HD domain defines a new superfamily of metal-dependent phosphohydrolases. Trends Biochem Sci 23: 469-472.

Atkinson, G.C., Tenson, T., and Hauryliuk, V. (2011) The RelA/SpoT Homolog (RSH) Superfamily: Distribution and Functional Evolution of ppGpp Synthetases and Hydrolases across the Tree of Life. PLoS One 6: e23479.

Auffinger, P., D'Ascenzo, L., and Ennifar, E. (2016) Sodium and Potassium Interactions with Nucleic Acids. In Metal lons in Life Sciences. pp. 167-201.

Avarbock, A., Avarbock, D., Teh, J.S., Buckstein, M., Wang, Z.M., et al. (2005) Functional regulation of the opposing ( $\mathrm{p}$ )ppGpp synthetase/hydrolase activities of RelMtb from Mycobacterium tuberculosis. Biochemistry 44: 9913-9923.

Bai, Y., Yang, J., Eisele, L.E., Underwood, A.J., Koestler, B.J., et al. (2013) Two DHH subfamily 1 proteins in Streptococcus pneumoniae possess cyclic Di-AMP phosphodiesterase activity and affect bacterial growth and virulence. J Bacteriol 195: 5123-5132.

Bai, Y., Yang, J., Zarrella, T.M., Zhang, Y., Metzger, D.W., et al. (2014) Cyclic Di-AMP Impairs Potassium 
Uptake Mediated by a Cyclic Di-AMP Binding Protein in Streptococcus pneumoniae. J Bacteriol 196: 614-623.

Barbe, V., Cruveiller, S., Kunst, F., Lenoble, P., Meurice, G., et al. (2009) From a consortium sequence to a unified sequence: The Bacillus subtilis 168 reference genome a decade later. Microbiology 155: 1758-1775.

Barker, J.R., Koestler, B.J., Carpenter, V.K., Burdette, D.L., Waters, C.M., et al. (2013) STING-Dependent Recognition of Cyclic di-AMP Mediates Type I Interferon Responses during Chlamydia trachomatis Infection. MBio 4: 1-11.

Barrick, J.E., Corbino, K.A., Winkler, W.C., Nahvi, A., Mandal, M., et al. (2004) New RNA motifs suggest an expanded scope for riboswitches in bacterial genetic control. Proc Natl Acad Sci U S A 101: 6421-6426.

Bateman, A. (1997) The structure of a domain common to archaebacteria and the homocystinuria disease protein. Trends Biochem Sci 22: 12-13.

Battesti, A., and Bouveret, E. (2006) Acyl carrier protein/SpoT interaction, the switch linking SpoTdependent stress response to fatty acid metabolism. Mol Microbiol 62: 1048-1063.

Baykov, A.A., Tuominen, H.K., and Lahti, R. (2011) The CBS Domain: A Protein Module with an Emerging Prominent Role in Regulation. ACS Chem Biol 6: 1156-1163.

Becker, M., Börngen, K., Nomura, T., Battle, A.R., Marin, K., et al. (2013) Glutamate efflux mediated by Corynebacterium glutamicum MscCG, Escherichia coli MscS, and their derivatives. Biochim Biophys Acta - Biomembr 1828: 1230-1240.

Bejerano-Sagie, M., Oppenheimer-Shaanan, Y., Berlatzky, I., Rouvinski, A., Meyerovich, M., et al. (2006) A Checkpoint Protein That Scans the Chromosome for Damage at the Start of Sporulation in Bacillus subtilis. Cell 125: 679-690.

Belitsky, B.R., and Sonenshein, A.L. (1998) Role and Regulation of Bacillus subtilis Glutamate Dehydrogenase Genes. J Bacteriol 180: 6298-6305.

Benda, M., Woelfel, S., Gunka, K., Klumpp, S., Poehlein, A., et al. (2020) Quasi-essentiality of RNase Y in Bacillus subtilis is caused by its critical role in the control of mRNA homeostasis. bioRxiv 2020.05.20.106237.

Bennett, B.D., Kimball, E.H., Gao, M., Osterhout, R., Dien, S.J. Van, et al. (2009) Absolute metabolite concentrations and implied enzyme active site occupancy in Escherichia coli. Nat Chem Biol 5: 593-599.

Bi, W., and Stambrook, P.J. (1998) Site-Directed Mutagenesis by Combined Chain Reaction. Anal Biochem 256: 137-140.

Bilder, P., Lightle, S., Bainbridge, G., Ohren, J., Finzel, B., et al. (2006) The Structure of the 
Carboxyltransferase Component of Acetyl-CoA Carboxylase Reveals a Zinc-Binding Motif Unique to the Bacterial Enzyme. Biochemistry 45: 1712-1722.

Blencke, H.-M. (2001) Genregulation des Zitronensäure-Zyklus in Bacillus subtilis als Spiegel seiner katabolen und anabolen Funktionen. Diplomarbeit.

Blencke, H.-M., Reif, I., Commichau, F.M., Detsch, C., Wacker, I., et al. (2006) Regulation of citB expression in Bacillus subtilis: integration of multiple metabolic signals in the citrate pool and by the general nitrogen regulatory system. Arch Microbiol 185: 136-146.

Block, K.F., Hammond, M.C., and Breaker, R.R. (2010) Evidence for widespread gene control function by the $y d a O$ riboswitch candidate. J Bacteriol 192: 3983-3989.

Blötz, C., Treffon, K., Kaever, V., Schwede, F., Hammer, E., et al. (2017) Identification of the Components Involved in Cyclic Di-AMP Signaling in Mycoplasma pneumoniae. Front Microbiol 8.

Booth, I.R., and Higgins, C.F. (1990) Enteric bacteria and osmotic stress: Intracellular potassium glutamate as a secondary signal of osmotic stress? FEMS Microbiol Lett 75: 239-246.

Bossemeyer, D., Schlosser, A., and Bakker, E.P. (1989) Specific cesium transport via the Escherichia coli Kup (TrkD) K+ uptake system. J Bacteriol 171: 2219-2221.

Bowman, L., Zeden, M.S., Schuster, C.F., Kaever, V., and Gründling, A. (2016) New Insights into the Cyclic Di-adenosine Monophosphate (c-di-AMP) Degradation Pathway and the Requirement of the Cyclic Dinucleotide for Acid Stress Resistance in Staphylococcus aureus. J Biol Chem 291: 26970-26986.

Bradford, M.M. (1976) A rapid and sensitive method for the quantitation of microgram quantities of protein utilizing the principle of protein-dye binding. Anal Biochem 72: 248-254.

Bramley, H.F., and Kornberg, H.L. (1987) Sequence homologies between proteins of bacterial phosphoenolpyruvate-dependent sugar phosphotransferase systems: identification of possible phosphate-carrying histidine residues. Proc Natl Acad Sci 84: 4777-4780.

Branda, S.S., Gonzalez-Pastor, J.E., Ben-Yehuda, S., Losick, R., and Kolter, R. (2001) Fruiting body formation by Bacillus subtilis. Proc Natl Acad Sci 98: 11621-11626.

Breaker, R.R. (2012) Riboswitches and the RNA World. Cold Spring Harb Perspect Biol 4: a003566a003566.

Bremer, E., and Krämer, R. (2019) Responses of Microorganisms to Osmotic Stress. Annu Rev Microbiol 73: 313-334.

Brill, J., Hoffmann, T., Bleisteiner, M., and Bremer, E. (2011) Osmotically Controlled Synthesis of the Compatible Solute Proline Is Critical for Cellular Defense of Bacillus subtilis against High Osmolarity. J Bacteriol 193: 5335-5346.

Brown, A., Fernández, I.S., Gordiyenko, Y., and Ramakrishnan, V. (2016) Ribosome-dependent 
activation of stringent control. Nature 534: 277-280.

Brown, D.R., Barton, G., Pan, Z., Buck, M., and Wigneshweraraj, S. (2014) Nitrogen stress response and stringent response are coupled in Escherichia coli. Nat Commun 5: 1-8.

Cadby, I.T., Basford, S.M., Nottingham, R., Meek, R., Lowry, R., et al. (2019) Nucleotide signaling pathway convergence in a CAMP-sensing bacterial c-di-GMP phosphodiesterase. EMBO J 38: 119.

Calvo, R.A., and Kearns, D.B. (2015) FlgM Is Secreted by the Flagellar Export Apparatus in Bacillus subtilis. J Bacteriol 197: 81-91.

Cashel, M., and Gallant, J. (1969) Two Compounds implicated in the Function of the RC Gene of Escherichia coli. Nature 221: 838-841.

Cellini, A., Scoarughi, G.L., Poggiali, P., Santino, I., Sessa, R., et al. (2004) Stringent control in the archaeal genus Sulfolobus. Res Microbiol 155: 98-104.

Chávez, S., Lucena, J.M., Reyes, J.C., Florencio, F.J., and Candau, P. (1999) The presence of glutamate dehydrogenase is a selective advantage for the cyanobacterium Synechocystis sp. strain PCC 6803 under nonexponential growth conditions. J Bacteriol 181: 808-813.

Chen, G.-Q., Cui, C., Mayer, M.L., and Gouaux, E. (1999) Functional characterization of a potassiumselective prokaryotic glutamate receptor. Nature 402: 817-821.

Cheng, Y., Korolev, N., and Nordenskiöld, L. (2006) Similarities and differences in interaction of $\mathrm{K}^{+}$and $\mathrm{Na}^{+}$with condensed ordered DNA. A molecular dynamics computer simulation study. Nucleic Acids Res 34: 686-696.

Chin, K.-H., Liang, J.-M., Yang, J.-G., Shih, M.-S., Tu, Z.-L., et al. (2015) Structural Insights into the Distinct Binding Mode of Cyclic Di-AMP with Sa CpaA_RCK. Biochemistry 54: 4936-4951.

Choi, P.H., Sureka, K., Woodward, J.J., and Tong, L. (2015) Molecular basis for the recognition of cyclicdi-AMP by PstA, a Pll-like signal transduction protein. Microbiology 361-374.

Choi, P.H., Vu, T.M.N., Pham, H.T., Woodward, J.J., Turner, M.S., et al. (2017) Structural and functional studies of pyruvate carboxylase regulation by cyclic di-AMP in lactic acid bacteria. Proc Natl Acad Sci U S A 114: E7226-E7235.

Collins, K.D. (1997) Charge Density-Dependent Strength. Biophys J 72: 65-76.

Commichau, F.M., Dickmanns, A., Gundlach, J., Ficner, R., and Stülke, J. (2015) A jack of all trades: the multiple roles of the unique essential second messenger cyclic di-AMP. Mol Microbiol 97: 189204.

Commichau, F.M., Gibhardt, J., Halbedel, S., Gundlach, J., and Stülke, J. (2018a) A Delicate Connection: c-di-AMP Affects Cell Integrity by Controlling Osmolyte Transport. Trends Microbiol 26: 175-185. Commichau, F.M., Gunka, K., Landmann, J.J., and Stülke, J. (2008) Glutamate metabolism in Bacillus 
subtilis: Gene expression and enzyme activities evolved to avoid futile cycles and to allow rapid responses to perturbations of the system. J Bacteriol .

Commichau, F.M., Heidemann, J.L., Ficner, R., and Stülke, J. (2018b) Making and Breaking of an Essential Poison: the Cyclases and Phosphodiesterases That Produce and Degrade the Essential Second Messenger Cyclic di-AMP in Bacteria. J Bacteriol 201.

Commichau, F.M., Pietack, N., and Stülke, J. (2013) Essential genes in Bacillus subtilis: a re-evaluation after ten years. Mol Biosyst 9: $1068 \mathrm{~h}$.

Corrigan, R.M., Abbott, J.C., Burhenne, H., Kaever, V., and Gründling, A. (2011) c-di-AMP Is a New Second Messenger in Staphylococcus aureus with a Role in Controlling Cell Size and Envelope Stress. PLoS Pathog 7: e1002217.

Corrigan, R.M., Bellows, L.E., Wood, A., and Gründling, A. (2016) ppGpp negatively impacts ribosome assembly affecting growth and antimicrobial tolerance in Gram-positive bacteria. Proc Natl Acad Sci 113: E1710-E1719.

Corrigan, R.M., Bowman, L., Willis, A.R., Kaever, V., and Gründling, A. (2015) Cross-talk between Two Nucleotide-signaling Pathways in Staphylococcus aureus. J Biol Chem 290: 5826-5839.

Corrigan, R.M., Campeotto, I., Jeganathan, T., Roelofs, K.G., Lee, V.T., et al. (2013) Systematic identification of conserved bacterial c-di-AMP receptor proteins. Proc Natl Acad Sci U S A 110: 9084-9089.

Corrigan, R.M., and Gründling, A. (2013) Cyclic di-AMP: Another second messenger enters the fray. Nat Rev Microbiol 11: 513-524.

Coutts, G. (2002) Membrane sequestration of the signal transduction protein GInK by the ammonium transporter AmtB. EMBO J 21: 536-545.

Danchin, A., and Nikel, P.I. (2019) Why Nature Chose Potassium. J Mol Evol 87: 271-288.

Dann, C.E., Wakeman, C.A., Sieling, C.L., Baker, S.C., Irnov, I., et al. (2007) Structure and Mechanism of a Metal-Sensing Regulatory RNA. Cell 130: 878-892.

Davies, B.W., Bogard, R.W., Young, T.S., and Mekalanos, J.J. (2012) Coordinated regulation of accessory genetic elements produces cyclic di-nucleotides for V. cholerae virulence. Cell 149: 358-370.

Detsch, C., and Stülke, J. (2003) Ammonium utilization in Bacillus subtilis: transport and regulatory functions of NrgA and NrgB. Microbiology 149: 3289-3297.

Devaux, L., Kaminski, P., Trieu-Cuot, P., and Firon, A. (2018a) Cyclic di-AMP in host-pathogen interactions. Curr Opin Microbiol 41: 21-28.

Devaux, L., Sleiman, D., Mazzuoli, M.-V., Gominet, M., Lanotte, P., et al. (2018b) Cyclic di-AMP regulation of osmotic homeostasis is essential in Group B Streptococcus. PLOS Genet 14: e1007342. 
Diethmaier, C., Newman, J.A., Kovacs, A.T., Kaever, V., Herzberg, C., et al. (2014) The YmdB Phosphodiesterase Is a Global Regulator of Late Adaptive Responses in Bacillus subtilis. J Bacteriol 196: $265-275$.

Diethmaier, C., Pietack, N., Gunka, K., Wrede, C., Lehnik-Habrink, M., et al. (2011) A Novel Factor Controlling Bistability in Bacillus subtilis: the YmdB Protein Affects Flagellin Expression and Biofilm Formation. J Bacteriol 193: 5997-6007.

Dinnbier, U., Limpinsel, E., Schmid, R., and Bakker, E.P. (1988) Transient accumulation of potassium glutamate and its replacement by trehalose during adaptation of growing cells of Escherichia coli K-12 to elevated sodium chloride concentrations. Arch Microbiol 150: 348-357.

Diskowski, M., Mehdipour, A.R., Wunnicke, D., Mills, D.J., Mikusevic, V., et al. (2017) Helical jackknives control the gates of the double-pore $\mathrm{K}^{+}$uptake system KtrAB. Elife 6.

Diskowski, M., Mikusevic, V., Stock, C., and Hänelt, I. (2015) Functional diversity of the superfamily of $\mathrm{K}^{+}$transporters to meet various requirements. Biol Chem 396.

Doucette, C.D., Schwab, D.J., Wingreen, N.S., and Rabinowitz, J.D. (2011) $\alpha$-ketoglutarate coordinates carbon and nitrogen utilization via enzyme I inhibition. Nat Chem Biol 7: 894-901.

Dubina, M. V., Vyazmin, S.Y., Boitsov, V.M., Nikolaev, E.N., Popov, I.A., et al. (2013) Potassium lons are More Effective than Sodium Ions in Salt Induced Peptide Formation. Orig Life Evol Biosph 43: 109117.

Duerig, A., Abel, S., Folcher, M., Nicollier, M., Schwede, T., et al. (2009) Second messenger-mediated spatiotemporal control of protein degradation regulates bacterial cell cycle progression. Genes Dev 23: 93-104.

Durell, S.R., and Guy, H.R. (1999) Structural models of the KtrB, TrkH, and Trk1,2 symporters based on the structure of the KcsA K+ channel. Biophys J 77: 789-807.

Dziejman, M., Balon, E., Boyd, D., Fraser, C.M., Heidelberg, J.F., et al. (2002) Comparative genomic analysis of Vibrio cholerae: Genes that correlate with cholera endemic and pandemic disease. Proc Natl Acad Sci U S A 99: 1556-1561.

Elfwing, A., LeMarc, Y., Baranyi, J., and Ballagi, A. (2004) Observing Growth and Division of Large Numbers of Individual Bacteria by Image Analysis. Appl Environ Microbiol 70: 675-678.

Ennis, H.L., and Artman, M. (1972) Ribosome size distribution in extracts of potassium-depleted Escherichia coli. Biochem Biophys Res Commun 48: 161-168.

Epstein, W. (2003) The Roles and Regulation of Potassium in Bacteria. In Progress in Nucleic Acid Research and Molecular Biology. pp. 293-320.

Ereño-Orbea, J., Oyenarte, I., and Martínez-Cruz, L.A. (2013) CBS domains: Ligand binding sites and conformational variability. Arch Biochem Biophys 540: 70-81. 
Eymann, C., Homuth, G., Scharf, C., and Hecker, M. (2002) Bacillus subtilis functional genomics: global characterization of the stringent response by proteome and transcriptome analysis. J Bacteriol 184: 2500-2520.

Fenton, A.W., Johnson, T.A., and Holyoak, T. (2010) The pyruvate kinase model system, a cautionary tale for the use of osmolyte perturbations to support conformational equilibria in allostery. Protein Sci 19: 1796-1800.

Firon, A., Dinis, M., Raynal, B., Poyart, C., Trieu-Cuot, P., et al. (2014) Extracellular nucleotide catabolism by the Group B Streptococcus ectonucleotidase NudP increases bacterial survival in blood. J Biol Chem 289: 5479-5489.

Forchhammer, K., Irmler, A., Kloft, N., and Ruppert, U. (2004) PIl signalling in unicellular cyanobacteria: analysis of redox-signals and energy charge. Physiol Plant 120: 51-56.

Forchhammer, K., and Lüddecke, J. (2016) Sensory properties of the P II signalling protein family. FEBS J 283: 425-437.

Forchhammer, K., and Tandeau de Marsac, N. (1995) Phosphorylation of the PIl protein ( $g / n B$ gene product) in the cyanobacterium Synechococcus sp. strain PCC 7942: analysis of in vitro kinase activity. J Bacteriol 177: 5812-5817.

French, C.T., Lao, P., Loraine, A.E., Matthews, B.T., Yu, H., et al. (2008) Large-scale transposon mutagenesis of Mycoplasma pulmonis. Mol Microbiol 69: 67-76.

Fujisawa, M., Ito, M., and Krulwich, T.A. (2007) Three two-component transporters with channel-like properties have monovalent cation/proton antiport activity. Proc Natl Acad Sci U S A 104: 1328913294

Fujisawa, M., Kusumoto, A., Wada, Y., Tsuchiya, T., and Ito, M. (2005) NhaK, a novel monovalent cation/ $\mathrm{H}^{+}$antiporter of Bacillus subtilis. Arch Microbiol 183: 411-420.

Gaballa, A., Antelmann, H., Aguilar, C., Khakh, S.K., Song, K.-B., et al. (2008) The Bacillus subtilis ironsparing response is mediated by a Fur-regulated small RNA and three small, basic proteins. Proc Natl Acad Sci 105: 11927-11932.

Gallagher, L.A., Bailey, J., and Manoil, C. (2020) Ranking essential bacterial processes by speed of mutant death. Proc Natl Acad Sci 117: 18010-18017.

Galperin, M. (2018) What bacteria want. Environ Microbiology 20:4221-4229.

Gao, A., and Serganov, A. (2014) Structural insights into recognition of c-di-AMP by the ydaO riboswitch. Nat Chem Biol 10: 787-792.

Garza-Ramos, G., Mújica-Jiménez, C., and Muñoz-Clares, R.A. (2013) Potassium and lonic Strength Effects on the Conformational and Thermal Stability of Two Aldehyde Dehydrogenases Reveal Structural and Functional Roles of $\mathrm{K}^{+}$-Binding Sites. PLoS One 8: e54899. 
Ger, M.-F., Rendon, G., Tilson, J.L., and Jakobsson, E. (2010) Domain-Based Identification and Analysis of Glutamate Receptor Ion Channels and Their Relatives in Prokaryotes. PLoS One 5: e12827.

Germain, E., Castro-Roa, D., Zenkin, N., and Gerdes, K. (2013) Molecular Mechanism of Bacterial Persistence by HipA. Mol Cell 52: 248-254.

Germain, E., Guiraud, P., Byrne, D., Douzi, B., Djendli, M., et al. (2019) YtfK activates the stringent response by triggering the alarmone synthetase SpoT in Escherichia coli. Nat Commun 10: 1-12.

Gerwig, J. (2014) Control of biofilm formation in Bacillus subtilis. Dissertation.

Gibhardt, J., Heidemann, J.L., Bremenkamp, R., Rosenberg, J., Seifert, R., et al. (2020) An extracytoplasmic protein and a moonlighting enzyme modulate synthesis of c-di-AMP in Listeria monocytogenes. Environ Microbiol 22: 2771-2791.

Gibhardt, J., Hoffmann, G., Turdiev, A., Wang, M., Lee, V.T., et al. (2019) c-di-AMP assists osmoadaptation by regulating the Listeria monocytogenes potassium transporters KimA and KtrCD. J Biol Chem 294: 16020-16033.

The GIMP Development Team (2019) GIMP; Retrieved from https://www.gimp.org.

Glass, J.I., Assad-Garcia, N., Alperovich, N., Yooseph, S., Lewis, M.R., et al. (2006) Essential genes of a minimal bacterium. Proc Natl Acad Sci U S A 103: 425-430.

Glass, T.L., Holmes, W.M., Hylemon, P.B., and Stellwag, E.J. (1979) Synthesis of guanosine tetra- and pentaphosphates by the obligately anaerobic bacterium Bacteroides thetaiotaomicron in response to molecular oxygen. J Bacteriol 137: 956-962.

Gohara, D.W., and Cera, E. Di (2016) Molecular mechanisms of enzyme activation by monovalent cations. J Biol Chem 291: 20840-20848.

Gomelsky, M. (2011) cAMP, c-di-GMP, c-di-AMP and now cGMP: Bacteria use them all! Mol Microbiol 79: 562-565.

Gonzalez, D., and Collier, J. (2014) Effects of (p)ppGpp on the progression of the cell cycle of Caulobacter crescentus. J Bacteriol 196: 2514-2525.

Goodwin, R.A., and Gage, D.J. (2014) Biochemical characterization of a nitrogen-type phosphotransferase system reveals that enzyme $\mathrm{El}^{\mathrm{Ntr}}$ integrates carbon and nitrogen signaling in Sinorhizobium meliloti. J Bacteriol 196: 1901-1907.

Goudreau, P., Stock, A. (1998) Signal transduction in bacteria: Molecular mechanisms of stimulusresponse coupling. Current Opinion in Microbiology 2: 160-169.

Görke, B., and Stülke, J. (2008) Carbon catabolite repression in bacteria: Many ways to make the most out of nutrients. Nat Rev Microbiol 6: 613-624.

Gries, C.M., Bose, J.L., Nuxoll, A.S., Fey, P.D., and Bayles, K.W. (2013) The Ktr potassium transport system in Staphylococcus aureus and its role in cell physiology, antimicrobial resistance and 
pathogenesis. Mol Microbiol 89: 760-773.

Gropp, M., Strausz, Y., Gross, M., and Glaser, G. (2001) Regulation of Escherichia coli RelA Requires Oligomerization of the C-Terminal Domain. J Bacteriol 183: 570-579.

Gründling, A. (2013) Potassium Uptake Systems in Staphylococcus aureus: New Stories about Ancient Systems. MBio 4.

Gundlach, J. (2017) Cyclic di-AMP signaling in Bacillus subtilis. Dissertation.

Gundlach, J., Commichau, F.M., and Stülke, J. (2018) Perspective of ions and messengers: an intricate link between potassium, glutamate, and cyclic di-AMP. Curr Genet 64: 191-195.

Gundlach, J., Dickmanns, A., Schröder-Tittmann, K., Neumann, P., Kaesler, J., et al. (2015a) Identification, Characterization, and Structure Analysis of the Cyclic di-AMP-binding P II -like Signal Transduction Protein DarA. J Biol Chem 290: 3069-3080.

Gundlach, J., Herzberg, C., Hertel, D., Thürmer, A., Daniel, R., et al. (2017a) Adaptation of Bacillus subtilis to Life at Extreme Potassium Limitation. MBio 8.

Gundlach, J., Herzberg, C., Kaever, V., Gunka, K., Hoffmann, T., et al. (2017b) Control of potassium homeostasis is an essential function of the second messenger cyclic di-AMP in Bacillus subtilis. Sci Signal 10: eaal3011.

Gundlach, J., Krüger, L., Herzberg, C., Turdiev, A., Poehlein, A., et al. (2019) Sustained sensing in potassium homeostasis: Cyclic di-AMP controls potassium uptake by KimA at the levels of expression and activity. J Biol Chem 294: 9605-9614.

Gundlach, J., Mehne, F.M.P., Herzberg, C., Kampf, J., Valerius, O., et al. (2015b) An essential poison: Synthesis and degradation of cyclic Di-AMP in Bacillus subtilis. J Bacteriol 197: 3265-3274.

Gunka, K. (2010) Der Einfluss der Glutamatdehydrogenasen auf die Verknüpfung des Kohlenstoff- und Stickstoffstoffwechsels in Bacillus subtilis. Dissertation.

Gunka, K., and Commichau, F.M. (2012) Control of glutamate homeostasis in Bacillus subtilis: A complex interplay between ammonium assimilation, glutamate biosynthesis and degradation. Mol Microbiol .

Gyan, S., Shiohira, Y., Sato, I., Takeuchi, M., and Sato, T. (2006) Regulatory Loop between Redox Sensing of the NADH/NAD ${ }^{+}$Ratio by Rex (YdiH) and Oxidation of NADH by NADH Dehydrogenase Ndh in Bacillus subtilis. J Bacteriol 188: 7062-7071.

Hahn, J., Tanner, A.W., Carabetta, V.J., Cristea, I.M., and Dubnau, D. (2015) ComGA-RelA interaction and persistence in the Bacillus subtilis K-state. Mol Microbiol 97: 454-471.

Halpern, Y.S., and Lupo, M. (1965) Glutamate transport in wild-type and mutant strains of Escherichia coli. J Bacteriol 90: 1288-95.

Hauryliuk, V., Atkinson, G.C., Murakami, K.S., Tenson, T., and Gerdes, K. (2015) Recent functional 
insights into the role of (p)ppGpp in bacterial physiology. Nat Rev Microbiol 13: 298-309.

He, H., Cooper, J.N., Mishra, A., and Raskin, D.M. (2012) Stringent response regulation of biofilm formation in Vibrio cholerae. J Bacteriol 194: 2962-2972.

He, J., Yin, W., Galperin, M.Y., and Chou, S.-H. (2020) Cyclic di-AMP, a second messenger of primary importance: tertiary structures and binding mechanisms. Nucleic Acids Res 48: 2807-2829.

Heidemann, J.L. (2020) Structural and biochemical characterization of c-di-AMP synthesizing enzymes. Dissertation.

Hengge, R., Häussler, S., Pruteanu, M., Stülke, J., Tschowri, N., et al. (2019) Recent Advances and Current Trends in Nucleotide Second Messenger Signaling in Bacteria. J Mol Biol 431: 908-927.

Herzberg, C., Weidinger, L.A.F., Dörrbecker, B., Hübner, S., Stülke, J., et al. (2007) SPINE: A method for the rapid detection and analysis of protein-protein interactions in vivo. Proteomics 7: 4032-4035.

Hoffmann, T., Boiangiu, C., Moses, S., and Bremer, E. (2008) Responses of Bacillus subtilis to Hypotonic Challenges: Physiological Contributions of Mechanosensitive Channels to Cellular Survival. Appl Environ Microbiol 74: 2454-2460.

Hofmeister, F. (1888) Zur Lehre von der Wirkung der Salze. Arch für Exp Pathol und Pharmakologie 25: $1-30$.

Hogg, T., Mechold, U., Malke, H., Cashel, M., and Hilgenfeld, R. (2004) Conformational antagonism between opposing active sites in a bifunctional RelA/SpoT homolog modulates ( $p$ )ppGpp metabolism during the stringent response. Cell 117: 57-68.

Holtmann, G., Bakker, E.P., Uozumi, N., and Bremer, E. (2003) KtrAB and KtrCD: Two K+ Uptake Systems in Bacillus subtilis and Their Role in Adaptation to Hypertonicity. J Bacteriol 185: 1289-1298.

Huang, K.C. (2017) Staying in Touch while on the Go. Cell 168: 15-17.

Hud, N. V., and Polak, M. (2001) DNA-cation interactions: The major and minor grooves are flexible ionophores. Curr Opin Struct Biol 11: 293-301.

Hutchison, C.A. (1999) Global Transposon Mutagenesis and a Minimal Mycoplasma Genome. Science 286: 2165-2169.

Huynh, T.N., Choi, P.H., Sureka, K., Ledvina, H.E., Campillo, J., et al. (2016) Cyclic di-AMP targets the cystathionine beta-synthase domain of the osmolyte transporter OpuC. Mol Microbiol 102: 233243.

Huynh, T.N., Luo, S., Pensinger, D., Sauer, J.-D., Tong, L., et al. (2015) An HD-domain phosphodiesterase mediates cooperative hydrolysis of c-di-AMP to affect bacterial growth and virulence. Proc Natl Acad Sci 112: E747-E756.

Ihara, Y., Ohta, H., and Masuda, S. (2015) A highly sensitive quantification method for the accumulation of alarmone ppGpp in Arabidopsis thaliana using UPLC-ESI-qMS/MS. J Plant Res 128: 511-518. 
Ikeda, T.P., Shauger, A.E., and Kustu, S. (1996) Salmonella typhimurium apparently perceives external nitrogen limitation as internal glutamine limitation. J Mol Bio/ 259: 589-607.

Jahn, N., Brantl, S., and Strahl, H. (2015) Against the mainstream: the membrane-associated type I toxin BsrG from Bacillus subtilis interferes with cell envelope biosynthesis without increasing membrane permeability. Mol Microbiol 98: 651-666.

Jenal, U., Reinders, A., and Lori, C. (2017) Cyclic di-GMP: second messenger extraordinaire. Nat Rev Microbiol 15: 271-284.

Jeong, B.C., Park, S.H., Yoo, K.S., Shin, J.S., and Song, H.K. (2013) Change in single cystathionine $\beta$ synthase domain-containing protein from a bent to flat conformation upon adenosine monophosphate binding. J Struct Biol 183: 40-46.

Jiang, Y., Lee, A., Chen, J., Cadene, M., Chait, B.T., et al. (2002) Crystal structure and mechanism of a calcium-gated potassium channel. Nature 417: 515-522.

Jiang, Y., Pico, A., Cadene, M., Chait, B.T., and MacKinnon, R. (2001) Structure of the RCK Domain from the E. coli $\mathrm{K}^{+}$Channel and Demonstration of Its Presence in the Human BK Channel. Neuron 29: 593-601.

Jin, X., Townley, R., and Shapiro, L. (2007) Structural Insight into AMPK Regulation: ADP Comes into Play. Structure 15: 1285-1295.

Jourlin-Castelli, C., Mani, N., Nakano, M.M., and Sonenshein, A.L. (2000) CcpC, a novel regulator of the LysR family required for glucose repression of the citB gene in Bacillus subtilis. J Mol Biol 295: 865-878.

Kampf, J. (2018) Insights into the biofilm formation of Bacillus subtilis. Dissertation.

Kampf, J., Gerwig, J., Kruse, K., Cleverley, R., Dormeyer, M., et al. (2018) Selective Pressure for Biofilm Formation in Bacillus subtilis: Differential Effect of Mutations in the Master Regulator SinR on Bistability. MBio 9.

Kampf, J., Gundlach, J., Herzberg, C., Treffon, K., and Stülke, J. (2017) Identification of c-di-AMP-Binding Proteins Using Magnetic Beads. c-di-GMP Signaling: Methods and Protocols, Methods in Molecular Biology. 1657: 347-359.

Kappes, R.M., Kempf, B., Kneip, S., Boch, J., Gade, J., et al. (1999) Two evolutionarily closely related $A B C$ transporters mediate the uptake of choline for synthesis of the osmoprotectant glycine betaine in Bacillus subtilis. Mol Microbiol 32: 203-216.

Karimova, G., Pidoux, J., Ullmann, A., and Ladant, D. (1998) A bacterial two-hybrid system based on a reconstituted signal transduction pathway. Proc Natl Acad Sci 95: 5752-5756.

Karstens, K., Zschiedrich, C.P., Bowien, B., Stülke, J., and Görke, B. (2014) Phosphotransferase protein EllANtr interacts with SpoT, a key enzyme of the stringent response, in Ralstonia eutropha $\mathrm{H} 16$. 
Microbiology 160: 711-722.

Kazlauskiene, M., Kostiuk, G., Venclovas, Č., Tamulaitis, G., and Siksnys, V. (2017) A cyclic oligonucleotide signaling pathway in type III CRISPR-Cas systems. Science 357: 605-609.

Kearse, M., Moir, R., Wilson, A., Stones-Havas, S., Cheung, M., et al. (2012) Geneious Basic: An integrated and extendable desktop software platform for the organization and analysis of sequence data. Bioinformatics 28: 1647-1649.

Kelly, C.D., and Rahn, O. (1931) The growth rate of individual bacterial cells. J Bacteriol 13: 147-153.

Kempf, B., and Bremer, E. (1995) OpuA, an Osmotically Regulated Binding Protein-dependent Transport System for the Osmoprotectant Glycine Betaine in Bacillus subtilis. J Biol Chem 270: 16701-16713.

Kim, H., Youn, S.J., Kim, S.O., Ko, J., Lee, J.O., et al. (2015) Structural studies of potassium transport protein KtrA regulator of conductance of $\mathrm{K}^{+}(\mathrm{RCK}) \mathrm{C}$ domain in complex with cyclic diadenosine monophosphate (c-di-AMP). J Biol Chem 290: 16393-16402.

Klewing, A (2013) Genomminimierung in Bacillus subtilis: Konsequenzen des Verlustes des Citratzyklus, Bachelor thesis.

Klewing, A., Koo, B., Krüger, L., Poehlein, A., Reuß, D., et al. (2020) Resistance to serine in Bacillus subtilis: identification of the serine transporter $\mathrm{YbeC}$ and of a metabolic network that links serine and threonine metabolism. Environ Microbio/ 22: 3937-3949.

Kong, C., Zeng, W., Ye, S., Chen, L., Sauer, D.B., et al. (2012) Distinct gating mechanisms revealed by the structures of a multi-ligand gated $\mathrm{K}^{+}$channel. Elife 2012: 1-20.

Koo, B., Kritikos, G., Farelli, J.D., Allen, K.N., Typas, A., et al. (2017) Construction and Analysis of Two Genome-Scale Deletion Libraries for Bacillus subtilis Article. Cell Syst 4: 291-305.e7.

Krammer, T.K. (2017) Functional analysis of the c-di-AMP binding proteins DarA and YkuL in Bacillus subtilis. Master thesis.

Krásný, L., and Gourse, R.L. (2004) An alternative strategy for bacterial ribosome synthesis: Bacillus subtilis rRNA transcription regulation. EMBO J 23: 4473-4483.

Krauße, D., Hunold, K., Kusian, B., Lenz, O., Jörg, S., et al. (2009) Essential Role of the hprK Gene in Ralstonia eutropha H16. J Mol Microbiol Biotechnol 17: 146-152.

Kröning, N., Willenborg, M., Tholema, N., Hänelt, I., Schmid, R., et al. (2007) ATP Binding to the KTN/RCK subunit KtrA from the $\mathrm{K}^{+}$-uptake system KtrAB of Vibrio alginolyticus: Its role in the formation of the KtrAB complex and its requirement in vivo. J Biol Chem 282: 14018-14027.

Krüger, L., Herzberg, C., Rath, H., Pedreira, T., Poehlein, A., et al. (2020a) Essentiality of c-di-AMP in Bacillus subtilis: Bypassing mutations converge in potassium and glutamate homeostasis. bioRxiv Krüger, L., Herzberg, C., Warneke, R., Poehlein, A., Stautz, J., et al. (2020b) Two Ways To Convert a 
Low-Affinity Potassium Channel to High Affinity: Control of Bacillus subtilis KtrCD by Glutamate. J Bacteriol 202.

Krüger, L., Herzberg, C., Wicke, D., Bähre, H., Heidemann, J.L., et al. (2020c) A rendezvous of two second messengers: The c-di-AMP receptor protein DarB controls (p)ppGpp synthesis in Bacillus subtilis. bioRxiv.

Kundig, W., Ghosh, S., and Roseman, S. (1964) Phosphate bound to histidine in a protein as an intermediate in a novel phospho-transferase system. Proc Natl Acad Sci 52: 1067-1074.

Kurata, T., Nakanishi, S., Hashimoto, M., Taoka, M., Yamazaki, Y., et al. (2015) Novel Essential Gene Involved in 16S rRNA Processing in Escherichia coli. J Mol Biol 427: 955-965.

Larsson, J.T., Rogstam, A., and Wachenfeldt, C. von (2005) Coordinated patterns of cytochrome bd and lactate dehydrogenase expression in Bacillus subtilis. Microbiology 151: 3323-3335.

Latoscha, A., Drexler, D.J., Al-Bassam, M.M., Bandera, A.M., Kaever, V., et al. (2020) c-di-AMP hydrolysis by the phosphodiesterase $\mathrm{AtaC}$ promotes differentiation of multicellular bacteria. Proc Natl Acad Sci 117: 7392-7400.

Lazzarini, R.A., Cashel, M. (1971) On the regulation of guanosine tetraphosphate levels in stringent and relaxed strains of Escherichia coli. J Biol Chem 246: 4381-4385.

Lee, C.-R., Cho, S.-H., Yoon, M.-J., Peterkofsky, A., and Seok, Y.-J. (2007) Escherichia coli enzyme IIA ${ }^{\text {Ntr }}$ regulates the $\mathrm{K}^{+}$transporter TrkA. Proc Natl Acad Sci 104: 4124-4129.

Lee, C., Park, Y.-H., Kim, M., Kim, Y., Park, S., et al. (2013) Reciprocal regulation of the autophosphorylation of enzyme I Ntr by glutamine and $\alpha$-ketoglutarate in Escherichia coli. Mol Microbiol 88: 473-485.

Lee, J., Park, Y., and Seok, Y. (2018) Rsd balances (p)ppGpp level by stimulating the hydrolase activity of SpoT during carbon source downshift in Escherichia coli. Proc Natl Acad Sci 115: E6845-E6854.

Lehnik-Habrink, M., Newman, J., Rothe, F.M., Solovyova, A.S., Rodrigues, C., et al. (2011) RNase Y in Bacillus subtilis: a Natively Disordered Protein That Is the Functional Equivalent of RNase E from Escherichia coli. J Bacteriol 193: 5431-5441.

Liu, G., Song, G., Zhang, D., Zhang, D., Li, Z., et al. (2014) EF-G catalyzes tRNA translocation by disrupting interactions between decoding center and codon-anticodon duplex. Nat Struct Mol Biol 21: 817824.

Liu, K., Bittner, A.N., and Wang, J.D. (2015) Diversity in (p)ppGpp metabolism and effectors. Curr Opin Microbiol 24: 72-79.

Lubin, M., and Ennis, H.L. (1964) On the role of intracellular potassium in protein synthesis. BBA Spec Sect Nucleic Acids Relat Subj 80: 614-631.

Lucas, M., Encinar, J.A., Arribas, E.A., Oyenarte, I., García, I.G., et al. (2010) Binding of S-Methyl-5'- 
Thioadenosine and S-Adenosyl-I-Methionine to Protein MJ0100 Triggers an Open-to-Closed Conformational Change in Its CBS Motif Pair. J Mol Biol 396: 800-820.

Ludwig, H. (2002) Die Rolle von CcpA bei der Verknüpfung von Kohlenstoff- und Stickstoff-Stoffwechsel in Bacillus subtilis. Dissertation.

Luo, Y., and Helmann, J.D. (2012) Analysis of the role of Bacillus subtilis $\sigma \mathrm{M}$ in $\beta$-lactam resistance reveals an essential role for c-di-AMP in peptidoglycan homeostasis. Mol Microbiol 83: 623-639.

Lüttmann, D., Göpel, Y., and Görke, B. (2015) Cross-Talk between the Canonical and the NitrogenRelated Phosphotransferase Systems Modulates Synthesis of the KdpFABC Potassium Transporter in Escherichia coli. J Mol Microbiol Biotechnol 25: 168-177.

Lüttmann, D., Heermann, R., Zimmer, B., Hillmann, A., Rampp, I.S., et al. (2009) Stimulation of the potassium sensor KdpD kinase activity by interaction with the phosphotransferase protein IIA Ntr in Escherichia coli. Mol Microbiol 72: 978-994.

Maaß, S., Wachlin, G., Bernhardt, J., Eymann, C., Fromion, V., et al. (2014) Highly precise quantification of protein molecules per cell during stress and starvation responses in Bacillus subtilis. Mol Cell Proteomics 13: 2260-2276.

MacLellan, S.R., Wecke, T., and Helmann, J.D. (2008) A previously unidentified $\sigma$ factor and two accessory proteins regulate oxalate decarboxylase expression in Bacillus subtilis. Mol Microbiol 69: 954-967.

Mäder U, Nicolas P. (2012) Array-based approaches to bacterial transcriptome analysis. In Methods in Microbiology, 39:151-182.

Makman, R.S., and Sutherland, E.W. (1965) Adenosine 3',5'-phosphate in Escherichia coli. J Biol Chem 240: 1309-14.

Mandal, M., and Breaker, R.R. (2004) Gene regulation by riboswitches. Nat Rev Mol Cell Biol 5: 451463.

Manikandan, K., Sabareesh, V., Singh, N., Saigal, K., Mechold, U., et al. (2014) Two-Step Synthesis and Hydrolysis of Cyclic di-AMP in Mycobacterium tuberculosis. PLoS One 9: e86096.

Maracci, C., Peske, F., Dannies, E., Pohl, C., and Rodnina, M. V. (2014) Ribosome-induced tuning of GTP hydrolysis by a translational GTPase. Proc Natl Acad Sci U S A 111: 14418-14423.

Marden, J.N., Dong, Q., Roychowdhury, S., Berleman, J.E., and Bauer, C.E. (2011) Cyclic GMP controls Rhodospirillum centenum cyst development. Mol Microbiol 79: 600-615.

McDonough, K.A., and Rodriguez, A. (2012) The myriad roles of cyclic AMP in microbial pathogens: from signal to sword. Nat Rev Microbiol 10: 27-38.

McFarland, A.P., Luo, S., Ahmed-Qadri, F., Zuck, M., Thayer, E.F., et al. (2017) Sensing of Bacterial Cyclic Dinucleotides by the Oxidoreductase RECON Promotes NF-KB Activation and Shapes a 
Proinflammatory Antibacterial State. Immunity 46: 433-445.

McLaggan, D., Logan, T.M., Lynn, D.G., and Epstein, W. (1990) Involvement of $\mathrm{\gamma}$-glutamyl peptides in osmoadaptation of Escherichia coli. J Bacteriol 172: 3631-3636.

McLaggan, D., Naprstek, J., Buurman, E.T., and Epstein, W. (1994) Interdependence of $\mathrm{K}^{+}$and glutamate accumulation during osmotic adaptation of Escherichia coli. J Biol Chem 269: 19111917.

Measures, J.C. (1975) Role of amino acids in osmoregulation of non-halophilic bacteria. Nature 257: 398-400.

Mechold, U., Murphy, H., Brown, L., and Cashel, M. (2002) Intramolecular Regulation of the Opposing (p)ppGpp Catalytic Activities of RelSeq, the Rel/Spo Enzyme from Streptococcus equisimilis. J Bacteriol 184: 2878-2888.

Mechold, U., Potrykus, K., Murphy, H., Murakami, K.S., and Cashel, M. (2013) Differential regulation by ppGpp versus pppGpp in Escherichia coli. Nucleic Acids Res 41: 6175-6189.

Mehne, F.M.P., Gunka, K., Eilers, H., Herzberg, C., Kaever, V., et al. (2013) Cyclic Di-AMP homeostasis in Bacillus subtilis: Both lack and high level accumulation of the nucleotide are detrimental for cell growth. J Biol Chem 288: 2004-2017.

Mehne, F.M.P., Schröder-Tittmann, K., Eijlander, R.T., Herzberg, C., Hewitt, L., et al. (2014) Control of the diadenylate cyclase CdaS in Bacillus subtilis: An autoinhibitory domain limits cyclic di-AMP production. J Biol Chem 289: 21098-21107.

Meinken, C., Blencke, H., Ludwig, H., and Stülke, J. (2003) Expression of the glycolytic gapA operon in Bacillus subtilis: differential syntheses of proteins encoded by the operon. Microbiology 149: 751-761.

Merrick, M. (2015) Post-translational modification of PII signal transduction proteins. Front Microbiol 6.

Merzbacher, M., Detsch, C., Hillen, W., and Stülke, J. (2004) Mycoplasma pneumoniae HPr kinase/phosphorylase: Assigning functional roles to the P-loop and the HPr kinase/phosphorylase signature sequence motif. Eur J Biochem 271: 367-374.

Meyer, F. (2012) Regulatory interactions of enzymes of the citric acid cycle in Bacillus subtilis. Dissertation.

Mikušević, V., Schrecker, M., Kolesova, N., Patiño-Ruiz, M., Fendler, K., et al. (2019) A channel profile report of the unusual K+ channel KtrB. J Gen Physiol 151: 1357-1368.

Miskin, R., Zamir, A., and Elson, D. (1970) Inactivation and reactivation of ribosomal subunits: The peptidyl transferase activity of the 50 s subunit of Escherichia coli. J Mol Biol 54: 355-378.

Monod, J. (1949) The Growth of Bacterial Cultures. Annu Rev Microbiol 3: 371-394. 
Moscoso, J.A., Schramke, H., Zhang, Y., Tosi, T., Dehbi, A., et al. (2016) Binding of cyclic di-AMP to the Staphylococcus aureus sensor kinase KdpD occurs via the universal stress protein domain and downregulates the expression of the Kdp potassium transporter. J Bacterio/ 198: 98-110.

Murray, H., and Koh, A. (2014) Multiple Regulatory Systems Coordinate DNA Replication with Cell Growth in Bacillus subtilis. PLoS Genet 10: e1004731.

Murray, H.D., Schneider, D.A., and Gourse, R.L. (2003) Control of rRNA Expression by Small Molecules Is Dynamic and Nonredundant. Mol Cell 12: 125-134.

Nakayama, Y., Hashimoto, K., Kawasaki, H., and Martinac, B. (2019) "Force-From-Lipids" mechanosensation in Corynebacterium glutamicum. Biophys Rev 11: 327-333.

Nakayama, Y., Yoshimura, K., and lida, H. (2012) A Gain-of-Function Mutation in Gating of Corynebacterium glutamicum NCgl1221 Causes Constitutive Glutamate Secretion. Appl Environ Microbiol 78: 5432-5434.

Nanamiya, H., Kasai, K., Nozawa, A., Yun, C.-S., Narisawa, T., et al. (2008) Identification and functional analysis of novel (p)ppGpp synthetase genes in Bacillus subtilis. Mol Microbiol 67: 291-304.

Nelson, J.W., and Breaker, R.R. (2017) The lost language of the RNA World. Sci Signal 10: 1-11.

Nelson, J.W., Sudarsan, N., Furukawa, K., Weinberg, Z., Wang, J.X., et al. (2013) Riboswitches in eubacteria sense the second messenger c-di-AMP. Nat Chem Biol 9: 834-839.

Nicolas, P., Mader, U., Dervyn, E., Rochat, T., Leduc, A., et al. (2012) Condition-Dependent Transcriptome Reveals High-Level Regulatory Architecture in Bacillus subtilis. Science 335: 11031106.

Niewoehner, O., Garcia-Doval, C., Rostøl, J.T., Berk, C., Schwede, F., et al. (2017) Type III CRISPR-Cas systems produce cyclic oligoadenylate second messengers. Nature 548: 543-548.

Nightingale, E.R. (1959) Phenomenological theory of ion solvation. Effective radii of hydrated ions. J Phys Chem 63: 1381-1387.

Ninfa, A.J., and Jiang, P. (2005) PII signal transduction proteins: sensors of $\alpha$-ketoglutarate that regulate nitrogen metabolism. Curr Opin Microbiol 8: 168-173.

Nisco, N.J. De, Abo, R.P., Wu, C.M., Penterman, J., and Walker, G.C. (2014) Global analysis of cell cycle gene expression of the legume symbiont Sinorhizobium meliloti. Proc Natl Acad Sci U S A 111: 3217-3224.

Nowak, T., and Suelter, C. (1981) Pyruvate kinase: Activation by and catalytic role of the monovalent and divalent cations. Mol Cell Biochem 35: 65-75.

Oppenheimer-Shaanan, Y., Wexselblatt, E., Katzhendler, J., Yavin, E., and Ben-Yehuda, S. (2011) c-diAMP reports DNA integrity during sporulation in Bacillus subtilis. EMBO Rep 12: 594-601.

Orr, M.W., Galperin, M.Y., and Lee, V.T. (2016) Sustained sensing as an emerging principle in second 
messenger signaling systems. Curr Opin Microbiol 34: 119-126.

Park, Y., Lee, C.-R., Choe, M., Seok, Y.-J. (2013) HPr antagonizes the anti- $\sigma^{70}$ activity of Rsd in Escherichia coli. Proc Natl Acad Sci U S A 110: 21142-21147.

Pausch, P., Abdelshahid, M., Steinchen, W., Schäfer, H., Gratani, F.L., et al. (2020) Structural Basis for Regulation of the Opposing (p)ppGpp Synthetase and Hydrolase within the Stringent Response Orchestrator Rel. Cell Rep 32: 108157.

Peng, B.-Z., Bock, L. V., Belardinelli, R., Peske, F., Grubmüller, H., et al. (2019) Active role of elongation factor $\mathrm{G}$ in maintaining the mRNA reading frame during translation. Sci Adv 5: eaax8030.

Perez-Riverol, Y., Csordas, A., Bai, J., Bernal-Llinares, M., Hewapathirana, S., et al. (2019) The PRIDE database and related tools and resources in 2019: Improving support for quantification data. Nucleic Acids Res 47: D442-D450.

Peterson, B.N., Young, M.K.M., Luo, S., Wang, J., Whiteley, A.T., et al. (2020) (p)ppGpp and c-di-AMP Homeostasis Is Controlled by CbpB in Listeria monocytogenes. MBio 11.

Pflüger-Grau, K., and Görke, B. (2010) Regulatory roles of the bacterial nitrogen-related phosphotransferase system. Trends Microbiol 18: 205-214.

Pflüger-Grau, K., and Lorenzo, V. de (2014) From the phosphoenolpyruvate phosphotransferase system to selfish metabolism: A story retraced in Pseudomonas putida. FEMS Microbiol Lett 356: 144-153.

Pham, H.T., Nhiep, N.T.H., Vu, T.N.M., Huynh, T.N., Zhu, Y., et al. (2018) Enhanced uptake of potassium or glycine betaine or export of cyclic-di-AMP restores osmoresistance in a high cyclic-di-AMP Lactococcus lactis mutant. PLOS Genet 14: e1007574.

Pietack, N. (2010) Investigation of glycolysis in Bacillus subtilis. Dissertation.

Pliotas, C., Dahl, A.C.E., Rasmussen, T., Mahendran, K.R., Smith, T.K., et al. (2015) The role of lipids in mechanosensation. Nat Struct Mol Biol 22: 991-998.

Pliotas, C., Grayer, S.C., Ekkerman, S., Chan, A.K.N., Healy, J., et al. (2017) Adenosine Monophosphate Binding Stabilizes the KTN Domain of the Shewanella denitrificans Kef Potassium Efflux System. Biochemistry 56: 4219-4234.

Poolman, B., Hellingwerf, K.J., and Konings, W.N. (1987) Regulation of the glutamate-glutamine transport system by intracellular pH in Streptococcus lactis. J Bacteriol 169: 2272-2276.

Postma, P.W., Lengeler, J.W., and Jacobson, G.R. (1993) Phosphoenolpyruvate: Carbohydrate phosphotransferase systems of bacteria. Microbiol Rev 57: 543-594.

Potrykus, K., and Cashel, M. (2008) (p)ppGpp: Still Magical? Annu Rev Microbiol 62: 35-51.

Powell, B.S., Court, D.L., Inada, T., Nakamura, Y., Michotey, V., et al. (1995) Novel Proteins of the Phosphotransferase System Encoded within the rpoN Operon of Escherichia coli. J Biol Chem 270: 
4822-4839.

Presecan-Siedel, E., Galinier, A., Longin, R., Deutscher, J., Danchin, A., et al. (1999) Catabolite Regulation of the pta Gene as Part of Carbon Flow Pathways in Bacillus subtilis. J Bacteriol 181: 6889-6897.

Price-Whelan, A., Poon, C.K., Benson, M.A., Eidem, T.T., Roux, C.M., et al. (2013) Transcriptional Profiling of Staphylococcus aureus During Growth in $2 \mathrm{M} \mathrm{NaCl}$ Leads to Clarification of Physiological Roles for Kdp and Ktr K+ Uptake Systems. MBio 4.

Prindle, A., Liu, J., Asally, M., Ly, S., Garcia-Ojalvo, J., et al. (2015) Ion channels enable electrical communication in bacterial communities. Nature 527: 59-63.

Qin, Y., He, Y., She, Q., Larese-Casanova, P., Li, P., et al. (2019) Heterogeneity in respiratory electron transfer and adaptive iron utilization in a bacterial biofilm. Nat Commun 10: 3702.

Quintana, I.M., Gibhardt, J., Turdiev, A., Hammer, E., Commichau, F.M., et al. (2019) The KupA and KupB Proteins of Lactococcus lactis IL1403 Are Novel c-di-AMP Receptor Proteins Responsible for Potassium Uptake. J Bacteriol 201.

Quintero, F.J., and Blatt, M.R. (1997) A new family of $\mathrm{K}^{+}$transporters from Arabidopsis that are conserved across phyla. FEBS Lett 415: 206-211.

Rabus, R., Reizer, J., Paulsen, I., and Saier, M.H. (1999) Enzyme I Ntr from Escherichia coli. J Biol Chem 274: 26185-26191.

Radchenko, M. V., Tanaka, K., Waditee, R., Oshimi, S., Matsuzaki, Y., et al. (2006) Potassium/Proton Antiport System of Escherichia coli. J Biol Chem 281: 19822-19829.

Ramesh, A., and Winkler, W.C. (2010) Magnesium-sensing riboswitches in bacteria. RNA Biol 7.

Rancati, G., Moffat, J., Typas, A., and Pavelka, N. (2018) Emerging and evolving concepts in gene essentiality. Nat Rev Genet 19: 34-49.

Rao, F., See, R.Y., Zhang, D., Toh, D.C., Ji, Q., et al. (2010) YybT is a signaling protein that contains a cyclic dinucleotide phosphodiesterase domain and a GGDEF domain with ATPase activity. J Biol Chem 285: 473-482.

Rappsilber, J., Ishihama, Y., and Mann, M. (2003) Stop And Go Extraction tips for matrix-assisted laser desorption/ionization, nanoelectrospray, and LC/MS sample pretreatment in proteomics. Anal Chem 75: 663-670.

Rappsilber, J., Mann, M., and Ishihama, Y. (2007) Protocol for micro-purification, enrichment, prefractionation and storage of peptides for proteomics using StageTips. Nat Protoc 2: 1896-1906.

Raskin, D.M., Judson, N., and Mekalanos, J.J. (2007) Regulation of the stringent response is the essential function of the conserved bacterial G protein CgtA in Vibrio cholerae. Proc Natl Acad Sci 104: 4636-4641. 
Rasmussen, T., Rasmussen, A., Yang, L., Kaul, C., Black, S., et al. (2019) Interaction of the Mechanosensitive Channel, MscS, with the Membrane Bilayer through Lipid Intercalation into Grooves and Pockets. J Mol Biol 431: 3339-3352.

Rehm, N., and Burkovski, A. (2011) Engineering of nitrogen metabolism and its regulation in Corynebacterium glutamicum: Influence on amino acid pools and production. Appl Microbiol Biotechnol 89: 239-248.

Reitzer, L. (2003) Nitrogen Assimilation and Global Regulation in Escherichia coli. Annu Rev Microbiol 57: 155-176.

Ren, A., and Patel, D.J. (2014) C-di-AMP binds the $y d a O$ riboswitch in two pseudo-symmetry-related pockets. Nat Chem Biol 10: 780-786.

Reuß, D.R., Altenbuchner, J., Mäder, U., Rath, H., Ischebeck, T., et al. (2017) Large-scale reduction of the Bacillus subtilis genome: Consequences for the transcriptional network, resource allocation, and metabolism. Genome Res 27: 289-299.

Reuß, D.R., Faßhauer, P., Mroch, P.J., Ul-Haq, I., Koo, B.-M., et al. (2019) Topoisomerase IV can functionally replace all type 1A topoisomerases in Bacillus subtilis. Nucleic Acids Res 47: 52315242.

Robertson, R.M., Yao, J., Gajewski, S., Kumar, G., Martin, E.W., et al. (2017) A two-helix motif positions the lysophosphatidic acid acyltransferase active site for catalysis within the membrane bilayer. Nat Struct Mol Biol 24: 666-671.

Rocha, R., Teixeira-duarte, C.M., Jorge, J.M.P., and Morais-cabral, J.H. (2019) Characterization of the molecular properties of KtrC, a second RCK domain that regulates a Ktr channel in Bacillus subtilis. J Struct Biol 205: 34-43.

Roelofs, K.G., Wang, J., Sintim, H.O., and Lee, V.T. (2011) Differential radial capillary action of ligand assay for high-throughput detection of protein-metabolite interactions. Proc Natl Acad Sci 108: 15528-15533.

Römling, U. (2008) Great times for small molecules: C-di-AMP, a second messenger candidate in bacteria and archaea. Sci Signal 1: 1-5.

Ronneau, S., Caballero-Montes, J., Coppine, J., Mayard, A., Garcia-Pino, A., et al. (2019) Regulation of (p)ppGpp hydrolysis by a conserved archetypal regulatory domain. Nucleic Acids Res 47: 843854.

Ronneau, S., and Hallez, R. (2019) Make and break the alarmone: regulation of (p)ppGpp synthetase/hydrolase enzymes in bacteria. FEMS Microbiol Rev 43: 389-400.

Ronneau, S., Petit, K., Bolle, X. De, and Hallez, R. (2016) Phosphotransferase-dependent accumulation of (p)ppGpp in response to glutamine deprivation in Caulobacter crescentus. Nat Commun 7: 
11423.

Roosild, T.P., Miller, S., Booth, I.R., and Choe, S. (2002) A mechanism of regulating transmembrane potassium flux through a ligand-mediated conformational switch. Cell 109: 781-791.

Rosenberg, J., Dickmanns, A., Neumann, P., Gunka, K., Arens, J., et al. (2015) Structural and Biochemical Analysis of the Essential Diadenylate Cyclase CdaA from Listeria monocytogenes. J Biol Chem 290: 6596-6606.

Rosenberg, J., Müller, P., Lentes, S., Thiele, M.J., Zeigler, D.R., et al. (2016) ThrR, a DNA-binding transcription factor involved in controlling threonine biosynthesis in Bacillus subtilis. Mol Microbiol 101: 879-893.

Ross, P., Weinhouse, H., Aloni, Y., Michaeli, D., Weinberger-Ohana, P., et al. (1987) Regulation of cellulose synthesis in Acetobacter xylinum by cyclic diguanylic acid. Nature 325: 279-281.

Ross, W., Vrentas, C.E., Sanchez-Vazquez, P., Gaal, T., and Gourse, R.L. (2013) The magic spot: A ppGpp binding site on $E$. coli RNA polymerase responsible for regulation of transcription initiation. Mol Cell 50: 420-429.

Rozov, A., Khusainov, I., Omari, K. El, Duman, R., Mykhaylyk, V., et al. (2019) Importance of potassium ions for ribosome structure and function revealed by long-wavelength X-ray diffraction. Nat Commun 10: 1-12.

Rubin, B.E., Huynh, T.N., Welkie, D.G., Diamond, S., Simkovsky, R., et al. (2018) High-throughput interaction screens illuminate the role of c-di-AMP in cyanobacterial nighttime survival. PLOS Genet 14: e1007301.

Sambrook, J., and Russell, D. (2001) Molecular Cloning: A Laboratory Manual, Cold Spring Harbor Laboratory, Cold Spring Harbor, NY.

Santa-Maria, G.E., Rubio, F., Dubcovsky, J., and Rodriguez-Navarro, A. (1997) The HAK1 Gene of Barley Is a Member of a Large Gene Family and Encodes a High-Affinity Potassium Transporter. Plant Cell 9: 2281.

Sato, Y., Noji, S., Suzuki, R., and Taniguchi, S. (1989) Dual mechanism for stimulation of glutamate transport by potassium ions in Streptococcus mutans. J Bacteriol 171: 4963-4966.

Schäfer, H., Beckert, B., Frese, C.K., Steinchen, W., Nuss, A.M., et al. (2020) The alarmones (p)ppGpp are part of the heat shock response of Bacillus subtilis. PLOS Genet 16: e1008275.

Schirmer, F., Ehrt, S., and Hillen, W. (1997) Expression, inducer spectrum, domain structure, and function of MopR, the regulator of phenol degradation in Acinetobacter calcoaceticus NCIB8250. J Bacteriol 179: 1329-1336.

Schrecker, M., Wunnicke, D., and Hänelt, I. (2019) How RCK domains regulate gating of $\mathrm{K}^{+}$channels. Biol Chem 400: 1303-1322. 
Schujman, G.E., Paoletti, L., Grossman, A.D., and Mendoza, D. de (2003) FapR, a bacterial transcription factor involved in global regulation of membrane lipid biosynthesis. Dev Cell 4: 663-672.

Schumacher, M.A., Chinnam, N.B., Cuthbert, B., Tonthat, N.K., and Whitfill, T. (2015) Structures of regulatory machinery reveal novel molecular mechanisms controlling $B$. subtilis nitrogen homeostasis. Genes Dev 29: 451-464.

Schuster, C.F., Bellows, L.E., Tosi, T., Campeotto, I., Corrigan, R.M., et al. (2016) The second messenger c-di-AMP inhibits the osmolyte uptake system OpuC in Staphylococcus aureus. Sci Signal 9: ra81ra81.

Scott, J.W., Hawley, S.A., Green, K.A., Anis, M., Stewart, G., et al. (2004) CBS domains form energysensing modules whose binding of adenosine ligands is disrupted by disease mutations. J Clin Invest 113: 274-284.

Shannon, P., Markiel, A., Ozier, O., Baliga, N.S., Wang, J.T., et al. (2003) Cytoscape: a software environment for integrated models of biomolecular interaction networks. Genome Res 13: 2498504.

Shevchenko, A., Wilm, M., Vorm, O., and Mann, M. (1996) Mass spectrometric sequencing of proteins from silver-stained polyacrylamide gels. Anal Chem 68: 850-858.

Silvers, M.A., Pakhomova, S., Neau, D.B., Silvers, W.C., Anzalone, N., et al. (2016) Crystal Structure of Carboxyltransferase from Staphylococcus aureus Bound to the Antibacterial Agent Moiramide B. Biochemistry 55: 4666-4674.

Smith, W.M., Pham, T.H., Lei, L., Dou, J., Soomro, A.H., et al. (2012) Heat Resistance and Salt Hypersensitivity in Lactococcus lactis Due to Spontaneous Mutation of Ilmg_1816 (gdpP) Induced by High-Temperature Growth. Appl Environ Microbiol 78: 7753-7759.

Sobolevsky, A.I. (2015) Structure and gating of tetrameric glutamate receptors. J Physio/ 593: 29-38.

Song, J.H., Ko, K.S., Lee, J.Y., Baek, J.Y., Oh, W.S., et al. (2005) Identification of essential genes in Streptococcus pneumoniae by allelic replacement mutagenesis. Mol Cells 19: 365-374.

Steinchen, W., and Bange, G. (2016) The magic dance of the alarmones (p)ppGpp. Mol Microbiol 101: 531-544.

Steinchen, W., Zegarra, V., and Bange, G. (2020) (p)ppGpp: Magic Modulators of Bacterial Physiology and Metabolism. Front Microbiol 11.

Strovas, T.J., Sauter, L.M., Guo, X., and Lidstrom, M.E. (2007) Cell-to-Cell Heterogeneity in Growth Rate and Gene Expression in Methylobacterium extorquens AM1. J Bacteriol 189: 7127-7133.

Stülke, J., and Hillen, W. (1998) Coupling physiology and gene regulation in bacteria: The phosphotransferase sugar uptake system delivers the signals. Naturwissenschaften 85: 583-592.

Stülke, J., and Krüger, L. (2020) Cyclic di-AMP Signaling in Bacteria. Annu Rev Microbiol 74: 159-179. 
Stülke, J., Martin-Verstraete, I., Zagorec, M., Rose, M., Klier, A., et al. (1997) Induction of the Bacillus subtilis ptsGHI operon by glucose is controlled by a novel antiterminator, GlcT. Mol Microbiol 25: $65-78$.

Stumpe, S., and Bakker, E.P. (1997) Requirement of a large $\mathrm{K}^{+}$-uptake capacity and of extracytoplasmic protease activity for protamine resistance of Escherichia coli. Arch Microbiol 167: 126-136.

Sudarsan, N., Lee, E.R., Weinberg, Z., Moy, R.H., Kim, J.N., et al. (2008) Riboswitches in eubacteria sense the second messenger cyclic Di-GMP. Science 321: 411-413.

Sureka, K., Choi, P.H., Precit, M., Delince, M., Pensinger, D.A., et al. (2014) The Cyclic Dinucleotide cdi-AMP Is an Allosteric Regulator of Metabolic Enzyme Function. Cell 158: 1389-1401.

Szollosi, A., Vieira-Pires, R.S., Teixeira-Duarte, C.M., Rocha, R., and Morais-Cabral, J.H. (2016) Dissecting the Molecular Mechanism of Nucleotide-Dependent Activation of the KtrAB K ${ }^{+}$Transporter. PLOS Biol 14: e1002356.

Takada, H., Roghanian, M., Murina, V., Dzhygyr, I., Murayama, R., et al. (2020) The C-Terminal RRM/ACT Domain Is Crucial for Fine-Tuning the Activation of 'Long' RelA-SpoT Homolog Enzymes by Ribosomal Complexes. Front Microbiol 11.

Tamayo, R., Pratt, J.T., and Camilli, A. (2007) Roles of Cyclic Diguanylate in the Regulation of Bacterial Pathogenesis. Annu Rev Microbiol 61: 131-148.

Tascón, I., Sousa, J.S., Corey, R.A., Mills, D.J., Griwatz, D., et al. (2020) Structural basis of proton-coupled potassium transport in the KUP family. Nat Commun 11: 1-10.

Terakawa, A., Natsume, A., Okada, A., Nishihata, S., Kuse, J., et al. (2016) Bacillus subtilis 5'nucleotidases with various functions and substrate specificities. BMC Microbiol 16: 249.

Tesch, M., Graaf, A.A. De, and Sahm, H. (1999) In vivo fluxes in the ammonium-assimilatory pathways in Corynebacterium glutamicum studied by ${ }^{15} \mathrm{~N}$ nuclear magnetic resonance. Appl Environ Microbiol 65: 1099-1109.

Tholema, N., Brüggen, M.V. der, Mäser, P., Nakamura, T., Schroeder, J.I., et al. (2005) All Four Putative Selectivity Filter Glycine Residues in KtrB Are Essential for High Affinity and Selective $\mathrm{K}^{+}$Uptake by the KtrAB System from Vibrio alginolyticus. J Biol Chem 280: 41146-41154.

Tödter, D., Gunka, K., and Stülke, J. (2017) The Highly Conserved Asp23 Family Protein YqhY Plays a Role in Lipid Biosynthesis in Bacillus subtilis. Front Microbiol 8.

Ullmann, A., and Monod, J. (1968) Cyclic AMP as an antagonist of catabolite repression in Escherichia coli. FEBS Lett 2: 57-60.

Vastermark, A., Wollwage, S., Houle, M.E., Rio, R., and Saier, M.H. (2014) Expansion of the APC superfamily of secondary carriers. Proteins Struct Funct Bioinforma 82: 2797-2811.

Vieira-Pires, R.S., Szollosi, A., and Morais-Cabral, J.H. (2013) The structure of the KtrAB potassium 
transporter. Nature 496: 323-328.

Vrbka, L., Vondrášek, J., Jagoda-Cwiklik, B., Vácha, R., and Jungwirth, P. (2006) Quantification and rationalization of the higher affinity of sodium over potassium to protein surfaces. Proc Natl Acad Sci U S A 103: 15440-15444.

Wacker, I., Ludwig, H., Reif, I., Blencke, H., Detsch, C., et al. (2003) The regulatory link between carbon and nitrogen metabolism in Bacillus subtilis: regulation of the gltAB operon by the catabolite control protein CcpA. Microbiology 149: 3001-3009.

Wahome, P.G., and Setlow, P. (2007) Growth, osmotic downshock resistance and differentiation of Bacillus subtilis strains lacking mechanosensitive channels. Arch Microbiol 189: 49-58.

Wakeman, C.A., Goodson, J.R., Zacharia, V.M., and Winkler, W.C. (2014) Assessment of the Requirements for Magnesium Transporters in Bacillus subtilis. J Bacteriol 196: 1206-1214.

Wang, Y., Cao, G., Xu, D., Fan, L., Wu, X., et al. (2018) A Novel Corynebacterium glutamicum LGlutamate Exporter. Appl Environ Microbiol 84: 1-15.

Waters, C.M., Lu, W., Rabinowitz, J.D., and Bassler, B.L. (2008) Quorum sensing controls biofilm formation in Vibrio cholerae through modulation of cyclic Di-GMP levels and repression of vpsT. J Bacteriol 190: 2527-2536.

Weiß, M. (2018) A protein in search of a function: The c-di-AMP-binding protein DarA of Bacillus subtilis. Dissertation.

Wells, D.H., and Gaynor, E.C. (2006) Helicobacter pylori initiates the stringent response upon nutrient and $\mathrm{pH}$ downshift. J Bacteriol 188: 3726-3729.

Whatmore, A.M., Chudek, J.A., and Reed, R.H. (1990) The effects of osmotic upshock on the intracellular solute pools of Bacillus subtilis. J Gen Microbiol 136: 2527-2535.

Whiteley, A.T., Eaglesham, J.B., Oliveira Mann, C.C. de, Morehouse, B.R., Lowey, B., et al. (2019) Bacterial cGAS-like enzymes synthesize diverse nucleotide signals. Nature 567: 194-199.

Whiteley, A.T., Garelis, N.E., Peterson, B.N., Choi, P.H., Tong, L., et al. (2017) c-di-AMP modulates Listeria monocytogenes central metabolism to regulate growth, antibiotic resistance and osmoregulation. Mol Microbiol 104: 212-233.

Whiteley, A.T., Pollock, A.J., and Portnoy, D.A. (2015) The PAMP c-di-AMP Is Essential for Listeria monocytogenes Growth in Rich but Not Minimal Media due to a Toxic Increase in (p)ppGpp. Cell Host Microbe 17: 788-798.

Witte, C.E., Whiteley, A.T., Burke, T.P., Sauer, J.-D., Portnoy, D.A., et al. (2013) Cyclic di-AMP Is Critical for Listeria monocytogenes Growth, Cell Wall Homeostasis, and Establishment of Infection. MBio 4: $1-10$.

Witte, G., Hartung, S., Büttner, K., and Hopfner, K.P. (2008) Structural Biochemistry of a Bacterial 
Checkpoint Protein Reveals Diadenylate Cyclase Activity Regulated by DNA Recombination Intermediates. Mol Cell 30: 167-178.

Wood, J.M. (1999) Osmosensing by Bacteria: Signals and Membrane-Based Sensors. Microbiol Mol Biol Rev 63: 230-262.

Wood, J.M. (2011) Bacterial Osmoregulation: A Paradigm for the Study of Cellular Homeostasis. Annu Rev Microbiol 65: 215-238.

Woodward, J.J., Lavarone, A.T., and Portnoy, D.A. (2010) C-di-AMP secreted by intracellular Listeria monocytogenes activates a host type I interferon response. Science 328: 1703-1705.

Wout, P., Pu, K., Sullivan, S.M., Reese, V., Zhou, S., et al. (2004) The Escherichia coli GTPase CgtAE Cofractionates with the $50 \mathrm{~S}$ Ribosomal Subunit and Interacts with SpoT, a ppGpp Synthetase/Hydrolase. J Bacteriol 186: 5249-5257.

Xiao, B., Heath, R., Saiu, P., Leiper, F.C., Leone, P., et al. (2007) Structural basis for AMP binding to mammalian AMP-activated protein kinase. Nature 449: 496-500.

Xie, W.J., and Gao, Y.Q. (2013) A simple theory for the hofmeister series. J Phys Chem Lett 4: 42474252.

$\mathrm{Xu}$, J., and Zhang, Y. (2010) How significant is a protein structure similarity with TM-score $=0.5$ ? Bioinformatics 26: 889-895.

Yang, X., and Ishiguro, E.E. (2001) Dimerization of the RelA protein of Escherichia coli. Biochem Cell Biol 79: 729-736.

Ye, M., Zhang, J.J., Fang, X., Lawlis, G.B., Troxell, B., et al. (2014) DhhP, a cyclic di-AMP phosphodiesterase of Borrelia burgdorferi, is essential for cell growth and virulence. Infect Immun 82: $1840-1849$.

Yuan, A., Santi, C.M., Wei, A., Wang, Z.-W., Pollak, K., et al. (2003) The Sodium-Activated Potassium Channel Is Encoded by a Member of the Slo Gene Family. Neuron 37: 765-773.

Zaprasis, A., Bleisteiner, M., Kerres, A., Hoffmann, T., and Bremer, E. (2015) Uptake of amino acids and their metabolic conversion into the compatible solute proline confers osmoprotection to Bacillus subtilis. Appl Environ Microbiol 81: 250-259.

Zarrella, T.M., Metzger, D.W., and Bai, G. (2018) Stress Suppressor Screening Leads to Detection of Regulation of Cyclic di-AMP Homeostasis by a Trk Family Effector Protein in Streptococcus pneumoniae. J Bacteriol 200.

Zeden, M.S., Kviatkovski, I., Schuster, C.F., Thomas, V.C., Fey, P.D., et al. (2020) Identification of the main glutamine and glutamate transporters in Staphylococcus aureus and their impact on c-diAMP production. Mol Microbiol 113: 1085-1100.

Zeden, M.S., Schuster, C.F., Bowman, L., Zhong, Q., Williams, H.D., et al. (2018) Cyclic di-adenosine 
monophosphate (c-di-AMP) is required for osmotic regulation in Staphylococcus aureus but dispensable for viability in anaerobic conditions. J Biol Chem 293: 3180-3200.

Zhang, J.-J., Chen, T., Yang, Y., Du, J., Li, H., et al. (2018) Positive and Negative Regulation of Glycerol Utilization by the c-di-GMP Binding Protein PIzA in Borrelia burgdorferi. J Bacteriol 200: 1-11.

Zhang, X., Solaro, C.R., and Lingle, C.J. (2001) Allosteric regulation of BK channel gating by $\mathrm{Ca}^{2+}$ and $\mathrm{Mg}^{2+}$ through a nonselective, low affinity divalent cation site. J Gen Physiol 118: 607-635.

Zhang, X., Wang, J., Feng, Y., Ge, J., Li, W., et al. (2012) Structure and molecular mechanism of an anionselective mechanosensitive channel of small conductance. Proc Nat/ Acad Sci 109: 18180-18185.

Zheng, C., Ma, Y., Wang, X., Xie, Y., Ali, M.K., et al. (2015) Functional analysis of the sporulation-specific diadenylate cyclase CdaS in Bacillus thuringiensis. Front Microbiol 6: 1-15.

Zhu, B., and Stülke, J. (2018) SubtiWiki in 2018: from genes and proteins to functional network annotation of the model organism Bacillus subtilis. Nucleic Acids Res 46: 743-748.

Zhu, Y., Pham, T.H., Nhiep, T.H.N., Vu, N.M.T., Marcellin, E., et al. (2016) Cyclic-di-AMP synthesis by the diadenylate cyclase CdaA is modulated by the peptidoglycan biosynthesis enzyme GImM in Lactococcus lactis. Mol Microbiol 99: 1015-1027.

Zimmer, B., Hillmann, A., and Görke, B. (2008) Requirements for the phosphorylation of the Escherichia coli EllA Ntr protein in vivo. FEMS Microbiol Lett 286: 96-102.

Zweers, J.C., Wiegert, T., and Dijl, J.M. Van (2009) Stress-responsive systems set specific limits to the overproduction of membrane proteins in Bacillus subtilis. Appl Environ Microbiol 75: 7356-7364. 


\section{Chapter 9 -Supplementary material}

\subsection{Bacterial strains used in this study}

\begin{tabular}{|c|c|c|}
\hline Strain & Genotype & Reference \\
\hline 168 & $\operatorname{trpC2}$ & Laboratory collection \\
\hline BKE14130 & $\operatorname{trpC2} \triangle$ darB::ermC & Koo et al., 2017 \\
\hline BSIP1114 & $\operatorname{trpC2}$ amyE::pta-lacZ cat & $\begin{array}{l}\text { Presecan-Siedel et al., } \\
1999\end{array}$ \\
\hline HB7385 & W168 att SPB trpC2 $\triangle f s r A:: s p c$ & Gaballa et al., 2008 \\
\hline HM1080 & $\operatorname{trpC2} p l s C^{\prime} \Omega\left(P_{x y}-p l s C\right.$ spc) amyE::(Pxyl-xylR tet) & Murray and Koh, 2014 \\
\hline LUW276 & $\operatorname{trpC2}$ amyE::Idh- YacZ cat & Larsson et al., 2005 \\
\hline MDB43 & $\operatorname{trpC2}$ pheA1 yveA::neo & Zaprasis et al., 2015 \\
\hline NCIB3610 & prototrophic, non-domestic WT & Branda et al., 2001 \\
\hline NJ61 & $1 A 100$ attSPB trpC2 amyE::(P $P_{x y l}-a c c D A$ spec $)$ & Jahn et al., 2015 \\
\hline GHB6 & $\operatorname{trpC2}$ pheA1 $\Delta k \operatorname{trC}:: s p c$ & Holtmann et al., 2003 \\
\hline GHB12 & trpC2 pheA1 $\triangle k t r D:: t e t$ & Holtmann et al., 2003 \\
\hline GP92 & $\operatorname{trp} C 2 \triangle k \operatorname{tr} A B:: k a n$ & Gundlach et al., 2017b \\
\hline GP93 & $\operatorname{trpC2} \Delta$ kimA::cat & Gundlach et al., 2017b \\
\hline GP94 & $\operatorname{trpC2} \triangle c d a A:: s p c$ & Gundlach, 2017 \\
\hline GP205 & $\operatorname{trpC2}$ amyE::(citB-`lacZ cat) & Blencke et al., 2006 \\
\hline GP206 & $\operatorname{trpC2}$ amyE::(citB-`lacZ cat) $\triangle c c p C:: k a n$ & Blencke et al., 2006 \\
\hline GP210 & $\operatorname{trpC2}$ amyE::(ccpC- YacZ cat) & Blencke, 2001 \\
\hline GP327 & $\operatorname{trpC2}$ amyE::(ilvA- 'lacZ cat) & Ludwig, 2002 \\
\hline GP330 & $\operatorname{trpC2}$ amyE::(ilvD-`lacZ cat) & Ludwig, 2002 \\
\hline GP342 & $\operatorname{trpC2}$ amyE::(gltA-`lacZ kan) & Wacker et al., 2003 \\
\hline GP706 & $\operatorname{trp} C 2 \Delta g a p A:: e r m C \Delta c c p N:: c a t$ & Pietack, 2010 \\
\hline GP797 & $\operatorname{trpC2} \Delta$ citZ::spec & Klewing, 2013 \\
\hline GP807 & $\operatorname{trp} C 2 \triangle g / t A B:: t e t$ & Gunka, 2010 \\
\hline GP983 & $\operatorname{trpC2} \Delta c d a S:: e r m C$ & Mehne et al., 2013 \\
\hline GP987 & $\operatorname{trp} C 2 \triangle$ disA::tet & Mehne et al., 2013 \\
\hline GP991 & $\operatorname{trp} C 2 \Delta c d a S:: e r m C \Delta$ disA::tet & Mehne et al., 2013 \\
\hline GP997 & $\operatorname{trp} C 2 \triangle c d a A:: c a t$ & Mehne et al., 2013 \\
\hline GP1171 & $\operatorname{trp} C 2 \times k d E::(N-y f p$ ermC) & Gunka, 2010 \\
\hline GP1442 & $\operatorname{trp} C 2 \triangle c c p C:: k a n$ & Meyer, 2012 \\
\hline GP1563 & $\operatorname{trp} C 2 \Delta$ sunA::kan & Jan Gerwig, 2014 \\
\hline GP1712 & $\operatorname{trpC2} \triangle$ darA::cat & Gundlach et al., 2015a \\
\hline GP2030 & $\operatorname{trp} C 2 \Delta k t r D:: t e t$ & Gundlach et al., 2017b \\
\hline GP2032 & $\operatorname{trpC2} \Delta c d a S:: e r m C \Delta c d a A:: c a t$ & Gundlach et al., 2015c \\
\hline GP2048 & $\operatorname{trp} C 2 \Delta k \operatorname{trC}:: c a t$ & Gundlach et al., 2017b \\
\hline GP2079 & $\operatorname{trp} C 2 \Delta k \operatorname{trC}:: t e t$ & Gundlach et al., 2017a \\
\hline GP2136 & $\operatorname{trp} C 2 \Delta k \operatorname{trD}:: t e t \Delta k \operatorname{tr} A B:: k a n$ & Gundlach et al., 2017b \\
\hline GP2165 & $\operatorname{trp} C 2 \Delta k \operatorname{tr} A B:: k a n \Delta k i m A:: c a t$ & Gundlach et al., 2017b \\
\hline
\end{tabular}




\begin{tabular}{|c|c|c|}
\hline Strain & Genotype & Reference \\
\hline GP2166 & $\operatorname{trpC2} \Delta k \operatorname{trC}::$ tet $\Delta k i m A:: c a t$ & Gundlach et al., 2017a \\
\hline GP2167 & $\operatorname{trpC2} \Delta k \operatorname{trD}::$ tet $\Delta k i m A:: c a t$ & Gundlach et al., 2017b \\
\hline GP2169 & $\operatorname{trp} C 2 \Delta k \operatorname{tr} A B:: k a n \Delta k \operatorname{tr} C::$ cat $\Delta k \operatorname{tr} D::$ tet & Gundlach et al., 2017b \\
\hline GP2185 & $\operatorname{trpC2} \Delta a h r C:: e r m C$ & Gundlach et al., 2017a \\
\hline GP2222 & $\operatorname{trpC2} \Delta c d a A:: c a t \Delta c d a S:: e r m C \Delta$ disA::tet & Gundlach et al., 2017b \\
\hline GP2223 & $\operatorname{trpC2} \Delta c d a A:: c a t \Delta c d a S:: e r m C \Delta$ disA::tet nhaK $K_{S 187 F}$ & Gundlach et al., 2017b \\
\hline GP2245 & $\operatorname{trpC2} \Delta c$ daS::ermC $\triangle$ disA::tet $\triangle g \mid t T:: k a n$ & Gundlach, 2017 \\
\hline GP2247 & $\operatorname{trpC2} \Delta g / t T:: e r m C$ & Gundlach, 2017 \\
\hline GP2248 & $\operatorname{trpC2} \Delta g / t T:: k a n$ & Gundlach, 2017 \\
\hline GP2264 & $\operatorname{trpC2} \Delta k \operatorname{trC::kan}$ & Gundlach, 2017 \\
\hline GP2297 & $\operatorname{trpC2} \Delta c d a A:: c a t \Delta c d a S:: s p c$ & Gundlach, 2017 \\
\hline GP2461 & $\operatorname{trpC2} \triangle k \operatorname{tr} A B:: k a n \triangle k i m A::$ cat $\triangle$ darA::spc & Weiß, 2018 \\
\hline GP2473 & $\operatorname{trpC2} \Delta \operatorname{cod} Y:: s p c$ & Weiß, 2018 \\
\hline GP2600 & comIQ12L $\Delta d c t P:: t e t$ & Kampf, 2018 \\
\hline GP2720 & $\operatorname{trpC2} \Delta k \operatorname{kimA}:: s p c$ & Gundlach, 2017 \\
\hline GP2721 & $\operatorname{trpC2} \Delta$ kimA::ermC & Gundlach, 2017 \\
\hline GP2756 & $\operatorname{trpC2} \Delta y d z A-m n t H:: e r m C$ & Gundlach, 2017 \\
\hline GP2811 & $\operatorname{trpC2}$ pGP767 citM( ( cre-site) $_{\text {) }}$ & Krammer, 2017 \\
\hline GP2812 & $\operatorname{trpC2}$ pGP767 citM(cre-site TGTA->TATA) & Krammer, 2017 \\
\hline
\end{tabular}

\subsection{Bacterial strains constructed in this study}

\begin{tabular}{|c|c|c|}
\hline Strain & Genotype & Reference/Construction \\
\hline GP616 & $\operatorname{trpC2} k \operatorname{tr} D^{G 282 V}$ & Krüger et al., 2020b \\
\hline GP617 & $\operatorname{trpC2} k \operatorname{tr} D^{G 282 v} \Delta k \operatorname{trAB}:: s p e c$ & Krüger et al., 2020b \\
\hline GP618 & $\operatorname{trpC2} k \operatorname{tr} D^{G 282 V} \Delta k \operatorname{tr} A B:: s p e c \Delta k i m A:: e r m C$ & Krüger et al., 2020b \\
\hline GP619 & $\operatorname{trpC2} k \operatorname{tr} D^{G 282 v} \Delta k \operatorname{tr} A B::$ spec $\Delta k i m A:: e r m C \Delta k \operatorname{tr} C:: c a t$ & Krüger et al., 2020b \\
\hline GP1054 & $\operatorname{trpC2} \Delta k \operatorname{trC}:: s p e c$ & Krüger et al., 2020b \\
\hline GP1055 & $\operatorname{trpC2} \Delta k \operatorname{trAB}:: k a n \Delta k i m A::$ cat $\Delta k \operatorname{trD}::$ tet & Krüger et al., 2020b \\
\hline GP1088 & $\operatorname{trpC2} \Delta k \operatorname{trAB}:: k a n \Delta k i m A::$ cat $\Delta k \operatorname{tr} D::$ tet $\Delta k \operatorname{trC}:: s p e c$ & Krüger et al., 2020b \\
\hline GP1360 & $\operatorname{trpC2} \Delta c d a S:: s p c$ & Krüger et al., 2020a \\
\hline GP1361 & $\operatorname{trpC2} \triangle c d a S:: s p c \operatorname{dis} A:: t e t$ & Krüger et al., 2020a \\
\hline GP2168 & $\operatorname{trpC2} \Delta k \operatorname{trC}::$ cat $\Delta k \operatorname{trD}::$ tet & Krüger et al., 2020b \\
\hline GP2181 & $\operatorname{trpC2}$ amyE::(P-kimA-RS-lacZ cat) & Krüger et al., 2020a \\
\hline GP2224 & $\begin{array}{l}\operatorname{trpC2} \Delta c d a A:: c a t \Delta c d a S:: e r m C \Delta \text { disA::tet aimA } \text { frameshift } \\
\text { yqeG }_{\text {frameshift }} \text { yqeV } \\
\text { frameshift }\end{array}$ & Krüger et al., 2020a \\
\hline GP2245 & $\operatorname{trpC2} \Delta c$ daS::ermC $\triangle$ disA::tet $\Delta g \mid t T:: k a n$ & Krüger et al., 2020a \\
\hline GP2248 & $\operatorname{trpC2} \Delta g / t T:: k a n$ & Krüger et al., 2020a \\
\hline GP2259 & $\operatorname{trpC2} \triangle c d a A::$ cat $\Delta c d a S:: e r m C \Delta g / t T:: k a n \Delta d i s A::$ tet & Krüger et al., 2020a \\
\hline GP2413 & $\operatorname{trpC2} \Delta c$ daS::ermC $\triangle$ disA::tet $\triangle$ darA::cat & Krüger et al., 2020a \\
\hline
\end{tabular}




\begin{tabular}{|c|c|c|}
\hline Strain & Genotype & Reference/Construction \\
\hline GP2415 & $\operatorname{trpC2} \triangle d a r A:: s p c$ & Krüger et al., 2020b \\
\hline GP2420 & $\operatorname{trpC2} \Delta c$ daS::ermC $\triangle$ disA::tet $\triangle$ darA::cat $\triangle c d a A:: s p c$ & Krüger et al., 2020a \\
\hline GP2461 & 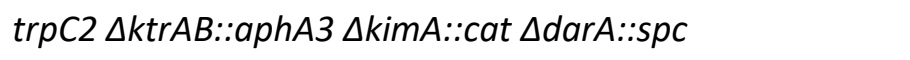 & Krüger et al., 2020b \\
\hline GP2495 & $\operatorname{trpC2} \Delta k \operatorname{tr} A B:: a p h A 3 \Delta k i m A::$ cat $\triangle$ darA::spc $\Delta a h r C:: e r m C$ & Krüger et al., 2020b \\
\hline GP2716 & $\operatorname{trp} C 2 \Delta k \operatorname{tr} A B:: s p c$ & Krüger et al., 2020b \\
\hline GP2721 & $\operatorname{trpC2} \Delta$ kimA::ermC & Krüger et al., 2020b \\
\hline GP2760 & $\operatorname{trpC2} \Delta$ citM::kan & $\mathrm{LFH} \rightarrow 168$ \\
\hline GP2761 & $\operatorname{trpC2} \Delta$ citM::spec & $\mathrm{LFH} \rightarrow 168$ \\
\hline GP2762 & $\operatorname{trpC2} \Delta m g t E:: s p e c$ & $\mathrm{LFH} \rightarrow 168$ \\
\hline GP2763 & $\operatorname{trpC2} \Delta$ citM::kan $\Delta m g t E:: s p e c$ & $\mathrm{LFH} \rightarrow \mathrm{GP} 2760$ \\
\hline GP2764 & $\operatorname{trpC2}$ amyE::(citM- YacZ cat) & pGP2965 $\rightarrow 168$ \\
\hline GP2765 & $\operatorname{trpC2}$ amyE::(citM( $\Delta$ cre-site)- 'lacZ cat) & pGP2966 $\rightarrow 168$ \\
\hline GP2766 & $\operatorname{trpC2}$ amyE::(citM (cre-site TGTA->TATA)- `lacZ cat) & pGP2967 $\rightarrow 168$ \\
\hline GP2767 & $\operatorname{trpC2}$ citM-3x FLAG spec & pGP2968 $\rightarrow 168$ \\
\hline GP2768 & $\operatorname{trpC2}$ mgtE-3x FLAG spec & pGP2969 $\rightarrow 168$ \\
\hline GP2769 & $\operatorname{trpC2} \triangle$ darB::ermC & BKE14130 $\rightarrow 168$ \\
\hline GP2770 & $\operatorname{trpC2} \Delta$ citM::kan pGP1550 (darB $\left.{ }^{+}\right)$odh $A_{f s}$ yoa $E_{H 188 Q} y c b P_{f s}$ & $\begin{array}{l}\text { pGP1550 } \rightarrow \text { GP2760, } \\
\text { suppressor mutant }\end{array}$ \\
\hline GP2771 & $\operatorname{trpC2} \Delta k \operatorname{trC}:: s p c$ & $\mathrm{LFH} \rightarrow 168$ \\
\hline GP2772 & $\operatorname{trpC2} \Delta c d a A:: c a t \Delta c d a S:: s p c \Delta y f j Q::$ tet & GP2853 $\rightarrow$ GP2297 \\
\hline GP2773 & $\operatorname{trpC2} \Delta$ kimA::cat $\Delta k \operatorname{tr} D::$ tet & GP2030 $\rightarrow$ GP93 \\
\hline GP2774 & $\operatorname{trpC2} \Delta c d a A:: c a t \Delta c d a S:: e r m C \Delta$ disA::tet $k \operatorname{tr} B_{M 1 A} f_{l i Y} Y_{f s}$ & Suppressor of GP2222 \\
\hline GP2775 & $\operatorname{trpC2} \Delta$ kimA::cat $\Delta k \operatorname{tr} D::$ tet $\Delta k \operatorname{tr} A:: k a n$ & GP3065 $\rightarrow$ GP2773 \\
\hline GP2776 & $\operatorname{trpC2} \triangle$ darB::ermC yer $D_{\text {bp1131G }>A \text {, silent }}$ & Suppressor of GP2769 \\
\hline GP2777 & $\operatorname{trpC2} \Delta c$ daS::spc $\triangle$ disA::tet $\Delta$ darB::ermC & Krüger et al., 2020c \\
\hline GP2778 & $\operatorname{trpC2} \Delta c$ daS::spc $\Delta$ disA::tet $\Delta m p r F:: e r m C$ & GP2246 $\rightarrow$ GP1361 \\
\hline GP2779 & $\operatorname{trpC2} \Delta c$ daS::spc $\triangle$ disA::tet $\triangle$ darB::ermC $\triangle c d a A:: c a t$ & Krüger et al., 2020a \\
\hline GP2780 & $\operatorname{trpC2} \Delta c$ daS::spc $\triangle$ disA::tet $\Delta m p r F: e r m C \Delta c d a A::$ cat & GP997 $\rightarrow$ GP2778 \\
\hline GP2781 & $\operatorname{trpC2} \triangle$ darB::ermC $\triangle$ darA::cat & BKE14130 $\rightarrow$ GP1712 \\
\hline GP2782 & $\operatorname{trpC2} \triangle \operatorname{dis} A:: k a n$ & $\mathrm{LFH} \rightarrow 168$ \\
\hline GP2783 & $\operatorname{trpC2} \Delta k \operatorname{trC}::$ cat $\Delta k \operatorname{tr} D::$ tet $\Delta k i m A::$ ermC & Krüger et al., 2020b \\
\hline GP2784 & $\operatorname{trpC2} \Delta c$ daS::spc $\Delta$ disA::tet $\Delta m p r F: e r m C \Delta y d g F-y d g J$ & Suppressor of GP2778 \\
\hline GP2785 & $\operatorname{trpC2} \Delta y f k C:: k a n$ & Krüger et al., 2020a \\
\hline GP2786 & $\operatorname{trpC2} \Delta$ aimA::kan & Krüger et al., 2020a \\
\hline GP2787 & $\operatorname{trpC2} \Delta$ kimA::cat $\Delta k \operatorname{trD}::$ tet $\Delta k \operatorname{trC}:: s p c$ & GP2771 $\rightarrow$ GP2773 \\
\hline GP2788 & $\operatorname{trpC2} \mathrm{mprF}_{\mathrm{H} 489 \mathrm{Q}} \mathrm{kan}$ & $\mathrm{LFH} \rightarrow 168$ \\
\hline GP2789 & $\operatorname{trpC2} \Delta g / t T:: k a n \Delta d c t P:: t e t$ & GP2600 $\rightarrow$ GP2248 \\
\hline GP2790 & $\operatorname{trpC2} \triangle c d a A:: k a n$ & LFH $\rightarrow 168$ \\
\hline GP2791 & $\operatorname{trpC2} \Delta c$ daS::spc $\Delta$ disA::tet $\Delta y k u L:: e r m C \Delta$ darA::cat & GP1712 $\rightarrow$ GP2777 \\
\hline
\end{tabular}




\begin{tabular}{|c|c|c|}
\hline Strain & Genotype & Reference/Construction \\
\hline GP2792 & $\begin{array}{l}\operatorname{trpC2} \triangle c d a S:: s p c \triangle d i s A:: t e t \Delta y k u L:: e r m C \triangle d a r A:: c a t \\
\Delta c d a A:: k a n\end{array}$ & GP2790 $\rightarrow$ GP2791 \\
\hline GP2793 & $\operatorname{trpC2} \triangle c$ cdaS::spc disA::tet mprF ${ }_{H 489 Q} k a n$ & GP2788 $\rightarrow$ GP1361 \\
\hline GP2794 & $\operatorname{trpC2} \Delta k i m A::$ cat $\Delta k \operatorname{trD}::$ tet $\Delta k \operatorname{tr} A:: k a n \Delta k \operatorname{trC}:: s p c$ & GP2771 $\rightarrow$ GP2775 \\
\hline GP2795 & $\operatorname{trpC2} \Delta y v e A:: s p e c$ & $\mathrm{LFH} \rightarrow 168$ \\
\hline GP2796 & $\operatorname{trpC2} \Delta c d a S:: s p c$ disA::tet $\triangle y f k C:: k a n$ & Krüger et al., 2020a \\
\hline GP2797 & $\operatorname{trpC2} \Delta c d a S:: s p c$ disA::tet $\Delta y f k C:: k a n \triangle c d a A:: c a t$ & Krüger et al., 2020a \\
\hline GP2798 & $\operatorname{trpC2} y f k C_{R 42 W}$ kan & Krüger et al., 2020a \\
\hline GP2799 & $\operatorname{trpC2} \Delta g / t P:: k a n$ & LFH $\rightarrow 168$ \\
\hline GP2800 & $\operatorname{trpC2} \triangle$ darB-ccpC::phleo & LFH $\rightarrow 168$ \\
\hline GP2813 & $\operatorname{trpC2} \triangle c$ daS::spc disA::tet $y f k C_{R 42 W}$ kan & Krüger et al., 2020a \\
\hline GP2814 & $\operatorname{trpC2} \Delta c$ daS::spc disA::tet yfkC $C_{R 42 w}$ kan $\triangle c d a A:: c a t$ & Krüger et al., 2020a \\
\hline GP2815 & $\operatorname{trpC2} \Delta c$ daS::ermC $\triangle$ disA::tet $\Delta g / t T:: k a n \Delta k i m A:: c a t$ & GP93 $\rightarrow$ GP2245 \\
\hline GP2816 & $\begin{array}{l}\operatorname{trpC2} \triangle c d a S:: \text { ermC } \triangle \text { disA::tet } \triangle g l t T:: k a n \Delta k i m A:: c a t \\
\triangle c d a A:: s p c\end{array}$ & GP94 $\rightarrow$ GP2815 \\
\hline GP2817 & $\operatorname{trp} C 2 \Delta c d a S:: e r m C \Delta$ disA::tet $\triangle k \operatorname{tr} A B:: k a n$ & GP92 $\rightarrow$ GP991 \\
\hline GP2818 & $\operatorname{trpC2} \Delta g l t P:: c a t \Delta a i m A:: k a n$ & Krüger et al., 2020a \\
\hline GP2819 & $\operatorname{trpC2} \Delta c d a S:: s p c$ disA::tet $\Delta g / t T:: e r m C$ & GP2247 $\rightarrow$ GP1361 \\
\hline GP2820 & $\operatorname{trpC2} \triangle g / t P::$ cat $\triangle y b e C:: k a n \Delta g l t A B::$ tet & GP807 $\rightarrow$ GP2818 \\
\hline GP2821 & $\operatorname{trpC2} y f k C_{w T} k a n$ & $\mathrm{LFH} \rightarrow 168$ \\
\hline GP2822 & $\operatorname{trpC2} \triangle y v e A:: n e o$ & MDB43 $\rightarrow 168$ \\
\hline GP2823 & $\operatorname{trpC2}$ amyE::(ccpC- lacZ cat) $\triangle c c p C:: k a n$ & GP210 $\rightarrow$ GP1445 \\
\hline GP2824 & $\operatorname{trpC2} \Delta g / t P:: c a t$ & Krüger et al., 2020a \\
\hline GP2825 & $\operatorname{trpC2} \Delta g / t T:: k a n \Delta g l t P:: c a t$ & Krüger et al., 2020a \\
\hline GP2826 & $\operatorname{trpC2} \triangle g \mid t T:: k a n \Delta g / t P:: c a t \Delta g l t A B:: t e t$ & GP807 $\rightarrow$ GP2825 \\
\hline GP2827 & $\operatorname{trpC2} \Delta g \mid t T:: k a n \Delta g l t P:: c a t \Delta g l t A B::$ tet $\triangle$ kimA::spec & GP2720 $\rightarrow$ GP2826 \\
\hline GP2828 & $\operatorname{trpC2} \Delta g \mid t T:: k a n \Delta g l t P::$ cat $\triangle g l t A B::$ tet $\triangle$ kimA::ermC & GP2721 $\rightarrow$ GP2826 \\
\hline GP2829 & $\operatorname{trpC2} \Delta g l t T:: k a n \Delta g l t P::$ cat $\triangle g \mid t A B::$ tet $\triangle y v e A:: s p e c$ & GP2795 $\rightarrow$ GP2826 \\
\hline GP2830 & $\begin{array}{l}\operatorname{trpC2} \Delta g l t T:: k a n \triangle g l t P:: c a t \Delta g l t A B:: \text { tet } \triangle \text { kimA::ermC } \\
\triangle y v e A:: \text { spec }\end{array}$ & GP2795 $\rightarrow$ GP2828 \\
\hline GP2831 & $\operatorname{trpC2} \Delta g / t T:: k a n \Delta a i m A:: p h l e o$ & Krüger et al., 2020a \\
\hline GP2832 & $\begin{array}{l}\operatorname{trpC2} \triangle g l t T:: k a n \triangle g l t P:: \text { cat } \triangle g l t A B:: \text { tet } \triangle y v e A:: s p e c \\
\Delta y b e C:: \text { phleo }\end{array}$ & GP2831 $\rightarrow$ GP2829 \\
\hline GP2833 & $\begin{array}{l}\operatorname{trpC2} \Delta g l t T:: k a n \Delta g l t P:: c a t \Delta g l t A B:: \text { tet } \triangle k i m A:: e r m C \\
\Delta y v e A:: s p e c \Delta y b e C:: \text { phleo }\end{array}$ & GP2831 $\rightarrow$ GP2830 \\
\hline GP2834 & $\operatorname{trpC2}$ gudB $B^{++}$ & 168 , selection on $\mathrm{CE}$ \\
\hline GP2835 & $\operatorname{trpC2} \Delta g / t T:: k a n \triangle g l t P:: c a t \Delta g l t A B::$ tet $\triangle y b e C:: p h l e o$ & GP2831 $\rightarrow$ GP2826 \\
\hline GP2836 & $\begin{array}{l}\text { trpC2 } \Delta g l t T:: k a n \triangle g l t P:: \text { cat } \triangle g l t A B:: \text { tet } \triangle \text { kimA::ermC } \\
\triangle y b e C:: \text { phleo }\end{array}$ & GP2831 $\rightarrow$ GP2828 \\
\hline
\end{tabular}




\begin{tabular}{|c|c|c|}
\hline Strain & Genotype & Reference/Construction \\
\hline GP2837 & 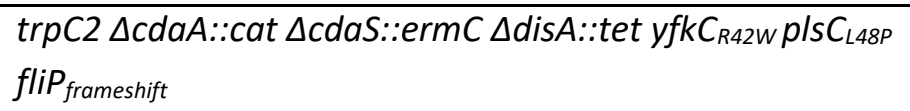 & Krüger et al., 2020a \\
\hline GP2838 & 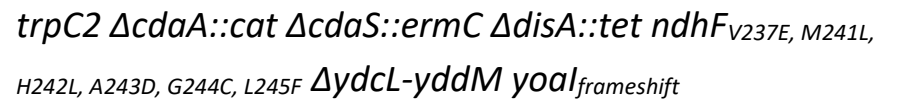 & Krüger et al., 2020a \\
\hline GP2839 & $\begin{array}{l}\operatorname{trpC2} \Delta c d a A:: c a t \Delta c d a S:: e r m C \Delta d i s A:: \text { tet flik } K_{\text {truncated }} \\
\operatorname{rpoB}_{Q 109 p} r b s B_{R 175 L}\end{array}$ & Krüger et al., 2020a \\
\hline GP2840 & $\begin{array}{l}\operatorname{trpC2} \Delta c d a A:: c a t \Delta c d a S:: e r m C \Delta d i s A:: t e t p l s C^{A 61 V} n d h F^{\mathrm{FS}} \\
k \operatorname{trC} C^{\mathrm{FS}} \operatorname{sig} D^{G 88 D}\end{array}$ & Krüger et al., 2020a \\
\hline GP2841 & $\begin{array}{l}\operatorname{trpC2} \triangle c d a A:: \text { cat } \triangle c d a S:: e r m C \triangle d i s A:: t e t \text { gudB } B_{\text {decrypt. }} \\
\operatorname{accC}_{\mathrm{H} 298 \mathrm{Y}}\end{array}$ & Krüger et al., 2020a \\
\hline GP2842 & $\begin{array}{l}\operatorname{trp} C 2 \Delta c d a A:: c a t \Delta c d a S:: e r m C \Delta d i s A:: \text { tet } n h a K_{\mathrm{S} 187 \mathrm{~F}} \\
\operatorname{aimA}_{\text {frameshift }}\end{array}$ & Krüger et al., 2020a \\
\hline GP2843 & $\begin{array}{l}\operatorname{trpC2} \Delta c d a S:: s p c \Delta d i s A:: \text { tet } \Delta \text { darB::ermC } \Delta c d a A:: \text { cat } \\
\text { ybeC }_{\text {frameshift }} \text { hprK } \\
\text { A132T }\end{array}$ & Suppressor of GP2779 \\
\hline GP2844 & $\begin{array}{l}\operatorname{trpC2} \Delta c d a S:: s p c \Delta d i s A:: \text { tet } \triangle \text { darB::ermC } \Delta c d a A:: \text { cat } \\
k \operatorname{ktC} C_{\text {truncation }}\end{array}$ & Suppressor of GP2779 \\
\hline GP2845 & $\begin{array}{l}\operatorname{trpC2} \Delta c d a S:: s p c \Delta \text { disA::tet } \Delta \text { darB::ermC } \Delta c d a A:: \text { cat } \\
k_{\operatorname{trC}} \text { frameshift } \text { kimA }_{\triangle 354-364} \text { darA } A_{V 100 A}\end{array}$ & Krüger et al., 2020a \\
\hline GP2846 & $\begin{array}{l}\operatorname{trpC2} \Delta c d a S:: s p c \triangle d i s A:: \text { tet } \triangle \text { darB::ermC } \Delta c d a A:: \text { cat } \\
\text { opuAB }_{A 262 V}\end{array}$ & Suppressor of GP2779 \\
\hline GP2847 & $\begin{array}{l}\operatorname{trpC2} \Delta c d a S:: s p c \Delta \text { disA::tet } \Delta \text { darB::ermC } \Delta c d a A:: \text { cat } \\
\text { opuAB }_{\text {A262v }} a_{c c} A_{\text {frameshift }} k t r B_{\text {frameshift }} m m_{\text {A401v }} y m c B_{\text {frameshift }}\end{array}$ & Suppressor of GP2846 \\
\hline GP2848 & $\begin{array}{l}\operatorname{trpC2} \Delta c d a S:: s p c \triangle d i s A:: \text { tet } \triangle \text { darB::ermC } \triangle c d a A:: \text { cat } \\
\text { aslA } A_{A 8 s v}\end{array}$ & Suppressor of GP2779 \\
\hline GP2849 & $\begin{array}{l}\operatorname{trpC2} \triangle c d a A:: c a t \Delta c d a S:: e r m C \Delta \text { disA::tet aimA } A_{\text {frameshift }} \\
y b c C_{K 722 L / 1724 F}\end{array}$ & Krüger et al., 2020a \\
\hline GP2850 & $\begin{array}{l}\operatorname{trpC2} \Delta c d a S:: s p c \text { disA::tet yfk } C_{R 42 W} \text { kan } \triangle c d a A:: c a t \\
a_{c c A_{A 229 V}} \text { yerC } C_{\text {T58K }} \text { ypo } P_{\text {KTEYF } 1111}\end{array}$ & Krüger et al., 2020a \\
\hline GP3010 & 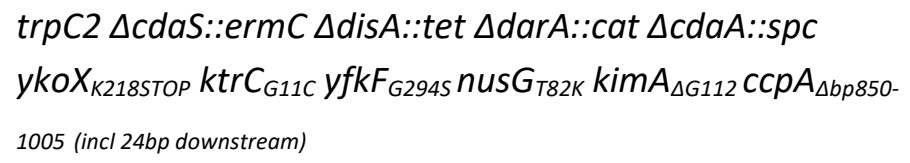 & Krüger et al., 2020a \\
\hline GP3051 & $\operatorname{trp} C 2 \Delta c d a S::$ ermC $\triangle$ disA::tet $\Delta k \operatorname{trC}:: k a n$ & GP2264 $\rightarrow$ GP991 \\
\hline GP3052 & $\operatorname{trpC2} \Delta k \operatorname{tr} A B:: k a n \triangle k i m A:: c a t \Delta$ darB::ermC & BKE14130 $\rightarrow$ GP2165 \\
\hline GP3053 & $\operatorname{trpC2} \Delta c$ daS::ermC $\triangle$ disA::tet $\triangle$ aimA::phleo & Krüger et al., 2020a \\
\hline GP3054 & $\operatorname{trp} C 2 \Delta c d a S::$ ermC $\triangle$ disA::tet $\triangle$ aimA::phleo $\triangle c d a A::$ cat & Krüger et al., 2020a \\
\hline GP3055 & $\operatorname{trpC2} \Delta k \operatorname{trC}::$ cat $\Delta k \operatorname{trD}::$ tet $\Delta k i m A:: e r m C \Delta k \operatorname{trA}:: k a n$ & Krüger et al., 2020b \\
\hline GP3056 & $\operatorname{trp} C 2 \Delta k \operatorname{tr} A B:: k a n \Delta k i m A::$ cat $\Delta k \operatorname{trC}::$ tet & Krüger et al., 2020b \\
\hline GP3057 & $\operatorname{trpC2} \Delta c d a S:: e r m C \Delta$ disA::tet $\Delta$ darB-ccpC::phleo & GP2800 $\rightarrow$ GP991 \\
\hline GP3058 & $\begin{array}{l}\operatorname{trpC2} \Delta c d a S:: e r m C \triangle d i s A:: t e t \triangle d a r B-c c p C:: p h l e o \\
\Delta c d a A:: c a t\end{array}$ & GP997 $\rightarrow$ GP3057 \\
\hline GP3059 & $\operatorname{trp} C 2$ gudB $B^{++} \Delta c d a S:: e r m C$ & GP983 $\rightarrow$ GP2834 \\
\hline GP3060 & $\operatorname{trpC2}$ gudB ${ }^{++} \Delta c d a S:: e r m C \Delta$ disA::tet & GP987 $\rightarrow$ GP3059 \\
\hline
\end{tabular}




\begin{tabular}{|c|c|c|}
\hline Strain & Genotype & Reference/Construction \\
\hline GP3061 & $\operatorname{trpC2} \triangle c c p C:: k a n$ pGP3306 (darB $\left.{ }^{+}\right)$spoOB $B_{D 3 E}$ & $\begin{array}{l}\text { pGP3306 } \rightarrow \text { GP206, } \\
\text { Suppressor mutant }\end{array}$ \\
\hline GP3062 & $\operatorname{trpC2}$ ybec-3x FLAG spec & pGP2990 $\rightarrow 168$ \\
\hline GP3063 & $\operatorname{trpC2}$ gltT-3x FLAG spec & pGP2991 $\rightarrow 168$ \\
\hline GP3064 & $\operatorname{trpC2} \Delta k \operatorname{trB}:: k a n$ & Krüger et al., 2020b \\
\hline GP3065 & $\operatorname{trpC2} \Delta k \operatorname{trA}:: k a n$ & Krüger et al., 2020b \\
\hline GP3066 & $\operatorname{trpC2} \Delta$ ktrAB::kan $\triangle$ kimA::cat $\triangle$ darB::ermC $\triangle a h r C:: p h l e o$ & GP3122 $\rightarrow$ GP3052 \\
\hline GP3067 & $\operatorname{trpC2} \Delta c d a S:: s p c \Delta d i s A::$ tet $\Delta$ darB::ermC $\Delta k \operatorname{trC}:: k a n$ & GP2264 $\rightarrow$ GP2777 \\
\hline GP3068 & $\begin{array}{l}\operatorname{trpC2} \Delta c d a S:: s p c \Delta d i s A:: \text { tet } \triangle \text { darB::ermC } \Delta k \operatorname{trC}:: k a n \\
\Delta c d a A:: c a t\end{array}$ & GP997 $\rightarrow$ GP3067 \\
\hline GP3069 & $\operatorname{trpC2} \Delta c$ daS::spc $\Delta$ disA::tet $\triangle$ darB::ermC $\Delta$ aimA::phleo & GP2831 $\rightarrow$ GP2777 \\
\hline GP3070 & $\begin{array}{l}\operatorname{trpC2} \Delta c \text { daS::spc } \triangle \text { disA::tet } \triangle \text { darB::ermC } \triangle \text { aimA::phleo } \\
\Delta c d a A:: c a t\end{array}$ & GP997 $\rightarrow$ GP3069 \\
\hline GP3071 & $\operatorname{trpC2} \Delta g / t T:$ kan $\triangle$ aimA::phleo $\Delta g l t P:: c a t$ & Krüger et al., 2020a \\
\hline GP3072 & $\operatorname{trpC2} \triangle$ aimA::kan $\triangle c d a S:: e r m C$ & GP983 $\rightarrow$ GP2876 \\
\hline GP3073 & $\operatorname{trpC2} \Delta$ aimA::kan $\Delta c d a S:: s p c$ & GP1360 $\rightarrow$ GP2876 \\
\hline GP3074 & $\operatorname{trpC2} \triangle$ aimA::kan $\triangle$ darB::ermC & GP2769 $\rightarrow$ GP2876 \\
\hline GP3075 & $\operatorname{trpC2} \Delta$ aimA::kan $\Delta c$ daS::ermC $\triangle$ disA::tet & GP987 $\rightarrow$ GP3072 \\
\hline GP3076 & $\operatorname{trpC2} \Delta$ aimA::kan $\triangle$ darB::ermC $\Delta c d a S:: s p c$ & GP1360 $\rightarrow$ GP3074 \\
\hline GP3077 & $\begin{array}{l}\operatorname{trpC2} \Delta c d a A:: c a t \Delta c d a S:: e r m C \triangle d i s A:: \text { tet } k \operatorname{kt} C_{\text {frameshift }} \\
\operatorname{aimA} / g \mid p Q_{\text {truncation }} y b c C_{\text {frameshift }}\end{array}$ & Krüger et al., 2020a \\
\hline GP3078 & $\begin{array}{l}\operatorname{trpC2} \triangle c d a A:: c a t \Delta c d a S:: e r m C \Delta \text { disA::tet pls } C_{A 61 V} \\
\operatorname{aimA}_{T 374 M} k \operatorname{tr} B_{G 313 D} \operatorname{sig} D_{G 88 D}\end{array}$ & Krüger et al., 2020a \\
\hline GP3079 & $\begin{array}{l}\operatorname{trpC2} \Delta c d a A:: c a t \Delta c d a S:: e r m C \Delta d i s A:: \text { tet } y f k C_{R 42 W} p l s C_{L 48 P} \\
\Delta(a b h-k i n C-y k q A-k \operatorname{trC}) h b s_{132 T} n d o A_{G 65} f_{l i P_{\text {frameshift }}}\end{array}$ & Krüger et al., 2020a \\
\hline GP3080 & $\operatorname{trpC2} x k d E::\left(P_{x y l} \operatorname{aim} A\right.$ erm $\left.C\right)$ & pGP2980 $\rightarrow 168$ \\
\hline GP3081 & $\operatorname{trpC2}$ amyE::(citB- 'lacZ cat) $\triangle c c p C:: k a n \triangle d a r B:: e r m C$ & GP2769 $\rightarrow$ GP206 \\
\hline GP3082 & $\operatorname{trpC2} \Delta k \operatorname{trD}::$ tet $\triangle$ kimA::cat $\Delta k \operatorname{trA}:: k a n$ & Krüger et al. 2020b \\
\hline GP3083 & $\operatorname{trpC2} \Delta c d a S:: e r m C \Delta$ disA::tet $\Delta$ kimA::spec & GP2720 $\rightarrow$ GP991 \\
\hline GP3084 & $\operatorname{trpC2} \Delta c d a S::$ ermC $\Delta$ disA::tet $\Delta k \operatorname{trC}:: k a n \Delta k i m A:: s p e c$ & GP2720 $\rightarrow$ GP3051 \\
\hline GP3085 & $\operatorname{trpC2}$ ganA::(gdhA $\left.A_{E c o}\right):: k a n$ & pGP3000 $\rightarrow 168$ \\
\hline GP3086 & $\operatorname{trpC2} \triangle$ darA::cat ganA:::(gdhA $\left.A_{E c o}\right):: k a n$ & pGP3000 $\rightarrow$ GP1712 \\
\hline GP3087 & $\operatorname{trpC2} \triangle$ darA::cat ganA::(gdhA $\left.A_{E c o}\right):: k a n \triangle g \mid t A B::$ tet & GP807 $\rightarrow$ GP3086 \\
\hline GP3088 & $\operatorname{trpC2} \triangle c c p C::$ kan pGP3306 $\left(\right.$ darB $\left.^{+}\right)$odh $A_{A 697 V}$ purK $_{A 171 T}$ & $\begin{array}{l}\text { pGP3306 } \rightarrow \text { GP206, } \\
\text { suppressor mutant }\end{array}$ \\
\hline GP3089 & $\operatorname{trpC2} \Delta c$ daS::spc $\triangle$ disA::tet amyE::(aimA-lacZ cat) & pGP2287 $\rightarrow$ GP1361 \\
\hline GP3090 & $\begin{array}{l}\operatorname{trpC2} \Delta c d a S:: s p c \triangle d i s A:: \text { tet amyE::(aimA-lacZ cat) } \\
\triangle c d a A:: k a n\end{array}$ & GP2790 $\rightarrow$ GP3089 \\
\hline GP3091 & $\operatorname{trpC2} \Delta c d a S:: s p c$ gudB $B^{++}$ & GP1360 $\rightarrow$ GP2834 \\
\hline GP3092 & $\operatorname{trpC2} \Delta c$ daS::spc gudB ${ }^{++} \Delta$ disA::tet & GP987 $\rightarrow$ GP3091 \\
\hline
\end{tabular}




\begin{tabular}{|c|c|c|}
\hline Strain & Genotype & Reference/Construction \\
\hline GP3093 & 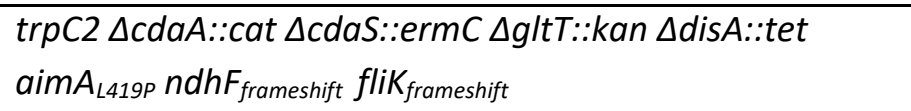 & Krüger et al., 2020a \\
\hline GP3094 & $\begin{array}{l}\operatorname{trpC2} \Delta c \text { daS::spc } \Delta \text { disA::tet } \Delta \text { darB::ermC } \Delta c d a A:: c a t \\
k_{\operatorname{tr} C_{\text {frameshift }}} \text { kimA }_{\triangle 354-364} \operatorname{cod} Y_{G 146 V} \text { yon } D_{D 255 H}\end{array}$ & Krüger et al., 2020a \\
\hline GP3095 & $\operatorname{trpC2} \Delta c$ daS::ermC $\triangle$ disA::tet $\triangle$ aimA::phleo $\Delta g / t T:: k a n$ & GP2248 $\rightarrow$ GP3053 \\
\hline GP3096 & $\begin{array}{l}\operatorname{trpC2} \Delta c d a S:: \text { ermC } \triangle \text { disA::tet } \triangle \text { aimA::phleo } \Delta \text { gltT::kan } \\
\Delta c d a A:: c a t\end{array}$ & GP997 $\rightarrow$ GP3095 \\
\hline GP3097 & $\begin{array}{l}\operatorname{trpC2} \Delta g / t T:: k a n \Delta g l t P:: c a t \Delta g l t A B:: \text { tet } \triangle \text { aimA::phleo } \\
x k d E::\left(P_{x y l} \operatorname{aimA} \text { ermC) }\right.\end{array}$ & GP3080 $\rightarrow$ GP2835 \\
\hline GP3098 & $\begin{array}{l}\operatorname{trpC2} \Delta g / t T:: k a n \Delta g l t P:: \text { cat gltAB::tet } \triangle a i m A:: p h l e o \\
x k d E::\left(P_{x y l} N \text {-yfp ermC) }\right.\end{array}$ & GP1171 $\rightarrow$ GP2835 \\
\hline GP3099 & $\begin{array}{l}\operatorname{trpC2} \Delta c d a A:: c a t \Delta c d a S:: e r m C \Delta d i s A:: t e t p l s C_{L 48 P} y f k C_{R 42 W} \\
\text { fliP }_{\text {frameshift }} \cot O_{134 F}\end{array}$ & Krüger et al., 2020a \\
\hline GP3100 & $\begin{array}{l}\operatorname{trpC2} \Delta k \operatorname{tr} A B:: a p h A 3 \Delta k i m A:: c a t \Delta d a r A:: s p c \Delta a h r C:: e r m C \\
k \operatorname{tr} D^{G 282 v}\end{array}$ & Krüger et al., 2020b \\
\hline GP3101 & $\begin{array}{l}\text { trpC2 } \triangle c \text { daS::ermC } \triangle \text { disA::tet } \triangle \text { aimA::phleo } \Delta c \text { daA::cat } \\
\text { fusA } A_{A 579 v} \text { yqe } G_{\text {frameshift }}\end{array}$ & Krüger et al., 2020a \\
\hline GP3102 & 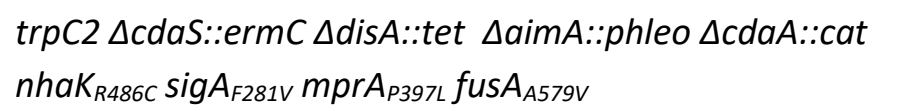 & Krüger et al., 2020a \\
\hline GP3103 & $\begin{array}{l}\operatorname{trpC2} \Delta c \text { daS::ermC } \triangle \text { disA::tet } \triangle \text { aimA::phleo } \Delta c d a A:: c a t \\
\operatorname{ktr}_{G 14 D} \text { top }_{K 13 N} f u s A_{A 21 T}\end{array}$ & Krüger et al., 2020a \\
\hline GP3104 & $\operatorname{trpC2}$ lacA::( $P_{\text {deqQ }}$ darB aphA3) & pGP3308 $\rightarrow 168$ \\
\hline GP3105 & 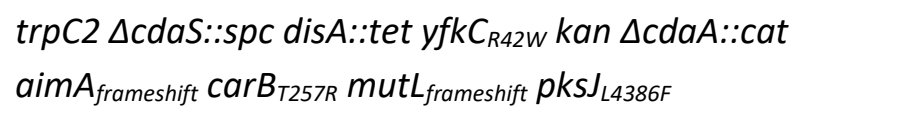 & Krüger et al., 2020a \\
\hline GP3106 & $\operatorname{trpC2}$ accA $_{A 229 \mathrm{~V}}$ phleo & $\mathrm{LFH} \rightarrow 168$ \\
\hline GP3107 & $\operatorname{trpC2}$ acc $_{w T}$ phleo & $\mathrm{LFH} \rightarrow 168$ \\
\hline GP3108 & $\operatorname{trpC2}$ acc $_{\mathrm{H} 298 \mathrm{r}}$ phleo & $\mathrm{LFH} \rightarrow 168$ \\
\hline GP3109 & $\operatorname{trpC2} \operatorname{acc}_{W T}$ phleo & $\mathrm{LFH} \rightarrow 168$ \\
\hline GP3110 & $\operatorname{trpC2} \Delta c d a S:: s p c$ disA::tet accA $A_{A 229 v}$ phleo & GP3106 $\rightarrow$ GP1361 \\
\hline GP3111 & $\operatorname{trpC2} p l s C^{\prime} \Omega\left(P_{x y}-p / s C\right.$ spc) amyE::(Pxyl-xylR tet) & $\mathrm{HM} 1080 \rightarrow 168$ \\
\hline GP3112 & $\begin{array}{l}\operatorname{trpC2} \Delta c d a S:: e r m C \Delta c d a A:: c a t \text { pls } C^{\prime} \Omega\left(P_{x y}-p l s C \text { spc }\right) \\
\text { amyE::(Pyyl-xylR tet })\end{array}$ & HM1080 $\rightarrow$ GP2032 \\
\hline GP3113 & $\operatorname{trpC2}$ amyE::(P $P_{x y l}-a c c D A$ spec $)$ & NJ61 $\rightarrow 168$ \\
\hline GP3114 & $\operatorname{trpC2} \Delta c d a S::$ ermC $\triangle c d a A:: c a t$ amyE::(P $P_{x y \mid}-a c c D A$ spec $)$ & NJ61 $\rightarrow$ GP2032 \\
\hline GP3115 & $\begin{array}{l}\operatorname{trpC2} \Delta c d a S:: e r m C \Delta c d a A:: c a t \text { pls } C^{\prime} \Omega\left(P_{x y \mid}-p l s C \text { spc }\right) \\
\text { amyE::(Pxyl-xylR tet }) \Delta d i s A:: k a n\end{array}$ & $\mathrm{GP} 2782 \rightarrow \mathrm{GP} 3112$ \\
\hline GP3116 & $\operatorname{trpC2} \Delta c$ daS::spc disA::tet acc $A_{A 229 V}$ phleo yfk $C_{R 42 W}$ kan & GP2798 $\rightarrow$ GP3110 \\
\hline GP3117 & $\begin{array}{l}\operatorname{trpC2} \triangle c d a S:: s p c \text { disA::tet acc } A_{A 229 v} \text { phleo yfk } C_{R 42 w} \text { kan } \\
\triangle c d a A:: c a t\end{array}$ & GP997 $\rightarrow$ GP3116 \\
\hline GP3118 & $\begin{array}{l}\left.\operatorname{trpC2} \triangle c d a S:: e r m C \triangle c d a A:: c a t \text { amyE::(P } P_{x y l}-a c c D A \text { spec }\right) \\
\Delta d i s A:: k a n\end{array}$ & $\mathrm{GP} 2782 \rightarrow \mathrm{GP} 3114$ \\
\hline GP3119 & $\operatorname{trpC2} \Delta c$ daS::ermC $\triangle$ disA::tet lacA::( $P_{\text {deqQ }}$ darB kan) & GP3104 $\rightarrow$ GP991 \\
\hline
\end{tabular}




\begin{tabular}{|c|c|c|}
\hline Strain & Genotype & Reference/Construction \\
\hline GP3120 & $\begin{array}{l}\operatorname{trpC2} \triangle c \text { daS::ermC } \triangle \text { disA::tet lacA::( } P_{\text {deqa }} \text { darB kan) } \\
\Delta c d a A:: c a t\end{array}$ & GP997 $\rightarrow$ GP3119 \\
\hline GP3121 & $\begin{array}{l}\operatorname{trpC2} \Delta c d a A:: \text { cat } \triangle c \text { daS::ermC } \triangle \text { disA::tet yua } C_{f s} c c p N_{f s} \\
f l i H_{f s} o p p D_{f s}\end{array}$ & Suppressor of GP2222 \\
\hline GP3122 & $\operatorname{trpC2} \Delta a h r C::$ phleo & $\mathrm{LFH} \rightarrow 168$ \\
\hline GP3123 & $\operatorname{trpC2} \Delta k \operatorname{trAB}::$ kan $\triangle$ kimA::cat $\triangle$ darA::spc $\Delta g / t A B::$ tet & GP807 $\rightarrow$ GP2461 \\
\hline GP3124 & $\operatorname{trp} C 2 \Delta k \operatorname{tr} A B:: k a n \Delta k i m A::$ cat $\Delta g l t A B::$ tet & GP807 $\rightarrow$ GP2165 \\
\hline GP3125 & $\begin{array}{l}\operatorname{trpC2} \Delta k \operatorname{kt} A B:: k a n \triangle k i m A:: \text { cat } \triangle \text { darA::spc } \Delta g l t A B:: \text { tet } \\
\Delta a h r C:: \text { phleo }\end{array}$ & GP3122 $\rightarrow$ GP3123 \\
\hline GP3126 & $\operatorname{trp} C 2 \Delta k \operatorname{tr} A B:: k a n \Delta k i m A:: c a t \Delta g l t A B::$ tet $\triangle a h r C::$ phleo & GP3122 $\rightarrow$ GP3124 \\
\hline GP3127 & $\operatorname{trpC2} \Delta k \operatorname{trC}::$ tet $\Delta k i m A::$ cat $\Delta k \operatorname{tr} B:: k a n$ & Krüger et al., 2020b \\
\hline GP3128 & $\operatorname{trpC2} \Delta g / t A B:: t e t$ ganA::(gdhA $\left.A_{E c o}\right):: k a n$ & pGP3000 $\rightarrow$ GP807 \\
\hline GP3129 & $\begin{array}{l}\operatorname{trpC2} \Delta c d a A:: c a t \Delta c d a S:: e r m C \Delta \text { disA::tet yuaC } f s \\
c c p N_{D 42 / L 43 \rightarrow E 42}\end{array}$ & Suppressor of GP2222 \\
\hline GP3130 & $\operatorname{trpC2} \triangle c d a S::$ ermC $\triangle$ disA::tet $\triangle c i t M:: k a n$ & GP2760 $\rightarrow$ GP991 \\
\hline GP3132 & $\begin{array}{l}\operatorname{trpC2} \Delta c \text { daS::ermC } \triangle \text { disA::tet } \triangle \text { darB-ccpC::phleo } \\
\text { lacA::(P } P_{\text {deqa }} \text { darB kan) }\end{array}$ & GP3057 $\rightarrow$ GP991 \\
\hline GP3133 & $\begin{array}{l}\operatorname{trp} C 2 \Delta c \text { daS::ermC } \triangle \text { disA::tet } \triangle \text { darB-ccpC::phleo } \\
\text { lacA::(P } P_{\text {deqQ }} \text { darB kan) } \Delta c d a A:: c a t\end{array}$ & GP997 $\rightarrow$ GP3132 \\
\hline GP3134 & $\operatorname{trpC2} \Delta c d a S::$ ermC $\Delta$ disA::tet $\Delta c c p C:: k a n$ & GP1445 $\rightarrow$ GP991 \\
\hline GP3135 & $\operatorname{trp} C 2 \Delta c d a S:: e r m C \Delta$ disA::tet $\triangle c c p C:: k a n \Delta c d a A:: c a t$ & GP997 $\rightarrow$ GP3134 \\
\hline GP3136 & $\begin{array}{l}\operatorname{trpC} 2 \Delta c d a S:: s p c \triangle d i s A:: \text { tet } \triangle \text { darB::ermC lacA::(P dequ darB } \\
\text { kan) }\end{array}$ & GP3104 $\rightarrow$ GP2777 \\
\hline GP3137 & $\begin{array}{l}\operatorname{trpC} \triangle \Delta c \text { daS::spc } \triangle \text { disA::tet } \triangle \text { darB::ermC lacA::(P deqa darB } \\
\text { kan) } \triangle c d a A:: c a t\end{array}$ & GP997 $\rightarrow$ GP3136 \\
\hline GP3138 & $\operatorname{trp} C 2 \Delta c$ daS::ermC $\triangle$ disA::tet $\Delta c c p N:: c a t$ & GP706 $\rightarrow$ GP991 \\
\hline GP3139 & $\operatorname{trp} C 2 \Delta c$ daS::ermC $\Delta$ disA::tet $\Delta c c p N:: c a t \Delta c d a A:: s p e c$ & GP94 $\rightarrow$ GP3138 \\
\hline GP3140 & $\begin{array}{l}\operatorname{trpC2} \triangle c \text { daS::ermC } \triangle \text { disA::tet lacA::(P } P_{\text {deqQ }} \text { darB kan) } \\
\text { amyE::(ccpC-`lacZ cat) }\end{array}$ & GP210 $\rightarrow$ GP3119 \\
\hline GP3142 & $\begin{array}{l}\operatorname{trpC2} \Delta c d a S:: s p c \triangle d i s A:: \text { tet } \triangle \text { darB::ermC amyE::(ccpC- } \\
\text { YacZ cat) }\end{array}$ & GP210 $\rightarrow$ GP2777 \\
\hline GP3143 & $\begin{array}{l}\operatorname{trpC2} \triangle c d a S:: s p c \Delta d i s A:: t e t \triangle d a r B:: e r m C \text { amyE::(ccpC- } \\
\text { YacZ cat) } \triangle c d a A:: k a n\end{array}$ & $\mathrm{GP} 2790 \rightarrow \mathrm{GP} 3142$ \\
\hline GP3144 & $\operatorname{trpC2} \triangle \operatorname{ccpN}:: c a t$ lacA::(P deqQ darB kan) & GP3104 $\rightarrow$ GP1128 \\
\hline GP3145 & $\operatorname{trpC2} \Delta c d a S:: e r m C \Delta d i s A::$ tet amyE::(ccpC-'lacZ cat) & GP210 $\rightarrow$ GP991 \\
\hline GP3146 & $\begin{array}{l}\operatorname{trpC2} \triangle c \text { daS::ermC } \triangle \text { disA::tet amyE::(ccpC-'lacZ cat) } \\
\triangle c d a A:: \text { spec }\end{array}$ & GP94 $\rightarrow$ GP3145 \\
\hline GP3147 & $\operatorname{trpC2}$ amyE::(citZ- `acZ cat) & pGP3310 $\rightarrow 168$ \\
\hline GP3148 & $\operatorname{trpC2} \triangle c c p C:: k a n$ amyE::(citZ-`lacZ cat) & pGP3310 $\rightarrow$ GP1442 \\
\hline GP3149 & $\operatorname{trpC2} \Delta y k u L-c c p C:: p h l e o$ lacA::( $P_{\text {deqQ }}$ darB kan) & GP3104 $\rightarrow$ GP2800 \\
\hline GP3150 & $\operatorname{trpC2} \triangle f s r A:: s p e c$ & $\mathrm{HB7385} \rightarrow 168$ \\
\hline
\end{tabular}




\begin{tabular}{|c|c|c|}
\hline Strain & Genotype & Reference/Construction \\
\hline GP3401 & trpC2 amyE::(citB-`lacZ cat) $\triangle f s r A:: s p e c$ & HB7385 $\rightarrow$ GP205 \\
\hline GP3402 & $\operatorname{trpC2}$ amyE::(citB- 'lacZ cat) $\triangle c c p C::$ kan $\triangle f s r A::$ spec & HB7385 $\rightarrow$ GP206 \\
\hline GP3403 & $\operatorname{trpC2}$ amyE::(citZ-`acZ cat) $\Delta f s r A:: s p e c$ & HB7385 $\rightarrow$ GP3147 \\
\hline GP3404 & $\operatorname{trpC2}$ amyE::(citZ- 'lacZ cat) $\Delta c c p C:: k a n \triangle f s r A:: s p e c$ & $\mathrm{HB} 7385 \rightarrow \mathrm{GP} 3148$ \\
\hline GP3405 & $\operatorname{trpC2}$ amyE::(citB- 'lacZ cat) $\triangle$ citM::spec & GP2761 $\rightarrow$ GP205 \\
\hline GP3406 & $\operatorname{trpC2}$ amyE::(citB- 'lacZ cat) $\Delta c i t Z:: s p e c$ & GP797 $\rightarrow$ GP205 \\
\hline GP3407 & $\operatorname{trp} C 2 \Delta c c p N:: k a n$ & $\mathrm{LFH} \rightarrow 168$ \\
\hline GP3408 & $\operatorname{trpC2}$ pGP3306 (darB $\left.{ }^{+}\right)$ & $\begin{array}{l}\text { pGP3306 } \rightarrow 168, \\
\text { suppressor mutant }\end{array}$ \\
\hline GP3409 & $\operatorname{trpC2} \Delta d a r B:: c a t$ & $\mathrm{LFH} \rightarrow 168$ \\
\hline GP3410 & $\operatorname{trpC2}$ amyE::(pta- 'lacZ cat) & BSIP1114 $\rightarrow 168$ \\
\hline GP3411 & $\operatorname{trpC2} / d h-{ }^{`} / a c Z$ cat & LUW276 $\rightarrow 168$ \\
\hline GP3412 & $\operatorname{trpC2}$ amyE::(pta- ҮacZ cat) $\triangle$ darB::ermC & GP2769 $\rightarrow$ GP3410 \\
\hline GP3413 & $\operatorname{trpC2}$ Idh- 'lacZ cat $\triangle$ darB::ermC & GP2769 $\rightarrow$ GP3410 \\
\hline GP3414 & $\operatorname{trpC2} \Delta c d a A:: c a t \Delta c d a S:: e r m C \Delta$ disA::tet $k \operatorname{tr} C_{\text {frameshift }}$ & Krüger et al., 2020b \\
\hline GP3415 & $\operatorname{trpC2} \operatorname{trpC2}$ amyE::(odhA- YacZ cat) & pGP3106 $\rightarrow 168$ \\
\hline GP3416 & $\operatorname{trpC2}$ amyE::(odhA-'lacZ cat) $\triangle$ darB::ermC & GP2769 $\rightarrow 168$ \\
\hline GP3417 & NClB3610 $\Delta$ darB::cat & $\mathrm{GP} 3409 \rightarrow \mathrm{NClB} 3610$ \\
\hline GP3418 & NCIB3610 $\Delta \mathrm{gdpP}:: s p c \Delta p g p H:: e r m C \Delta d a r B:: c a t$ & GP3409 $\rightarrow$ GP2164 \\
\hline GP3419 & $\operatorname{trpC2} \Delta r e l:: c a t$ & Krüger et al., 2020c \\
\hline GP3420 & NCIB3610 $\Delta \mathrm{gdpP}:: s p c \Delta p g p H:: e r m C \Delta$ sunA::kan & GP1563 $\rightarrow$ GP2164 \\
\hline GP3421 & $\operatorname{trpC2} \Delta c c p C:: k a n \Delta r e l:: c a t$ & GP3419 $\rightarrow$ GP1442 \\
\hline GP3422 & $\operatorname{trpC2}$ amyE::(ilvA- 'lacZ cat) $\triangle$ darB::ermC & GP2769 $\rightarrow$ GP327 \\
\hline GP3423 & $\operatorname{trpC2}$ amyE::(ilvD- YacZ cat) $\triangle$ darB::ermC & GP2769 $\rightarrow$ GP330 \\
\hline GP3424 & $\operatorname{trpC2} \Delta$ sasA::spec & $\mathrm{LFH} \rightarrow 168$ \\
\hline GP3425 & $\operatorname{trpC2} \Delta s a s B:: p h l e o$ & $\mathrm{LFH} \rightarrow 168$ \\
\hline GP3426 & $\operatorname{trpC2}$ lacA::(Pxyl rel kan) & pGP3329 $\rightarrow 168$ \\
\hline GP3427 & $\operatorname{trpC2} \Delta$ rel::cat lacA::(Pxyl rel kan) & pGP3329 $\rightarrow$ GP3419 \\
\hline GP3428 & $\operatorname{trp} C 2 \Delta c c p C:: k a n$ arel::cat $\triangle$ darB:::ermC & GP2769 $\rightarrow$ GP3421 \\
\hline GP3429 & $\operatorname{trpC2}$ rel-6xHis-cat & Krüger et al., 2020c \\
\hline GP3431 & $\operatorname{trpC2} \Delta r e l:: p h l e o$ & $\mathrm{LFH} \rightarrow 168$ \\
\hline GP3432 & $\operatorname{trp} C 2 \Delta$ sunT::spec & $\mathrm{LFH} \rightarrow 168$ \\
\hline GP3433 & $\operatorname{trpC2}$ amyE::(citB-YacZ cat) $\Delta \operatorname{cod} Y:: s p e c$ & GP2473 $\rightarrow$ GP205 \\
\hline GP3435 & $\operatorname{trpC2}$ amyE::(ilvA-YacZ cat) $\Delta c o d Y:: s p e c$ & GP2473 $\rightarrow$ GP327 \\
\hline GP3436 & $\operatorname{trpC2} \Delta c d a S::$ ermC $\Delta$ disA::tet $\Delta$ rel:::phleo & GP3431 $\rightarrow$ GP991 \\
\hline GP3437 & $\operatorname{trpC2} \Delta c$ daS::spc $\triangle$ disA::tet $\triangle$ darB::ermC $\Delta$ rel::phleo & GP3431 $\rightarrow$ GP2769 \\
\hline GP3438 & $\operatorname{trpC2} \Delta c$ daS::ermC $\Delta$ disA::tet $\Delta r e l:: p h l e o \Delta c d a A:: c a t$ & GP997 $\rightarrow$ GP3436 \\
\hline GP3439 & $\begin{array}{l}\operatorname{trpC2} \Delta c \text { daS::spc } \triangle \text { disA::tet } \triangle \text { darB::ermC } \Delta \text { rel::phleo } \\
\Delta c d a A:: \text { cat }\end{array}$ & GP997 $\rightarrow$ GP3437 \\
\hline GP3440 & $\operatorname{trpC2} \Delta c$ daS::ermC $\triangle$ disA::tet aimA-3x FLAG spec & GP3062 $\rightarrow$ GP991 \\
\hline
\end{tabular}




\begin{tabular}{|c|c|c|}
\hline Strain & Genotype & Reference/Construction \\
\hline GP3443 & $\operatorname{trpC2} \Delta$ rel::cat lacA::(P $P_{x y \mid}$ rel-D264G kan) & pGP3335 $\rightarrow$ GP3419 \\
\hline GP3445 & $\operatorname{trpC2} \Delta k \operatorname{tr} A B:: k a n \Delta k i m A:: c a t \Delta g l t T:: e r m C$ & GP2247 $\rightarrow$ GP2165 \\
\hline GP3446 & $\operatorname{trpC2} \Delta k \operatorname{trAB}:: k a n \Delta k i m A:: c a t \Delta a i m A:: p h l e o$ & GP3054 $\rightarrow$ GP2165 \\
\hline GP3447 & $\operatorname{trpC2} \Delta$ ktrAB::kan $\triangle$ kimA::cat $\triangle a i m A::$ phleo $\Delta$ gltT::ermC & GP2247 $\rightarrow$ GP3446 \\
\hline GP3448 & $\operatorname{trpC2}$ amyE::(citB-`lacZ cat) $\Delta c c p C:: k a n \Delta c o d Y:: s p e c$ & GP2473 $\rightarrow$ GP206 \\
\hline GP3449 & $\operatorname{trpC2} \Delta c$ daS::ermC $\Delta$ disA::tet $\Delta$ codY::spec & GP2473 $\rightarrow$ GP991 \\
\hline GP3451 & $\operatorname{trpC2} \Delta$ rel::cat lacA::(P $P_{x y \mid}$ rel-SYN-RRM kan) & pGP3331 $\rightarrow$ GP3419 \\
\hline GP3452 & $\operatorname{trpC2} \Delta$ rel:::cat lacA::(P $P_{x y l}$ rel-HYD-SYN kan) & pGP3334 $\rightarrow$ GP3419 \\
\hline GP3453 & $\operatorname{trpC2} \Delta \operatorname{cod} Y:: p h l e o$ & $\mathrm{LFH} \rightarrow 168$ \\
\hline GP3454 & $\operatorname{trpC2} \Delta \operatorname{cod} Y:: c a t$ & LFH $\rightarrow 168$ \\
\hline GP3455 & $\operatorname{trpC2}$ codY-G146V::phleo & LFH $\rightarrow 168$ \\
\hline GP3456 & $\operatorname{trpC2} \operatorname{cod} Y-G 146 \mathrm{~V}:: \mathrm{cat}$ & LFH $\rightarrow 168$ \\
\hline GP3460 & $\operatorname{trpC2} \Delta y d z A-m n t H:: e r m C \Delta r e l:: p h l e o$ & GP3431 $\rightarrow$ GP2756 \\
\hline GP3461 & $\operatorname{trpC2} \Delta k \operatorname{trC}::$ tet $\Delta r e l:: c a t$ & GP3419 $\rightarrow$ GP2079 \\
\hline GP3462 & $\operatorname{trpC2} \Delta$ ktrC::tet $\Delta$ rel:::cat lacA::(P $P_{x y l}$ rel kan) & GP3427 $\rightarrow$ GP3461 \\
\hline GP3464 & $\begin{array}{l}\operatorname{trpC2} \Delta c d a A:: c a t \Delta c d a S:: e r m C \Delta \text { disA::tet pls } C_{P 110 s} \\
\text { flhA }_{\text {frameshift }}\end{array}$ & Krüger et al., 2020a \\
\hline GP3465 & $\operatorname{trpC2}$ ktrD-3x FLAG spec & pGP2792 $\rightarrow 168$ \\
\hline GP3466 & $\operatorname{trpC2}$ rel-6xHis-cat ktrD-3x FLAG spec & pGP2792 $\rightarrow$ GP3429 \\
\hline GP3467 & $\operatorname{trpC2}$ sasB-6xHis-cat & LFH $\rightarrow 168$ \\
\hline GP3468 & $\operatorname{trpC2} \Delta$ sasA::spec $\Delta$ rel:::cat $\triangle$ sasB::phleo & GP3425 $\rightarrow$ GP3471 \\
\hline GP3469 & $\operatorname{trpC2}$ ktrD-3x FLAG spec sasB-6xHis-cat & GP3467 $\rightarrow$ GP3465 \\
\hline GP3470 & $\operatorname{trpC2} \triangle$ sasA::spec $\triangle$ sasB::phleo & GP3425 $\rightarrow$ GP3424 \\
\hline GP3471 & $\operatorname{trpC2} \Delta$ sasA::spec $\Delta$ rel:::cat & GP3419 $\rightarrow$ GP3424 \\
\hline GP3472 & $\operatorname{trpC2} \Delta$ rel::cat $\Delta$ sasB::phleo & GP3425 $\rightarrow$ GP3419 \\
\hline GP3473 & 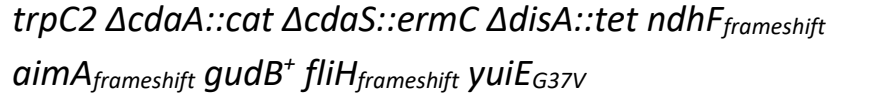 & Krüger et al., 2020a \\
\hline GP3474 & $\begin{array}{l}\operatorname{trpC2} \Delta c d a A:: c a t \Delta c d a S:: e r m C \Delta d i s A:: \text { tet } n d h F(\mathrm{fs}) \\
\operatorname{aim}_{\text {frameshift }} y k p A_{\text {frameshift }} k \operatorname{kt} C_{\text {frameshift }} \text { fli }_{\text {frameshift }} \text { fliH }_{\text {silent }}\end{array}$ & Krüger et al., 2020a \\
\hline GP3475 & $\operatorname{trpC2} \Delta$ cdaS::ermC $\Delta$ disA::tet $\Delta$ rel:::phleo $\Delta$ sasA::spec & GP3424 $\rightarrow$ GP3436 \\
\hline GP3476 & $\operatorname{trpC2} \Delta$ cdaS::ermC $\triangle$ disA::tet $\triangle$ sasB::phleo & GP3425 $\rightarrow$ GP991 \\
\hline GP3477 & $\operatorname{trpC2} \Delta$ cdaS::ermC $\Delta$ disA::tet $\triangle$ sasB::phleo $\Delta$ rel::cat & GP3419 $\rightarrow$ GP3476 \\
\hline GP3478 & $\operatorname{trpC2} \Delta$ cdaS::ermC $\triangle$ disA::tet $\triangle$ sasB::phleo $\triangle$ sasA::spec & GP3424 $\rightarrow$ GP3476 \\
\hline GP3479 & $\operatorname{trpC2} \Delta$ cdaS::ermC $\Delta$ disA::tet $\triangle$ sasA::spec & GP3424 $\rightarrow$ GP991 \\
\hline GP3480 & $\operatorname{trpC2} \triangle c$ daS::ermC $\triangle$ disA::tet $\Delta r e l:: c a t$ & GP3419 $\rightarrow$ GP991 \\
\hline GP3481 & $\operatorname{trpC2} \Delta c d a S:: e r m C \Delta$ disA::tet $\Delta$ sasA::spec $\Delta c d a A:: k a n$ & GP2790 $\rightarrow$ GP3479 \\
\hline GP3482 & $\begin{array}{l}\text { trpC2 } \triangle c \text { daS::ermC } \triangle \text { disA::tet } \triangle \text { sasB::phleo } \triangle \text { sasA::spec } \\
\Delta c d a A:: k a n\end{array}$ & GP2790 $\rightarrow$ GP3478 \\
\hline GP3483 & $\operatorname{trpC2} \Delta c$ daS::ermC $\triangle$ disA::tet $\Delta$ sasB::phleo $\triangle c d a A:: k a n$ & GP2790 $\rightarrow$ GP3476 \\
\hline
\end{tabular}




\begin{tabular}{|c|c|c|}
\hline Strain & Genotype & Reference/Construction \\
\hline GP3484 & $\begin{array}{l}\operatorname{trpC2} \triangle c d a S:: e r m C \Delta \text { disA::tet } \Delta \text { rel::phleo } \triangle \text { sasA::spec } \\
\triangle c d a A:: k a n\end{array}$ & GP2790 $\rightarrow$ GP3475 \\
\hline GP3485 & $\begin{array}{l}\operatorname{trp} C 2 \Delta c d a S:: e r m C \Delta \text { disA::tet } \triangle \text { sasB::phleo } \Delta \text { rel::cat } \\
\Delta c d a A:: k a n\end{array}$ & GP2790 $\rightarrow$ GP3477 \\
\hline GP3486 & $\begin{array}{l}\operatorname{trpC2} \Delta c d a A:: c a t \Delta c d a S:: e r m C \Delta d i s A: \text { tet nhaK } \\
\text { aimA }_{\text {frameshift }}\end{array}$ & Krüger et al., 2020a \\
\hline GP3487 & $\begin{array}{l}\operatorname{trpC2} \Delta c d a A:: c a t \Delta c d a S:: e r m C \Delta d i s A:: \text { tet } n h a K_{\mathrm{S} 187 \mathrm{~F}} \\
\text { aimA }_{\text {frameshift }}\end{array}$ & Krüger et al., 2020a \\
\hline GP3488 & $\begin{array}{l}\operatorname{trpC2} \Delta c d a A:: c a t \Delta c d a S:: e r m C \Delta d i s A: \text { tet nhaK } K_{\mathrm{s} 187 \mathrm{~F}} \\
\operatorname{aimA}_{\text {frameshift }}\end{array}$ & Krüger et al., 2020a \\
\hline GP3489 & $\begin{array}{l}\operatorname{trpC2} \triangle c d a A:: \text { cat } \triangle c d a S:: e r m C \Delta \text { disA::tet nhaK }{ }_{\mathrm{S} 187 \mathrm{~F}} \\
\operatorname{aimA}_{P 279 H}\end{array}$ & Krüger et al., 2020a \\
\hline GP3490 & $\begin{array}{l}\operatorname{trpC2} \Delta c d a A:: c a t \Delta c d a S:: e r m C \Delta d i s A:: \text { tet } n h a K_{\mathrm{S} 187 \mathrm{~F}} \\
\operatorname{aimA}_{1164 \mathrm{~N}}\end{array}$ & Krüger et al., 2020a \\
\hline GP3491 & $\begin{array}{l}\operatorname{trpC2} \Delta c d a A:: c a t \Delta c d a S:: e r m C \Delta d i s A: \text { tet nhaK } K_{\mathrm{S} 187 \mathrm{~F}} \\
\text { aimA }_{\text {frameshift }}\end{array}$ & Krüger et al., 2020a \\
\hline GP3492 & $\begin{array}{l}\operatorname{trpC2} \triangle c d a A:: \text { cat } \Delta c d a S:: e r m C \Delta \text { disA::tet nhaK } K_{\mathrm{S} 187 \mathrm{~F}} \\
\text { aimA }_{\text {frameshift }}\end{array}$ & Krüger et al., 2020a \\
\hline GP3493 & $\begin{array}{l}\operatorname{trpC2} \Delta c d a A:: \text { cat } \triangle c d a S:: e r m C \Delta \text { disA::tet } n h a K_{\text {S187F }} \\
\text { aimA }_{\text {frameshift }}\end{array}$ & Krüger et al., 2020a \\
\hline GP3494 & $\begin{array}{l}\operatorname{trpC2} \Delta c d a A:: c a t \Delta c d a S:: e r m C \Delta d i s A: \text { tet } n h a K_{\mathrm{S} 187 \mathrm{~F}} \\
\operatorname{aimA}_{\text {s106P }}\end{array}$ & Krüger et al., 2020a \\
\hline GP3495 & $\begin{array}{l}\operatorname{trpC2} \Delta c d a A:: c a t \Delta c d a S:: e r m C \Delta \text { disA::tet nhaK } K_{\mathrm{S} 187 \mathrm{~F}} \\
\operatorname{aimA}_{N 349 K}\end{array}$ & Krüger et al., 2020a \\
\hline GP3496 & $\begin{array}{l}\operatorname{trpC2} \Delta c d a A:: c a t \Delta c d a S:: e r m C \Delta d i s A: \text { tet } n h a K_{\mathrm{S} 187 \mathrm{~F}} \\
\operatorname{aimA}_{\text {frameshift }}\end{array}$ & Krüger et al., 2020a \\
\hline GP3497 & $\begin{array}{l}\operatorname{trpC2} \triangle c d a A:: \text { cat } \triangle c d a S:: e r m C \Delta \text { disA::tet } n h a K_{\text {S187F }} \\
\text { aimA }_{\text {frameshift }}\end{array}$ & Krüger et al., 2020a \\
\hline GP3498 & $\begin{array}{l}\operatorname{trpC2} \Delta c d a A:: c a t \triangle c d a S:: e r m C \Delta d i s A: \text { tet } n h a K_{\mathrm{S} 187 \mathrm{~F}} \\
\operatorname{aimA}_{G 70 R}\end{array}$ & Krüger et al., 2020a \\
\hline GP3499 & $\begin{array}{l}\operatorname{trpC2} \triangle c d a A:: \text { cat } \Delta c d a S:: e r m C \Delta \text { disA::tet nhaK } K_{\mathrm{S} 187 \mathrm{~F}} \\
\operatorname{aimA}_{\text {Insertion after a } 226(+V A)}\end{array}$ & Krüger et al., 2020a \\
\hline GP3500 & $\begin{array}{l}\operatorname{trpC2} \Delta c d a A:: c a t \Delta c d a S:: e r m C \Delta d i s A:: \text { tet } n h a K_{\mathrm{S} 187 \mathrm{~F}} \\
\operatorname{aimA}_{\text {frameshift }}\end{array}$ & Krüger et al., 2020a \\
\hline GP3701 & $\begin{array}{l}\operatorname{trpC2} \Delta c d a A:: \text { cat } \triangle c d a S:: e r m C \Delta \text { disA::tet nhaK } K_{\mathrm{S} 187 \mathrm{~F}} \\
\text { aimA }_{\text {frameshift }}\end{array}$ & Krüger et al., 2020a \\
\hline GP3702 & $\begin{array}{l}\operatorname{trpC2} \Delta c \text { daS::spc } \Delta \text { disA::tet } \triangle \text { darB::ermC } \triangle c d a A:: c a t \\
k_{t r} C_{\text {frameshift }} \text { kimA } A_{\triangle 354-364} \text { darA }_{\text {frameshift }}\end{array}$ & Krüger et al., 2020a \\
\hline GP3703 & $\begin{array}{l}\operatorname{trpC2} \Delta c \text { daS::spc } \Delta \text { disA::tet } \Delta \text { darB::ermC } \Delta c d a A:: \text { cat } \\
\text { ktrC }_{\text {frameshift }} \text { kimA }{ }_{\Delta 354-364} \text { darA }_{\text {frameshift }}\end{array}$ & Krüger et al., 2020a \\
\hline GP3704 & $\begin{array}{l}\text { trpC2 } \Delta \text { cdaS::ermC } \triangle \text { disA::tet } \triangle \text { sasB::phleo } \triangle \text { sasA::spec } \\
\Delta r e l:: c a t\end{array}$ & GP3419 $\rightarrow$ GP3478 \\
\hline
\end{tabular}




\begin{tabular}{lll}
\hline Strain & Genotype & Reference/Construction \\
\hline GP3705 & trpC2 $\Delta c$ cdaS::ermC $\Delta$ disA::tet $\Delta$ sasB::phleo $\Delta$ sasA::spec & GP2790 $\rightarrow$ GP3704 \\
& drel::cat $\Delta c$ cdaA::kan & \\
\hline
\end{tabular}

\subsection{Plasmids used in this study}

\begin{tabular}{|c|c|c|}
\hline Name & Purpose & Reference \\
\hline $\mathrm{p} 25-\mathrm{N}$ & $\begin{array}{l}\text { Fusion of the target protein to the } \mathrm{N} \text {-terminus of the } \mathrm{T} 25 \\
\text { domain }\end{array}$ & Karimova et al., 1998 \\
\hline pGP380 & $\begin{array}{l}\text { Constitutive overexpression of } \mathrm{N} \text {-terminally Strep-tagged } \\
\text { proteins in } B \text {. subtilis }\end{array}$ & Herzberg et al., 2007 \\
\hline pGP382 & $\begin{array}{l}\text { Constitutive overexpression of C-terminally Strep-tagged } \\
\text { proteins in B. subtilis }\end{array}$ & Herzberg et al., 2007 \\
\hline pGP706 & IPTG inducible expression of 6x-His-tagged CcpC in E. coli & Blencke et al., 2006 \\
\hline pGP2594 & IPTG inducible expression of 6x-His-tagged KtrA in E. coli & Gundlach et al., 2019 \\
\hline pGP2906 & IPTG inducible expression of 6x-His-tagged KhtT in E. coli & Gundlach et al., 2019 \\
\hline pGP2907 & IPTG inducible expression of $6 \mathrm{x}$-His-tagged $\mathrm{KtrC}$ in E. coli & Gundlac \\
\hline pGP2908 & IPTG inducible expression of 6x-His-tagged YvrC in E. coli & Gundlach et al., 2019 \\
\hline pGP2909 & IPTG inducible expression of 6x-His-tagged YjbQ in E. coli & Gundlach et al., 2019 \\
\hline pGP2913 & IPTG inducible expression of $6 x$-His-tagged KimA in E. coli & Gundlach et al., 2019 \\
\hline pGP2922 & IPTG inducible expression of 6x-His-tagged AcuB in E. coli & Gundlach et al., 2019 \\
\hline pGP2923 & IPTG inducible expression of 6x-His-tagged $\mathrm{CcpN}$ in E. coli & Gundlach et al., 2019 \\
\hline pGP2924 & IPTG inducible expression of 6x-His-tagged OpuAA in E. coli & Gundlach et a \\
\hline pGP2927 & IPTG inducible expression of $6 x$-His-tagged MgtE in E. coli & Gundlach et al., 2019 \\
\hline pGP2928 & IPTG inducible expression of 6x-His-tagged YhdT in E. coli & Gundlach et al., 2019 \\
\hline pGP2929 & IPTG inducible expression of 6x-His-tagged DarB in E. coli & Gundlach et al., 2019 \\
\hline pGP2930 & IPTG inducible expression of 6x-His-tagged YhcV in E. coli & Gundlach et al., 2019 \\
\hline pGP2931 & IPTG inducible expression of 6x-His-tagged YhdP in E. coli & Gundlach et al., 2019 \\
\hline pGP2932 & YrkA in E. coli & 2019 \\
\hline pGP2933 & oression of $6 x-H i s-t$ & Gundlach et al., 2019 \\
\hline pGP2934 & IPTG inducible expression of 6x-His-tagged YlbB in E. coli & Gundlach et al., 2019 \\
\hline pGP2935 & IPTG inducible expression of 6x-His-tagged YqhB in E. coli & Gundlach et al., 2019 \\
\hline pGP2936 & IPTG inducible expression of 6x-His-tagged YugS in E. coli & Gundlach et al., 2019 \\
\hline pGP2937 & IPTG inducible expression of 6x-His-tagged GuaB in E. coli & Gundlach et al., 2019 \\
\hline
\end{tabular}




\begin{tabular}{|c|c|c|}
\hline Name & Purpose & Reference \\
\hline pGP2938 & IPTG inducible expression of $6 x$-His-tagged OpuBA in E. coli & Gundlach et al., 2019 \\
\hline pGP2939 & IPTG inducible expression of $6 x$-His-tagged OpuCA in E. coli & Gundlach et al., 2019 \\
\hline pKT25 & $\begin{array}{l}\text { Fusion of the target protein to the C-terminus of the T25 } \\
\text { domain }\end{array}$ & Karimova et al., 1998 \\
\hline pKT25zip & $\begin{array}{l}\text { Control plasmid, expressing leucine zipper fused to T25 } \\
\text { fragment of adenylate cyclase }\end{array}$ & Karimova et al., 1998 \\
\hline pUT18 & $\begin{array}{l}\text { Fusion of the target protein to the } \mathrm{N} \text {-terminus of the T18 } \\
\text { domain }\end{array}$ & Karimova et al., 1998 \\
\hline pUT18C & $\begin{array}{l}\text { Fusion of the target protein to the C-terminus of the T18 } \\
\text { domain }\end{array}$ & Karimova et al., 1998 \\
\hline pUT18Czip & $\begin{array}{l}\text { Control plasmid, expressing leucine zipper fused to T18 } \\
\text { fragment of adenylate cyclase }\end{array}$ & Karimova et al., 1998 \\
\hline pVHP186 & $\begin{array}{l}\text { IPTG-inducible overexpression of } \mathrm{N} \text {-terminally } 10 \mathrm{x} \text {-His- } \\
\text { tagged Rel in E. coli }\end{array}$ & Takada et al., 2020 \\
\hline pWH844 & $\begin{array}{l}\text { IPTG-inducible overexpression of N-terminally His-tagged } \\
\text { proteins in E. coli }\end{array}$ & Schirmer et al., 1997 \\
\hline
\end{tabular}

\subsection{Plasmids constructed in this study}

\begin{tabular}{|c|c|c|c|}
\hline Name & Vector & Insert & Reference \\
\hline pGP635 & pGP380/Xbal+Pstl & $\begin{array}{l}\text { PCR-product darB, TK01/TK02 } \\
\text { (Xbal+Pstl) }\end{array}$ & Krüger et al., 2020c \\
\hline pGP767 & pGP382/Xbal+Pstl & $\begin{array}{l}\text { PCR-product darB, TK03/TK04 } \\
\text { (Xbal+Pstl) }\end{array}$ & Krüger et al., 2020c \\
\hline pGP2965 & pAC6/EcoRI+BamHI & $\begin{array}{l}\text { citM- lacZ-Fusion; LK55/LK56 } \\
\text { (EcoRI+BamHI) }\end{array}$ & This study \\
\hline pGP2966 & pAC6/EcoRI+BamHI & $\begin{array}{l}\text { citM-`lacZ-fusion amplified from } \\
\text { GP2811 [citM ( } \Delta \text { cre-site)]; } \\
\text { LK55/LK56 (EcoRI+BamHI) }\end{array}$ & This study \\
\hline pGP2967 & pAC6/EcoRI+BamHI & $\begin{array}{l}\text { citM- lacZ-fusion amplified from } \\
\text { GP2812 [citM (cre-site TGTA- } \\
\text { >TATA)]; LK55/LK56 (EcoRI+BamHI) }\end{array}$ & This study \\
\hline pGP2968 & pGP1331/EcoRI+Pstl & $\begin{array}{l}\text { PCR-product 3`-Ende von citM } \\
\text { LK57/LK58 (EcoRI+Pstl) }\end{array}$ & This study \\
\hline pGP2969 & pGP1331/EcoRI+Pstl & $\begin{array}{l}\text { PCR-product 3`-Ende von mgtE } \\
\text { LK59/LK60 (EcoRI+Pstl) }\end{array}$ & This study \\
\hline pGP2970 & pGP172/Sacl+Kpnl & $\begin{array}{l}\text { PCR-product cytosolic domain (0- } \\
277 \text { aa) mgtE LK63/LK64 (Sacl+Kpnl) }\end{array}$ & This study \\
\hline pGP2971 & pGP172/Sacl+Kpnl & $\begin{array}{l}\text { PCR-product cytosolic domain (0- } \\
277 \text { aa) } m g t E^{\text {F229A }, \text { LK63/LK64 }} \\
\text { (Sacl+Kpnl) }\end{array}$ & This study \\
\hline
\end{tabular}




\begin{tabular}{|c|c|c|c|}
\hline Name & Vector & Insert & Reference \\
\hline pGP2972 & $\begin{array}{l}\text { pET- } \\
\text { SUMO/Bsal+Xhol }\end{array}$ & $\begin{array}{l}\text { PCR-product darB, LK80/LK81 } \\
\text { (Bsal+Xhol) }\end{array}$ & Krüger et al., 2020c \\
\hline pGP2973 & pWH844/BamHI+Sall & $\begin{array}{l}\text { PCR-product ndhF, LK201/LK202 } \\
\text { (BamHI+Sall) }\end{array}$ & This study \\
\hline pGP2974 & pUT18/Xbal+Kpnl & $\begin{array}{l}\text { PCR-product darB, LK130/LK131 } \\
\text { (Xbal+Kpnl) }\end{array}$ & Krüger et al., 2020c \\
\hline pGP2975 & pUT18c/Xbal+Kpnl & $\begin{array}{l}\text { PCR-product darB, LK130/LK131 } \\
\text { (Xbal+Kpnl) }\end{array}$ & Krüger et al., 2020c \\
\hline pGP2976 & pKT25/Xbal+Kpnl & $\begin{array}{l}\text { PCR-product darB, LK130/LK131 } \\
\text { (Xbal+Kpnl) }\end{array}$ & Krüger et al., 2020c \\
\hline pGP2977 & p25-N/Xbal+Kpnl & $\begin{array}{l}\text { PCR-product darB, LK130/LK131 } \\
\text { (Xbal+Kpnl) }\end{array}$ & Krüger et al., 2020c \\
\hline pGP2978 & pWH844/BamHI+Sall & $\begin{array}{l}\text { PCR-product ndh, LK203/LK204 } \\
\text { (BamHI+Sall) }\end{array}$ & This study \\
\hline pGP2979 & pWH844/BamHI+Sall & $\begin{array}{l}\text { PCR-product yfkC, LK205/LK206 } \\
\text { (BamHI+Sall) }\end{array}$ & This study \\
\hline pGP2980 & pGP886/BamHI+Kpnl & $\begin{array}{l}\text { PCR-product aimA, LK156/LK157 } \\
\text { (BamHI+Kpnl) }\end{array}$ & This study \\
\hline pGP2982 & pUT18/Xbal+Kpnl & $\begin{array}{l}\text { PCR-product ccpC, LK161/LK162 } \\
\text { (Xbal+Kpnl) }\end{array}$ & Krüger et al., 2020c \\
\hline pGP2983 & pUT18c/Xbal+Kpnl & $\begin{array}{l}\text { PCR-product ccpC, LK161/LK162 } \\
\text { (Xbal+Kpnl) }\end{array}$ & Krüger et al., 2020c \\
\hline pGP2984 & pKT25/Xbal+Kpnl & $\begin{array}{l}\text { PCR-product ccpC, LK161/LK162 } \\
\text { (Xbal+KpnI) }\end{array}$ & Krüger et al., 2020c \\
\hline pGP2985 & p25-N/Xbal+Kpnl & $\begin{array}{l}\text { PCR-product } c c p C \text {, LK161/LK162 } \\
\text { (Xbal+Kpnl) }\end{array}$ & Krüger et al., 2020c \\
\hline pGP2986 & $\begin{array}{l}\text { pET- } \\
\text { SUMO/Bsal+Xhol }\end{array}$ & $\begin{array}{l}\text { PCR-product aimA, LK163/LK164 } \\
\text { (Bsal+Xhol) }\end{array}$ & \\
\hline pGP2987 & pWH844/BamHI+Sall & $\begin{array}{l}\text { PCR-product aimA, LK167 /AK324 } \\
\text { (BamHI+Sall) }\end{array}$ & Krüger et al., 2020a \\
\hline pGP2988 & pWH844/BamHI+Sall & $\begin{array}{l}\text { PCR-product aimA }{ }^{N T D}, \text { LK167/LK168 } \\
\text { (BamHI+Sall) }\end{array}$ & \\
\hline pGP2989 & pGP2907/Pstl+HindIII & $\begin{array}{l}\text { PCR-product ktrD, LK169/LK170 } \\
\text { (Pstl+HindIII) }\end{array}$ & Krüger et al., 2020b \\
\hline pGP2990 & pGP1331/EcoRI+Pstl & $\begin{array}{l}\text { PCR-product } 3 \text { '-end of aimA, }^{-} \\
\text {LK171/LK172 (EcoRI+Pstl) }\end{array}$ & This study \\
\hline pGP2991 & pGP1331/EcoRI+Pstl & $\begin{array}{l}\text { PCR-product 3'-end of gltT, } \\
\text { LK173/LK174 (EcoRI+PstI) }\end{array}$ & This study \\
\hline pGP2992 & pWH844/BamHI+Sall & $\begin{array}{l}\text { PCR-product ktrB, LK175/LK176 } \\
\text { (BamHI+Sall) }\end{array}$ & This study \\
\hline pGP2993 & pWH844/BamHI+Pstl & $\begin{array}{l}\text { PCR-product kimA } \text { w520G } \\
\text { JN465/JN466 (BamHI+Pstl) }\end{array}$ & This study \\
\hline
\end{tabular}




\begin{tabular}{|c|c|c|c|}
\hline Name & Vector & Insert & Reference \\
\hline pGP2994 & pGP380/BamHI+Pstl & $\begin{array}{l}\text { PCR-product ktrC, JN459/JN460 } \\
\text { (BamHI+PstI) }\end{array}$ & This study \\
\hline pGP2995 & pGP382/BamHI+Pstl & $\begin{array}{l}\text { PCR-product ktrC, LK145/LK146 } \\
\text { (BamHI+Pstl) }\end{array}$ & This study \\
\hline pGP2996 & pWH844/Pstl+HindIII & $\begin{array}{l}\text { PCR-product ktrD, LK200/LK170 } \\
\text { (Pstl+HindIII) }\end{array}$ & This study \\
\hline pGP2997 & pWH844/BamHI+Sall & $\begin{array}{l}\text { PCR-product plsC, LK20/LK208 } \\
\text { (BamHI+Sall) }\end{array}$ & This study \\
\hline pGP2998 & $\begin{array}{l}\text { pWH844/BamHI + } \\
\text { Pstl }\end{array}$ & $\begin{array}{l}\text { PCR-product kimA } A_{\triangle 354-364} \\
\text { JN465/JN466 (BamHI+PstI) }\end{array}$ & This study \\
\hline pGP2999 & pWH844/BamHI+Pstl & $\begin{array}{l}\text { PCR-product kimA } \\
\text { (BamH112, JN465/JN466 } \\
\text { (BamH+Pstl) }\end{array}$ & This study \\
\hline pGP3000 & pGP1460/BamHI+PstI & $\begin{array}{l}\text { PCR-product gdhA (E. coli), } \\
\text { FC124/FC125, (BamHI+Pstl) }\end{array}$ & This study \\
\hline pGP3303 & pGP2907/Pstl+HindIII & $\begin{array}{l}\text { PCR-product } k \text { trD }{ }_{G 282 v} \text { LK169+LK170 } \\
\text { (Pstl+HindIII) }\end{array}$ & Krüger et al., 2020b \\
\hline pGP3305 & pWH844/Pstl+HindIII & $\begin{array}{l}\text { PCR-product } k t r D_{\text {G282v }}, \text { LK200/LK170 } \\
\text { (Pstl+HindIII) }\end{array}$ & \\
\hline pGP3306 & pBQ200/Xbal+Pstl & $\begin{array}{l}\text { PCR-product darB, LK209/TK02 } \\
\text { (Xbal+Pstl) }\end{array}$ & Krüger et al., 2020c \\
\hline pGP3308 & pGP1460/Xbal+Pstl & $\begin{array}{l}\text { PCR-product darB, LK209/TK02 } \\
\text { (Xbal+Pstl) }\end{array}$ & This study \\
\hline pGP3309 & pGP2989/BamHI+PstI & $\begin{array}{l}\text { PCR-product ktrA, JK19/JK20 } \\
\text { (BamHII+PstI) }\end{array}$ & This study \\
\hline pGP3310 & pAC5/EcoRI+BamHI & $\begin{array}{l}\text { citZ-IacZ-Fusion; LK218/LK219 } \\
\text { (EcoRI+BamHI) }\end{array}$ & This study \\
\hline pGP3312 & $\mathrm{pAC7} / \mathrm{EcoRI}+\mathrm{BamHI}$ & $\begin{array}{l}\text { pckA-lacZ-Fusion; LK226/LK227 } \\
\text { (EcoRI+BamHI) }\end{array}$ & This study \\
\hline pGP3313 & pUT18/Xbal+Kpnl & $\begin{array}{l}\text { PCR-product ccpN, LK228/LK229 } \\
\text { (Xbal+Kpnl) }\end{array}$ & This study \\
\hline pGP3314 & pUT18c/Xbal+Kpnl & $\begin{array}{l}\text { PCR-product ccpN, LK228/LK229 } \\
\text { (Xbal+Kpnl) }\end{array}$ & This study \\
\hline pGP3315 & pKT25/Xbal+Kpnl & $\begin{array}{l}\text { PCR-product ccpN, LK228/LK229 } \\
\text { (Xbal+Kpnl) }\end{array}$ & This study \\
\hline pGP3316 & p25-N/Xbal+Kpnl & $\begin{array}{l}\text { PCR-product ccpN, LK228/LK229 } \\
\text { (Xbal+Kpnl) }\end{array}$ & This study \\
\hline pGP3317 & pUT18/Xbal+Kpnl & $\begin{array}{l}\text { PCR-product guaB, LK230/LK231 } \\
\text { (Xbal+Kpnl) }\end{array}$ & This study \\
\hline pGP3318 & pUT18c/Xbal+Kpnl & $\begin{array}{l}\text { PCR-product guaB, LK230/LK231 } \\
\text { (Xbal+Kpnl) }\end{array}$ & This study \\
\hline pGP3319 & pKT25/Xbal+Kpnl & $\begin{array}{l}\text { PCR-product guaB, LK230/LK231 } \\
\text { (Xbal + Kpnl) }\end{array}$ & This study \\
\hline
\end{tabular}




\begin{tabular}{|c|c|c|c|}
\hline Name & Vector & Insert & Reference \\
\hline pGP3320 & p25-N/Xbal+Kpnl & $\begin{array}{l}\text { PCR-product guaB, LK230/LK231 } \\
\text { (Xbal+Kpnl) }\end{array}$ & This study \\
\hline pGP3321 & pUT18/Xbal+Kpnl & $\begin{array}{l}\text { PCR-product pycA, LK232/LK233 } \\
\text { (Xbal+Kpnl) }\end{array}$ & This study \\
\hline pGP3326 & pAC5/EcoRI+BamHI & $\begin{array}{l}\text { Idh-lacZ-Fusion; LK255/LK256 } \\
\text { (EcoRI+BamHI) }\end{array}$ & This study \\
\hline pGP3327 & pAC5/EcoRI+BamHI & $\begin{array}{l}\text { alsS-lacZ-Fusion; LK257/LK258 } \\
\text { (EcoRI+BamHI) }\end{array}$ & This study \\
\hline pGP3328 & $\mathrm{pAC5} / \mathrm{EcoRI}+\mathrm{BamHI}$ & $\begin{array}{l}\text { pycA-lacZ-Fusion; LK259/LK260 } \\
\text { (EcoRI+BamHI) }\end{array}$ & This study \\
\hline pGP3329 & pGP888/BamHI+Kpnl & $\begin{array}{l}\text { PCR-product rel, LK296/ LK297 } \\
\text { (BamHI+Kpnl) }\end{array}$ & This study \\
\hline pGP3330 & pWH844/BamHI+Sall & $\begin{array}{l}\text { PCR-product rel, LK283/LK284 } \\
\text { (BamHI+Sall) }\end{array}$ & This study \\
\hline pGP3331 & pGP888/Xbal+Kpnl & $\begin{array}{l}\text { PCR-product rel } \text { SYN-RRM }^{\text {LK316/LK297 }} \\
\text { (Xbal+Kpnl) }\end{array}$ & This study \\
\hline pGP3332 & $\begin{array}{l}\text { pET- } \\
\text { SUMO/Bsal+Xhol }\end{array}$ & $\begin{array}{l}\text { PCR-product darB }{ }^{\text {Y45S }} \\
\text { LK80/LK81/LK261 (Bsal+Xhol) }\end{array}$ & This study \\
\hline pGP3333 & pBQ200/Xbal+Pstl & $\begin{array}{l}\text { PCR-product darB }{ }^{\text {Y45S }} \\
\text { LK209/TK02/LK261 (Xbal+Pstl) }\end{array}$ & This study \\
\hline pGP3334 & pGP888/BamHI+Kpnl & $\begin{array}{l}\text { PCR-product rel } \\
\text { (BTD, , LK296/LK318 } \\
\text { Bl+Kpnl) }\end{array}$ & This study \\
\hline pGP3435 & pWH844/BamHI+Sall & $\begin{array}{l}\text { PCR-product rel } \\
\text { LK28, D264G, } \\
\text { LK28K358 (BamHI+Sall) }\end{array}$ & This study \\
\hline pGP3336 & pUT18/Xbal+Kpnl & $\begin{array}{l}\text { PCR-product sasA, LK312/LK313 } \\
\text { (Xbal+Kpnl) }\end{array}$ & Krüger et al., 2020c \\
\hline pGP3337 & pUT18c/Xbal+Kpnl & $\begin{array}{l}\text { PCR-product sasA, LK312/LK313 } \\
\text { (Xbal+Kpnl) }\end{array}$ & Krüger et al., 2020c \\
\hline pGP3338 & pKT25/Xbal+Kpnl & $\begin{array}{l}\text { PCR-product sasA, LK312/LK313 } \\
\text { (Xbal+Kpnl) }\end{array}$ & Krüger et al., 2020c \\
\hline pGP3339 & p25-N/Xbal+Kpnl & $\begin{array}{l}\text { PCR-product sasA, LK312/LK313 } \\
\text { (Xbal+Kpnl) }\end{array}$ & Krüger et al., 2020c \\
\hline pGP3340 & pUT18/Xbal+Kpnl & $\begin{array}{l}\text { PCR-product ktrC, LK285/LK286 } \\
\text { (Xbal+Kpnl) }\end{array}$ & This study \\
\hline pGP3341 & pUT18c/Xbal+Kpnl & $\begin{array}{l}\text { PCR-product ktrC, LK285/LK286 } \\
\text { (Xbal+Kpnl) }\end{array}$ & This study \\
\hline pGP3342 & pKT25/Xbal+Kpnl & $\begin{array}{l}\text { PCR-product ktrC, LK285/LK286 } \\
\text { (Xbal+Kpnl) }\end{array}$ & This study \\
\hline pGP3343 & p25-N/Xbal+Kpnl & $\begin{array}{l}\text { PCR-product ktrC, LK285/LK286 } \\
\text { (Xbal+Kpnl) }\end{array}$ & This study \\
\hline pGP3344 & pUT18/Xbal+Kpnl & $\begin{array}{l}\text { PCR-product rel, LK287/LK288 } \\
\text { (Xbal+Kpnl) }\end{array}$ & Krüger et al., 2020c \\
\hline
\end{tabular}




\begin{tabular}{|c|c|c|c|}
\hline Name & Vector & Insert & Reference \\
\hline pGP3345 & pUT18c/Xbal+Kpnl & $\begin{array}{l}\text { PCR-product rel, LK287/LK288 } \\
\text { (Xbal+Kpnl) }\end{array}$ & Krüger et al., 2020c \\
\hline pGP3346 & pKT25/Xbal+Kpnl & $\begin{array}{l}\text { PCR-product rel, LK287/LK288 } \\
\text { (Xbal+Kpnl) }\end{array}$ & Krüger et al., 2020c \\
\hline pGP3347 & p25-N/Xbal+Kpnl & $\begin{array}{l}\text { PCR-product rel, LK287/LK288 } \\
\text { (Xbal+Kpnl) }\end{array}$ & Krüger et al., 2020c \\
\hline pGP3348 & pGP172/Kpnl+BamHI & $\begin{array}{l}\text { PCR-product rel LK310/LK311 } \\
\text { (Xbal+KpnI) }\end{array}$ & Krüger et al., 2020c \\
\hline pGP3350 & pGP172/Kpnl+BamHI & $\begin{array}{l}\text { PCR-product rel } \text { IND }^{\text {LK310/LK333 }} \\
\text { (Kpnl+BamHI) }\end{array}$ & Krüger et al., 2020c \\
\hline pGP3411 & pUT18/Xbal+Kpnl & $\begin{array}{l}\text { PCR-product sasB, LK314/LK315 } \\
\text { (Xbal+Kpnl) }\end{array}$ & Krüger et al., 2020c \\
\hline pGP3412 & pUT18c/Xbal+Kpnl & $\begin{array}{l}\text { PCR-product sasB, LK314/LK315 } \\
\text { (Xbal+Kpnl) }\end{array}$ & Krüger et al., 2020c \\
\hline pGP3413 & pKT25/Xbal+Kpnl & $\begin{array}{l}\text { PCR-product sasB, LK314/LK315 } \\
\text { (Xbal+Kpnl) }\end{array}$ & Krüger et al., 2020c \\
\hline pGP3414 & p25-N/Xbal+Kpnl & $\begin{array}{l}\text { PCR-product sasB, LK314/LK315 } \\
\text { (Xbal+Kpnl) }\end{array}$ & Krüger et al., 2020c \\
\hline pGP3415 & pUT18/Xbal+Kpnl & $\begin{array}{l}\text { PCR-product rel/SYN-RRM, LK316/LK288 } \\
\text { (Xbal+Kpnl) }\end{array}$ & Krüger et al., 2020c \\
\hline pGP3416 & pUT18c/Xbal+Kpnl & $\begin{array}{l}\text { PCR-product re/ IYN-RRM, LK316/LK288 } \\
\text { (Xbal+Kpnl) }\end{array}$ & Krüger et al., 2020c \\
\hline pGP3417 & pKT25/Xbal+Kpnl & $\begin{array}{l}\text { PCR-product rel } \text { IYN-RRM }^{\mathrm{YN}} \text { LK16/LK288 } \\
\text { (Xbal+Kpnl) }\end{array}$ & Krüger et al., 2020c \\
\hline pGP3418 & p25-N/Xbal+Kpnl & 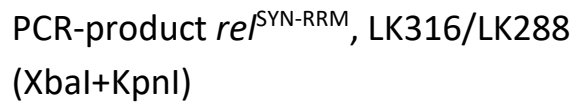 & Krüger et al., 2020c \\
\hline pGP3419 & pUT18/Xbal+Kpnl & $\begin{array}{l}\text { PCR-product rel NTD, LK287/LK317 } \\
\text { (Xbal+Kpnl) }\end{array}$ & Krüger et al., 2020c \\
\hline pGP3420 & pUT18c/Xbal+Kpnl & $\begin{array}{l}\text { PCR-product rel NTD, LK287/LK317 } \\
\text { (Xbal+Kpnl) }\end{array}$ & Krüger et al., 2020c \\
\hline pGP3421 & pKT25/Xbal+Kpnl & $\begin{array}{l}\text { PCR-product rel NTD, LK287/LK317 } \\
\text { (Xbal+Kpnl) }\end{array}$ & Krüger et al., 2020c \\
\hline pGP3422 & p25-N/Xbal+Kpnl & $\begin{array}{l}\text { PCR-product rel NTD, LK287/LK317 } \\
\text { (Xbal+Kpnl) }\end{array}$ & Krüger et al., 2020c \\
\hline pGP3429 & pWH844/BamHI+Sall & $\begin{array}{l}\text { PCR-product rel NTD, LK283/LK358 } \\
\text { (BamHI+Sall) }\end{array}$ & Krüger et al., 2020c \\
\hline pGP3430 & pGP380/BamHI+Sall & $\begin{array}{l}\text { PCR-product rel, LK283/LK284 } \\
\text { (BamHI+Sall) }\end{array}$ & This study \\
\hline pGP3431 & pGP382/BamHI+Sall & $\begin{array}{l}\text { PCR-product rel, LK369/LK370 } \\
\text { (BamHI+Sall) }\end{array}$ & This study \\
\hline pGP3432 & pGP172/Kpnl+BamHI & $\begin{array}{l}\text { PCR-product rel } \text { D264G }^{\text {LK310/LK311 }} \\
\text { (Kpnl+BamHI) }\end{array}$ & This study \\
\hline
\end{tabular}




\begin{tabular}{|c|c|c|c|}
\hline Name & Vector & Insert & Reference \\
\hline pGP3433 & pGP172/KpnI+BamHI & $\begin{array}{l}\text { PCR-product rel/ND LK310/LK333 } \\
\text { (Kpnl+BamHI) }\end{array}$ & This study \\
\hline pGP3434 & pWH844/BamHI+Sall & $\begin{array}{l}\text { PCR-product rel } \text { D264G }^{\text {LK283/LK284 }} \\
\text { (BamHI+Sall) }\end{array}$ & This study \\
\hline pGP3435 & pWH844/BamHI+Sall & $\begin{array}{l}\text { PCR-product rel }{ }^{N T D, D 264 G}, \\
\text { LK283/LK358 (BamHI+Sall) }\end{array}$ & This study \\
\hline pGP3436 & pBQ200/Xbal+Pstl & $\begin{array}{l}\text { PCR-product darB }{ }^{\text {K23E, }} \\
\text { LK209/TK02/LK371 (Xbal+Pstl) }\end{array}$ & This study \\
\hline pGP3437 & pBQ200/Xbal+Pstl & $\begin{array}{l}\text { PCR-product darB }{ }^{\text {A25G }} \\
\text { LK209/TK02/LK372 (Xbal+Pstl) }\end{array}$ & Krüger et al., 2020c \\
\hline pGP3438 & pBQ200/Xbal+Pstl & $\begin{array}{l}\text { PCR-product darB }{ }^{\text {T46V }} \\
\text { LK209/TK02/LK373 (Xbal+Pstl) }\end{array}$ & This study \\
\hline pGP3439 & pBQ200/Xbal+Pstl & $\begin{array}{l}\text { PCR-product darB }{ }^{\text {A47G, }} \\
\text { LK209/TK02/LK374 (Xbal+Pstl) }\end{array}$ & This study \\
\hline pGP3440 & pBQ200/Xbal+Pstl & $\begin{array}{l}\text { PCR-product darB }{ }^{\text {F115A }} \\
\text { LK209/TK02/LK375 (Xbal+Pstl) }\end{array}$ & This study \\
\hline pGP3441 & pBQ200/Xbal+Pstl & $\begin{array}{l}\text { PCR-product darB }{ }^{\text {R132M }}, \\
\text { LK209/TK02/LK376 (Xbal+Pstl) }\end{array}$ & Krüger et al., 2020c \\
\hline pGP3442 & pBQ200/Xbal+Pstl & $\begin{array}{l}\text { PCR-product darB }{ }^{\text {R131L }} \\
\text { LK209/TK02/LK376 (Xbal+Pstl) }\end{array}$ & This study \\
\hline pGP3443 & $\begin{array}{l}\text { pET- } \\
\text { SUMO/Xhol+Bsal }\end{array}$ & $\begin{array}{l}\text { PCR-product darB }{ }^{\mathrm{K} 23 \mathrm{E}}, \mathrm{LK} 80 / \mathrm{LK} 81 \\
\text { (Xhol+Bsal) }\end{array}$ & This study \\
\hline pGP3444 & $\begin{array}{l}\text { pET- } \\
\text { SUMO/Xhol+Bsal }\end{array}$ & $\begin{array}{l}\text { PCR-product darB }{ }^{\mathrm{A} 25 \mathrm{G}}, \mathrm{LK} 80 / \mathrm{LK} 81 \\
\text { (Xhol+Bsal) }\end{array}$ & Krüger et al. 2020c \\
\hline pGP3445 & $\begin{array}{l}\text { pET- } \\
\text { SUMO/Xhol+Bsal }\end{array}$ & $\begin{array}{l}\text { PCR-product darB }{ }^{\text {T46V }}, \text { LK80/LK81 } \\
\text { (Xhol+Bsal) }\end{array}$ & This study \\
\hline pGP3446 & $\begin{array}{l}\text { pET- } \\
\text { SUMO/Xhol+Bsal }\end{array}$ & $\begin{array}{l}\text { PCR-product darB }{ }^{\mathrm{A} 47 \mathrm{G}}, \mathrm{LK} 80 / \mathrm{LK} 81 \\
\text { (Xhol+Bsal) }\end{array}$ & This study \\
\hline pGP3447 & $\begin{array}{l}\text { pET- } \\
\text { SUMO/Xhol+Bsal }\end{array}$ & $\begin{array}{l}\text { PCR-product darB }{ }^{\mathrm{F115A}}, \mathrm{LK} 80 / \mathrm{LK} 81 \\
\text { (Xhol+Bsal) }\end{array}$ & This study \\
\hline pGP3448 & $\begin{array}{l}\text { pET- } \\
\text { SUMO/Xhol+Bsal }\end{array}$ & $\begin{array}{l}\text { PCR-product darB }{ }^{\text {R132M }}, \text { LK80/LK81 } \\
\text { (Xhol+Bsal) }\end{array}$ & Krüger et al., 2020c \\
\hline pGP3449 & $\begin{array}{l}\text { pET- } \\
\text { SUMO/Xhol+Bsal }\end{array}$ & $\begin{array}{l}\text { PCR-product darB }{ }^{\text {R131L }}, \text { LK80/LK81 } \\
\text { (Xhol+Bsal) }\end{array}$ & This study \\
\hline pGP3456 & pGP380/BamHI+Sall & $\begin{array}{l}\text { PCR-product sasA, LK383 + LK384 } \\
\text { (BamHI+Sall) }\end{array}$ & This study \\
\hline pGP3457 & pGP382/BamHI+Sall & $\begin{array}{l}\text { PCR-product sasA, LK381 + LK382 } \\
\text { (BamHI+Sall) }\end{array}$ & This study \\
\hline pGP3459 & $\begin{array}{l}\text { pET- } \\
\text { SUMO/Bsal+Xhol }\end{array}$ & $\begin{array}{l}\text { PCR-product rel LK387/LK388 } \\
\text { (Bsal+Xhol) }\end{array}$ & This study \\
\hline pGP3460 & $\begin{array}{l}\text { pET- } \\
\text { SUMO/Bsal+Xhol }\end{array}$ & $\begin{array}{l}\text { PCR-product darB }{ }^{\mathrm{A} 25 \mathrm{G} / \mathrm{R} 132 \mathrm{M}}, \\
\text { LK80/LK81 (Bsal+Xhol) }\end{array}$ & Krüger et al., 2020c \\
\hline
\end{tabular}




\begin{tabular}{|c|c|c|c|}
\hline Name & Vector & Insert & Reference \\
\hline \multirow[t]{2}{*}{ pGP3461 } & pET- & PCR-product darB ${ }^{\mathrm{A25G/R131L}}$ & This study \\
\hline & SUMO/Bsal+Xhol & LK80/LK81 (Xhol+Bsal) & \\
\hline \multirow[t]{2}{*}{ pGP3462 } & pET- & PCR-product darB ${ }^{\text {Y45S/R131L LK80/LK81 }}$ & This study \\
\hline & SUMO/Bsal+Xhol & (Xhol+Bsal) & \\
\hline \multirow[t]{2}{*}{ pGP3463 } & pWH844/BamHI+Sall & PCR-product $m p f A^{214-349 a a}$ & This study \\
\hline & & LK394/LK395 (BamHI+Sall) & \\
\hline \multirow[t]{2}{*}{ pGP3464 } & pWH844/BamHI+Sall & PCR-product $y h d T^{215-351 a a}$, & This study \\
\hline & & LK396/LK397 (BamHI+SalI) & \\
\hline \multirow[t]{2}{*}{ pGP3465 } & pWH844/BamHI+Sall & PCR-product $y r k A^{214-350 a a}$ & This study \\
\hline & & LK398/LK399 (BamHI+Sall) & \\
\hline \multirow[t]{2}{*}{ pGP3466 } & pWH844/BamHI+Sall & 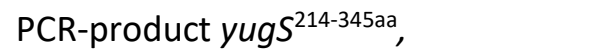 & This study \\
\hline & & LK400/LK401 (BamHI+Sall) & \\
\hline \multirow[t]{2}{*}{ pGP3467 } & pWH844/BamHI+Sall & PCR-product yqhB ${ }^{223-359 a a}$ & This study \\
\hline & & LK402/LK403 (BamHI+Sall) & \\
\hline \multirow[t]{2}{*}{ pGP3468 } & pET- & PCR-Prod. $m p f A^{214-349 a a}$, LK404/LK405 & This study \\
\hline & SUMO/Bsal+Xhol & (Xhol+Bsal) & \\
\hline \multirow[t]{2}{*}{ pGP3469 } & pET- & PCR-product $y h d T^{215-351 a a}$ & This study \\
\hline & SUMO/Bsal+Xhol & LK406/LK407 (Xhol+Bsal) & \\
\hline \multirow[t]{2}{*}{ pGP3470 } & pET- & PCR-product $y r k A^{214-350 a a}$, & This study \\
\hline & SUMO/Bsal+Xhol & LK408/LK409 (Xhol+Bsal) & \\
\hline \multirow[t]{2}{*}{ pGP3471 } & pET- & PCR-product yugS $S^{214-345 a a,}$ & This study \\
\hline & SUMO/Bsal+Xhol & LK410/LK411 (Xhol+Bsal) & \\
\hline \multirow[t]{2}{*}{ pGP3472 } & pET- & PCR-product yqhB $B^{223-359 a a,}$ & This study \\
\hline & SUMO/Bsal+Xhol & LK412/LK413 (Xhol+Bsal) & \\
\hline \multirow[t]{2}{*}{ pGP3474 } & pET- & PCR-product darB $B^{\text {D93R }}$ & This study \\
\hline & SUMO/Xhol+Bsal & LK80/LK81/LK414 (Xhol+Bsal) & \\
\hline \multirow[t]{2}{*}{ pGP3475 } & pET- & PCR-product darB ${ }^{\mathrm{N} 68 \mathrm{R}}$ & This study \\
\hline & SuMO/Xhol+Bsal & LK80/LK81/LK415 (Xhol+Bsal) & \\
\hline \multirow[t]{2}{*}{ pGP3476 } & pET- & PCR-product darB ${ }^{\mathrm{T} 92 \mathrm{~W}}$ & This study \\
\hline & SUMO/Xhol+Bsal & LK80/LK81/LK416 (Xhol+Bsal) & \\
\hline \multirow[t]{2}{*}{ pGP3477 } & pET- & PCR-product darB $B^{\mathrm{N} 68 \mathrm{Y}}$ & This study \\
\hline & SUMO/Xhol+Bsal & LK80/LK81/LK417 (Xhol+Bsal) & \\
\hline \multirow[t]{2}{*}{ pGP3478 } & pET- & PCR-product darB ${ }^{\mathrm{R} 132 \mathrm{E}}$ & This study \\
\hline & SUMO/Xhol+Bsal & LK80/LK81/LK418 (Xhol+Bsal) & \\
\hline \multirow[t]{2}{*}{ pGP3479 } & pET- & PCR-product darB ${ }^{\mathrm{K} 136 \mathrm{E}}$ & This study \\
\hline & SUMO/Xhol+Bsal & LK80/LK81/LK419 (Xhol+Bsal) & \\
\hline \multirow[t]{2}{*}{ pGP3480 } & pET- & PCR-product darB ${ }^{\mathrm{K} 42 \mathrm{Y}}$ & This study \\
\hline & SUMO/Xhol+Bsal & LK80/LK81/LK420 (Xhol+Bsal) & \\
\hline \multirow[t]{2}{*}{ pGP3481 } & pET- & PCR-product darB $B^{\mathrm{L38F}}$ & This study \\
\hline & SUMO/Xhol+Bsal & LK80/LK81/LK421 (Xhol+Bsal) & \\
\hline \multirow[t]{2}{*}{ pGP3483 } & pET- & PCR-product darB ${ }^{\text {N68R/D93R }}$ & This study \\
\hline & SuMO/Xhol+Bsal & LK80/LK81/LK414 (Xhol+Bsal) & \\
\hline
\end{tabular}




\begin{tabular}{llll}
\hline Name & Vector & Insert & Reference \\
\hline pGP3484 & pET- & PCR-product $\operatorname{dar} B^{\mathrm{N} 68 Y / T 92 W}$, & This study \\
& SUMO/Xhol+Bsal & LK80/LK81/LK416 (Xhol+Bsal) & \\
pGP3486 & pET- & PCR-product darB ${ }^{\text {K42Y/N68Y }}$, & This study \\
& SUMO/Xhol+Bsal & LK80/LK81/LK420 (Xhol+Bsal) & \\
\hline
\end{tabular}


